University of Tennessee Health Science Center

UTHSC Digital Commons

$12-2013$

\title{
Signal Transduction and Transcriptional Regulation Pathways Essential for Azole Resistance in Candida albicans
}

Erin M. Vasicek

University of Tennessee Health Science Center

Follow this and additional works at: https://dc.uthsc.edu/dissertations

Part of the Bacterial Infections and Mycoses Commons, and the Fungi Commons

\section{Recommended Citation}

Vasicek, Erin M. , "Signal Transduction and Transcriptional Regulation Pathways Essential for Azole Resistance in Candida albicans" (2013). Theses and Dissertations (ETD). Paper 288. http://dx.doi.org/ 10.21007/etd.cghs.2013.0335.

This Dissertation is brought to you for free and open access by the College of Graduate Health Sciences at UTHSC Digital Commons. It has been accepted for inclusion in Theses and Dissertations (ETD) by an authorized administrator of UTHSC Digital Commons. For more information, please contact jwelch30@uthsc.edu. 


\title{
Signal Transduction and Transcriptional Regulation Pathways Essential for Azole Resistance in Candida albicans
}

\author{
Abstract \\ Candida albicans is the most prevalent human fungal pathogen, found as a commensal organism in the \\ mucosa, gastrointestinal, and urogenital tracts of humans. This pathogenic fungus causes a wide \\ spectrum of diseases, including the mucosal infection oropharyngeal candidiasis (OPC) which frequently \\ effects patients with human immunodeficiency virus (HIV). The azole antifungals (such as fluconazole) \\ are the most widely used and important ergosterol biosynthesis inhibitors (EBIs) for the treatment of \\ Candida infections, including OPC. However, the azoles are fungistatic against $\mathrm{C}$. albicans and therefore \\ have limited efficacy against this organism, especially for immunocompromised patients. In C. albicans, \\ the transcription factor Upc2 is central to the regulation of ergosterol biosynthesis. UPC2 activating \\ mutations contribute to azole resistance, whereas disruption increases azole susceptibility. We further \\ investigated the relationship of UPC2 to fluconazole susceptibility, particularly in azole-resistant strains. \\ In addition to the reduced fluconazole minimum inhibitory concentration (MIC) previously observed with \\ UPC2 disruption, we observed a reduced minimum fungicidal concentration (MFC) in a upc $2 \Delta / \Delta$ mutant \\ relative to its azole-susceptible parent SC5314. Moreover, upc $2 \Delta / \Delta$ was unable to grow on solid media \\ containing $10 \mu \mathrm{g} / \mathrm{mL}$ fluconazole and exhibited increased susceptibility and a clear zone of inhibition \\ when subjected to Etest. Time-kill analysis showed increased azole activity against upc $2 \Delta / \Delta$ compared to \\ SC5314. UPC2 disruption in strains carrying specific resistance mutations also resulted in reduced MICs \\ and MFCs. UPC2 disruption in a highly azole-resistant clinical isolate containing multiple resistance \\ mechanisms likewise resulted in a reduced MIC and MFC. This mutant was unable to grow on solid media \\ containing $10 \mu \mathrm{g} / \mathrm{mL}$ fluconazole and exhibited increased susceptibility and a clear zone of inhibition \\ when subjected to Etest. Time-kill analysis showed increased azole activity against the upc $2 \Delta / \Delta$ mutant \\ in the resistant background. Microarray analysis showed attenuated fluconazole induction of genes \\ involved in sterol biosynthesis as well as iron transport and homeostasis in the absence of UPC2. Taken \\ together, our results demonstrate that the UPC2 transcriptional network is universally essential for azole \\ resistance in $\mathrm{C}$. albicans and represents an attractive target for enhancing azole antifungal activity. \\ Fungal survival in the presence of the azoles is permitted by specific signal transduction and \\ transcriptional activation programs. In an effort to identify additional transcriptional pathways involved in \\ fluconazole susceptibility, we sought to identify transcription factors (TFs) essential for this process. \\ From a collection of C. albicans strains disrupted for genes encoding TFs (Homann et al., PLoS Genet. \\ 2009;5:e1000783), 4 exhibited marked reduction in MFC in both RPMI and YPD media independent of any \\ noted growth defect in medium alone as compared to the parent strain. In addition to UPC2, one gene of \\ interest (GOI) (CAS5) was unable to recover from fluconazole exposure at concentrations as low as $2 \mu \mathrm{g} /$ \\ $\mathrm{mL}$ after 72 hours in YPD medium, showed reduced susceptibility and a clear zone of inhibition by Etest, \\ was unable to grow on solid media containing $10 \mu \mathrm{g} / \mathrm{mL}$ fluconazole, and exhibited increased \\ susceptibility by time-kill analysis. CAS5 disruption in highly azole resistant clinical isolates containing \\ multiple resistance mechanisms did not alter susceptibility. However, CAS5 disruption in strains \\ containing specific resistance mutations resulted in a moderate reduction in MIC and MFC. Genome-wide \\ transcriptional analysis was performed in the presence of fluconazole and was consistent with the \\ suggested role of CAS5 in cell wall organization while also suggesting a role in iron transport and \\ homeostasis. These findings suggest that Cas 5 regulates a transcriptional network that influences \\ susceptibility of $\mathrm{C}$. albicans to fluconazole. Further delineation of this transcriptional network may identify \\ targets for potential cotherapeutic strategies to enhance the activity to the azole class of antifungals. \\ Genome-wide expression profiling identified that ergosterol biosynthesis genes depend on Upc2 for their \\ fluconazole-induceability, and cell wall maintenance genes depend on Cas 5 . In the presence of \\ fluconazole, the expression of iron ion transport and homeostasis genes were also identified to be \\ dependent on Upc2 as well as Cas5. The identification of these target genes validated the known and
}


putative roles for Upc2 and Cas5, and also provided insight into novel roles for these transcription factors in azole susceptibility.

\section{Document Type}

Dissertation

Degree Name

Doctor of Philosophy $(\mathrm{PhD})$

\section{Program}

Biomedical Sciences

\section{Research Advisor}

P. David Rogers, Pharm.D, Ph.D.

\section{Keywords}

Antifungal, azole, Candida albicans, Rrsistance, transcriptional regulation

\section{Subject Categories}

Bacterial Infections and Mycoses | Diseases | Fungi | Medicine and Health Sciences | Organisms 
Signal Transduction and Transcriptional Regulation Pathways Essential for Azole Resistance in Candida albicans

\author{
A Dissertation \\ Presented for \\ The Graduate Studies Council \\ The University of Tennessee \\ Health Science Center \\ In Partial Fulfillment \\ Of the Requirements for the Degree \\ Doctor of Philosophy \\ From The University of Tennessee
}

By

Erin M. Vasicek

December 2013 
Copyright (C) 2013 by Erin M. Vasicek. All rights reserved. 


\section{DEDICATION}

To my family and friends for their love, patience, and encouragement. No amount of homemade cookies can express my gratitude. 


\section{ACKNOWLEDGEMENTS}

First I would like to express my utmost gratitude to my advisor, Dave Rogers, who challenged me throughout my research and provided opportunities to interact with other researchers while experiencing the country, and the world. I would also like to thank the members of my committee, Jim Dale, Ramin Homayouni, Richard Lee, and Nathan Wiederhold for their suggestions, guidance, and critical reviews in order to improve my research.

My gratitude also extends to the current and former members of the Rogers lab, Kathy Barker, Kelly Caudle, Chris Hoehamer, Teresa Liu, Stephanie Flowers, Sarah Whaley, Beth Berkow, Qing Zhang, Andy Nishimoto, and Gabaergic Kayihura for their friendship and support. I am also extremely grateful to Todd Reynolds at University of Tennessee in Knoxville, Tennessee for allowing me to spend a month working in his lab.

Last but not least, I would like to thank my entire family for believing in me throughout this adventure. In addition, I would like to thank all my friends, near and far, for keeping me balanced. 


\begin{abstract}
Candida albicans is the most prevalent human fungal pathogen, found as a commensal organism in the mucosa, gastrointestinal, and urogenital tracts of humans. This pathogenic fungus causes a wide spectrum of diseases, including the mucosal infection oropharyngeal candidiasis (OPC) which frequently effects patients with human immunodeficiency virus (HIV). The azole antifungals (such as fluconazole) are the most widely used and important ergosterol biosynthesis inhibitors (EBIs) for the treatment of Candida infections, including OPC. However, the azoles are fungistatic against $C$. albicans and therefore have limited efficacy against this organism, especially for immunocompromised patients.
\end{abstract}

In C. albicans, the transcription factor Upc 2 is central to the regulation of ergosterol biosynthesis. UPC2 activating mutations contribute to azole resistance, whereas disruption increases azole susceptibility. We further investigated the relationship of $U P C 2$ to fluconazole susceptibility, particularly in azole-resistant strains. In addition to the reduced fluconazole minimum inhibitory concentration (MIC) previously observed with UPC2 disruption, we observed a reduced minimum fungicidal concentration (MFC) in a upc $2 \Delta / \Delta$ mutant relative to its azole-susceptible parent SC5314. Moreover, upc $2 \Delta / \Delta$ was unable to grow on solid media containing $10 \mu \mathrm{g} / \mathrm{mL}$ fluconazole and exhibited increased susceptibility and a clear zone of inhibition when subjected to Etest. Time-kill analysis showed increased azole activity against upc $2 \Delta / \Delta$ compared to SC5314. UPC2 disruption in strains carrying specific resistance mutations also resulted in reduced MICs and MFCs. UPC2 disruption in a highly azole-resistant clinical isolate containing multiple resistance mechanisms likewise resulted in a reduced MIC and MFC. This mutant was unable to grow on solid media containing $10 \mu \mathrm{g} / \mathrm{mL}$ fluconazole and exhibited increased susceptibility and a clear zone of inhibition when subjected to Etest. Time-kill analysis showed increased azole activity against the upc $2 \Delta / \Delta$ mutant in the resistant background. Microarray analysis showed attenuated fluconazole induction of genes involved in sterol biosynthesis as well as iron transport and homeostasis in the absence of $U P C 2$. Taken together, our results demonstrate that the UPC2 transcriptional network is universally essential for azole resistance in $C$. albicans and represents an attractive target for enhancing azole antifungal activity.

Fungal survival in the presence of the azoles is permitted by specific signal transduction and transcriptional activation programs. In an effort to identify additional transcriptional pathways involved in fluconazole susceptibility, we sought to identify transcription factors (TFs) essential for this process. From a collection of C. albicans strains disrupted for genes encoding TFs (Homann et al., PLoS Genet. 2009;5:e1000783), 4 exhibited marked reduction in MFC in both RPMI and YPD media independent of any noted growth defect in medium alone as compared to the parent strain. In addition to $U P C 2$, one gene of interest (GOI) $(C A S 5)$ was unable to recover from fluconazole exposure at concentrations as low as $2 \mu \mathrm{g} / \mathrm{mL}$ after 72 hours in YPD medium, showed reduced susceptibility and a clear zone of inhibition by Etest, was unable to grow on solid media containing $10 \mu \mathrm{g} / \mathrm{mL}$ fluconazole, and exhibited increased susceptibility by time- 
kill analysis. CAS5 disruption in highly azole resistant clinical isolates containing multiple resistance mechanisms did not alter susceptibility. However, CAS5 disruption in strains containing specific resistance mutations resulted in a moderate reduction in MIC and MFC. Genome-wide transcriptional analysis was performed in the presence of fluconazole and was consistent with the suggested role of $C A S 5$ in cell wall organization while also suggesting a role in iron transport and homeostasis. These findings suggest that Cas5 regulates a transcriptional network that influences susceptibility of C. albicans to fluconazole. Further delineation of this transcriptional network may identify targets for potential co-therapeutic strategies to enhance the activity to the azole class of antifungals.

Genome-wide expression profiling identified that ergosterol biosynthesis genes depend on Upc2 for their fluconazole-induceability, and cell wall maintenance genes depend on Cas5. In the presence of fluconazole, the expression of iron ion transport and homeostasis genes were also identified to be dependent on Upc2 as well as Cas5. The identification of these target genes validated the known and putative roles for Upc2 and Cas5, and also provided insight into novel roles for these transcription factors in azole susceptibility. 


\section{TABLE OF CONTENTS}

CHAPTER 1. INTRODUCTION .................................................................................

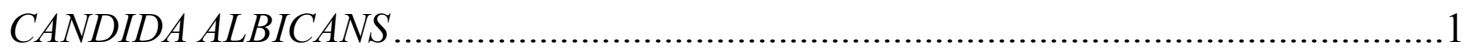

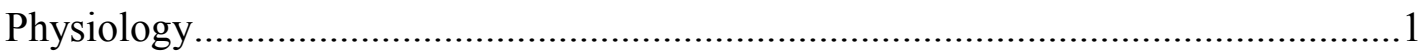

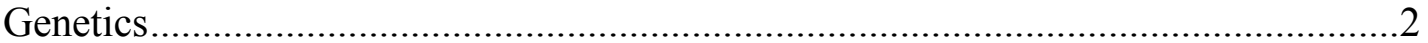

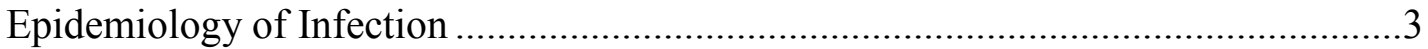

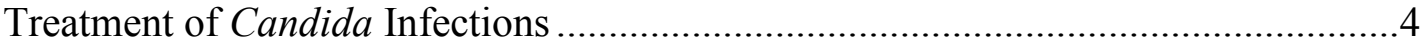

FUNGISTATIC AZOLE ACTIVITY AND AZOLE RESISTANCE ..........................5

Clinical Impact of Fungistatic Azole Activity ..........................................................

Response Mechanisms That Allow Fungistatic Azole Activity ...............................6

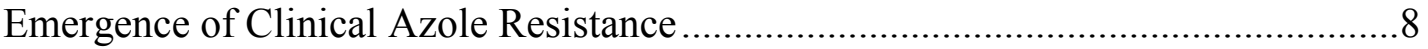

Molecular Mechanisms of Resistance to Azoles ....................................................9

TRANSCRIPTIONAL REGULATORS .............................................................. 11

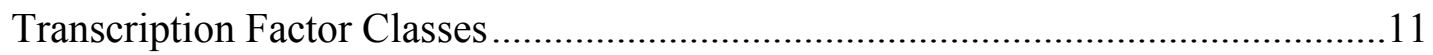

Transcription Factors and Fungistatic Azole Activity ...........................................12

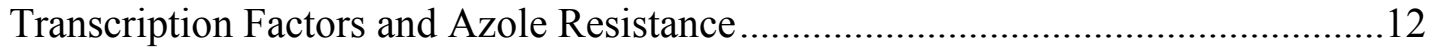

CENTRAL HYPOTHESIS AND RESEARCH OBJECTIVES ................................14

\section{CHAPTER 2. UPC2 IS UNIVERSALLY ESSENTIAL FOR AZOLE RESISTANCE IN CANDIDA ALBICANS ...........................................................16}

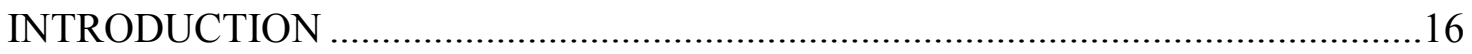

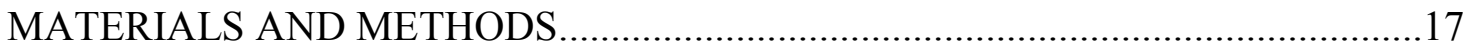

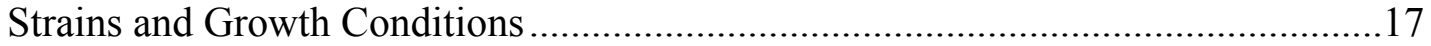

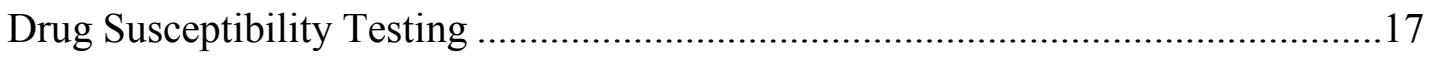

Construction of ERG11 Strains with Mutant Alleles................................................17

Construction of UPC2 Deletion Strains ................................................................19

Isolation of Genomic DNA and Southern Hybridization.......................................19

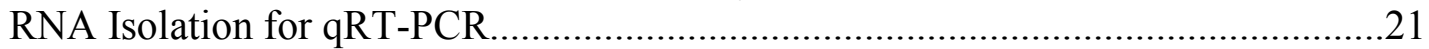

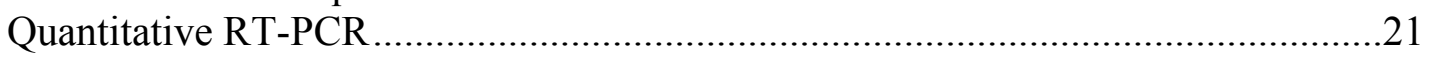

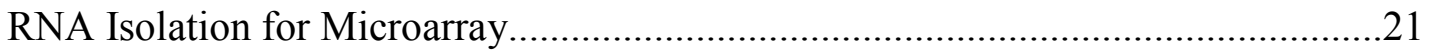

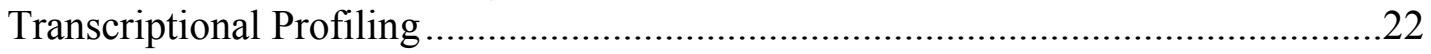

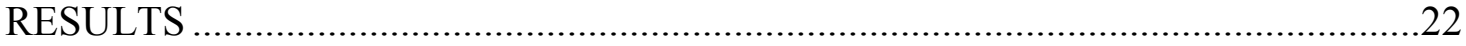

Disruption of UPC2 Results in Enhanced Fluconazole Activity.............................22

Disruption of UPC2 in Strains Containing Resistance Mutations in MRR1,

$T A C 1$, and ERG11 Also Enhances Fluconazole Activity .....................................26

Disruption of Upc2 Overrides Clinical Drug Resistance........................................26

Expression of ERG11,CDR1,CDR2, and MDR1 When UPC2 Is Disrupted in

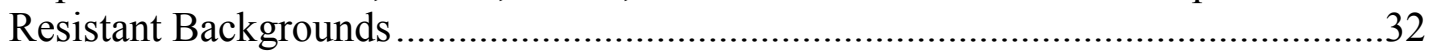

Comparison of the Gene Expression Profiles of Wild-Type Strain SC5314 and

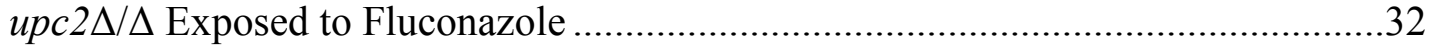

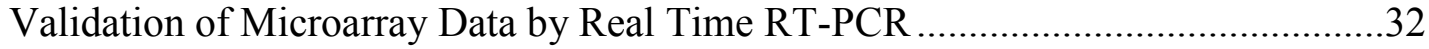

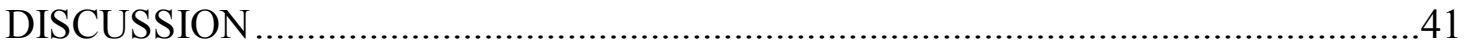




\section{CHAPTER 3. CAS5 IMPACTS THE ACTIVITY OF FLUCONAZOLE AGAINST CANDIDA ALBICANS}

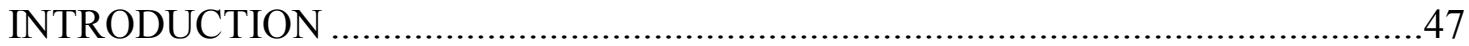

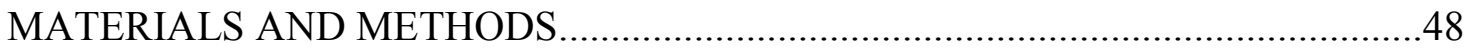

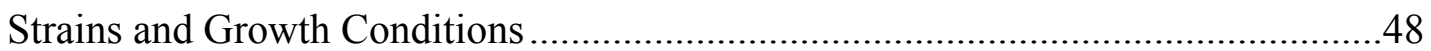

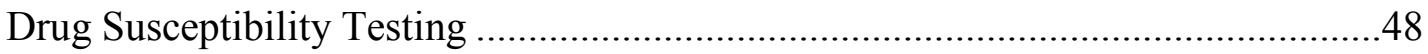

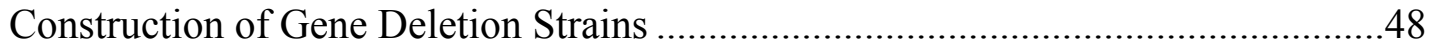

Isolation of Genomic DNA and Southern Hybridization......................................52

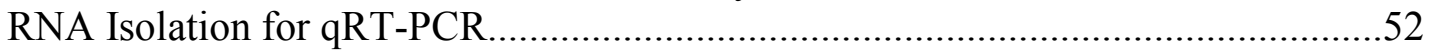

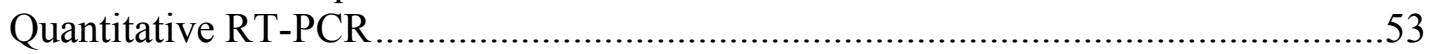

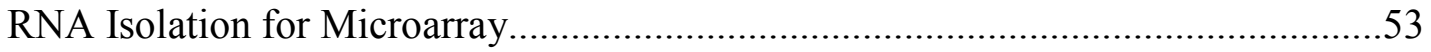

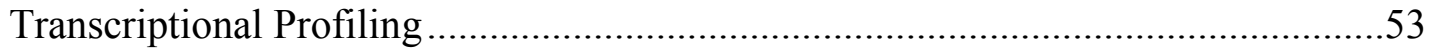

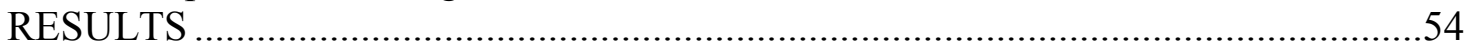

Disruption of Cas5 Enhances Fluconazole Activity against C. albicans....................54

$M K C 1$ Disruption Does Not Fully Phenocopy CAS5 ..........................................59

Disruption of Cas5 Does Not Override Combinations of Clinical Drug

Resistance Mechanisms

Disruption of $C A S 5$ in Strains Containing Resistance Mutations in MRR1, TAC1, and ERG11 Impacts Fluconazole Susceptibility .....................................................63

Expression of ERG11,CDR1,CDR2, and MDR1 When CAS5 Is Disrupted in

Resistant Backgrounds

Comparison of the Gene Expression Profiles of Wild-Type Strain SC5314 and cas $5 \Delta / \Delta$ Exposed to Fluconazole.

Validation of Microarray Data by Real Time RT-PCR ............................................ 79

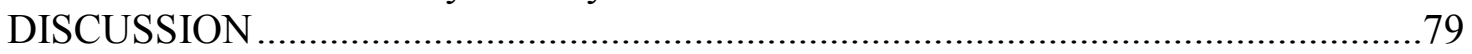

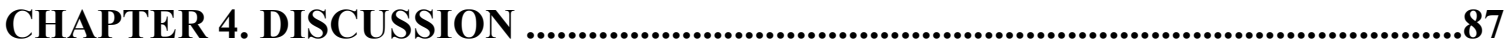

LIST OF REFERENCES..................................................................................................94

APPENDIX A. UPC2 SUPPLEMENTAL DATA..........................................................114

APPENDIX B. CAS5 SUPPLEMENTAL DATA ....................................................171

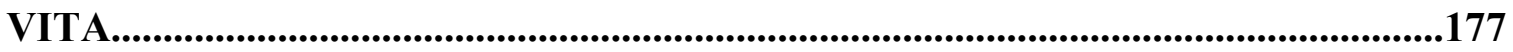




\section{LIST OF TABLES}

Table 2-1. C. albicans strains used in this study ...................................................18

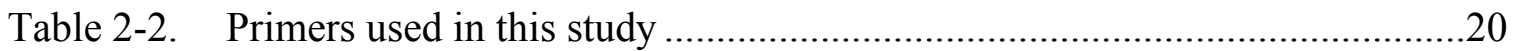

Table 2-3. MICs and MFCs in YPD in SC5314 background .....................................23

Table 2-4. MICs and MFCs in YPD in background of strains expressing resistance

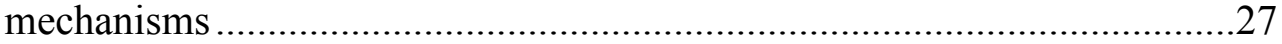

Table 2-5. MICs and MFCs in YPD of strains in 12-99 background .........................29

Table 2-6. Genes upregulated by at least 1.5-fold by fluconazole which are

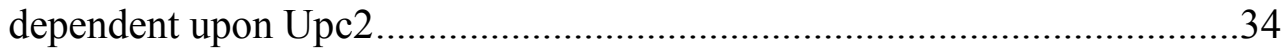

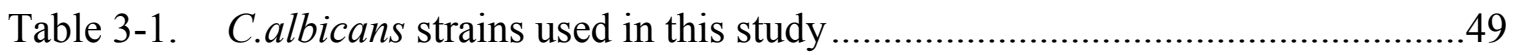

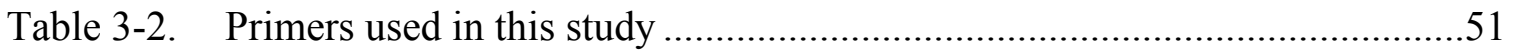

Table 3-3. Library hits with reduced MICs and MFCs in both RPMI and YPD ...........55

Table 3-4. MICs and MFCs in YPD in SC5314 background .....................................56

Table 3-5. MICs and MFCs in YPD in background of clinical resistant strains...........62

Table 3-6. MICs and MFCs in YPD in background of strains expressing resistance

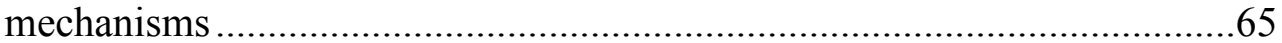

Table 3-7. Genes upregulated by at least 1.5-fold by fluconazole which are dependent upon Cas5

Table 3-8. Genes upregulated by at least 1.5-fold by fluconazole which are dependent upon Cas5 and Upc2.

Table A-1. Genes upregulated by at least 1.5 -fold by fluconazole in SC5314 ............114

Table A-2. Genes downregulated by at least 0.5 -fold by fluconazole in SC5314 .......156

Table B-1. TF disruptant clones displaying a reduction in FLC MIC in RPMI, rescreened in YPD.

Table B-2. Genes downregulated by at least 0.5 -fold by fluconazole which are dependent upon Cas5 and Upc2 


\section{LIST OF FIGURES}

Figure 2-1. UPC2 impacts fluconazole susceptibility in SC5314 background

Figure 2-2. UPC2 impacts fluconazole susceptibility in background of strains expressing resistance mechanisms .......................................................28

Figure 2-3. UPC2 impacts fluconazole susceptibility in 12-99 background ...................30

Figure 2-4. Expression of ERG11, CDR1, CDR2, and MDR1 as measured by qRTPCR

Figure 2-5. Validation of fluconazole-inducible and Upc2-dependent iron gene expression by qRT-PCR.

Figure 2-6. Sterol biosynthesis pathway in C. albicans

Figure 3-1. Effects of CAS5 and RPN4 on fluconazole susceptibility in SC5314 background.

Figure 3-2. $M K C 1$ effects fluconazole susceptibility in SC5314 background .60

Figure 3-3. Effect of fluconazole on CAS5 in 12-99 by time-kill assay .64

Figure 3-4. CAS5 slightly impacts fluconazole susceptibility in background of strains expressing resistance mechanisms

Figure 3-5. Expression of $C D R 1, C D R 2, E R G 11$, and $M D R 1$ by qRT-PCR .68

Figure 3-6. Validation of fluconazole-inducible and Cas5-dependent gene expression by qRT-PCR .80

Figure 4-1. An alternate sterol biosynthesis pathway in C. albicans .88

Figure 4-2. High-affinity ion uptake in yeast .90 


\section{LIST OF ABBREVIATIONS}

\begin{tabular}{|c|c|}
\hline $\mathrm{ABC}$ & ATP binding cassette \\
\hline AIDS & Acquired immune deficiency syndrome \\
\hline $\mathrm{AmB}$ & Amphotericin B \\
\hline AP-1 & Activator protein-1 \\
\hline APC & Antigen presenting cell \\
\hline ARE & AP-1 recognition element \\
\hline bHLH & Basic helix-loop-helix \\
\hline bZip & Basic leucine zipper \\
\hline c-CRD & carboxyl terminus cysteine rich domain \\
\hline cDNA & Complementary deoxyribonucleic acid \\
\hline CDR & Candida drug resistance \\
\hline $\mathrm{CFU}$ & Colony forming units \\
\hline CLSI & Clinical and Laboratory Standards Institute \\
\hline CRD & Cysteine rich domain \\
\hline cRNA & Complementary ribonucleic acid \\
\hline CWI & Cell wall integrity \\
\hline DBD & DNA binding domain \\
\hline DNA & Deoxyribonucleic acid \\
\hline DRE & Drug response element \\
\hline Etest & Epsilometer test \\
\hline FLC & Fluconazole \\
\hline FRT & FLP recognition target \\
\hline GEO & Gene Expression Omnibus \\
\hline GI & Gastrointestinal track \\
\hline GO & Gene Ontology \\
\hline GOF & Gain-of-function \\
\hline GOI & Gene of interest \\
\hline HAART & Highly active antiretroviral therapy \\
\hline HIV & Human immunodeficiency virus \\
\hline $\mathrm{HOG}$ & High osmolarity glycerol \\
\hline ICU & Intensive care unit \\
\hline IV & Intravenous \\
\hline MAPK & Mitogen activated protein kinase \\
\hline MDR & Multidrug resistance \\
\hline MFC & Minimum fungicidal concentration \\
\hline MFS & Major facilitator superfamily \\
\hline MIC & Minimum inhibitory concentration \\
\hline n-CRD & Amino terminus cysteine rich domain \\
\hline Nou & Nourseothricin \\
\hline $\mathrm{Nou}^{\mathrm{R}}$ & Nourseothricin-resistant \\
\hline $\mathrm{Nou}^{\mathrm{S}}$ & Nourseothricin-susceptible \\
\hline OD & Optical density \\
\hline OPC & Oropharyngeal candidiasis \\
\hline
\end{tabular}




$\begin{array}{ll}\text { ORF } & \text { Open reading frame } \\ \text { PAMP } & \text { Pathogen-associated molecular pattern } \\ \text { PCR } & \text { Polymerase chain reaction } \\ \text { PDA } & \text { Potato dextrose agar } \\ \text { PKA } & \text { Protein Kinase-A } \\ \text { PRR } & \text { Pattern recognition receptor } \\ \text { qRT-PCR } & \text { Quantitative real time-polymerase chain reaction } \\ \text { RNA } & \text { Ribonucleic acid } \\ \text { RPMI } & \text { Roswell Park Memorial Institute } \\ \text { SBI } & \text { Sterol biosynthesis inhibitors } \\ \text { SD } & \text { Sabouraud dextrose } \\ \text { SRE } & \text { Sterol response element } \\ \text { TF } & \text { Transcription factor } \\ \text { TLR } & \text { Toll-like receptor } \\ \text { UV } & \text { Ultra Violet } \\ \text { VVC } & \text { Vulvovangial candidiasis } \\ \text { YPD } & \text { Yeast peptone dextrose } \\ \text { YRE } & \text { yAP-1 response element } \\ \text { 5-FOA } & \text { 5-Fluoroorotic acid }\end{array}$




\title{
CHAPTER 1. INTRODUCTION
}

\author{
CANDIDA ALBICANS
}

\section{Physiology}

Candida albicans is an opportunistic fungal pathogen commonly found as a commensal organisms in the oropharyngeal cavity, gastrointestinal track, and vagina of up to $75 \%$ of humans $(1,2)$. It is a dimorphic fungus and exists as a blastospore (or yeast cell) at temperatures below $35^{\circ} \mathrm{C}$ with glucose as a carbon source and in a filamentous cell when above $35^{\circ} \mathrm{C}$ and in the presence of serum (3). Blastospores are spherical, single cells that undergo mitosis by budding. After the completion of mitosis, a septum forms between the two cells and separation results in two asymmetrical mother and daughter cells similar to Saccharomyces cerevisiae (4). Under filamentous conditions, $C$. albicans can form hyphae or pseudohyphae. The formation of hyphae occurs when a blastospore induces the elongation site and subsequently extends a germ tube and forms a septum without constrictions after mitosis, but the cells do not separate (5). Instead, the hypha continues to elongate. Pseudohyphae are formed when a blastospore buds but the cells remain attached to each other after the formation of the septum, and the daughter cell subsequently elongates into a shape resembling a hypha. As the cells undergo mitosis, this process repeats with each terminal cell resulting in a chain of elongated cells (4-6).

The ability of $C$. albicans to change its morphology between these forms is considered to be a virulence factor, promoting disseminated infection and forming biofilms, and is regulated by various environmental signals (7-10). Although it typically colonizes the mucocutaneous surfaces, the hyphal phase is able to invade into deeper tissues and cause damage, particularly when the host defenses are compromised (11). $C$. albicans switches back to yeast cells once in the bloodstream in order for dissemination (12). These morphologies also play a role in the formation of biofilms, which typically occur on the surfaces of indwelling devices such as catheters and dentures. Biofilms begin forming when blastospores adhere to these devices and then form a thick hyphal layer, which subsequently disperses more fungal cells (13). Biofilms have been shown to be resistance to antifungal drugs and is due to prevention of drug penetration by the extracellular matrix, drug efflux, and persister cells within the biofilms (14-18).

The yeast to hyphal transition, which occurs during fungal invasion, also significantly alters the composition of the cell wall. The outermost layer of the Candida cell wall is composed of mannans and mannoproteins, while the inner layer is composed of $\beta-1,3$-glucan and chitin. Although the outer layer of mannan masks underlying cell wall components such as $\beta-1,3$-glucan, they become exposed during hyphal transition at the bud scars and also by removal of this outer layer by heat inactivation, antifungal drugs, or mutations in the mannan layer (19-25). These cell wall components are the main source of pathogen-associated molecular patterns (PAMPs) recognized by the 
pattern recognition receptors (PRRs) of the cells of the immune system, and their interaction is critical for establishing an anti-Candida immune response (26).

\section{Genetics}

C. albicans is a diploid yeast with no haploid stage during its cell cycle. Sequencing of its genome shows that it possesses 6,354 putative genes contained in 8 chromosomes (27). It lacks a conventional sexual cycle and instead its reproduction is mostly clonal. There does exist a mating type locus on chromosome 5, but the occurrence of meiotic recombination is infrequent (28).

Due to its lack of a traditional sexual cycle or a haploid stage, the diploid state of C. albicans makes it difficult to isolate homozygous mutants carrying recessive mutations. In the past, mild mutagenesis only resulted in the generation of auxotrophs $(29,30)$. Combining mild mutagenesis with ultraviolet light increases the types of mutations, but also generates unwanted genomic alterations and therefore genetically undefined mutants. Reliable methods for gene disruption have been developed which utilize electroporation transformation by homologous recombination, which occurs with a plasmid-resident gene, and results in chromosomal integration and ensures stable inheritance.

Auxotrophic strains were developed for use in transformation experiments, and the most common marker used is $U R A 3$ because of the ease of selection for the correct transformants. The URA-blaster method of gene disruption uses this marker flanked by direct repeats of bacterial hisG sequences. The cassette is constructed to contain the 5' and 3 ' regions of the targeted gene on either side of the his $G$ sequences. Upon transformation into a $\mathrm{ura}^{-}$strain, one allele is replaced with the cassette by homologous recombination. Positive selection for the presence of the disruption cassette in the first allele occurs by growth on media lacking uridine. Counterselection then occurs by plating on media containing 5-fluorooronic acid (5-FOA), which only allows for the growth of $\mathrm{ura}^{-}$strains which arise by homologous recombination between the his $G$ sequences. The presence of the URA3 gene, encoding orotidine 5'-phosphate decarboxylase, results in the conversion of 5-FOA to 5-fluorouracil, which is toxic to the cell. The counterselected heterozygous cells then undergo a second round of transformation and selection for disruption of the second allele. However, auxotrophic strains exhibit decreased virulence and limit the background from which genes may be deleted. Additionally, repeated use of this method leaves behind his $G$ sequences, providing homology sites for unwanted integration events (31).

Transformation methods using a dominant resistance marker suitable for routine transformation of clinical isolates would be ideal. Two such methods have been developed independent of auxotrophy. These methods utilize dominant resistance markers conferring resistance to either mycophenolic acid or nourseothricin which are fused to a site-specific $F L P$ recombinase, modified from $S$. cerevisiae, controlled by a $S A P 2$ or MAL2 promoter respectively. The markers are flanked by direct repeats of the 
FLP recognition target (FRT) and cassettes are constructed to contain the 5' and 3' regions of the targeted gene on either side of the FRT sites. After integration into the first allele, the promoter is induced and the FLP recombinase removes the dominant marker and FLP sequences. A second round generates a mutant which differs from its background strain only by the lack of a gene and the presence of FRT sequences (32-34).

\section{Epidemiology of Infection}

In recent decades, fungi have emerged as a major cause of disease particularly for individuals who are immunocompromised or hospitalized with a serious underlying disease (35-39). This increased incidence of infections caused by fungi correlates to the growth of the immunocompromised and immunosuppressed patient population. Immune defects caused by hematopoietic stem cell transplantation, solid organ transplantation, major surgery, HIV/AIDS, neoplastic disease, immunosuppressive therapy, advanced age, or premature birth are all high risk factors for fungal infections $(37,38,40-44)$.

The most important opportunistic fungal pathogen is the Candida spp. and are the fourth leading cause of nosocomial blood stream infections in the United States (45). As stated previously, Candida spp. are common commensal organisms in the oropharyngeal cavity, gastrointestinal track, and vagina of humans. This organism is capable of opportunistic infection upon disruption of the normal flora by broad spectrum antimicrobial treatment, a breach in the mucocutaneous barrier due to surgery or the presence of catheters, or defects in the host immune system caused by neutropenia, HIV/AIDS, chemotherapy, and organ or bone marrow transplantation (2).

The most common fungal infections that occur are invasive and mucosal infections. Invasive candidiasis can occur in the bloodstream or in one or more organs and candidemia is the most common form of invasive candidiasis (45), typically occurring in hospitalized patients with serious underlying conditions. The incidence of nosocomial candidiasis has steadily increased over the past three decades along with the widespread use of central venous catheters, broad spectrum antimicrobial agents, corticosteroids and cytotoxic chemotherapeutic agents. Such immunosuppression impairs the ability to fight opportunistic infections. Patients with candidemia are twice as likely to die during hospitalization as compared to noncandidal blood stream infections (46), with $10-49 \%$ mortality $(47,48)$.

Mucosal candidiasis mainly presents as vulvovaginal candidiasis (VVC) or oropharyngeal candidiasis (OPC). VVC typically occurs in women of childbearing age, but also occurs due to antibiotic use, immunosuppressive therapy, hormone replacement therapy, and diabetes mellitus (49). OPC can occur in elderly patients with dentures, providing a surface on which Candida can adhere, which is resolved by removal of the dentures and proper dental hygiene $(50,51)$. In AIDS patients, OPC is most the commonly encountered opportunistic infection (52-54). Such an infection occurs on the soft and hard palates, pharynx, tongue, and buccal mucosa, and is typically the first 
clinical sign of an underlying HIV infection (55). The occurrence of OPC increases as $\mathrm{CD}^{+}$cells decrease below 200 cells $/ \mathrm{mm}^{3}$ (56).

The increasing susceptibility of HIV/AIDS patients to opportunistic fungal infections occurring alongside the loss in $\mathrm{CD}^{+}$cells implicates that the cytokines produced by these cells are important for immunity to Candida (57). Indeed, functional cell-mediated immune responses are important for the host's defense against Candida spp. This is made evident by the engagement of PRRs on antigen presenting cells (APCs) by Candida, resulting in the secretion of specific cytokines which in turn promote the differentiation of activated $\mathrm{CD}^{+}{ }^{+} \mathrm{T}$ cells into Th17 cells (58). These cells express the cytokine IL-17 and signaling through the IL-17 receptor (IL-17R) is crucial for an effective anti-Candida immune response (59).

Due to their enhanced susceptibility, up to $90 \%$ of all AIDS patients contract OPC and many die as a direct consequence (56). A significant decrease in HIV-related opportunistic infections, including OPC, occurred upon the advent of highly active antiretroviral therapy (HARRT). However, significant morbidity associated with Candida continues for the HIV/AIDS patient population due to high cost, insufficient monitoring, and poor patient compliance $(55,60)$.

\section{Treatment of Candida Infections}

Despite the wide spectrum of disease caused by $C$. albicans, there are few fundamentally different classes of clinical antifungals available for treatment due to limited unique and important fungal-specific targets which are not found in the host (61). Currently available antifungal drugs act primarily on the fungal cell wall and ergosterol found in the cell membrane. The fungal cell wall displays an electron-dense fibrillar outer layer composed of mannoproteins and an inner layer of glucans (such as $\beta-1,3-$ glucan) and chitin which confer elasticity and tensile strength to the cell wall $(62,63)$. Ergosterol is found in the fungal cell membrane and regulates membrane fluidity and asymmetry, and ultimately membrane integrity and transport across the membrane (64). Perturbation of either of these components impacts the fungal cell's survival. Both components are exploitable drug targets as the cell wall is essential for survival, and human cells lack both cell walls and ergosterol. The currently available antifungals which are primarily used for treatment include the polyenes, the azoles and the echinochandins (65).

Polyenes, such as amphotericin B (AmB), directly bind to membrane ergosterol and as a result cause impairment of yeast cell physiology (66-71). Channel formation and subsequent membrane permeabilization is a secondary mechanism that enhances the antifungals activity (72). It has a broad spectrum of action including most major fungal pathogens and was the sole drug available to control fungal infections for 30 years (73). For oral infection its use is usually limited to topical application as it is not readily absorbed from the gut (74). It is primarily given intravenously (IV) for systemic infections but has been associated with significant nephrotoxicity. The development of 
lipid formulations allowed for higher doses with less toxicity but they are more costly (75-77). Despite its fungicidal activity, cases of resistance have occasionally been reported (78).

The azoles represented a major advancement in ability to safely and effectively treat local and systemic fungal infections, and their high safety profile led to extensive use $(65,79)$. Fluconazole (FLC) inhibits ergosterol production essential in cell membrane formation (80) by targeting the product of ERG11, a critical cytochrome $\mathrm{P} 450$ enzyme of the ergosterol biosynthesis pathway known as lanosterol $14 \alpha$-demethylase which demethylates lanosterol (81). Inhibition of this enzyme results in depletion of ergosterol and accumulation of toxic sterol intermediates including $14 \alpha$-methylated sterols which alters membrane structure and function, and ultimately disrupts its integrity $(79,82)$. The azoles are available in both IV and oral formulations, and are currently the only oral option. Oral administration of fluconazole undergoes relatively little metabolism, is absorbed rapidly, is less toxic than AmB and is relatively inexpensive (77, $80,83,84)$. One drawback is its fungistatic activity instead of being fungicidal $(61,85)$ and as a result facilitates the development of resistance (86-88).

The echinochandins, such as caspofungin, are the most recent class of antifungal drugs. They act by inhibiting $(1,3)-\beta$-D-glucan synthase which is required for glucan synthesis, an important structure in the fungal cell wall (77). This class of antifungal represents significant advancement in antifungal therapy attributed to their fungicidal activity, proven clinical efficacy, and limited toxicity and drug-drug interactions. However, they are only available IV and are associated with moderate cost which somewhat limits their utility.

\section{FUNGISTATIC AZOLE ACTIVITY AND AZOLE RESISTANCE}

\section{Clinical Impact of Fungistatic Azole Activity}

As mentioned above, the fungistatic nature of azoles limits their efficacy $(61,85)$. The fungal cells are able to grow despite the inhibitory effects of the antifungal agent (61). Therefore, the inability to kill fungal cells may facilitate the development of resistance creating problems for treatment, especially for immunocompromised patients with OPC $(89,90)$. OPC is typically treated with low doses of fluconazole $(100 \mathrm{mg} /$ day $)$ over a long period of time. Upon the occurrence of a relapse, the dosage is increased (up to $800 \mathrm{mg} /$ day).

The incidence of OPC has decreased with the use of HARRT. However despite this advancement, resistance has been shown to still occur and many resistance mechanisms are maintained even after the antifungal agent is no longer present (61). This suggests that for the treatment of fungal infection it is important that the antifungal agent have enhanced abilities (91-93). As a result the development of novel co- 
therapeutic agents imparting enhanced activity upon the azoles would represent a favorable strategy by which to advance treatment (90, 94-96).

\section{Response Mechanisms That Allow Fungistatic Azole Activity}

Azole antifungal inhibition of ergosterol biosynthesis pathway is not lethal in $C$. albicans, suggesting that coping mechanisms exist such as specific stress responses which may circumvent azole activity and permit survival of the fungal cells in the presence of the azoles. Indeed, C. albicans activates specific pathways in response to external stresses. Response mechanisms implicated in the fungistatic effect of the azoles include: increases in drug efflux pump and ergosterol biosynthesis gene expression (97), the Ras-cAMP-PKA pathway (98), the calcineurin pathway (99-101), the Ssk1p/Chk1p pathway (102), and the cell wall integrity (CWI) pathway (103-106).

Upon ketoconazole exposure, expression of genes involved in drug efflux pump (CDR1, CDR2, MDR1) and ergosterol biosynthesis (NCP1, MCR1, CYB5, ERG2, ERG3, $P O T 14, E R G 25, E R G 251)$ were induced (97). These efflux pumps are typically expressed as low or undetectable levels and become upregulated in the presence of certain chemicals. Increased expression of these drug efflux pumps reduces the intracellular concentration of drug and therefore decreases its effect. Also, many of the ergosterol biosynthesis genes identified to be induced upon ketoconazole exposure exist downstream from Erg11p, the azole target, and respond to ergosterol depletion by facilitating the production of alternate sterols including lanosterol, eburicol, obtusifoliol, $14 \alpha$-methyl fecosterol, and 14 $\alpha$-methylergosta-8,24(28)-dien-3 $\beta, 6 \alpha$-diol to be inserted into the cell membrane (107). Integration of these sterols into the plasma membrane disrupts its integrity resulting in its altered structure and function $(79,82,108)$. Under these conditions, growth is inhibited but the organism remains viable (109).

Hsp90 is a molecular chaperone that stabilizes the regulators of many eukaryotic cellular signaling pathways, and its inhibition disrupts many cellular processes (110, 111). Interestingly, compromise of its function blocks the development of azole resistance and renders drug-resistant isolates susceptible (112), such as a resistant $\operatorname{erg} 3 \Delta / \Delta$ mutant (113). It has also been shown to improve the efficacy of treatment in a murine model of disseminated infection (114). Of the many pathways in which Hsp90 interacts, it has been shown to play roles in some of the pathways implicated in the response to azoles.

Ras1 is a small GTPase that regulates the cAMP-protein kinase A (PKA) pathway controlling morphogenesis in response to nutrient limitation, $\mathrm{pH}, \mathrm{CO}_{2}$, and serum (12). In response to these stimuli, the guanine exchange factor Cdc 25 activates Ras 1 to its GTP-bound state which continues on to stimulate Cdc35 and cAMP-associated protein (Cap1/Srv2) for the production of cAMP $(115,116)$. This increase in intracellular cAMP activates the catalytic subunits of PKA which activates the transcriptional regulator of morphogenesis, Efg1 $(117,118)$. Also playing a role in the transition from yeast to hyphal growth is the Hsp90 molecular chaperone. Pharmacological disruption of Hsp90 
function induces hyphal growth even in the absence of external cues, and therefore is a negative regulator of the Ras-cAMP-PKA pathway (9). Disruption of the genes encoding adenyl cyclase (Cdc35) or Cap1/Srv2 renders the cells hypersusceptible to the azoles, with a fungicidal effect. This has been correlated to the fungal cell's inability to upregulate a multidrug transporter gene $C D R 1$ (98), known to pump fluconazole out of the cell.

Calcineurin is a protein phosphatase consisting of a catalytic A (Cna1) and a regulatory $\mathrm{B}(\mathrm{Cnb} 1)$ subunit which are highly conserved between yeasts and mammals (119). The molecular chaperone Hsp90 has been shown to interact with the Cna1 subunit, stabilizing the protein for activation in response to stress $(99,120,121)$. In response to stress, calcineurin dephosphorylates downstream transcriptional regulators, including the transcription factor Crzl which regulates genes involved in cell wall biosynthesis and ion homeostasis (122-125). Calcineurin is implicated as being required for the fungistatic activity of the azoles $(100,126)$. Hsp90 or calcineurin compromise imparts fungicidal activity upon the azole antifungals $(114,122,124)$. Calcineurin pathway mutants disrupted for the calcineurin subunit genes $C N A 1$ and $C N B 1$ treated in combination with an azole and cyclosporine A (which inhibits calcineurin) also render the azoles fungicidal. This effect does not involve changes in the expression of the transporter genes (99-101).

The high osmolarity glycerol (HOG) pathway is mediated by the Hog1 mitogen activated protein kinase (MAPK) in response to osmotic and oxidative stress (127-129). It has also been implicated in morphogenesis and cell wall formation. Hog1 is phosphorylated by the Pbs2 MAP kinase downstream from the Ssk1p/Chk1p pathway (102). Environmental signals cause the Chk1 histidine kinase to autophosphorylate which then dephosphorylates Ssk1 and stimulates the Pbs2-Hog1 MAPK cascade (130). Disruption of either genes encoding the response regulator (Ssk1p) or histidine kinase (Chk1p) caused the fungal cells to become hypersusceptible to the azoles due to the inability to pump out the drug resulting in fluconazole accumulation within the cells (102). Additionally, when CHK1 is disrupted, the properties of the cell surface are altered along with lowered glucan content (131-133). On the other hand, disruption of HOG1 did not result in altered susceptibility to fluconazole, but did result in resistance nikkomycin $\mathrm{Z}$ and Congo red (127).

In S. cerevisiae, the cell wall integrity MAP kinase (CWI MAPK) pathway is the major contributor to cell wall assembly and maintenance (85). Upon sensing stress at the surface of the fungal plasma membrane, the linear MAPK cascade amplifies the initial signal for transmission to downstream transcriptional regulators such as Rlm1p. This regulator is responsible for the majority of the transcriptional output produced by this pathway $(63,103)$. This pathway in conserved in C. albicans and has been previously suggested to be critical for the fungistatic activity of sterol biosynthesis inhibitors (104). $\mathrm{CaCAS5}$ encodes a transcriptional regulator of the cell wall damage response and is postulated to be the functional equivalent of ScRlmlp (134). When the cell wall integrity pathway is disrupted, specifically by disrupting the kinase genes $P K C 1$ and $M K C l$, the cells exhibit increased fluconazole susceptibility $(103,106)$. The upstream protein kinase 
$\mathrm{C}, \mathrm{Pkc1p}$, phosphorylates the MAPK Mkc1p in response to fluconazole, but is interrupted by its absence. Hsp90 depletion was also shown to destabilize Mkclp in the same study, therefore blocking $P K C 1$-signaling. Additionally, deletion of $P K C 1$ in a resistant $\operatorname{erg} 3 \Delta / \Delta$ mutant resulted in enhanced susceptibility (106).

The transcription factors and their target genes have yet to be identified for many of the pathways involved in the response to azole activity and there may be other yet to be identified pathways involved. Target genes of these pathways would be useful as cotherapeutic drug targets to improve the activity of azole against $C$. albicans.

\section{Emergence of Clinical Azole Resistance}

When an infection progresses or persists despite treatment with antifungals, this is known as clinical resistance. There are two forms of resistance. Organisms that are resistant prior to exposure to an antifungal agent have primary or intrinsic resistance while secondary resistance arises after initiation of antifungal treatment (77). Due to their fungistatic activity and widespread use in the treatment of fungal infections, antifungal drug resistance most commonly occurs for the azoles. Their fungistatic nature makes it difficult to completely clear all of the fungal cells, especially in an immunocompromised individual, resulting in reoccurring infection.

The emergence of fluconazole resistance is attributed to many factors. First, Candida infections are not usually due to newly acquired resistant strains, but rather commensal strains that either become more virulent or that no longer have to compete with other normal flora (135). The use of low doses of fluconazole over extended periods of time also creates an ideal environment for the selection of resistant strains. Transmission between individuals also can occur, although typically among immunocompromised individuals $(136,137)$.

In order to characterize fluconazole susceptibility in the clinical setting, the Clinical and Laboratory Standards Institute (CLSI) Subcommittee for Antifungal Susceptibility Testing established standard breakpoints: sensitive $\leq 2 \mu \mathrm{g} / \mathrm{ml}$, susceptibledose dependent (S-DD) $=4 \mu \mathrm{g} / \mathrm{ml}$, resistant $\geq 8 \mu \mathrm{g} / \mathrm{ml}$ (138). Appropriate measurement of resistance in vitro is often correlated with treatment failure (139). However, there are many others factors that can impact the likelihood of therapeutic success such as clinical status of the patient, presence of foreign material, and location of infection $(79,140)$.

Due to the rise in the immunosuppressed patient population the usage of antifungal agents has also increased, coincidently along with a rise in resistance. The azoles are the most important antifungal agent because they are relatively safe, can be orally delivered, and are relatively inexpensive. Fluconazole is the most widely used azole for treating OPC, which is the most common AIDS-associated opportunistic infection $(141,142)$. Repetition and lengthy duration of therapy for OPC in AIDS patients has led to an increased incidence of treatment failures secondary to the emergence of azole resistance $(77,89,143,144)$. Recurrent OPC most often occurs 
when $\mathrm{CD}^{+} \mathrm{T}$ cells drop below $50 \mathrm{cells} / \mathrm{mm}^{3}$. In fact, one study identified $33 \%$ of latestage AIDS patients with drug-resistant Candida present in their oral cavities (145).

Other patient populations are increasingly exposed to azoles for treatment and prophylaxis including those undergoing aggressive cancer therapy, surgical intensive care unit (ICU) patients, nonneutropenic patients, and women with VVC (146-150).

However, the increased use of the azole antifungals in the immunocompromised patient population has the largest effect on the frequency, morbidity, and overall response to therapy of fungal infections.

It is logical for resistance to antifungal agents to develop as a result of their use

for treatment. An additional complication of fluconazole resistance is the potential to develop cross-resistance to other azoles and the polyenes, as the activities of both antifungals are associated with ergosterol. As a result, the ability to limit the emergence of resistance to these agents and the development of more potent drugs is clinically important. Strategies to avoid and suppress resistance have yet to be defined, but one suggested method is to utilize combination therapy in order to broaden efficacy and preserve the utility of the azole class of antifunagls (151).

\section{Molecular Mechanisms of Resistance to Azoles}

Several mechanisms of azole resistance have been documented in C. albicans. One method involves the export of the azole antifungal out of the cell by constitutive overexpression of efflux pumps, preventing the intracellular accumulation of effective concentrations. At least two efflux systems exist in C. albicans and have been found to be overexpressed in resistant isolates: the ATP-binding cassette superfamily (ABC) and the major facilitator superfamily (MFS) (152-156).

$\mathrm{ABC}$ transporters rely on the binding and hydrolysis of ATP for energy and have a broad specificity including many of the azoles (157). Two ABC transporters, encoded by $C D R 1$ and $C D R 2$, have been implicated in azole resistance. In clinical matched isolates, increased expression of $C D R 1$ and $C D R 2$ is correlated with decreased azole susceptibility $(155,156,158)$. This was further demonstrated by experimentally controlling overexpression of $C D R 1$ which conferred resistance to fluconazole (159). Deletion of $C D R 1$ from a clinical isolate with resistance to several azoles resulted in a large reduction in resistance, while deletion of $C D R 2$ had a lesser effect (160).

Unlike ABC transporters, MFS transporters rely on the energy of the proton motive force generated by the proton gradient across the cell membrane and are relatively specific for fluconazole and voriconazole (161). The main MFS transporter identified to contribute to fluconazole resistance is Mdr1. Increased expression of $M D R 1$ has been detected in resistant isolates when compared to their susceptible matched pairs (152, 158). Experimental overexpression of $M D R I$ was unable to confer fluconazole resistance, because higher levels of $M D R 1$ expression than what were achieved are necessary to confer resistance to fluconazole (162). The role of $M D R 1$ in fluconazole 
resistance was confirmed when deletion from an $M D R 1$-overexpressing resistant isolate resulted in decreased fluconazole resistance (33).

Other mechanisms by which azole resistance develops involve changes in lanosterol demethylase or other components of the ergosterol biosynthesis pathway. Inhibition of lanosterol demethylase by the azoles results in accumulation of the methylated toxic sterol 14 $\alpha$-methylergosta-8,24(28)-dien-3 $\beta, 6 \alpha$-diol which inhibits fungal cell growth (108). In order to compensate for this loss-of-function inhibition due to azole activity, there exists a bypass pathway through the inactivation of $E R G 3$ product, $\Delta^{5,6}$ desaturase. This results in the accumulation of alternate, nontoxic sterols (such as fecosterol) in place of the toxic sterols, allowing the organism to circumvent inhibited cell growth, and results in azole resistance $(108,163)$.

Drug target alterations also lead to resistance by decreased drug affinity, while still maintaining an active target. White et al. analyzed the ERG11 sequences of 17 sequential isolates which develop resistance and identified a point mutation resulting in a substitution between two residues known to be involved in interacting with the heme in the active site $(156,164,165)$. Such point mutations in ERG11 may result in conformational changes that prevent effective binding between azoles and their target without affecting function of the enzyme in ergosterol biosynthesis $(165,166)$. Several point mutations have been identified in resistant isolates and lead to decreased azole susceptibilities (165-167).

Overexpression of ERG11 is another mechanism of resistance and results in overproduction of the target enzyme, lanosterol demethylase. This increased production overwhelms the intracellular azole concentration such that the drug does not inhibit the reaction completely $(152,156,167)$. ERG11 upregulation is found to be present along with one of the efflux pump resistance mechanisms in at least $25 \%$ of clinical resistant isolates (167).

All of these mechanisms impact reduced azole susceptibility, but high-level azole resistance appears to accumulate gradually over time with the acquisition of multiple mechanisms $(167,168)$. Sanglard, et al. (169) studied set of 16 sequential C. albicans isolated from five AIDS patients, selected based on their increasing fluconazole resistance following prolonged treatment. The observed decreased in fluconazole accumulation was shown to be associated with a 10-fold increase in mRNA levels of $M D R 1$ (normal levels for $C D R 1$ ). Additionally, White, et al. examined 17 sequential isolates from an HIV patient and found increases in ERG11, MDRI and CDR1, further suggesting that high-level azole resistance results from contributions of several mechanisms (156). 


\section{TRANSCRIPTIONAL REGULATORS}

\section{Transcription Factor Classes}

Two major classes of transcription factors are involved in drug resistance, the first being the basic leucine zipper (bZip) proteins. The bZip class is found only in eukaryotic cells and is characterized by a highly conserved 60-80 amino acid long basic leucine zipper motif shaped in a bipartite $\alpha$-helix which binds the DNA major groove (170). The DNA binding domain (DBD) is located in the N-terminal portion of the bZip motif and consists of two basic amino acid clusters which are responsible for binding to DNA. The leucine zipper is located in the C-terminal portion and has leucine residues every 7 amino acids (170). It is through the leucine zipper that the bZip class proteins dimerize and bind DNA as either homodimers or heterodimers resulting in gene transcription activation or repression $(170,171)$.

Within the bZip class, there exist 13 different families of proteins. Two families, the Jun and the Fos, dimerize and form the activator protein-1 (AP-1) transcription factor family implicated in drug resistance (171). These proteins bind DNA as heterodimers with one Jun protein and one Fos protein or homodimers with two Jun proteins. The dimmers bind DNA at a specific AP-1 recognition element (ARE) motif which is found in the promoters and enhancers of genes involved in several cellular processes.

Fungal AP-1 transcription factors are referred to as yeast AP-1 (yAP-1) and bind to the yAP-1 response element (YRE) $(172,173)$. Yeast bZip transcription factors differ by being the only bZips to contain two cysteine rich domains (CRD) located in the amino terminus (n-CRD) and carboxy terminus (c-CRD) of the 3' bZip domain (174). These CRDs act in the localization and activation of yAP-1 transcription factors.

The largest class of eukaryotic transcriptional regulators is the zinc finger proteins (175), which are defined by a zinc finger motif, structured as one $\alpha$-helix and a pair of antiparallel $\beta$ strands, containing cysteines and histidines which bind zinc atoms for structure, function and stabilization. Within this class exist three families of zinc finger proteins: classical zinc fingers $\left(\mathrm{Cys}_{2} \mathrm{His}_{2}\right)$ bind DNA as monomers, $\mathrm{Cys}_{4}$ zinc fingers bind as either homodimers or heterodimers, and zinc cluster proteins $\left(\mathrm{Cys}_{6}\right)$ bind as monomers, homodimers and heterodimers. The zinc cluster transcription factors have six cysteines bound by two zinc atoms and are exclusive to fungi (175-177).

The zinc cluster transcription factors are typically found in the nucleus and many are constitutively bound to their promoters (175). These transcription factors are comprised of a DBD, regulatory domain, and acidic domain. Their DBD contains zinc finger motif, linker, and dimerization domains. The zinc finger motif binds as dimers in the DNA major groove, the linker designates DNA binding specificity, and the dimerization domain resembles the leucine zipper in bZip proteins $(175,178)$. Their regulatory domain is specific to zinc cluster transcription factors and upon its deletion, 
renders them constitutively active $(179,180)$. Phosphorylation or dephosphorylation of the acidic domain acts as the activation domain (175).

\section{Transcription Factors and Fungistatic Azole Activity}

Some of the transcriptional regulators of the stress responses which circumvent azole activity and permit survival in the presence of the azoles have been identified, but the direct downstream targets of many remain unclear.

EFG1 encodes a transcription factor of the basic helix-loop-helix (bHLH) class and is a member of the APSES family fungal-specific regulators $(181,182)$. The bHLH domain promotes dimerization and DNA binding to the Efg1 recognition sequence (EGR or E-box) box, which is found in many genes involved in morphogenesis. Indeed, EFG1 plays a role in the induction and repression of cell wall genes in yeast and hyphal forms (183). This agrees with its function downstream of the Ras-cAMP-PKA pathway. Upon disruption of $E F G 1$, an increase in membrane fluidity was observed concurrent with a decrease in ERG11 expression, rendering the cell hypersusceptible to fluconazole (184).

One of the downstream regulators of the calcineurin pathway is Crz1 (100). It is a classical $\mathrm{Cys}_{2} \mathrm{His}_{2}$ zinc finger and has been found to control the expression of several $\mathrm{Ca}^{2+} /$ calcineurin-responsive genes through CDRE sequences in their promoters (122, 185). It has also been documented that disruption of $C R Z 1$ results in hypersusceptibility to fluconazole $(100,122)$. Interestingly, disruption of the catalytic $\alpha$-subunit of protein kinase $\mathrm{CK} 2, C K A 2$, results in fluconazole resistance through overexpression of $C D R 1$ and $C D R 2$, but subsequent $C R Z 1$ disruption suppresses this phenotype (186).

In the $S$. cerevisiae CWI pathway, the transcription factor ScR $1 m 1$ plays a major role. This pathway is conserved in C. albicans and the functional homolog to ScRlm1 has been identified as Cas5, a classical $\mathrm{Cys}_{2} \mathrm{His}_{2}$ zinc finger protein $(134,187)$.

Microarray analysis in the presence of caspofungin identified the requirement of Cas 5 for the expression of many caspofungin-responsive genes, including those cell wall protein and cell wall maintenance genes. Motifs were also identified that may represent the Cas5-binding sites in the promoter regions of 10 out of 13 Cas5-dependent genes examined. Additionally, disruption of $C A S 5$ resulted in hypersusceptibility to caspofungin, Congo red, and sodium dodecyl sulfate (134). Due to its role in the CWI pathway, it is possible that it is also hypersusceptible to the azoles, similarly seen for other components of this pathway.

\section{Transcription Factors and Azole Resistance}

Constitutive overexpression of the efflux pumps involved in azole-resistance is due to constitutively active transcription factors, as a result of gain-of-function (GOF) mutations. A few of these transcription factors have been identified. 
The C. albicans bZip yAP-1 transcription factor, Cap1, is closely related to the $S$. cerevisiae Yap1 and has been identified to be involved in multidrug resistance (188). Caplp is primarily involved in protecting the cell against oxidative stress (189-191). Genome-wide location and expression analysis was undertaken in order to determine which genes Cap1 directly binds and regulates. Targeted groups included genes involved in osmotic stress response, response to drugs (including $M D R 1$ ), phospholipid transport, and nitrogen utilization (192). Cells disrupted for $C A P 1$ were unable to upregulate $M D R 1$ in response to fluconazole and the formation of a hyperactive Cap1, by disruption of the c-CRD, resulted in fluconazole resistance and increased MDR1 expression (189). Cap1 binds to the Yap1 response element (YRE) motif located in the MDR1 promoter, but the absence of this motif in a fluconazole-resistant clinical isolate did not alter MDR1 expression, suggesting there is another regulator controlling its expression during resistance $(193,194)$.

Constitutive $M D R 1$ overexpression resulting in multidrug resistance is due to GOF mutations in the zinc cluster transcription factor Mrrl. MRRl was identified as one of the genes upregulated in three $M D R 1$-overexpressing fluconazole-resistant isolates when compared to their fluconazole-susceptible matched isolates. These isolates were identified as being homozygous for mutated MRRl GOF alleles (195). Inactivation of this $M R R 1$ allele resulted in the loss of $M D R 1$ overexpression and increased fluconazole susceptibility. Deletion of MRR1 reduced fluconazole resistance more than MDR1, suggesting that additional Mrrlp-controlled mechanisms are involved. Meanwhile, introduction of the GOF allele into a fluconazole-susceptible background resulted in constitutive overexpression of $M D R l$ and multidrug resistance. Other transcription factors have been implicated in the upregulation of $M D R 1$, but Mrrl is the central regulator of $M D R 1$ expression in fluconazole resistance.

Taclp is a zinc cluster transcription factor is another transcription factor involved in regulating clinical azole-resistance (196). It binds the drug response element (DRE) consensus sequence located in the promoters of $C D R 1$ and $C D R 2(196,197)$. Disruption of $T A C 1$ abolishes $C D R 1$ and $C D R 2$ expression and is hypersusceptible to fluconazole. Azole resistance occurs when the isolates have become homozygous for a GOF TAC1 allele, likely because zinc cluster transcription factors act as dimers. Such a TAC1 allele was isolated from an azole-resistant strain which overexpressed $C D R 1$ and $C D R 2$, and when place in the background of a susceptible strain was still able to confer resistance and $C D R 1$ and $C D R 2$ overexpression. However, this resistance depends on the presence of $C D R 1$ and $C D R 2$ (198).

Upc2p is a zinc cluster transcription that controls inducible ergosterol gene expression in the presence of the azoles. When UPC2 is disrupted in a susceptible background, it becomes hypersusceptible to sterol biosynthesis inhibitors (SBIs), including fluconazole in addition to abolished $E R G$ gene transcription and reduced ergosterol levels $(199,200)$. It binds to a sterol response element (SRE) motif found in the promoter region of $E R G$ genes and also $U P C 2$ itself. Genome-wide location profiling identified the Upc2p gene groups targets involved in ergosterol biosynthesis (including $E R G 11)$, drug transport (MDR1), and transcription factors (UPC2) (201). The first GOF 
mutation in Upc2 was identified in matched isolates with no CDR1, CDR1, or MDR1 overexpression by Northern blot (152). Microarray analysis revealed that UPC2, ERG11 and MDR1 were overexpressed in the resistant isolate, containing a G648D GOF mutation (202). When the mutated GOF allele was introduced into a fluconazolesusceptible background, both ERG11 and MDRI were upregulated. Other UPC2 GOF mutations have also been identified to confer fluconazole-resistance and increased ERG11 expression (203-205).

\section{CENTRAL HYPOTHESIS AND RESEARCH OBJECTIVES}

C. albicans responds to antifungal stress through specific signal transduction and transcriptional activation pathways, which circumvent azole activity and permit survival of the fungal cells in the presence of the azoles. Several response mechanisms have been identified to mediate fluconazole susceptibility. Increased drug efflux pump expression results in decreased intracellular concentrations of the azoles. Increased ERG11 expression results in increased intracellular concentrations of lanosterol demethylase and therefore the amount of enzyme increases that is not bound by the azoles. Additionally, increased expression of other genes involved in the ergosterol biosynthesis pathway in response to ergosterol depletion leads to accumulation of alternate sterols which results in inhibited growth but the cell remains viable. Disruption of components of the RascAMP-PKA pathway results in decreased inducible $C D R 1$ gene expression resulting in fungicidal azole activity, while disruption of the transcriptional regulator, Efg1, results in decreased ERG11 expression and hypersusceptibility to fluconazole. Compromise of the calcineurin pathway renders the azole fungicidal and disruption of one possible downstream regulator, Crzl, results in fluconazole hypersusceptibility. Disruption of either Ssk1 or Chk1 upstream of the HOG pathway resulted in intracellular fluconazole accumulation and hypersusceptibility. Disruption of Mkc1 and Pkc1 of the CWI pathway results in increased susceptibility to the azoles. The downstream transcriptional regulators and their target genes have yet to be identified for many of these pathways and there may also be novel pathways which have yet to be identified.

I hypothesized that pathways centrally involved in the response mechanisms to azole activity, permitting survival in their presence, require key transcriptional regulators. With the completion of the $C$. albicans genome sequencing project many genes were identified to code for transcription factors, and microarray technology can be used to identify their downstream targets. By studying mutants deleted for specific transcription factors, their impact on fluconazole susceptibility can be elucidated and their fluconazoleinducible target genes can be identified. Among the transcription factors identified, we

found both previously described as well as novel roles for these transcriptional regulators in fluconazole susceptibility.

Upc2, a zinc cluster transcription factor, has been previously identified as the transcriptional regulator of the ergosterol biosynthesis pathway. The disruption of this transcription factor has also been shown to enhance susceptibility to fluconazole, while GOF mutations result in azole resistance. My first research objective (Chapter 2) was to 
examine susceptible and resistant strains disrupted for UPC2 by various susceptibility tests and gene expression analysis in an effort to elucidate the extent of its involvement in fluconazole susceptibility. I hypothesized that Upc2 is essential for fluconazole susceptibility in both susceptible and resistant backgrounds. Our studies showed that disruption of UPC2 enhanced susceptibility and overcame resistance by downregulation of $E R G$ genes, specifically $E R G 11$. Additionally, we identified a novel role for Upc 2 in regulating fluconazole-inducible iron homeostasis.

In research objective 2 (Chapter 3), we examined a library of mutants disrupted for genes encoding specific transcription factors in an effort to identify those which had enhanced susceptibility to fluconazole. I hypothesized that transcriptional regulators of known and possibly novel pathways would be essential for responding to azole activity. Our studies characterized the zinc finger transcription factor Cas 5 in regulating fluconazole susceptibility, through its role as the transcriptional regulator of the CWI pathway. Gene expression profiling identified genes involved in cell wall maintenance whose fluconazole-induced expression was dependent on CAS5. Additionally, we identified a novel role for Cas5 in iron homeostasis, similar to Upc2. 


\section{CHAPTER 2. UPC2 IS UNIVERSALLY ESSENTIAL FOR AZOLE RESISTANCE IN CANDIDA ALBICANS}

\section{INTRODUCTION}

The increased frequency of invasive fungal infections in recent decades is directly related to an expansion in the immunocompromised patient population including those with HIV/AIDS, cancer chemotherapy patients, neutropenic patients, recipients of transplants with indwelling catheters, and patients receiving antibiotics $(65,206,207)$. Candida species collectively are the fourth-leading cause of nosocomial infections in the United States and such infections are associated with unacceptably high rates of mortality $(65,208,209)$. Candida albicans is the most prevalent opportunistic human fungal pathogen and causes a wide variety of infections spanning from superficial, mucosal infections to invasive disseminated disease despite the availability of effective antifungal treatment. Moreover, the most common opportunistic infection among AIDS patients is oropharyngeal candidiasis (OPC), and chronic cases continue despite the availability of highly active anti-retroviral therapy (HAART) due to poor compliance, lack of access, and treatment failure of HAART $(52,53,210-212)$.

The azoles, particularly fluconazole, are the most widely used class of antifungals for the treatment of Candida infections $(65,79)$. Fluconazole acts by inhibiting the protein product of ERG11, lanosterol 14 $\alpha$-demethylase, which leads to ergosterol depletion and accumulation of toxic methylated sterol precursors resulting in inhibition of growth (213). In an immunocompromised host, the fungistatic nature of fluconazole limits its efficacy against this organism $(61,85)$. C. albicans exhibits inhibited growth in the presence of this fungistatic drug and it is generally believed that such fungistatic activity facilitates the development of resistance creating problems for treatment, especially in immunocompromised patients with OPC $(89,90)$. Currently known mechanisms of resistance to the azoles include alterations in the expression of drug efflux pumps (CDR1, CDR2 and MDRl) and ergosterol biosynthesis genes $E R G$, and mutations in $E R G 11$ (77). These mechanisms are frequently combined resulting in a stepwise development of resistance over time.

In C. albicans, UPC2 is a zinc-cluster transcription factor that regulates the expression of genes involved in ergosterol biosynthesis, including ERG11 (199, 214). Disruption of ERG11 or pharmacologic inhibition of the enzyme it encodes leads to reduced ergosterol production and the accumulation of alternate sterols including lanosterol, eburicol, obtusifoliol, $14 \alpha$-methyl fecosterol, and $14 \alpha$-methylergosta8,24(28)-dien-3 $\beta, 6 \alpha$-diol (107). Biosynthesis of these alternate sterols does not require $E R G 11$, but does require other genes in the ergosterol biosynthesis pathway, some of which appear to be regulated by Upc2. We reasoned that since Upc2 is required for the transcriptional activation of other genes of the sterol biosynthesis pathway, the disruption of UPC2 might result in enhanced activity of the azole antifungals in azole-resistant as well as -susceptible isolates. In the present study, we further examined the role of UPC2 in azole antifungal activity against both azole-susceptible and azole -resistant strains of 
C. albicans, in particular an azole-resistant clinical isolate and mutant strains that represent four of the major azole resistance mechanisms. Taken together, our results indicate that the $U P C 2$ transcriptional network is universally essential for fluconazole resistance in C. albicans.

\section{MATERIALS AND METHODS}

\section{Strains and Growth Conditions}

All C. albicans strains (Table 2-1) were stored as frozen stock in $40 \%$ glycerol at $-80^{\circ} \mathrm{C}$. YPD $(1 \%$ yeast extract, $2 \%$ peptone, and $1 \%$ dextrose $)$ agar plates and YPD liquid medium were used for routine growth of strains at $30^{\circ} \mathrm{C}$. For CFU counts during time-kill analysis, $\mathrm{PDA}(0.4 \%$ potato starch, $2 \%$ dextrose, and $1.5 \%$ agar) plates were used, and cultures grown on PDA were incubated at $35^{\circ} \mathrm{C}$.

\section{Drug Susceptibility Testing}

The minimum inhibitory concentrations (MICs) of fluconazole (FLC) were determined by using broth microdilution as described by the Clinical and Laboratory Standards Institute M27-A2, modified by using YPD media, and were read both visually and spectrophotometrically at 24,48 , and 72 hours. Minimum fungicidal concentrations (MFCs) were measured by removing $2 \mu \mathrm{l}$ from each well of the MIC plate and plating onto YPD agar. Also, serial dilutions from a $0.1 \mathrm{OD}_{600}$ suspension were diluted 4-fold and $2 \mu \mathrm{l}$ of each dilution plated onto YPD agar plates with and without $10 \mu \mathrm{g} / \mathrm{ml}$ fluconazole and then incubated at $30^{\circ} \mathrm{C}$ for 24 and 48 hours. Fluconazole activity was also assessed by Epsilometer test strips (Etest strips) (Biomerieux) according to manufacturer's instructions with the following modifications. A standardized cell suspension ( $0.5 \mathrm{McF}$ arland) was used to create a confluent lawn across YPD agar plates prior to Etest strip placement and then incubated at $30^{\circ} \mathrm{C}$ for 24 and 48 hours. Time-kill analyses were performed with a $0.5 \mathrm{McF}$ arland cell suspension, which was 10 -fold diluted into YPD media with and without $10 \mu \mathrm{g} / \mathrm{ml}$ fluconazole and incubated at $35^{\circ} \mathrm{C}$. Aliquots were removed at $0,6,12$, and 24 hours, 10 -fold serial diluted and plated onto PDA. CFUs were counted in duplicate after 48 hours at $35^{\circ} \mathrm{C}$ and plotted on a log-scale curve versus time (215).

\section{Construction of ERG11 Strains with Mutant Alleles}

CaERG11 coding sequences were amplified by PCR ( $P f u$ DNA polymerase; Stratagene) from C. albicans genomic DNA using the primers ERG11-A and ERG11-E. Products were cloned into pCR-BLUNTII-TOPO using a Zero Blunt TOPO PCR Cloning Kit (Invitrogen) and transferred into Escherichia coli TOP10 cells with selection on LB agar plates containing $50 \mu \mathrm{g} / \mathrm{ml}$ kanamycin. Plasmid DNA was purified (QIAprep; 
Table 2-1. C. albicans strains used in this study

\begin{tabular}{|c|c|c|c|c|}
\hline Strain & Designation & $\begin{array}{c}\text { Strain } \\
\text { Background }\end{array}$ & $\begin{array}{c}\text { Relevant Characteristics or } \\
\text { Genotype }\end{array}$ & Source \\
\hline SC5314 & SC5314 & $\mathrm{N} / \mathrm{A}$ & $U P C 2-1 / U P C 2-2$ & ATCC \\
\hline Clinical isolate 2-79 & $2-79$ & N/A & Susceptible isolate & Redding, 1994 \\
\hline Clinical isolate 12-99 & $12-99$ & N/A & Resistant isolate & Redding, 1994 \\
\hline UPC2M4A & upc $2 \Delta / \Delta$ & SC5314 & upc2-1 $1 \Delta:: \mathrm{FRT} / u p c 2-2 \Delta:: \mathrm{FRT}$ & Dunkel, 2008 \\
\hline UPC2M2K21A & $u p c 2 \Delta / \Delta+U P C 2$ & $\mathrm{UPC} 2 \mathrm{M} 2 \mathrm{~A}$ & upc2-1 $1 \Delta:: \mathrm{FRT} / U P C 2^{\mathrm{S} 1-1}-\mathrm{caSAT1}$ & Dunkel, 2008 \\
\hline 12-99UPC2A5C1A & $12-99 u p c 2 \Delta / \Delta$ & $12-99$ & upc $2 \Delta:: \mathrm{FRT} / u p c 2 \Delta:: \mathrm{FRT}$ & This study \\
\hline 10C1B1M1 & $E R G 11^{\mathrm{K} 143 \mathrm{R}}$ & SC5314 & $\begin{array}{c}E R G 11^{K 143 R}:: \mathrm{FRT} / E R G 11^{K 143 R}:: \mathrm{FRT} \\
U P C 2-1 / U P C 2-2\end{array}$ & This study \\
\hline 10C1B1M1UPC2C9H & $E R G 11$ upc $2 \Delta / \Delta$ & 10C1B1M1 & 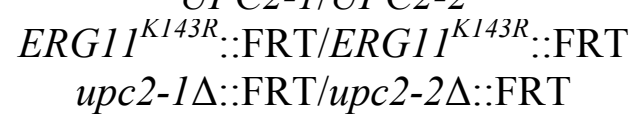 & This study \\
\hline SCMRR1R34A & $M R R 1^{\mathrm{P} 683 \mathrm{~S}}$ & SC5314 & $\begin{array}{c}M R R 1^{P 683 S}:: \mathrm{FRT} / M R R 1^{P 683 S}:: \mathrm{FRT} \\
U P C 2-1 / U P C 2-2\end{array}$ & Schubert, 2011 \\
\hline$\Delta u p c 2 \mathrm{MRR} 1 \mathrm{R} 34 \mathrm{~A}$ & MRRIupc $2 \Delta / \Delta$ & SCMRR1R34A & $\begin{array}{c}M R R 1^{P 683 S}:: \text { FRT } / M R R 1^{P 683 S}:: \text { FRT } \\
\text { upc2-1 } 1:: \text { FRT/upc2-2 } \Delta:: \text { FRT }\end{array}$ & Schubert, 2011 \\
\hline SCTAC1R34A & $T A C 1^{\mathrm{G} 980 \mathrm{E}}$ & SC5314 & $\begin{array}{c}T A C 1^{G 980 E}:: \mathrm{FRT} / T_{A C 1^{G 980 E}:: \mathrm{FRT}} \\
\text { UPC2-1/UPC2-2 }\end{array}$ & Sasse, 2011 \\
\hline SCupc2TAC1R34A1A14A & TAC1upc $2 \Delta / \Delta$ & SCTAC1R34A & 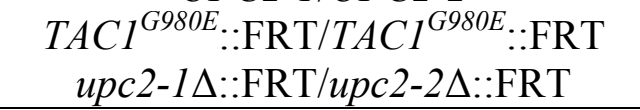 & This study \\
\hline
\end{tabular}


Qiagen; Germantown, MD) and sequenced on an ABI Model 3130XL Genetic Analyzer using the ERG11 sequencing primers (Table 2-2), resulting in full-length sequence from both strands of the CaERG11 gene. The sequencing was performed using six sets of clones derived from three independent PCRs for each strain/isolate sequenced.

Sequenced plasmids of the ERG11 ORF whose predicted translation indicated an amino acid substitution were digested with restriction enzymes ApaI and XhoI, which excised the full-length ORF from the plasmid, and the ERG11 alleles were cloned upstream of the $S A T 1$-flipper cassette into the $A p a \mathrm{I}$ and XhoI sites of plasmid pSFS2 (34). The $E R G 11$ downstream segments were amplified with ExTaq (Takara) using primers $E R G 11$-C and ERG11-D and cloned downstream of the $S A T 1$-flipper cassette in pSFS2 using the NotI and SacII sites.

\section{Construction of UPC2 Deletion Strains}

Plasmid pSFS2 contains the entire $S A T 1$-flipper cassette (34). The $S A T 1$-flipper cassette consists of the $S A T 1$ selectable marker which confers resistance to nourseothricin and the $F L P$ flipper recombinase gene both flanked by $F R T$ sites (flipper recombinase target sequences). The UPC2 deletion cassette (pUPC2M2) was developed by Dunkel et al, where the 5' upstream sequence from positions -373 to +15 was cloned downstream of the $S A T 1$-flipper cassette while the 3' sequence from positions +2097 to +2437 was cloned upstream of the $S A T 1$-flipper cassette (Table 2-2) (216). Upon transformation with the gel-purified SacI-ApaI fragment from pUPC2M2 into the parent strain the SAT1flipper was inserted into the coding region of one allele from positions +16 to +2096 , and such positive transformants $\left(\mathrm{Nou}^{\mathrm{R}}\right)$ were selected on YPD-nourseothricin agar plates containing $200 \mu \mathrm{g} / \mathrm{ml}$ of nourseothricin. Induction of the FLP gene occurred by growing the transformants in YPD medium for 24 hours without selective pressure. Positive cells $\left(\mathrm{Nou}^{\mathrm{S}}\right)$ were selected by replica plating onto YPD plates with and without $200 \mu \mathrm{g} / \mathrm{ml}$ of nourseothricin. Upon induction of the FLP gene, the cassette was excised such that only one copy of the $F R T$ site remains in the locus. Another round was required to disrupt the second allele. Appropriate gene disruption and complementation for two independent strains was confirmed by Southern hybridization (34).

\section{Isolation of Genomic DNA and Southern Hybridization}

Genomic DNA was isolated as described previously (217). Four $\mu \mathrm{g}$ of DNA were digested with an appropriate restriction endonuclease, separated on a 1\% agarose gel, and after staining with ethidium bromide, were transferred by vacuum blotting onto a nylon membrane and fixed by UV cross-linking. Southern hybridization with enhanced chemiluminescence-labeled probes was performed with the Amersham ECL Direct nucleic acid labeling and detection system according to the instructions of the manufacturer. 
Table 2-2. Primers used in this study

\begin{tabular}{|c|c|}
\hline Primer & Sequence \\
\hline \multicolumn{2}{|l|}{ qRT-PCR } \\
\hline$A C T 1-\mathrm{F}$ & 5'-ACGGTGAAGAAGTTGCTGCTTTAGTT-3' \\
\hline$A C T 1-\mathrm{R}$ & 5'-CGTCGTCACCGGCAAAA-3' \\
\hline$B M R I-\mathrm{F}$ & 5'-ACATAAATACTTTGCCCATCCAGAA-3' \\
\hline$B M R I-\mathrm{R}$ & 5'-AAGAGTTGGTTTGTAATCGGCTAAA-3' \\
\hline$C D R 1-\mathrm{F}$ & 5'-ATTCTAAGATGTCGTCGCAAGATG-3' \\
\hline CDRI-R & 5'-AGTTCTGGCTAAATTCGTAATGTTTTC-3' \\
\hline$C D R 2-\mathrm{F}$ & 5'-TAGTCCATTCAACGGCAACATT-3' \\
\hline CDR2-R & 5'-CACCCAGTATTTGGCATTGAAA-3' \\
\hline CFL4-F & 5'-GCAATGGTTGACAGGTTGGAA-3' \\
\hline CFL4-R & 5'-GCAATGTGACGATGATAAGTGACAA-3' \\
\hline ERG11-F & 5'-CССCTATTAATTTTGTTTTCCCTAATTTAC-3' \\
\hline ERG11-R & 5'-CACGTTCTCTTCTCAGTTTAATTTCTTTC-3' \\
\hline FET3-F & 5'-GCCGGTGTCTTAGGTTTAGCC-3' \\
\hline FET3-R & 5'-CTAGCAACTCTTTCTTCAACATCGG-3' \\
\hline$F R P 1-\mathrm{F}$ & 5'-CTTCCAATACCATCCATTCACGAT-3' \\
\hline$F R P 1-\mathrm{R}$ & 5'-АТCTCСССАCTTTCAGCAAGAC-3' \\
\hline FTR1-F & 5'-ATTGTTGTTTCAGTGCTTTTGGC-3' \\
\hline FTR1-R & 5'-GGTCGGAACTACCACCCATAGA-3' \\
\hline \multicolumn{2}{|l|}{ ERG11 cloning } \\
\hline ERG11-F & 5'-GGGCCCTTTGAGAACAGCCACACGACAAC-3' \\
\hline ERG11-R & 5'-CTCGAGGACAAAAACCATCAACCGATTCCG-3' \\
\hline \multicolumn{2}{|c|}{ ERG11 sequencing } \\
\hline$E R G 11 \mathrm{seq} \mathrm{B}$ & 5'-TATTTTCACTGCTTCAAGATCT-3' \\
\hline$E R G 11 \mathrm{seq} \mathrm{C}$ & 5'-CCAAAAGGTCATTATGTTTTAG-3' \\
\hline$E R G 11 \mathrm{seq} \mathrm{E}$ & 5'-AATGAGGTTTTTCACCTAAATG-3' \\
\hline$E R G 11$ seq $\mathrm{F}$ & 5'-CCСTTTACCGAAAACTGGAGTA-3' \\
\hline $\mathrm{T} 7$ & 5'-TAATACGACTCACTATAGGG-3' \\
\hline M13R & 5'-CAGGAAACAGCTATGACC-3' \\
\hline \multicolumn{2}{|c|}{ UPC2 mutant construction } \\
\hline$U P C 2-\mathrm{A}$ & 5'-GGGCCCGAGATCTTGATGTCATTAG-3' \\
\hline$U P C 2-\mathrm{B}$ & 5'-CTCGAGCTATATCTTCAATGAACTG-3' \\
\hline$U P C 2-\mathrm{C}$ & 5'-CCGCGGACAGGTCAATACCGCGTAG-3' \\
\hline$U P C 2-\mathrm{D}$ & 5'-GAGCTCGTTCCTCTAGTATCACTCTT-3' \\
\hline
\end{tabular}

Underlined sequence reflects the introduction of a restriction site sequence. 


\section{RNA Isolation for qRT-PCR}

RNA was isolated using a small-scale hot phenol method of RNA isolation described by Schmitt et al (218). Briefly, overnight cultures were diluted to an $\mathrm{OD}_{600}$ of 0.2 in $20 \mathrm{ml}$ YPD and then incubated at $30^{\circ} \mathrm{C}$ with shaking for 3 hours. Cells were collected by centrifugation, and stored at $-80^{\circ} \mathrm{C}$. Cell pellets were resuspended in $950 \mu \mathrm{l}$ of AE buffer and then transferred to a 2-ml RNAse-free microcentrifuge tube containing $950 \mu \mathrm{l}$ acid phenol ( $\mathrm{pH} 4.3$ ) with $1 \%$ SDS. Cells were incubated at $65^{\circ} \mathrm{C}$ for 10 minutes then lysates were clarified by centrifugation. The supernatant was then divided into two new 2-ml microcentrifuge tubes containing $950 \mu 1$ of chloroform and mixed. The sample was then subjected to centrifugation again, and the top aqueous layer was transferred to a new tube containing $1 \mathrm{ml}$ of isopropanol and $100 \mu 12 \mathrm{M}$ sodium acetate. The RNA pellet was subsequently washed with $500 \mu 1$ of $70 \%$ ethanol and collected by centrifugation. The RNA pellet was resuspended in DNase/RNase-free $\mathrm{H}_{2} \mathrm{O}$. Quantity and purity were determined spectrophotometrically at absorbances of $\mathrm{A}_{260}$ and $\mathrm{A}_{280}$.

\section{Quantitative RT-PCR}

First-strand cDNAs were synthesized from $1 \mu \mathrm{g}$ of total RNA using SuperScript first-strand synthesis system for RT-PCR (Invitrogen). Gene-specific primers

(Table 2-2) were designed using Primer Express software (Applied Biosystems) and synthesized by Integrated DNA Technologies (Coralville, IA). Quantitative PCRs were performed in triplicate using the 7000 sequence detection system (Applied Biosystems), independently amplifying $A C T 1$ (normalizing gene) and the genes of interest (GOI) as described previously (219).

\section{RNA Isolation for Microarray}

RNA was isolated using a large-scale version of the hot phenol method of RNA isolation described by Schmitt et al. (218). Briefly, overnight cultures were diluted to an $\mathrm{OD}_{600}$ of 0.005 in $100 \mathrm{ml} \mathrm{YPD}$ and then incubated at $30^{\circ} \mathrm{C}$ with shaking for an additional 8 hours to an $\mathrm{OD}_{600}$ of 1.0. Cultures were diluted again to an $\mathrm{OD}_{600}$ of 0.025 in $100 \mathrm{ml}$ fresh YPD, allowed to incubate at $30^{\circ} \mathrm{C}$ with shaking for one doubling, inoculated with or without $10 \mu \mathrm{g} / \mathrm{ml} \mathrm{FLC}$, and then incubated at $30^{\circ} \mathrm{C}$ with shaking for 6 hours. Cells were collected by centrifugation, and stored at $-80^{\circ} \mathrm{C}$. Cell pellets were resuspended in $12 \mathrm{ml}$ of AE buffer and then transferred to 50-ml Oak Ridge tubes treated with RNAse Away (Molecular BioProducts) containing $12 \mathrm{ml}$ acid phenol (pH 4.3) with 1\% SDS. Cells were incubated at $65^{\circ} \mathrm{C}$ for 10 minutes then lysates were clarified by centrifugation. The supernatant was then transferred to a new tube containing $15 \mathrm{ml}$ of chloroform and mixed. The sample was then subjected to centrifugation again, and the top aqueous layer was transferred to a new tube containing one volume of isopropanol and 0.1 volume $2 \mathrm{M}$ sodium acetate. The RNA pellet was subsequently washed with $10 \mathrm{ml}$ of $70 \%$ ethanol and collected by centrifugation. The RNA pellet was resuspended in DNase/RNase-free 
$\mathrm{H}_{2} \mathrm{O}$. Quantity and purity were determined spectrophotometrically at absorbances of $\mathrm{A}_{260}$ and $\mathrm{A}_{280}$.

\section{Transcriptional Profiling}

Gene expression profiles were obtained by hybridizing labeled cRNAs generated from C. albicans total RNA onto Affymetrix C. albicans custom expression arrays (CAN07; 49-5241) (216), which have been described previously (201). Microarray hybridization and analysis were performed as described previously (201). Genes were considered to be differentially expressed in response to drug if their expression changed by $\geq 1.5$-fold in two independent experiments. Genes induced by FLC were considered to be $U P C 2$-dependent if the induction was abrogated in the deletion mutant was $\geq 2.0$-fold $(50 \%)$ less than the wild-type. All microarray data is available for download from the NCBI at $G E O$ (Accession number: pending).

\section{RESULTS}

\section{Disruption of UPC2 Results in Enhanced Fluconazole Activity}

In order to further investigate the requirement of $U P C 2$ for susceptibility to fluconazole, we subjected the upc $2 \Delta / \Delta$ mutant derived from azole-susceptible isolate SC5314 to various azole susceptibility tests examining both MIC and MFC using nutrient rich YPD media in order to detect strong phenotypes despite existing in an environment that promotes growth. Consistent with previous observations, disruption of UPC2 resulted in a marked reduction in fluconazole MIC by broth microdilution (Table 2-3), Etest (Figure 2-1A), $72 \mathrm{hr}$ regrowth (Figure 2-1B), and spot assay (Figure 2-1C). Interestingly at $24 \mathrm{hr}$ in YPD, the fluconazole MFC for SC5314 was $>64 \mu \mathrm{g} / \mathrm{ml}$ whereas for the upc $2 \Delta / \Delta$ mutant it was $0.25 \mu \mathrm{g} / \mathrm{ml}$ (Table 2-3). At $48 \mathrm{hr}$ the MIC, as measured by Etest, for SC5314 was $1.0 \mu \mathrm{g} / \mathrm{ml}$ and a halo of reduced growth (but not a clear zone of inhibition) was observed up to the Etest strip consistent with the fungistatic nature of fluconazole. The $48 \mathrm{hr}$ MIC by Etest for the upc $2 \Delta / \Delta$ mutant was $0.032 \mu \mathrm{g} / \mathrm{ml}$ and a clear zone of inhibition around the Etest strip was observed (Figure 2-1A). We also used a $72 \mathrm{hr}$ endpoint for a broth microdilution assay in YPD as a way to assess the ability of the organism to resume growth in the presence of fluconazole. SC5314 was able to resume growth in all concentrations of fluconazole tested whereas the upc $2 \Delta / \Delta$ mutant was not (Table 2-3, Figure 2-1B). When plated on YPD agar plates containing $10 \mu \mathrm{g} / \mathrm{ml}$ fluconazole, growth was reduced in the presence of fluconazole for the upc $2 \Delta / \Delta$ mutant as compared to SC5314 (Figure 2-1C). Time-kill analysis showed increased fungistatic activity of $10 \mu \mathrm{g} / \mathrm{ml}$ fluconazole against the $u p c 2 \Delta / \Delta$ mutant as compared to its parent strain (Figure 2-1D). All phenotypes were reverted by reintegration of one allele of the disrupted gene. 
Table 2-3. MICs and MFCs in YPD in SC5314 background

\begin{tabular}{|c|c|c|c|c|c|c|c|}
\hline \multirow[b]{2}{*}{ Strain } & \multirow{2}{*}{$\begin{array}{c}\text { Relevant Characteristics or } \\
\text { Genotype }\end{array}$} & \multicolumn{3}{|c|}{$\operatorname{MIC}(\mu \mathrm{g} / \mathrm{ml})$} & \multicolumn{3}{|c|}{$\operatorname{MFC}(\mu \mathrm{g} / \mathrm{ml})$} \\
\hline & & $24 \mathrm{hr}$ & $48 \mathrm{hr}$ & $72 \mathrm{hr}$ & $24 \mathrm{hr}$ & $48 \mathrm{hr}$ & $72 \mathrm{hr}$ \\
\hline SC5314 & $U P C 2-1 / U P C 2-2$ & 0.5 & 0.5 & $>64$ & $>64$ & $>64$ & $>64$ \\
\hline upc $2 \Delta / \Delta$ & upc2-1 $1 \Delta:: \mathrm{FRT} / u p c 2-2 \Delta:: \mathrm{FRT}$ & $\leq 0.125$ & 0.25 & 0.25 & 0.25 & 0.25 & 0.25 \\
\hline upc $2 \Delta / \triangle+U P C 2$ & upc2-1 $\triangle:: \mathrm{FRT} / U P C 2^{S 1-1}-\mathrm{caSAT1}$ & 0.5 & 0.5 & $>64$ & $>64$ & $>64$ & $>64$ \\
\hline
\end{tabular}


Figure 2-1. $\quad$ UPC2 impacts fluconazole susceptibility in SC5314 background (A) Effect of UPC2 on MIC and growth on YPD agar as determined by Etest. A confluent lawn of $C$. albicans was streaked prior to the addition of Etest strips and then incubated for 48 hours. (B) MIC heat map of SC5314, UPC2 mutant, and complimented derivative. Susceptibility was determined by broth microdilution in YPD at 72 hours. Growth was quantified spectrophotometrically and assigned to a colorimetric scale. (C) Effect on UPC2 on the ability to grow on solid media containing fluconazole. From 4fold serial dilutions of $C$. albicans strains, $2 \mu$ l aliquots were spotted onto YPD agar $+/-$ $10 \mu \mathrm{g} / \mathrm{mL}$ FLC and incubated for 48 hours. (D) Effect of fluconazole on UPC2 by timekill assay. SC5314 or upc $2 \Delta / \Delta$ cells were diluted in YPD medium containing fluconazole $(10 \mu \mathrm{g} / \mathrm{mL})$ or the solvent, DMSO. After 0, 6, 12, and 24 hours, samples from each were diluted and plated for colony forming units. 


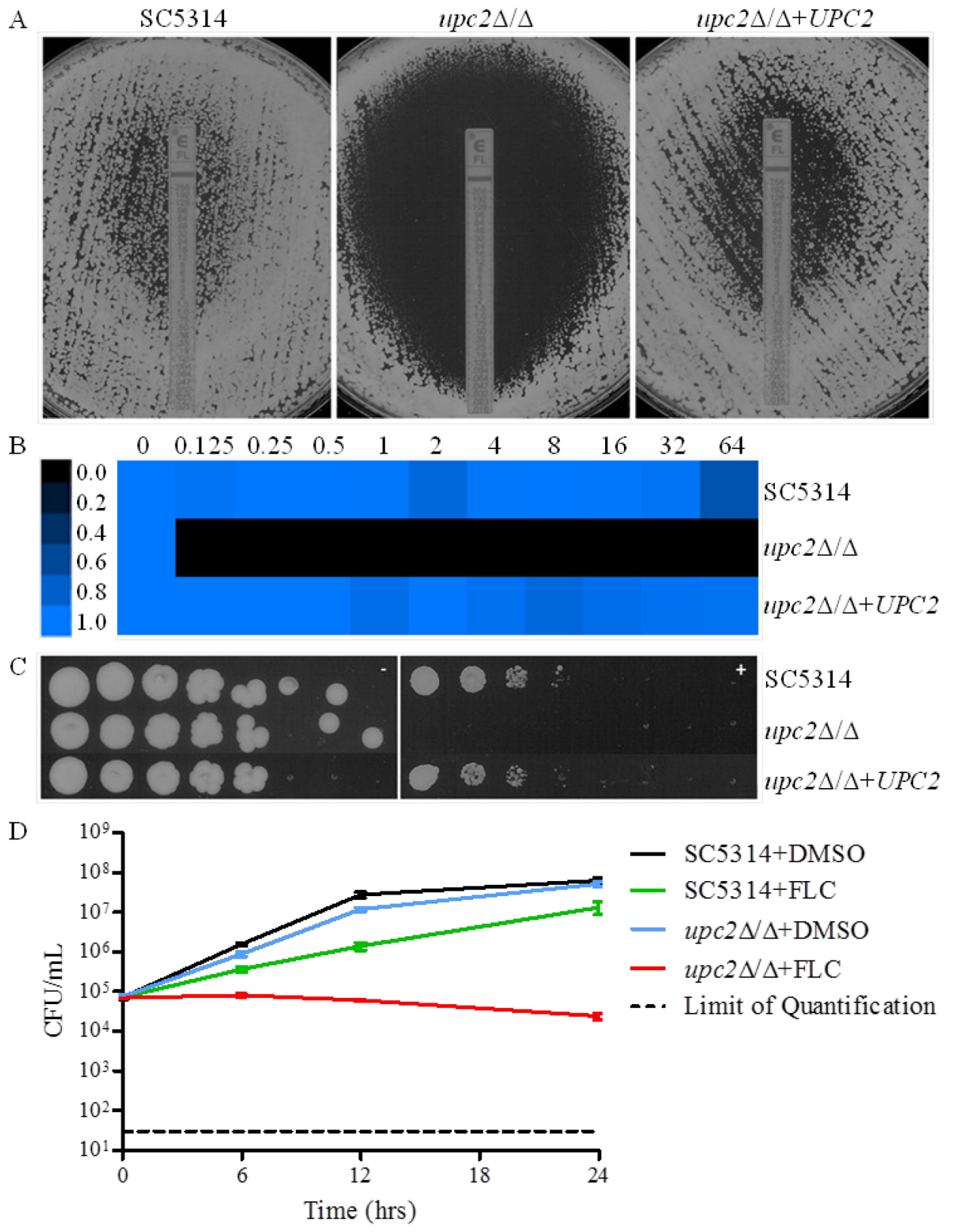




\section{Disruption of UPC2 in Strains Containing Resistance Mutations in MRR1, TAC1, and ERG11 Also Enhances Fluconazole Activity}

In order to investigate the requirement for $U P C 2$ in the setting of specific mechanisms of azole resistance, independent mutants were constructed in strains containing two copies of a gene conferring reduced susceptibility to fluconazole: $M R R 1^{\mathrm{P} 683 \mathrm{~S}}, T A C 1^{\mathrm{G} 980 \mathrm{E}}$, or $E R G 11^{\mathrm{K} 143 \mathrm{R}}$. The $M R R 1^{\mathrm{P} 683 \mathrm{~S}}$ and $T A C 1^{\mathrm{G} 980 \mathrm{E}}$ alleles contain gain-of-function mutations which render the transcription factors they encode constitutively active, resulting in upregulation of either MDR1 or $C D R 1$ and $C D R 2$, respectively, and decreased fluconazole susceptibility $(195,196,220,221)$. The $E R G 11^{\mathrm{K} 143 \mathrm{R}}$ allele contains a point mutation postulated to be located near the azole access channel, interfering with entry of fluconazole, resulting in decreased fluconazole susceptibility (222). Again the disruption of UPC2 in each background resulted in a marked reduction in MIC and MFC by all methods and the trend in susceptibility seen for MRRIupc $2 \Delta / \Delta$ was consistent with what we have previously observed (214). The MFCs at $24 \mathrm{hr}$ in YPD for ERG1 lupc $2 \Delta / \Delta, M R R 1$ upc $2 \Delta / \Delta$ and TAClupc $2 \Delta / \Delta$ were all reduced from $8 \mu \mathrm{g} / \mathrm{ml},>64 \mu \mathrm{g} / \mathrm{ml}$, and $>64 \mu \mathrm{g} / \mathrm{ml}$ in their background strains to $1 \mu \mathrm{g} / \mathrm{ml}, 4 \mu \mathrm{g} / \mathrm{ml}$, and $2 \mu \mathrm{g} / \mathrm{ml}$ respectively (Table 2-4). At $48 \mathrm{hr}$ the MICs by Etest for ERG1 lupc $2 \Delta / \Delta$, MRRlupc $2 \Delta / \Delta$ and TAClupc $2 \Delta / \Delta$ were all reduced from $1.5 \mu \mathrm{g} / \mathrm{ml}, 4 \mu \mathrm{g} / \mathrm{ml}$, and $8 \mu \mathrm{g} / \mathrm{ml}$ in their background strains to $0.19 \mu \mathrm{g} / \mathrm{ml}, 0.5 \mu \mathrm{g} / \mathrm{ml}$, and $0.5 \mu \mathrm{g} / \mathrm{ml}$ respectively (Figure 2-2A). Growth was also reduced when plated on YPD agar plates containing fluconazole $10 \mu \mathrm{g} / \mathrm{ml}$ for the MRRIupc $2 \Delta / \Delta$ and TAClupc $2 \Delta / \Delta$ strains as compared to their background strain (Figure 2-2B). Consistent with their fluconazole MICs, both the $E R G 11^{\mathrm{K} 143 \mathrm{R}}$ strain and its upc $2 \Delta / \Delta$ derivative were unable to grow at this concentration of fluconazole.

\section{Disruption of Upc2 Overrides Clinical Drug Resistance}

Since disruption of $U P C 2$ in strains containing one resistance mechanism resulted in enhanced fluconazole activity, we wanted to further examine the extent to which disruption of UPC2 influences high-level azole resistance in the presence of multiple resistance mechanisms. We constructed upc $2 \Delta / \Delta$ mutant strains in the background of an azole-resistant clinical isolate (12-99) known to carry four of the most common mechanisms of azole resistance: overexpression of $C D R 1$ and $C D R 2$, overexpression of $M D R 1$, overexpression of $E R G 11$, and mutation in ERG11 (223). As was observed for isolate SC5314 and the isogenic resistant strains, the disruption of UPC2 resulted in a marked reduction in MIC and MFC by all methods. The MFC at $24 \mathrm{hr}$ in YPD for 12-99 was $>64 \mu \mathrm{g} / \mathrm{ml}$ whereas for $12-99 u p c 2 \Delta / \Delta$ was $16 \mu \mathrm{g} / \mathrm{ml}$ (Table 2-5). The fluconazole MIC by Etest at $48 \mathrm{hr}$ was $>256 \mu \mathrm{g} / \mathrm{ml}$ and confluent growth was observed for 12-99, whereas an MIC of $1.5 \mu \mathrm{g} / \mathrm{ml}$ and a clear zone of inhibition was observed for 1299 upc $2 \Delta / \Delta$ (Figure 2-3A). Likewise, in broth microdilution assays after $72 \mathrm{hr}$ the parent strain was able to grow in all concentrations of fluconazole tested whereas 12-99upc $2 \Delta / \Delta$ grew less well (Figure 2-3B). Growth was also reduced when plated on YPD agar plates containing fluconazole $10 \mu \mathrm{g} / \mathrm{ml}$ for $12-99 u p c 2 \Delta / \Delta$ as compared to its parent strain

(Figure 2-3C). As was observed in the SC5314 background, time-kill analysis also 
Table 2-4. MICs and MFCs in YPD in background of strains expressing resistance mechanisms

\begin{tabular}{|c|c|c|c|c|c|c|c|}
\hline \multirow[b]{2}{*}{ Strain } & \multirow{2}{*}{$\begin{array}{c}\text { Relevant Characteristics or } \\
\text { Genotype }\end{array}$} & \multicolumn{3}{|c|}{$\operatorname{MIC}(\mu \mathrm{g} / \mathrm{ml})$} & \multicolumn{3}{|c|}{$\operatorname{MFC}(\mu \mathrm{g} / \mathrm{ml})$} \\
\hline & & $24 \mathrm{hr}$ & $48 \mathrm{hr}$ & $72 \mathrm{hr}$ & $24 \mathrm{hr}$ & $48 \mathrm{hr}$ & $72 \mathrm{hr}$ \\
\hline SC5314 & $U P C 2-1 / U P C 2-2$ & 0.5 & 0.5 & $>64$ & $>64$ & $>64$ & $>64$ \\
\hline$E R G 11^{\mathrm{K} 143 \mathrm{R}}$ & $\begin{array}{c}E R G 11^{K 143 R}:: \mathrm{FRT} / E R G 11^{K 143 R}:: \mathrm{FRT} \\
U P C 2-1 / U P C 2-2\end{array}$ & 4 & 8 & 8 & 8 & 8 & 32 \\
\hline$E R G 11$ upc $2 \Delta / \Delta$ & 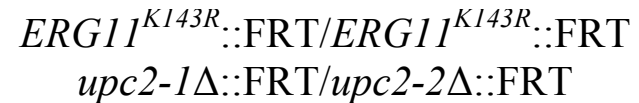 & 0.5 & 1 & 1 & 1 & 1 & 1 \\
\hline$M R R 1^{\mathrm{P} 683 \mathrm{~S}}$ & $\begin{array}{c}M R R 1^{P 683 S}:: \mathrm{FRT} / M R R 1^{P 683 S}:: \mathrm{FRT} \\
U P C 2-1 / U P C 2-2\end{array}$ & 16 & $>64$ & $>64$ & $>64$ & $>64$ & $>64$ \\
\hline MRRIupc $2 \Delta / \Delta$ & 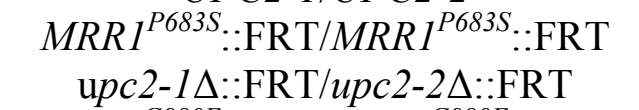 & 2 & 2 & 4 & 4 & 4 & 4 \\
\hline$T A C 1^{\mathrm{G} 980 \mathrm{E}}$ & $\begin{array}{c}T A C 1^{G 980 E}:: \mathrm{FRT} / T A C 1^{G 980 E}:: \mathrm{FRT} \\
\text { UPC2-1/UPC2-2 }\end{array}$ & 16 & $>64$ & $>64$ & $>64$ & $>64$ & $>64$ \\
\hline$T A C 1$ upc $2 \Delta / \Delta$ & 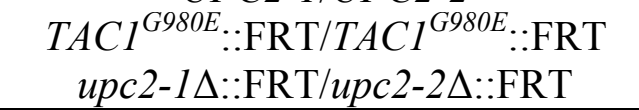 & 2 & 2 & 2 & 2 & 2 & 2 \\
\hline
\end{tabular}



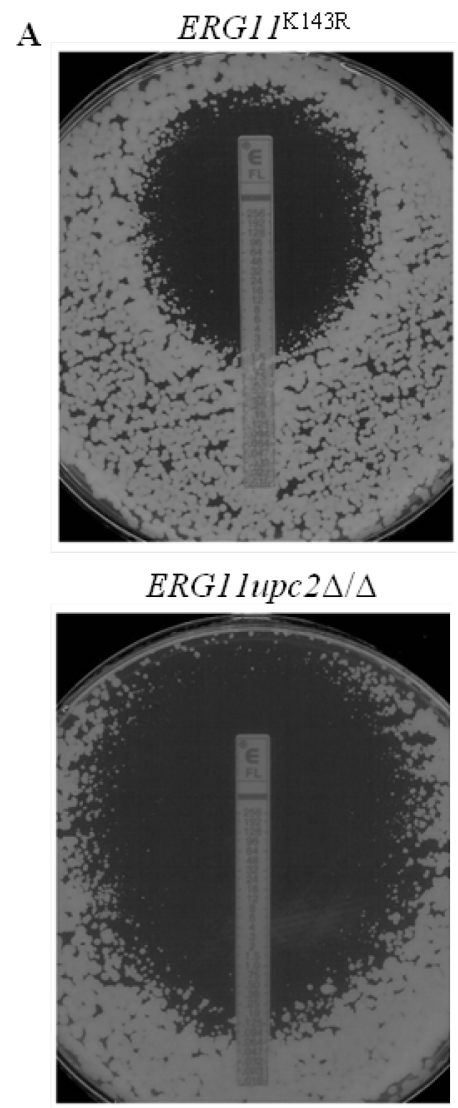

B

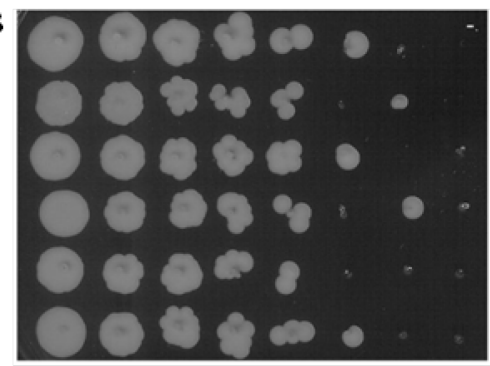

MRRI $I^{\mathrm{P} 683 \mathrm{~S}}$

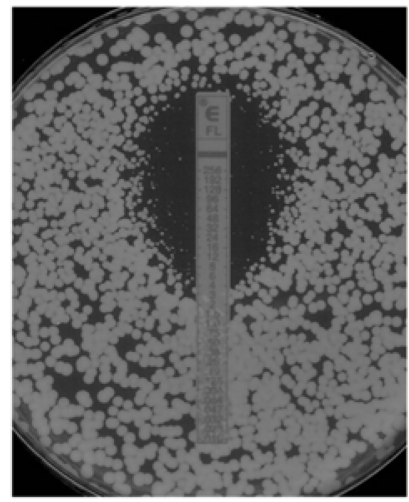

MRRIupc $2 \Delta / \Delta$
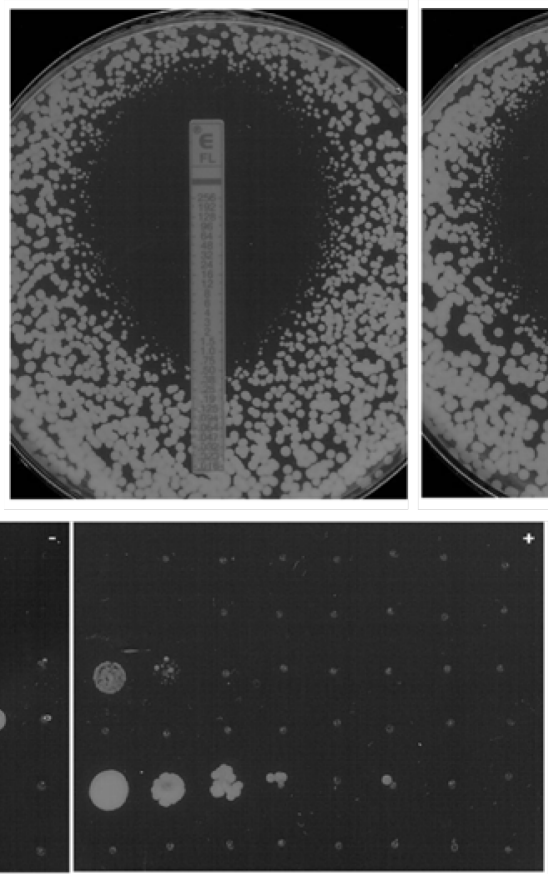

$T A C l^{\mathrm{G} 980 \mathrm{E}}$

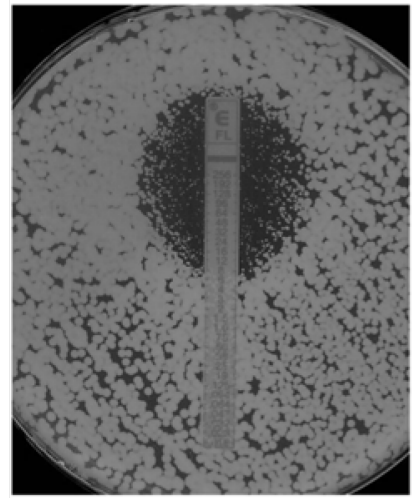

$T A C 1 u p c 2 \Delta / \Delta$

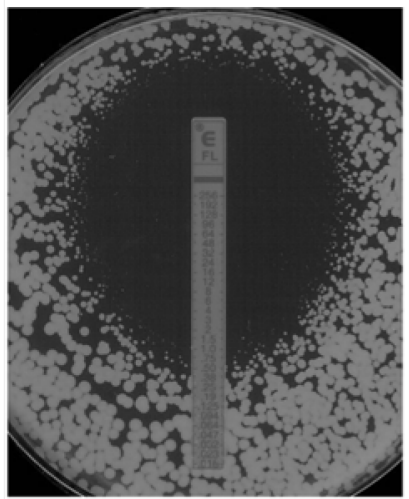

ERGI I $143 \mathrm{R}$

ERG1 1upc $2 \Delta / \Delta$

MRRI $1^{\mathrm{P} 683 \mathrm{~S}}$

MRRIupc $2 \Delta / \Delta$

TAC ${ }^{\mathrm{G} 980 \mathrm{E}}$

TAClupc $2 \Delta / \Delta$

Figure 2-2. UPC2 impacts fluconazole susceptibility in background of strains expressing resistance mechanisms

(A) Effect of UPC2 in resistant backgrounds on MIC and growth on YPD agar as determined by Etest. A confluent lawn of C. albicans was streaked prior to the addition of Etest strips and then incubated for 48 hours. (B) Effect of UPC2 in resistance backgrounds on the ability to grow on solid media containing fluconazole. From 4-fold serial dilutions of $C$. albicans strains, $2 \mu$ l aliquots were spotted onto YPD agar $+/-10$ $\mu \mathrm{g} / \mathrm{mL}$ FLC and incubated for 48 hours. 
Table 2-5. MICs and MFCs in YPD of strains in 12-99 background

\begin{tabular}{|c|c|c|c|c|c|c|c|}
\hline \multirow[b]{2}{*}{ Strain } & \multirow{2}{*}{$\begin{array}{c}\text { Relevant Characteristics } \\
\text { or Genotype }\end{array}$} & \multicolumn{3}{|c|}{$\mathrm{MIC}(\mu \mathrm{g} / \mathrm{ml})$} & \multicolumn{3}{|c|}{$\operatorname{MFC}(\mu \mathrm{g} / \mathrm{ml})$} \\
\hline & & $24 \mathrm{hr}$ & $48 \mathrm{hr}$ & $72 \mathrm{hr}$ & $24 \mathrm{hr}$ & $48 \mathrm{hr}$ & $72 \mathrm{hr}$ \\
\hline SC5314 & $U P C 2-1 / U P C 2-2$ & 0.5 & 0.5 & $>64$ & $>64$ & $>64$ & $>64$ \\
\hline $2-79$ & Susceptible isolate & 1 & 1 & $>64$ & $>64$ & $>64$ & $>64$ \\
\hline $12-99$ & Resistant isolate & $>64$ & $>64$ & $>64$ & $>64$ & $>64$ & $>64$ \\
\hline $12-99 u p c 2 \Delta / \Delta$ & upc $2 \Delta::$ FRT/upc $2 \Delta::$ FRT & 4 & 4 & 8 & 16 & 16 & 16 \\
\hline
\end{tabular}


Figure 2-3. UPC2 impacts fluconazole susceptibility in 12-99 background

(A) Effect of UPC2 in 12-99 on MIC and growth on YPD agar as determined by Etest. A confluent lawn of $C$. albicans was streaked prior to the addition of Etest strips and then incubated for 48 hours. (B) MIC heat map of 2-79, 12-99, and UPC2 mutant. Susceptibility was determined by broth microdilution in YPD at 72 hours. Growth was quantified spectrophotometrically and assigned to a colorimetric scale. (C) Effect of $U P C 2$ in 12-99 on the ability to grow on solid media containing fluconazole. From 4fold serial dilutions of $C$. albicans strains, $2 \mu$ l aliquots were spotted onto YPD agar +/$10 \mu \mathrm{g} / \mathrm{mL}$ FLC and incubated for 48 hours. (D) Effect of fluconazole on UPC2 in 12-99 by time-kill assay. $12-99$ or 12-99upc $2 \Delta / \Delta$ cells were diluted in YPD medium containing fluconazole $(10 \mu \mathrm{g} / \mathrm{mL})$ or the solvent, DMSO. After $0,6,12$, and 24 hours, samples from each were diluted and plated for colony forming units. 
A
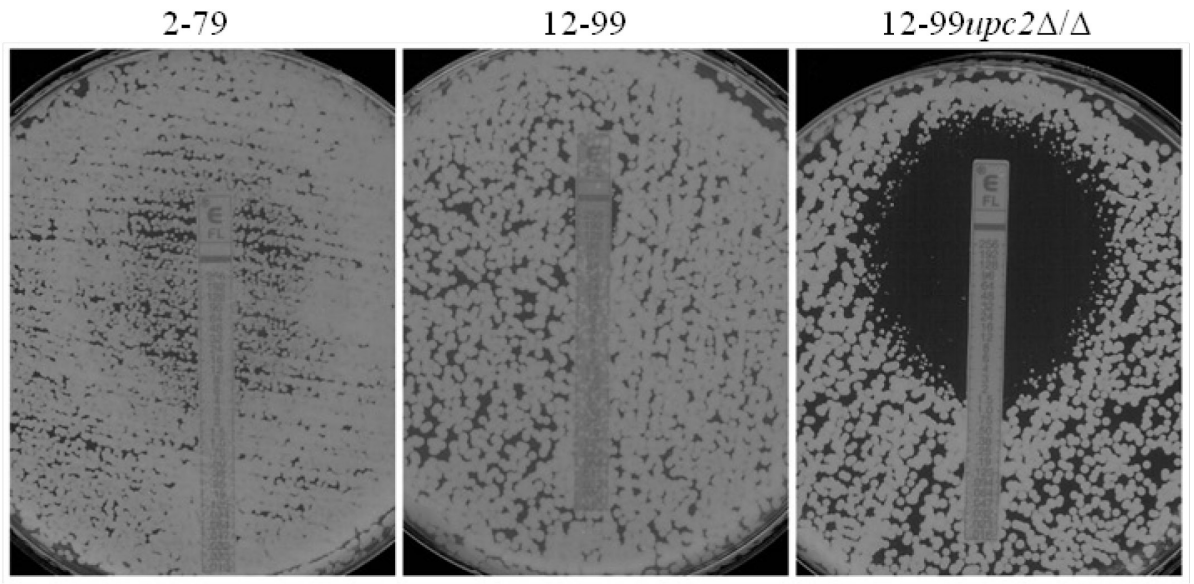

B

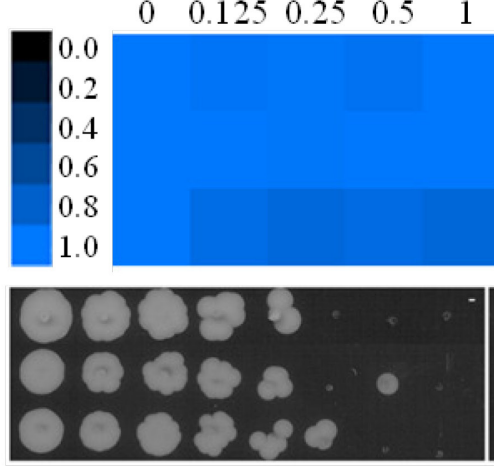

$\begin{array}{llllll}2 & 4 & 8 & 16 & 32 & 64\end{array}$
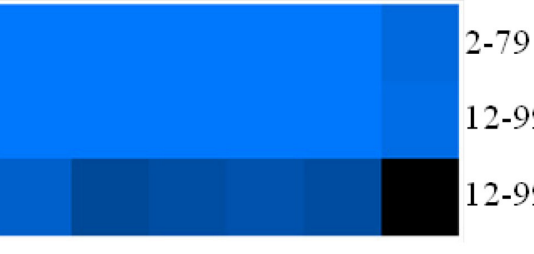

12-99

$12-99 u p c 2 \Delta / \Delta$

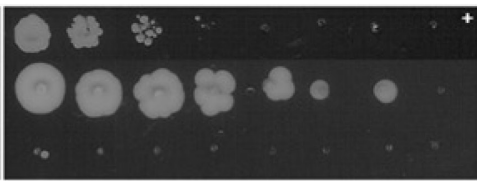

2-79

12-99

$12-99 u p c 2 \Delta / \Delta$
D

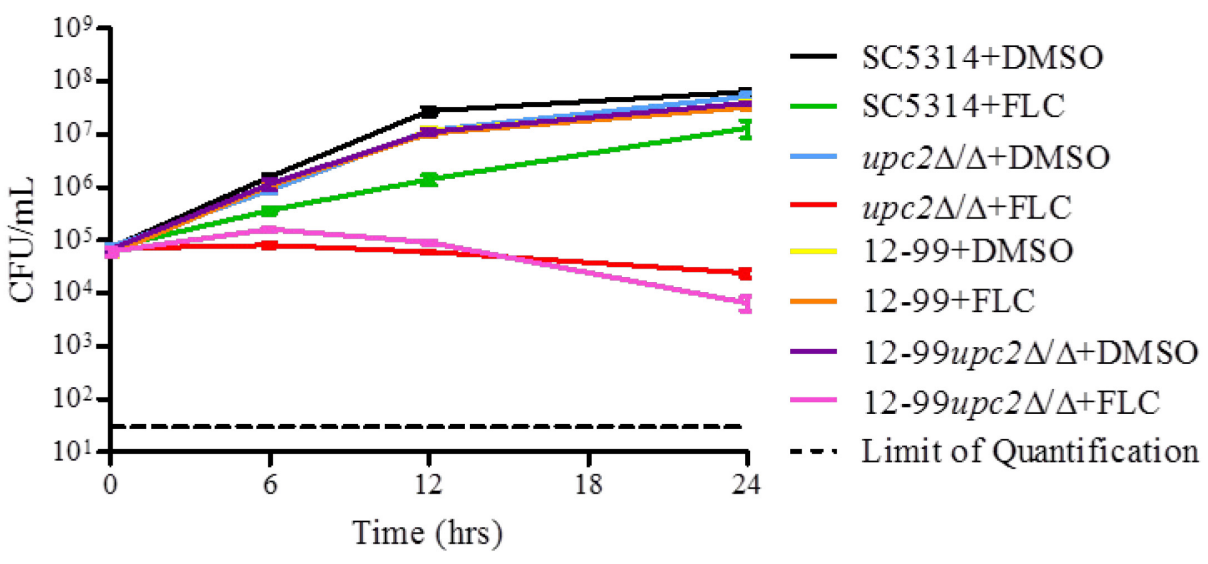


revealed an increased fungistatic effect for fluconazole at $10 \mu \mathrm{g} / \mathrm{ml}$ against $12-99 u p c 2 \Delta / \Delta$ (Figure 2-3D).

\section{Expression of ERG11, CDR1, CDR2, and MDR1 When UPC2 Is Disrupted in Resistant Backgrounds}

In order to determine if the enhanced fluconazole activity was due to decreased expression $E R G 11$ or genes encoding efflux pumps, we measured $E R G 11, C D R 1, C D R 2$, and $M D R 1 \mathrm{mRNA}$ abundance by qRT-PCR in the strains containing a single resistance mechanism, isolate 12-99 and their respective $u p c 2 \Delta / \Delta$ mutants (Figure 2-4). As expected, the upc2 $\Delta / \Delta$ mutant constructed in the SC5314 background showed a reduction in baseline ERG11 expression relative to its parent strain. This was also the case for the upc $2 \Delta / \Delta$ mutants constructed in the $E R G 11^{\mathrm{K} 143 \mathrm{R}}$ and clinical isolate $12-99$ backgrounds. However, both the $T A C 1^{\mathrm{G} 980 \mathrm{E}}$ and $M R R I^{\mathrm{P} 683 \mathrm{~S}}$ strains exhibited reduced $E R G 11$ expression relative to $\mathrm{SC} 5314$ with no appreciable additional reduction in expression when $U P C 2$ was disrupted. Disruption of $U P C 2$ did not result in decreased expression of $C D R 1, C D R 2$, or $M D R 1$ in any background. Interestingly, disruption of $U P C 2$ in $E R G 1$ lupc $2 \Delta / \Delta$ resulted in an increase in expression of these transporter genes, the significance of which is unclear. These data suggest that the enhanced activity of fluconazole observed in resistant strains lacking $U P C 2$ is not due to changes in transporter gene expression levels, but may be associated with a reduction in $E R G$ gene expression, particularly ERG11.

\section{Comparison of the Gene Expression Profiles of Wild-Type Strain SC5314 and $u p c 2 \Delta / \Delta$ Exposed to Fluconazole}

In order to identify genes whose expression in response to fluconazole is influenced by Upc2, we compared the transcriptional profiles of SC5314 and its upc $2 \Delta / \Delta$ derivative after treatment with or without $10 \mu \mathrm{g} / \mathrm{ml}$ fluconazole for 6 hours. Genes were considered to be differentially expressed in response to fluconazole if their expression changed by $\geq 1.5$-fold in two independent experiments (Table A-1 and Table A-2). Fluconazole inducible genes were also considered to be $U P C 2$-dependent if their induction was abrogated in the deletion mutant and was $\geq 2.0$-fold (50\%) less than compared to SC5314. Using these criteria, there were 127 genes upregulated by fluconazole whose induction was abrogated in the absence of UPC2 (Table 2-6). The most common biological processes represented by these genes included the lipid metabolic process, iron ion transport and iron homeostasis, transport, response to stress and chemical stimulus, and the oxidation-reduction process.

\section{Validation of Microarray Data by Real Time RT-PCR}

In order to validate the differential expression of genes identified by microarray, we examined the mRNA abundance for 5 genes of interest using the same RNA isolated 

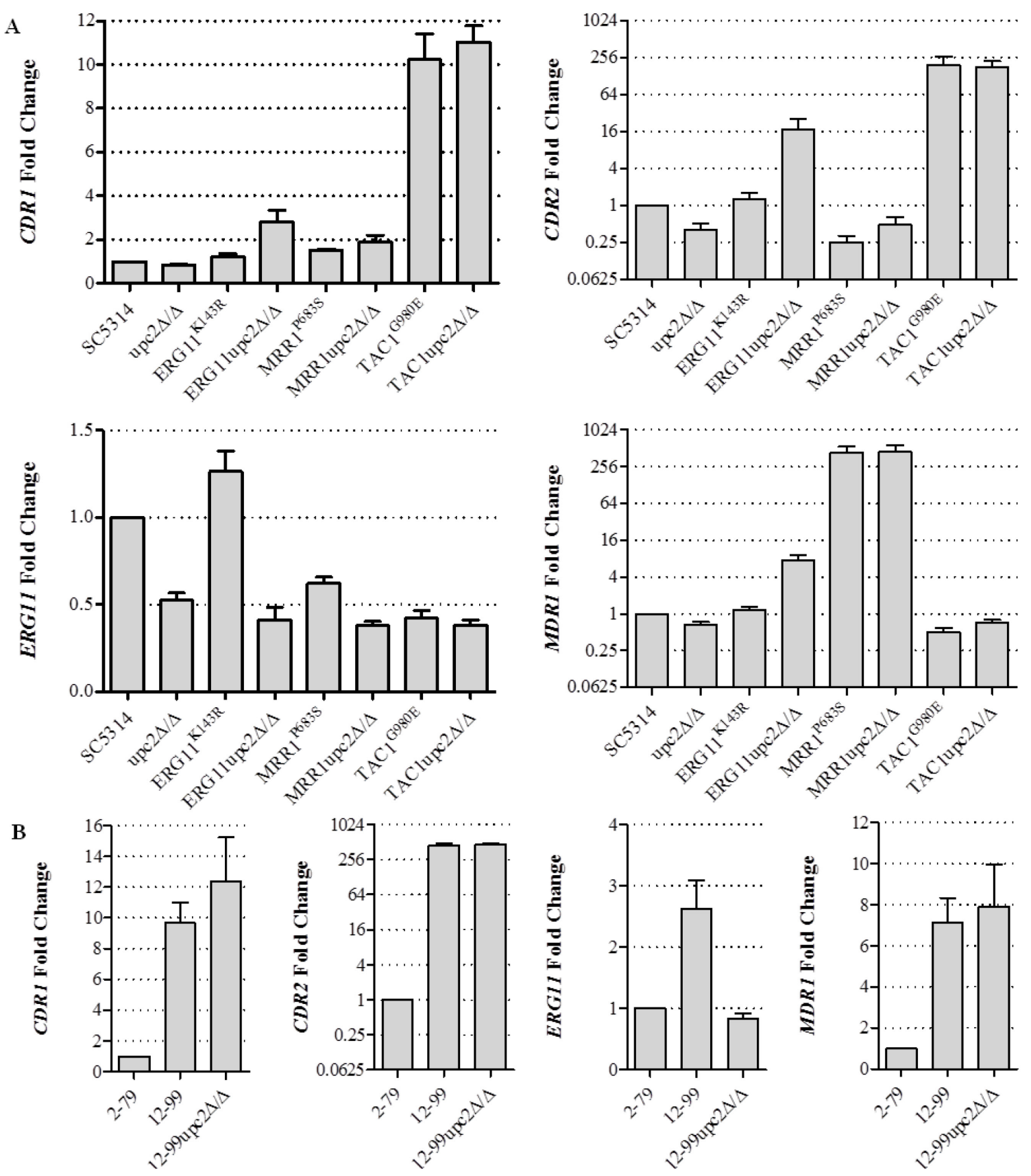

Figure 2-4. Expression of ERG11, CDR1, CDR2, and MDR1 as measured by qRTPCR

Expression levels for $E R G 11, C D R 1, C D R 2$, and $M D R 1$ were measured in triplicate by qRT-PCR and compared to the expression levels in SC5314 (A) and 2-79 (B) in YPD. Error bars represent the standard error of the mean. 
Table 2-6. Genes upregulated by at least 1.5-fold by fluconazole which are dependent upon Upc2

\begin{tabular}{|c|c|c|c|c|c|c|c|c|}
\hline \multirow[b]{3}{*}{ Process $^{a}$} & \multirow[b]{3}{*}{ orf19 Designation } & \multirow[b]{3}{*}{ CGD Name } & \multicolumn{4}{|c|}{ Change in Fold Expression } & & \\
\hline & & & \multicolumn{2}{|c|}{$\begin{array}{c}\text { SC5314+FLC/ } \\
\text { SC5314 (A) } \\
\end{array}$} & \multicolumn{2}{|c|}{$\begin{array}{c}\text { upc } 2 \Delta / \Delta+\mathrm{FLC} / \\
\text { upc } 2 \Delta / \Delta(\mathrm{B})\end{array}$} & \multicolumn{2}{|c|}{ Ratio (B/A) } \\
\hline & & & Exp1 & Exp2 & Exp1 & $\operatorname{Exp2}$ & Exp1 & Exp2 \\
\hline \multirow{7}{*}{$\begin{array}{l}\text { Lipid metabolic } \\
\text { process }\end{array}$} & orf19.1598 & $E R G 24$ & 2.0 & 1.6 & 0.9 & 0.7 & 0.5 & 0.4 \\
\hline & orf19.1631 & ERG6 & 1.8 & 1.7 & 0.4 & 0.5 & 0.2 & 0.3 \\
\hline & orf19.2670 & & 1.6 & 1.8 & 0.4 & 0.7 & 0.2 & 0.4 \\
\hline & orf19.3240 & $E R G 27$ & 2.8 & 2.4 & 1.4 & 1.1 & 0.5 & 0.4 \\
\hline & orf19.4982 & & 2.1 & 2.3 & 0.7 & 0.8 & 0.3 & 0.4 \\
\hline & orf19.7585 & INOI & 9.2 & 20.5 & 0.9 & 0.8 & 0.1 & 0.0 \\
\hline & orf19.922 & ERG11 & 1.6 & 1.5 & 0.8 & 0.7 & 0.5 & 0.4 \\
\hline \multirow[t]{6}{*}{ Iron ion transport } & orf19.1415 & FRE10 & 2.0 & 4.2 & 0.2 & 0.1 & 0.1 & 0.0 \\
\hline & orf19.1932 & CFL4 & 35.6 & 359 & 8.0 & 22 & 0.2 & 0.1 \\
\hline & orf19.4211 & FET3 & 2.8 & 6.2 & 0.7 & 0.2 & 0.3 & 0.0 \\
\hline & orf19.4215 & FET34 & 2.1 & 5.2 & 0.0 & 0.1 & 0.0 & 0.0 \\
\hline & orf19.5634 & $F R P 1$ & 8.9 & 8.2 & 0.1 & 0.2 & 0.0 & 0.0 \\
\hline & orf19.7219 & FTR1 & 3.2 & 7.8 & 0.1 & 0.0 & 0.0 & 0.0 \\
\hline \multirow{4}{*}{$\begin{array}{c}\text { Iron ion } \\
\text { homeostasis }\end{array}$} & orf19.1264 & CFL2 & 2.0 & 6.0 & 0.2 & 0.8 & 0.1 & 0.1 \\
\hline & orf19.1715 & IROI & 4.2 & 8.1 & 2.2 & 1.4 & 0.5 & 0.2 \\
\hline & orf19.5636 & RBT5 & 1.7 & 1.6 & 0.2 & 0.2 & 0.1 & 0.2 \\
\hline & orf19.7114 & $C S A 1$ & 1.7 & 3.2 & 0.7 & 0.6 & 0.4 & 0.2 \\
\hline \multirow[t]{2}{*}{ Transport } & orf19.1352 & TIM22 & 3.6 & 4.4 & 1.3 & 2.0 & 0.4 & 0.4 \\
\hline & orf19.2023 & $H G T 7$ & 20.8 & 21.6 & 9.4 & 7.8 & 0.5 & 0.4 \\
\hline
\end{tabular}


Table 2-6. (Continued)

\begin{tabular}{|c|c|c|c|c|c|c|c|c|}
\hline \multirow[b]{3}{*}{ Process $^{a}$} & \multirow[b]{3}{*}{ orf19 Designation } & \multirow[b]{3}{*}{ CGD Name } & \multicolumn{4}{|c|}{ Change in Fold Expression } & & \\
\hline & & & \multicolumn{2}{|c|}{$\begin{array}{c}\text { SC5314+FLC/ } \\
\text { SC5314 (A) }\end{array}$} & \multicolumn{2}{|c|}{$\begin{array}{c}u p c 2 \Delta / \Delta+F L C / \\
\text { upc } 2 \Delta / \Delta(\mathrm{B})\end{array}$} & \multicolumn{2}{|c|}{ Ratio (B/A) } \\
\hline & & & Exp1 & Exp2 & Exp1 & $\operatorname{Exp2}$ & Exp1 & Exp2 \\
\hline & orf19.2292 & OPT4 & 5.2 & 3.7 & 0.0 & 0.0 & 0.0 & 0.0 \\
\hline & orf19.2350 & & 2.5 & 4.1 & 0.9 & 0.6 & 0.4 & 0.1 \\
\hline & orf19.2785 & ATP7 & 3.1 & 3.1 & 1.3 & 1.5 & 0.4 & 0.5 \\
\hline & orf19.3026 & $M A S 1$ & 1.7 & 1.6 & 0.9 & 0.8 & 0.5 & 0.5 \\
\hline & orf19.3195 & $H I P 1$ & 1.5 & 2.6 & 0.7 & 0.8 & 0.5 & 0.3 \\
\hline & orf19.3232 & & 24.6 & 5.3 & 1.6 & 1.6 & 0.1 & 0.3 \\
\hline & orf19.3668 & $H G T 2$ & 48.3 & 35.5 & 20.1 & 12.3 & 0.4 & 0.3 \\
\hline & orf19.3746 & $I F C 1$ & 2.3 & 2.7 & 0.2 & 0.0 & 0.1 & 0.0 \\
\hline & orf19.4335 & $T N A 1$ & 194.9 & 29.8 & 0.4 & 0.2 & 0.0 & 0.0 \\
\hline & orf19.4384 & HXT5 & 70.6 & 75.5 & 16.9 & 9.7 & 0.2 & 0.1 \\
\hline & orf19.4682 & $H G T 17$ & 45.0 & 24.7 & 4.2 & 4.5 & 0.1 & 0.2 \\
\hline & orf19.4690 & & 16.3 & 20.2 & 1.0 & 1.4 & 0.1 & 0.1 \\
\hline & orf19.5307 & JEN2 & 10.3 & 2.7 & 0.4 & 1.3 & 0.0 & 0.5 \\
\hline & orf19.5753 & HGT10 & 20.6 & 1.6 & 2.6 & 0.8 & 0.1 & 0.5 \\
\hline & orf19.6148 & & 4.4 & 47.3 & 2.0 & 13.8 & 0.4 & 0.3 \\
\hline & orf19.6249 & $H A K 1$ & 5.4 & 5.8 & 1.4 & 1.7 & 0.3 & 0.3 \\
\hline & orf19.6993 & $G A P 2$ & 30.6 & 10.6 & 6.0 & 3.0 & 0.2 & 0.3 \\
\hline & orf19.7093 & $H G T 13$ & 40.9 & 18.8 & 9.8 & 2.6 & 0.2 & 0.1 \\
\hline \multirow[t]{3}{*}{ Response to stress } & orf19.1434 & & 1.7 & 2.7 & 0.8 & 0.9 & 0.5 & 0.3 \\
\hline & orf19.3239 & CTF18 & 2.7 & 2.4 & 1.4 & 0.8 & 0.5 & 0.4 \\
\hline & orf19.3672 & GAL10 & 6.0 & 7.3 & 2.7 & 3.8 & 0.5 & 0.5 \\
\hline
\end{tabular}


Table 2-6. (Continued)

\begin{tabular}{|c|c|c|c|c|c|c|c|c|}
\hline \multirow[b]{3}{*}{ Process $^{a}$} & \multirow[b]{3}{*}{ orf19 Designation } & \multirow[b]{3}{*}{ CGD Name } & \multicolumn{4}{|c|}{ Change in Fold Expression } & & \\
\hline & & & \multicolumn{2}{|c|}{$\begin{array}{c}\text { SC5314+FLC/ } \\
\text { SC5314 (A) }\end{array}$} & \multicolumn{2}{|c|}{$\begin{array}{c}u p c 2 \Delta / \Delta+F L C / \\
u p c 2 \Delta / \Delta(B)\end{array}$} & \multicolumn{2}{|c|}{ Ratio (B/A) } \\
\hline & & & $\operatorname{Exp1}$ & $\operatorname{Exp2}$ & Exp1 & $\operatorname{Exp2}$ & Exp1 & $\operatorname{Exp2}$ \\
\hline & orf19.4082 & DDR48 & 5.8 & 5.7 & 1.9 & 1.3 & 0.3 & 0.2 \\
\hline & orf19.4093 & PES1 & 2.7 & 3.5 & 0.6 & 1.3 & 0.2 & 0.4 \\
\hline & orf19.4317 & GRE3 & 1.5 & 1.5 & 0.3 & 0.7 & 0.2 & 0.5 \\
\hline & orf19.496 & & 2.6 & 2.3 & 1.1 & 0.6 & 0.4 & 0.3 \\
\hline & orf19.5902 & $R A S 2$ & 7.6 & 6.2 & 2.3 & 1.5 & 0.3 & 0.2 \\
\hline & orf19.7221 & SET3 & 4.6 & 2.9 & 2.1 & 1.4 & 0.5 & 0.5 \\
\hline & orf19.921 & $H M S 1$ & 2.6 & 2.8 & 0.8 & 1.3 & 0.3 & 0.5 \\
\hline \multirow{4}{*}{$\begin{array}{c}\text { Response to } \\
\text { chemical stimulus }\end{array}$} & orf19.4645 & BEMI & 1.5 & 1.9 & 0.4 & 0.6 & 0.3 & 0.3 \\
\hline & orf19.5591 & $A D O 1$ & 2.4 & 2.9 & 0.7 & 0.8 & 0.3 & 0.3 \\
\hline & orf19.6102 & $R C A 1$ & 2.5 & 3.4 & 1.1 & 1.4 & 0.5 & 0.4 \\
\hline & orf19.7374 & CTA4 & 1.7 & 2.2 & 0.8 & 1.0 & 0.5 & 0.5 \\
\hline \multirow{7}{*}{$\begin{array}{l}\text { Oxidation- } \\
\text { reduction process }\end{array}$} & orf19.1411 & & 2.7 & 4.8 & 1.4 & 1.1 & 0.5 & 0.2 \\
\hline & orf19.1710 & ALII & 2.0 & 1.9 & 0.7 & 0.8 & 0.4 & 0.4 \\
\hline & orf19.1940 & & 1.7 & 1.5 & 0.4 & 0.7 & 0.2 & 0.5 \\
\hline & orf19.2091 & & 2.2 & 2.3 & 0.8 & 0.9 & 0.4 & 0.4 \\
\hline & orf19.2108 & SOD6 & 7.9 & 10.6 & 1.1 & 1.6 & 0.1 & 0.1 \\
\hline & orf19.4274 & PUT1 & 6.8 & 8.8 & 2.5 & 0.6 & 0.4 & 0.1 \\
\hline & orf19.4747 & HEMI4 & 1.7 & 2.0 & 0.1 & 0.2 & 0.0 & 0.1 \\
\hline Filamentous & orf19.1300 & & 2.1 & 4.4 & 0.3 & 0.6 & 0.1 & 0.4 \\
\hline
\end{tabular}


Table 2-6. (Continued)

\begin{tabular}{|c|c|c|c|c|c|c|c|c|}
\hline \multirow[b]{3}{*}{ Process $^{a}$} & \multirow[b]{3}{*}{ orf19 Designation } & \multirow[b]{3}{*}{ CGD Name } & \multicolumn{4}{|c|}{ Change in Fold Expression } & & \\
\hline & & & \multicolumn{2}{|c|}{$\begin{array}{c}\text { SC5314+FLC/ } \\
\text { SC5314 (A) } \\
\end{array}$} & \multicolumn{2}{|c|}{$\begin{array}{c}u p c 2 \Delta / \Delta+\mathrm{FLC} / \\
\text { upc } 2 \Delta / \Delta \text { (B) }\end{array}$} & \multicolumn{2}{|c|}{ Ratio (B/A) } \\
\hline & & & Exp1 & $\operatorname{Exp2}$ & Exp1 & $\operatorname{Exp2}$ & Exp1 & $\operatorname{Exp2}$ \\
\hline \multirow[t]{3}{*}{ growth } & orf19.2809 & $C T N 3$ & 41.1 & 36.9 & 12.6 & 11.5 & 0.3 & 0.3 \\
\hline & orf19.3969 & SFL2 & 1.5 & 2.4 & 0.7 & 0.6 & 0.5 & 0.2 \\
\hline & orf19.5741 & $A L S 1$ & 66.8 & 79.0 & 4.8 & 3.1 & 0.1 & 0.0 \\
\hline \multirow{11}{*}{$\begin{array}{l}\text { Organelle } \\
\text { organization }\end{array}$} & orf19.109 & & 1.5 & 1.6 & 0.5 & 0.5 & 0.4 & 0.3 \\
\hline & orf19.2387 & & 1.7 & 1.5 & 0.7 & 0.7 & 0.4 & 0.4 \\
\hline & orf19.2984 & MST1 & 1.9 & 2.0 & 0.7 & 1.0 & 0.4 & 0.5 \\
\hline & orf19.3782.2 & & 1.5 & 1.8 & 0.6 & 0.5 & 0.4 & 0.3 \\
\hline & orf19.4051 & HTS1 & 1.8 & 1.7 & 0.9 & 0.8 & 0.5 & 0.5 \\
\hline & orf19.415 & & 2.2 & 2.3 & 1.2 & 1.0 & 0.5 & 0.5 \\
\hline & orf19.4273 & & 5.1 & 7.6 & 1.4 & 2.0 & 0.3 & 0.3 \\
\hline & orf19.4478 & & 1.6 & 1.8 & 0.5 & 0.8 & 0.3 & 0.5 \\
\hline & orf19.4657 & & 2.8 & 2.2 & 1.4 & 0.6 & 0.5 & 0.3 \\
\hline & orf19.5705 & NAM2 & 1.6 & 2.0 & 0.7 & 0.7 & 0.4 & 0.3 \\
\hline & orf19.6047 & $T U F 1$ & 1.8 & 2.8 & 0.3 & 0.3 & 0.2 & 0.1 \\
\hline \multirow{3}{*}{$\begin{array}{c}\text { DNA or RNA } \\
\text { metabolic process }\end{array}$} & orf19.1956 & & 1.5 & 1.8 & 0.8 & 1.0 & 0.5 & 0.5 \\
\hline & orf19.3138 & $N O P 1$ & 1.5 & 2.4 & 0.7 & 0.7 & 0.5 & 0.3 \\
\hline & orf19.7569 & SIK1 & 1.5 & 2.2 & 0.6 & 0.9 & 0.4 & 0.4 \\
\hline Translation & orf19.828 & & 1.8 & 1.5 & 0.2 & 0.4 & 0.1 & 0.3 \\
\hline
\end{tabular}


Table 2-6. (Continued)

\begin{tabular}{|c|c|c|c|c|c|c|c|c|}
\hline \multirow[b]{3}{*}{ Process $^{\mathrm{a}}$} & \multirow[b]{3}{*}{ orf19 Designation } & \multirow[b]{3}{*}{ CGD Name } & \multicolumn{4}{|c|}{ Change in Fold Expression } & & \\
\hline & & & \multicolumn{2}{|c|}{$\begin{array}{c}\text { SC5314+FLC/ } \\
\text { SC5314 (A) }\end{array}$} & \multicolumn{2}{|c|}{$\begin{array}{c}u p c 2 \Delta / \Delta+F L C / \\
u p c 2 \Delta / \Delta(\mathrm{B})\end{array}$} & \multicolumn{2}{|c|}{ Ratio (B/A) } \\
\hline & & & Exp1 & Exp2 & Exp1 & $\operatorname{Exp2}$ & Exp1 & Exp2 \\
\hline \multirow[t]{2}{*}{ Biological process } & orf19.4054 & CTA24 & 1.8 & 2.5 & 0.5 & 1.0 & 0.3 & 0.4 \\
\hline & orf19.6003 & & 2.4 & 2.1 & 0.9 & 0.7 & 0.4 & 0.4 \\
\hline \multirow{4}{*}{$\begin{array}{c}\text { Precursor } \\
\text { metabolites and } \\
\text { energy }\end{array}$} & orf19.1770 & CYCl & 1.9 & 2.2 & 0.6 & 0.7 & 0.3 & 0.3 \\
\hline & orf19.2439.1 & & 1.6 & 2.6 & 0.8 & 1.2 & 0.5 & 0.5 \\
\hline & orf19.3290 & & 1.8 & 2.0 & 1.0 & 1.1 & 0.5 & 0.5 \\
\hline & orf19.5893 & RIP1 & 1.6 & 2.2 & 0.7 & 0.8 & 0.5 & 0.4 \\
\hline \multirow{3}{*}{$\begin{array}{c}\text { Vitamin metabolic } \\
\text { process }\end{array}$} & orf19.2590 & & 1.8 & 2.8 & 0.0 & 0.2 & 0.0 & 0.1 \\
\hline & orf19.410.3 & & 5.6 & 5.7 & 1.0 & 0.8 & 0.2 & 0.1 \\
\hline & orf19.6057 & & 2.5 & 1.8 & 0.2 & 0.2 & 0.1 & 0.1 \\
\hline \multirow[t]{5}{*}{ Other } & orf19.2836 & & 3.4 & 5.9 & 1.6 & 1.5 & 0.5 & 0.3 \\
\hline & orf19.3008 & COQ4 & 2.5 & 1.8 & 0.4 & 0.8 & 0.2 & 0.5 \\
\hline & orf19.4899 & $G C A 1$ & 4.4 & 5.2 & 1.2 & 1.9 & 0.3 & 0.4 \\
\hline & orf19.6348 & & 3.0 & 2.4 & 0.6 & 1.2 & 0.2 & 0.5 \\
\hline & orf19.7263 & & 5.3 & 6.0 & 2.6 & 2.2 & 0.5 & 0.4 \\
\hline \multirow[t]{4}{*}{ Unknown } & orf19.1070 & & 8.6 & 8.0 & 2.4 & 1.6 & 0.3 & 0.2 \\
\hline & orf19.1277 & & 6.6 & 9.3 & 3.4 & 2.7 & 0.5 & 0.3 \\
\hline & orf19.1371 & & 1.5 & 1.6 & 0.8 & 0.6 & 0.5 & 0.4 \\
\hline & orf19.1440 & & 2.1 & 2.6 & 0.8 & 0.9 & 0.4 & 0.3 \\
\hline
\end{tabular}


Table 2-6. (Continued)

\begin{tabular}{|c|c|c|c|c|c|c|c|c|}
\hline \multirow[b]{3}{*}{ Process $^{a}$} & \multirow[b]{3}{*}{ orf19 Designation } & \multirow[b]{3}{*}{ CGD Name } & \multicolumn{4}{|c|}{ Change in Fold Expression } & & \\
\hline & & & \multicolumn{2}{|c|}{$\begin{array}{l}\text { SC5314+FLC/ } \\
\text { SC5314 (A) }\end{array}$} & \multicolumn{2}{|c|}{$\begin{array}{c}u p c 2 \Delta / \Delta+F L C / \\
u p c 2 \Delta / \Delta(B)\end{array}$} & \multicolumn{2}{|c|}{ Ratio (B/A) } \\
\hline & & & Exp1 & $\operatorname{Exp2}$ & $\operatorname{Exp1}$ & Exp2 & Exp1 & Exp2 \\
\hline & orf19.1562 & & 3.7 & 4.5 & 1.7 & 1.3 & 0.5 & 0.3 \\
\hline & orf19.2068 & & 1.6 & 1.5 & 0.6 & 0.6 & 0.4 & 0.4 \\
\hline & orf19.2414 & & 3.1 & 3.3 & 0.7 & 1.7 & 0.2 & 0.5 \\
\hline & orf19.2691 & & 1.5 & 1.5 & 0.4 & 0.7 & 0.3 & 0.5 \\
\hline & orf19.2839 & CIRT4B & 1.6 & 2.6 & 0.7 & 0.6 & 0.4 & 0.2 \\
\hline & orf19.287 & & 2.8 & 2.9 & 1.4 & 1.5 & 0.5 & 0.5 \\
\hline & orf19.3154 & & 2.1 & 2.3 & 0.6 & 0.6 & 0.3 & 0.2 \\
\hline & orf19.3499 & & 79.5 & 20.5 & 10.6 & 10.1 & 0.1 & 0.5 \\
\hline & orf19.3881 & & 1.9 & 2.0 & 0.5 & 0.5 & 0.2 & 0.2 \\
\hline & orf19.4013 & & 2.6 & 2.2 & 0.3 & 0.4 & 0.1 & 0.2 \\
\hline & orf19.4070 & & 5.0 & 8.9 & 1.8 & 1.9 & 0.4 & 0.2 \\
\hline & orf19.4144 & & 2.4 & 3.2 & 0.9 & 0.9 & 0.4 & 0.3 \\
\hline & orf19.4391 & & 3.2 & 1.5 & 1.0 & 0.7 & 0.3 & 0.5 \\
\hline & orf19.4658 & & 1.6 & 1.8 & 0.5 & 0.8 & 0.3 & 0.5 \\
\hline & orf19.467 & & 32.3 & 7.1 & 2.8 & 0.8 & 0.1 & 0.1 \\
\hline & orf19.5019 & & 3.5 & 3.7 & 0.9 & 1.9 & 0.2 & 0.5 \\
\hline & orf19.5205 & & 3.9 & 4.9 & 1.9 & 2.2 & 0.5 & 0.4 \\
\hline & orf19.5547 & & 2.8 & 2.4 & 0.8 & 1.1 & 0.3 & 0.4 \\
\hline & orf19.5635 & $P G A 7$ & 4.9 & 4.1 & 0.5 & 0.4 & 0.1 & 0.1 \\
\hline & orf19.6186 & & 12.1 & 13.3 & 2.8 & 2.7 & 0.2 & 0.2 \\
\hline & orf19.6187 & & 1.7 & 1.7 & 0.9 & 0.7 & 0.5 & 0.4 \\
\hline & orf19.6194 & & 1.5 & 1.6 & 0.6 & 0.9 & 0.4 & 0.5 \\
\hline
\end{tabular}


Table 2-6. (Continued)

\begin{tabular}{|c|c|c|c|c|c|c|c|c|}
\hline \multirow[b]{3}{*}{ Process $^{\mathrm{a}}$} & \multirow[b]{3}{*}{ orf19 Designation } & \multirow[b]{3}{*}{ CGD Name } & \multicolumn{4}{|c|}{ Change in Fold Expression } & & \\
\hline & & & \multicolumn{2}{|c|}{$\begin{array}{c}\text { SC5314+FLC/ } \\
\text { SC5314 (A) } \\
\end{array}$} & \multicolumn{2}{|c|}{$\begin{array}{c}u p c 2 \Delta / \Delta+F L C / \\
u p c 2 \Delta / \Delta(B)\end{array}$} & \multicolumn{2}{|c|}{ Ratio (B/A) } \\
\hline & & & Exp1 & Exp2 & Exp1 & Exp2 & Exp1 & Exp2 \\
\hline & orf19.6690 & & 2.4 & 2.3 & 0.6 & 0.7 & 0.3 & 0.3 \\
\hline & orf19.670.2 & & 47.4 & 152.7 & 18.3 & 14.1 & 0.4 & 0.1 \\
\hline & orf19.6713 & & 2.2 & 3.7 & 0.9 & 0.3 & 0.4 & 0.1 \\
\hline & orf19.6905 & & 2.4 & 3.3 & 1.2 & 1.4 & 0.5 & 0.4 \\
\hline & orf19.6970 & & 4.5 & 4.4 & 2.0 & 1.8 & 0.4 & 0.4 \\
\hline & orf19.6983 & & 2.1 & 9.1 & 0.4 & 0.0 & 0.2 & 0.0 \\
\hline & orf19.7380 & & 3.0 & 3.2 & 1.6 & 1.3 & 0.5 & 0.4 \\
\hline & orf19.7567 & & 1.9 & 2.1 & 0.2 & 0.2 & 0.1 & 0.1 \\
\hline & orf19.7675 & & 1.7 & 1.9 & 0.8 & 0.8 & 0.5 & 0.4 \\
\hline & orf19.951 & & 2.3 & 2.4 & 0.8 & 1.2 & 0.3 & 0.5 \\
\hline
\end{tabular}

${ }^{a}$ Descriptions are from the Candida Genome Database (http://www.candidagenome.org). 
for the microarray experiments. In addition to $E R G 11$, four others were chosen based on their involvement in iron transport and homeostasis. The correlation between the microarray data and that obtained by real time RT-PCR was good (Figure 2-5). The expressions of CFL4, FET3, FRP1, and FTR1 were upregulated in the wild type SC5314 when treated with fluconazole, but could not respond to the same extent when UPC2 was disrupted. As expected, ERG11 was also shown to respond to fluconazole in a UPC2dependent fashion.

These data suggest that the enhanced activity of fluconazole observed in both susceptible and resistant strains lacking UPC2 may be due to disregulation of iron homeostasis, in addition to the inability to upregulate genes involved in the ergosterol biosynthesis pathway.

\section{DISCUSSION}

Identifying novel drug targets that improve the efficacy of fluconazole is important in order to develop new therapeutic strategies to preserve the azole class of antifungals and overcome azole resistance. UPC2 has been well characterized with regard to its impact on fluconazole susceptibility and its role regulating genes of the ergosterol biosynthesis pathway (199-201, 224). Silver et al. and MacPherson et al. identified Upc2p as the key regulator of ergosterol metabolism in C. albicans, showing that azole-inducible expression of ERG2, ERG7, ERG11, and ERG25 is diminished in the absence of $U P C 2(199,200)$. We and others have established that in some azole-resistant isolates specific mutations render $U P C 2$ constitutively active, resulting in increased $E R G$ gene expression (including ERG11), cellular ergosterol levels, and increased fluconazole resistance $(156,168,201,203,204,216,219)$. This suggests that UPC2 influences azole susceptibility through the regulation of this pathway.

In the present study, we observed that not only did the disruption of UPC2 result in enhanced fluconazole susceptibility as measured by MIC, but also resulted in a substantial reduction in fluconazole MFC at 24, 48, and $72 \mathrm{hrs}$. Indeed disruption of $U P C 2$ in an azole-susceptible strain prevented its regrowth in YPD medium in the presence of higher fluconazole concentrations after 72 hours, resulted in a clear zone of inhibition around a fluconazole Etest strip, and prevented growth on solid media containing a therapeutically relevant concentration of fluconazole $(10 \mu \mathrm{g} / \mathrm{ml})$. Time-kill analysis also demonstrated an enhanced effect for fluconazole $10 \mu \mathrm{g} / \mathrm{ml}$ against the upc $2 \Delta / \Delta$ mutant as compared to its parent strain. Taken together, these data underscore the contribution of the Upc 2 transcriptional activation pathway to azole susceptibility.

We then wished to determine if disruption of UPC2 might have similar effects in fluconazole resistant isolates. For this purpose we chose isogenic strains containing resistance mutations in ERG11, MRR1, or TAC1. The strain containing the ERG1 $1^{\mathrm{K} 143 \mathrm{R}}$ mutation had an MIC of $8 \mu \mathrm{g} / \mathrm{ml}$ at 48 and 72 hours in YPD. Consistent with this, it was unable to grow in the presence of $10 \mu \mathrm{g} / \mathrm{ml}$ fluconazole. Although this background was not as highly resistant as others, its respective UPC2 deletion mutant had a marked drop 


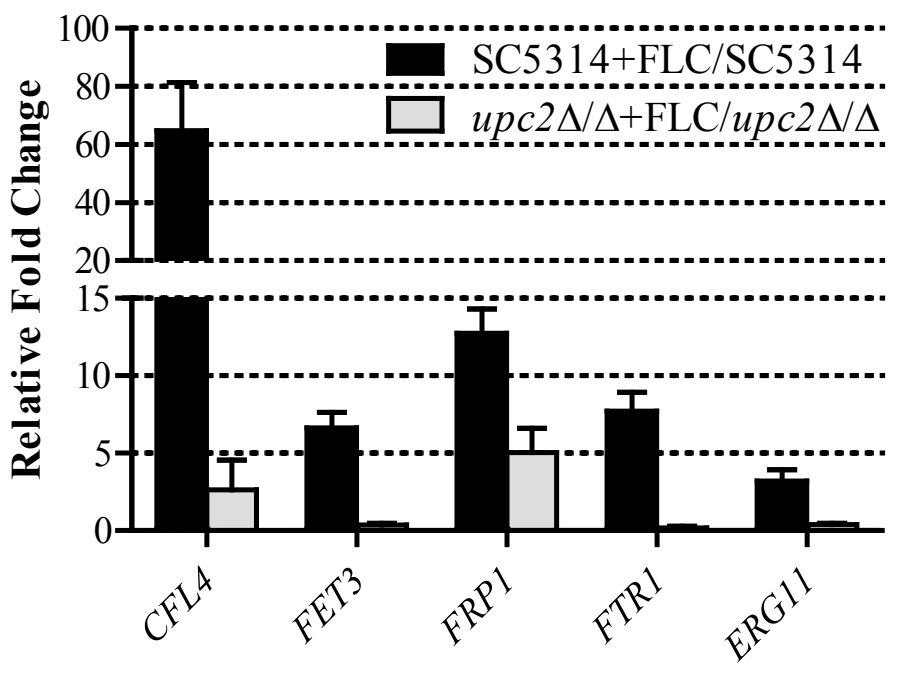

Figure 2-5. Validation of fluconazole-inducible and Upc2-dependent iron gene expression by $q R T-P C R$

Expression levels for CFL4, FET3, FRP1, FTR1, and ERG11 were measured in triplicate by qRT-PCR and compared to the expression levels in SC5314 in YPD. Shown are the relative $\mathrm{n}$-fold changes in gene expression in the in $\mathrm{SC} 5314$ and upc $2 \Delta / \Delta$ treated with fluconazole (FLC). Error bars represent the standard error of the mean. 
in both fluconazole MIC and MFC. The $M R R 1^{\mathrm{P} 683 \mathrm{~S}}$ and $T A C 1^{\mathrm{G} 980 \mathrm{E}}$ resistant strains both had an MIC and MFC of $>64 \mu \mathrm{g} / \mathrm{ml}$ at 48 and 72 hours in YPD medium which was reduced markedly in the respective upc $2 \Delta / \Delta$ mutants at all time points. This trend in susceptibility is consistent with what has been previously observed for this $M R R 1^{\mathrm{P} 683 \mathrm{~S}}$ upc $2 \Delta / \Delta$ mutant (214). However, unlike the halo of reduced confluent growth observed around the Etest strip with clinical isolate SC5314 (and generally observed with other $C$. albicans isolates), such growth was not observed with these resistant strains despite higher MICs and MFCs of $>64 \mu \mathrm{g} / \mathrm{ml}$. It has recently been shown that the $M R R 1^{\mathrm{P} 683 \mathrm{~S}}$ and $T A C 1^{\mathrm{G} 980 \mathrm{E}}$ constructed mutants exhibit a fitness defect associated with introduction of these specific resistance mutations, whereas clinical isolates carrying such mutations appear to have regained fitness (225). Such clinical isolates have likely evolved compensatory mutations which mitigate these fitness defects. This may explain the unusual growth pattern of these mutants when subjected to susceptibility testing by Etest. Despite the absence of reduced confluent growth, the upc $2 \Delta / \Delta$ mutants in each background showed increased susceptibility to fluconazole by Etest and an inability to grow in the presence of fluconazole at $10 \mu \mathrm{g} / \mathrm{ml}$. Therefore, the loss of $U P C 2$ in strains containing a single mechanism of resistance also resulted in enhanced susceptibility to fluconazole and to a greater extent than that observed for their susceptible parent strain SC5314.

The same phenotype was also observed upon disruption of UPC2 in a highly resistant clinical isolate, 12-99, which carries all four of the most common mechanisms of resistance. 12-99upc $2 \Delta / \Delta$ exhibited a substantial reduction in fluconazole resistance as compared to isolate 12-99. Although 12-99upc $2 \Delta / \Delta$ was able to resume growth in lower concentrations of fluconazole at 72 hours in broth microdilution plates, it was not able to grow to the extent of 12-99 or its susceptible matched isolate, 2-79, both of which were able to grow even in the highest concentration of fluconazole. Similarly to what was observed for $u p c 2 \Delta / \Delta$ in the SC5314 background, fluconazole exhibited a greater fungistatic effect against 12-99upc $2 \Delta / \Delta$ as compared to its parent azole-resistant isolate. Indeed the loss of UPC2 allowed fluconazole to overcome all four mechanisms of fluconazole resistance operative in isolate 12-99, and resulted in greater fluconazole susceptibility than was seen with its susceptible matched isolate, 2-79. Moreover, this resulted in a more pronounced effect by MFC, 72 hour regrowth, and time kill analysis than was observed for the susceptible wild type strain, SC5314.

One hypothesis for how the disruption of $U P C 2$ overcomes drug resistance is that it influences the expression of the efflux pump genes $C D R 1, C D R 2$, and $M D R 1$ in these strains. Indeed, it has been shown that in C. albicans Upc2 binds to the promoters of $C D R 1$ and MDRl and can regulate their expression (201). However, the expression of these genes was not affected in the absence of UPC2 in both the constructed mutant strains or resistant clinical isolate 12-99. The disruption of UPC2 had such an effect on susceptibility that even with constitutive upregulation of these efflux pumps, the strains were no longer resistant to fluconazole. $C D R 1$ and $C D R 2$ remained upregulated in $T A C 1^{\mathrm{G} 980 \mathrm{E}}$ and 12-99 as did MDRl in $M R R 1^{\mathrm{P} 683 \mathrm{~S}}$ and 12-99. 
Another possible explanation for the increased susceptibility to fluconazole observed in the upc $2 \Delta / \Delta$ mutants is a reduction in baseline or inducible expression of genes involved in sterol biosynthesis. In the presence of fluconazole, the ERG11 gene product, lanosterol demethylase, is inhibited leading to the accumulation of toxic sterol precursors and the production of alternate sterols such as lanosterol, eburicol, obtusifoliol, $14 \alpha$-methyl fecosterol and $14 \alpha$-methylergosta-8,24(28)-dien-3 $\beta, 6 \alpha$-diol (107). Integration of these sterols into the plasma membrane disrupts its integrity resulting in its altered structure and function $(79,82,226)$. Under these conditions, growth is inhibited but the organism remains viable (109). Fluconazole treatment also induces the expression of $E R G 11$ and other genes of the ergosterol biosynthesis pathway leading to increased production of not only lanosterol demethylase, but enzymes involved in alternate sterol production (227). However when UPC2 is disrupted, ERG11 expression would be reduced which would diminish the amount of drug target expressed in the cell. Expression of genes encoding enzymes needed for the biosynthesis of alternative sterols would also be reduced, further compromising the organism in the face of fluconazole exposure. Indeed expression of ERG11 was reduced in ERG1 lupc2 $\Delta / \Delta$, MRRIupc $2 \Delta / \Delta$ and TAClupc $2 \Delta / \Delta$ as well as $12-99 u p c 2 \Delta / \Delta$ as measured by real-time RT-PCR, and inducible expression of ERG6, ERG24, and ERG27 (as well as ERG11) was reduced in the upc $2 \Delta / \Delta$ mutant as compared to its parent strain SC5314 upon exposure to fluconazole as measured by microarray analysis. ERG6 and ERG27 both encode enzymes believed to be involved in the production of alternate sterols (Figure 2-6).

Of particular interest, another core set of fluconazole-induced genes we found to be dependent upon $U P C 2$ included those in the gene ontology process categories of iron transport (CFL4, FET3, FET34, FRE10, FRP1, FTR1) and iron homeostasis (CFL2, $C S A 1$, IRO1, RBT5). Iron starvation has been shown to enhance the susceptibility of $C$. albicans to fluconazole (228-231). Prasad et al. observed that an ftr $1 \Delta / \Delta$ mutant, which is defective in high-affinity iron uptake, also exhibited enhanced susceptibility to fluconazole (228). Upon examination of ERG11 mRNA abundance and membrane ergosterol levels of the $f \operatorname{tr} 1 \Delta / \Delta$ mutant, a reduction in $E R G 11$ expression and ergosterol content was observed compared to wild-type cells which led to increased membrane fluidity and consequently increased passive diffusion of fluconazole. In contrast $E R G 3$, which acts in the biosynthesis of $14 \alpha$-methylergosta-8,24(28)-dien-3 $\beta, 6 \alpha$-diol (232), was upregulated. This phenotype reverted upon the addition of iron to the medium. A similar observation has been made in Cryptococcus neoformans where disruption of the genes encoding the ferroxidase (CFOl) and an iron permease (CFTl) of the high-affinity reductive iron uptake system, resulted in increased susceptibility to azole antifungals (233).

As many enzymes in the ergosterol biosynthesis pathway require iron, it is tempting to speculate that decreased iron uptake, as suggested by the diminished expression of iron transport and homeostasis genes exhibited by the upc $2 \Delta / \Delta$ mutant relative to its parent strain in the presence of fluconazole, would have a significant impact on the activity of these enzymes. This, combined with decreased expression of the genes encoding enzymes involved in ergosterol biosynthesis, (ERG11 in particular), may 


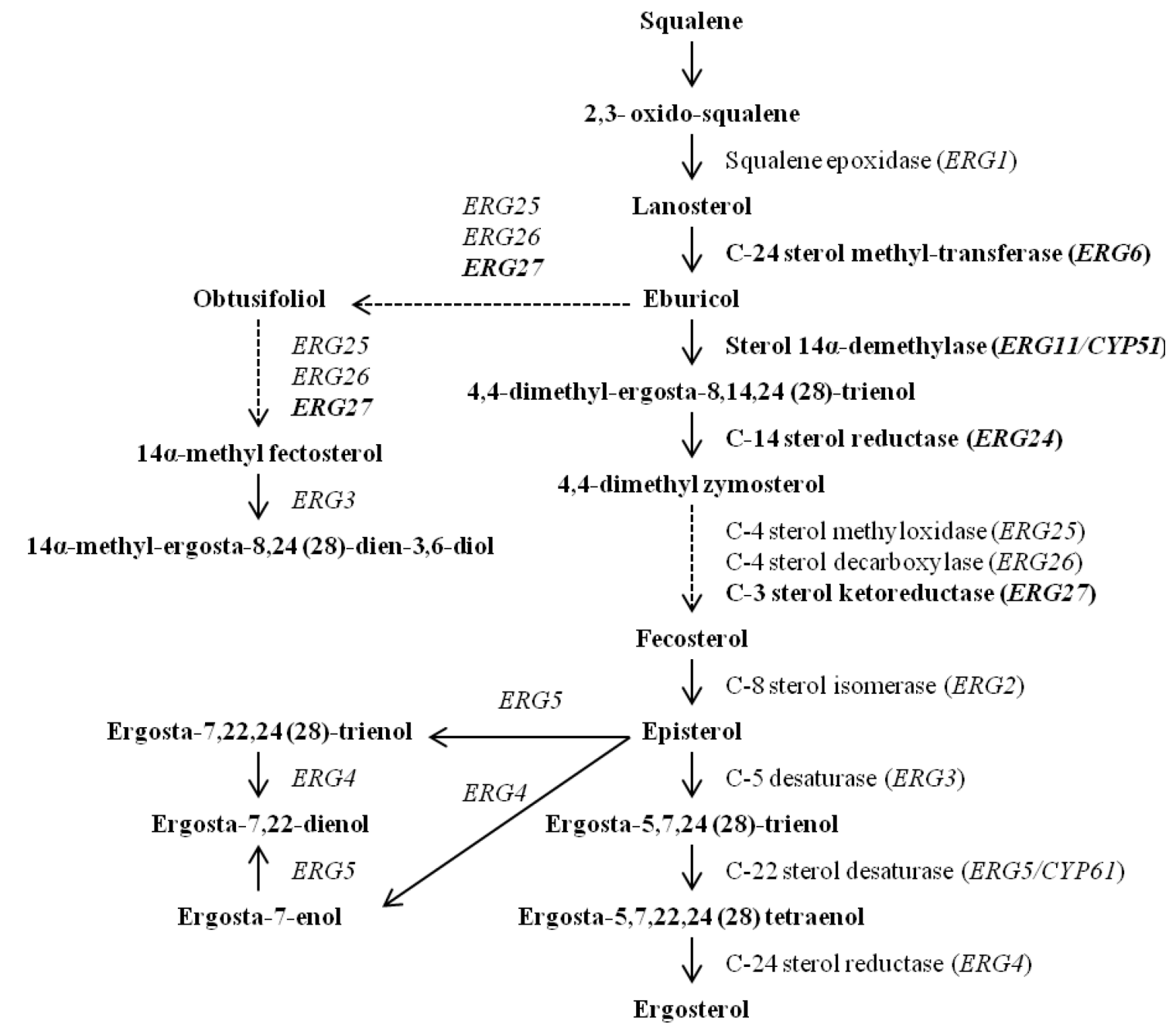

Figure 2-6. Sterol biosynthesis pathway in C. albicans

Genes in boldface were found to be responsive to fluconazole in a Upc2-dependent manner. Dotted lines denote multiple enzymatic steps $(232,234,235)$. 
account for the substantial effect on fluconazole susceptibility observed in these strains upon disruption of $U P C 2$. While further investigation is needed to determine which Upc2 targets influence susceptibility to the azole antifungals, our findings suggest that the Upc2 and the transcriptional activation pathway it regulates represents a potential target for overcoming azole antifungal resistance in C. albicans. 


\section{CHAPTER 3. CAS5 IMPACTS THE ACTIVITY OF FLUCONAZOLE AGAINST CANDIDA ALBICANS}

\section{INTRODUCTION}

Candida albicans is the most prevalent opportunistic human fungal pathogen, causing mucosal, cutaneous and systemic infections including oropharyngeal candidiasis (OPC), which is the most common opportunistic infection among AIDS patients (65). Candida species collectively are also the fourth-leading cause of nosocomial infections and are associated with high mortality rates $(208,209)$. The azole antifungals, particularly fluconazole (FLC), are the most widely used for treatment of Candida infections (65). However, C. albicans exhibits inhibited growth in the presence of azole antifungals, thus these agents are fungistatic against this organism (206). Identifying strategies to impart increased susceptibility to the azoles could improve their efficacy against $C$. albicans and may also diminish the development of resistance.

In C. albicans inhibition of the ergosterol biosynthesis pathway is not lethal suggesting that specific stress responses may circumvent or counter the activity of the azoles. Such an adaptive response would permit fungal survival in the presence of these antifungal agents. Indeed, in response to antifungal stress, specific signal transduction and transcriptional activation programs are affected (97). Drug efflux pump and ergosterol biosynthesis gene expression (236), the Ras-cAMP-PKA pathway (98), the calcineurin pathway (99-101), the Ssk1p/Chk1p pathway (102), and the cell wall integrity pathway $(105,237)$ have all been implicated as stress responses upon azole exposure yet the transcriptional activation pathways involved in these processes have yet to be identified. Moreover, it is likely that additional biological processes influence susceptibility to the azole antifungals.

Recently a library of C. albicans TF mutants was created and examined in 55 different growth conditions. Phenotypic profiling in response to various stress agents, including fluconazole, identified the possible biological roles of many previously uncharacterized TFs (187). While initial screens identified several TFs that affect fluconazole susceptibility, we undertook a more comprehensive analysis of this library in order to identify transcriptional activation programs that influence fluconazole activity and would therefore be implicated in the mechanisms by which C. albicans survives in the presence of the azoles. We identified one TF in particular, Cas5, whose disruption results enhanced azole activity indicating a role for the Cas5 transcriptional network in the susceptibility of C. albicans to the azole antifungal agents. 


\section{MATERIALS AND METHODS}

\section{Strains and Growth Conditions}

All C. albicans strains (Table 3-1) were stored as frozen stock in $40 \%$ glycerol at $-80^{\circ} \mathrm{C}$. YPD ( $1 \%$ yeast extract, $2 \%$ peptone, and $1 \%$ dextrose) agar plates and YPD liquid medium were used for routine growth of strains at $30^{\circ} \mathrm{C}$. For colony forming unit $(\mathrm{CFU})$ counts during time-kill analysis, PDA $(0.4 \%$ potato starch, $2 \%$ dextrose, and $1.5 \%$ agar) plates were used, and cultures grown on PDA were incubated at $35^{\circ} \mathrm{C}$.

\section{Drug Susceptibility Testing}

The minimum inhibitory concentrations (MICs) of fluconazole were determined by using broth microdilution as described by the Clinical and Laboratory Standards Institute M27-A2, modified by using both RPMI and YPD media, and were read both visually and spectrophotometrically at 24, 48, and 72 hours. Minimum fungicidal concentrations (MFCs) were measured by removing $2 \mu \mathrm{l}$ from each well of the MIC plate and plating onto SD or YPD agar, respectively. Also, serial dilutions from a $0.1 \mathrm{OD}_{600}$ suspension were diluted 4-fold and $2 \mu \mathrm{l}$ of each dilution plated onto YPD agar plates with and without $10 \mu \mathrm{g} / \mathrm{ml}$ fluconazole and then incubated at $30^{\circ} \mathrm{C}$ for 24 and 48 hours. Fluconazole activity was also assessed by Epsilometer test strips (Etest strips) (Biomerieux) according to manufacturer's instructions with the following modifications. A standardized cell suspension (0.5 McFarland) was used to create a confluent lawn across YPD agar plates prior to Etest strip placement and then incubated at $30^{\circ} \mathrm{C}$ for 24

and 48 hours. Time-kill analyses were performed with a $0.5 \mathrm{McF}$ arland cell suspension, which was 10-fold diluted into YPD media with and without $10 \mu \mathrm{g} / \mathrm{ml}$ fluconazole and incubated at $35^{\circ} \mathrm{C}$. Aliquots were removed at $0,6,12$, and 24 hours, 10 -fold serial diluted and plated onto PDA. CFUs were counted in duplicate after 48 hours at $35^{\circ} \mathrm{C}$ and plotted on a log-scale curve versus time (238).

\section{Construction of Gene Deletion Strains}

Plasmid pBSS2 contains the entire $S A T 1$-flipper disruption cassette from pSFS2 in a pBluescript vector backbone. The SAT1-flipper cassette consists of the SAT1 selectable marker which confers resistance to nourseothricin and the FLP flipper recombinase gene both flanked by $F R T$ sites (flipper recombinase target sequences). The target gene's 5' flanking sequence was cloned upstream of the SAT1-flipper cassette while the 3' flanking sequence was cloned downstream of the $S A T 1$-flipper cassette (Table 3-2). Upon transformation into the SC5314 strain the SAT1-flipper was inserted into the coding region of one allele, and such positive transformants $\left(\mathrm{Nou}^{\mathrm{R}}\right)$ were selected on YPD-nourseothricin agar plates containing $200 \mu \mathrm{g} / \mathrm{ml}$ of nourseothricin. Induction of the FLP gene occurred by growing the transformants in YPD medium for 24 hours without selective pressure. Positive cells $\left(\mathrm{Nou}^{\mathrm{S}}\right)$ were selected by replica plating onto 
Table 3-1. C.albicans strains used in this study

\begin{tabular}{|c|c|c|c|c|}
\hline Strain & Designation & $\begin{array}{c}\text { Strain } \\
\text { Background }\end{array}$ & $\begin{array}{c}\text { Relevant Characteristics or } \\
\text { Genotype }\end{array}$ & Source \\
\hline SC5314 & SC5314 & $\mathrm{N} / \mathrm{A}$ & $\begin{array}{c}C A S 5 / C A S 5 \text { CZF1/CZF1 } \\
M K C 1 / M K C 1 \text { RPN4/RPN4 }\end{array}$ & ATCC \\
\hline SCCAS5A5A66C & $\operatorname{cas} 5 \Delta / \Delta$ & SC5314 & cas $5 \Delta:: \mathrm{FRT} /$ cas $5 \Delta:: \mathrm{FRT}$ & This study \\
\hline SCCAS5A5A66C1B & $\operatorname{cas} 5 \Delta / \Delta+C A S 5$ & SCCAS5A5A66C & cas $5 \Delta:: \mathrm{FRT} / C A S 5$-caSAT1 & This study \\
\hline SCRPN4A1A21C & $\operatorname{rpn} 4 \Delta / \Delta$ & SC5314 & 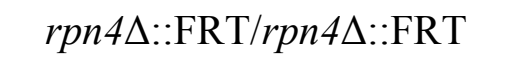 & This study \\
\hline SCRPN4A1A21C6A & $r p n 4 \Delta / \Delta+R P N 4$ & SCRPN4A1A21C & $r p n 4 \Delta:: \mathrm{FRT} / R P N 4-\mathrm{ca} S A T 1$ & This study \\
\hline SCCZF1G1D12 & $c z f 1 \Delta / \Delta$ & SC5314 & $c z f 1 \Delta::$ FRT/czfl $1 \Delta::$ FRT & This study \\
\hline SCMKC1B5A4A & $m k c 1 \Delta / \Delta$ & SC5314 & $m k c 1 \Delta:: \mathrm{FRT} / m k c 1 \Delta:: \mathrm{FRT}$ & This study \\
\hline SCMKC1B5A4A9A & $m k c 1 \Delta / \Delta+M K C 1$ & SCMKC1B5A4A & $m k c 1 \Delta:: \mathrm{FRT} / M K C 1$-caSAT1 & This study \\
\hline Clinical isolate2-79 & $2-79$ & $\mathrm{~N} / \mathrm{A}$ & Susceptible isolate & Redding, 1994 \\
\hline Clinical isolate12-99 & $12-99$ & $\mathrm{~N} / \mathrm{A}$ & Resistant isolate & Redding, 1994 \\
\hline 12-99CAS5A1A72 & $12-99 \operatorname{cas} 5 \Delta / \Delta$ & $12-99$ & cas $5 \Delta:: \mathrm{FRT} / \operatorname{cas} 5 \Delta:: \mathrm{FRT}$ & This study \\
\hline $\mathrm{G} 2$ & $\mathrm{G} 2$ & G1 & Susceptible isolate & Franz, 1998 \\
\hline G5 & G5 & $\mathrm{G} 2$ & Resistant isolate & Franz, 1998 \\
\hline G5CAS5B16D3D & $\mathrm{G} 5 \operatorname{cas} 5 \Delta / \Delta$ & G5 & 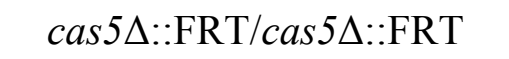 & This study \\
\hline
\end{tabular}


Table 3-1. (Continued)

\begin{tabular}{|c|c|c|c|c|}
\hline Strain & Designation & $\begin{array}{c}\text { Strain } \\
\text { Background }\end{array}$ & $\begin{array}{l}\text { Relevant Characteristics or } \\
\text { Genotype }\end{array}$ & Source \\
\hline $\mathrm{Gu} 2$ & Gu2 & Gul & Susceptible isolate & Franz, 1999 \\
\hline Gu5 & Gu5 & Gu2 & Resistant isolate & Franz, 1999 \\
\hline Gu5CAS5A23A4A & $\mathrm{Gu} 5 \operatorname{cas} 5 \Delta / \Delta$ & Gu5 & $\operatorname{cas} 5 \Delta:: \mathrm{FRT} / \operatorname{cas} 5 \Delta:: \mathrm{FRT}$ & This study \\
\hline $\mathrm{S} 1$ & S1 & $\mathrm{N} / \mathrm{A}$ & Susceptible isolate & Franz, 1999 \\
\hline S2 & S2 & S1 & Resistant isolate & Franz, 1999 \\
\hline S2CAS5B6C5A & $\mathrm{S} 2 \operatorname{cas} 5 \Delta / \Delta$ & S2 & $\operatorname{cas} 5 \Delta:: \mathrm{FRT} / \operatorname{cas} 5 \Delta:: \mathrm{FRT}$ & This study \\
\hline SCUPC2R14A & $U P C 2^{\mathrm{G} 648 \mathrm{D}}$ & SC5314 & $\begin{array}{c}U P C 2^{\mathrm{G} 648 \mathrm{D}}:: \mathrm{FRT} / U P C 2^{\mathrm{G} 648 \mathrm{D}}:: \mathrm{FRT} \\
\\
C A S 5 / C A S 5\end{array}$ & Heilmann, 2010 \\
\hline SCUPC2R14ACAS5A5A21Y & $U P C 2 \operatorname{cas} 5 \Delta / \Delta$ & SCUPC2R14A & $\begin{array}{c}U P C 2^{\mathrm{G} 648 \mathrm{D}}:: \mathrm{FRT} / U P C 2^{\mathrm{G} 648 \mathrm{D}}:: \mathrm{FRT} \\
\text { cas } 5 \Delta:: \mathrm{FRT} / \text { cas } 5 \Delta:: \mathrm{FRT}\end{array}$ & This study \\
\hline 10C1B1M1 & $E R G 11^{\mathrm{K} 143 \mathrm{R}}$ & SC5314 & ERG1 $1^{K 143 R}::$ FRT/ERG1 ${ }^{K 143 R}::$ FRT & Chapter 2 \\
\hline 10C1B1M1CAS5-1A51B & ERG11cas $5 \Delta / \Delta$ & 10C1B1M1 & 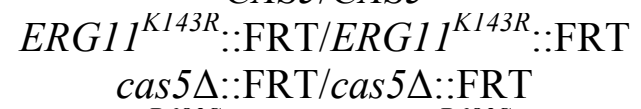 & This study \\
\hline SCMRR1R34A & $M R R 1^{\mathrm{P} 683 \mathrm{~S}}$ & SC5314 & $\begin{array}{c}M R R 1^{P 683 S}:: \text { FRT/MRRI } I^{P 683 S}:: \mathrm{FRT} \\
\quad C A S 5 / C A S 5\end{array}$ & Schubert, 2011 \\
\hline SCMRR1R34ACAS5-2A3 & MRRIcas5 $5 / \Delta$ & SCMRR1R34A & $\begin{array}{l}\text { MRR } 1^{P 683 S}:: \text { FRT/MRR } 1^{P 683 S}:: \text { FRT } \\
\text { cas5 } 5:: \text { FRT/cas } 5 \Delta:: \text { FRT }\end{array}$ & This study \\
\hline SCTAC1R34A & $T A C 1^{\mathrm{G} 980 \mathrm{E}}$ & SC5314 & $T^{T A C 1^{G 9800}}::$ FRT $/ T A C 1^{G 9800}::$ FRT & Sasse, 2011 \\
\hline SCTAC1R34ACAS5-2A1 & TAClcas $5 \Delta / \Delta$ & SCTAC1R34A & 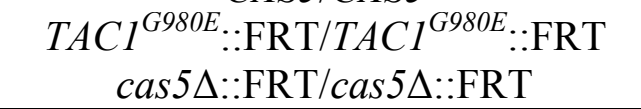 & This study \\
\hline
\end{tabular}


Table 3-2. Primers used in this study

\begin{tabular}{|c|c|c|}
\hline Method & Primer & Sequence \\
\hline \multirow{20}{*}{ qRT-PCR } & $A C T 1-\mathrm{F}$ & 5'-ACGGTGAAGAAGTTGCTGCTTTAGTT-3' \\
\hline & $A C T 1-\mathrm{R}$ & 5'-CGTCGTCACCGGCAAAA-3' \\
\hline & $B M R I-\mathrm{F}$ & 5'-ACATAAATACTTTGCCCATCCAGAA-3' \\
\hline & $B M R I-\mathrm{R}$ & 5'-AAGAGTTGGTTTGTAATCGGCTAAA-3' \\
\hline & CDRI-F & 5'-ATTCTAAGATGTCGTCGCAAGATG-3' \\
\hline & $C D R 1-\mathrm{R}$ & 5'-AGTTCTGGCTAAATTCGTAATGTTTTC-3' \\
\hline & $C D R 2-\mathrm{F}$ & 5'-TAGTCCATTCAACGGCAACATT-3' \\
\hline & CDR2-R & 5'-CACCCAGTATTTGGCATTGAAA-3' \\
\hline & CFL4-F & 5'-GCAATGGTTGACAGGTTGGAA-3' \\
\hline & CFL4-R & 5'-GCAATGTGACGATGATAAGTGACAA-3' \\
\hline & $E R G 11-\mathrm{F}$ & 5'-ССССТАTTAATTTTGTTTTCCCTAATTTAC-3' \\
\hline & $E R G 11-\mathrm{R}$ & 5'-CACGTTCTCTTCTCAGTTTAATTTCTTTC-3' \\
\hline & $E R G 26-\mathrm{F}$ & 5'-ATTAGAGTGTGCGATGCCATT-3' \\
\hline & $E R G 26-\mathrm{R}$ & 5'-CGTCGTCGTAGTCGTCAC-3' \\
\hline & FTR1-F & 5'-ATTGTTGTTTCAGTGCTTTTGGC-3' \\
\hline & FTRl-R & 5'-GGTCGGAACTACCACCCATAGA-3' \\
\hline & $P G A 13-\mathrm{F}$ & 5'-CTCCGAAGAAGAAGGAAGCGT-3' \\
\hline & $P G A 13-\mathrm{R}$ & 5'-GGTGAAATCAGTGACGGTGACTT-3' \\
\hline & $P G A 31-\mathrm{F}$ & 5'-CTTTCCTTTGATGGTTCCGACT-3' \\
\hline & $P G A 31-\mathrm{R}$ & 5'-GGGTCATTGATGTTCTTGGCA-3' \\
\hline \multirow{19}{*}{$\begin{array}{c}\text { Mutant } \\
\text { construction }\end{array}$} & CAS5-A & 5'-TTCAACTTCAAGGTACCTGTTGATATTCTA-3' \\
\hline & CAS5-B & 5'-CTAACGGTCTCGAGAAATTTTGTGATAGTT-3' \\
\hline & CAS5-C & 5'-ATTGTGGTAACCGCGGTAATCGTAAAGATA-3' \\
\hline & CAS5-D & 5'-GCATATTTAGGAGCTCCTAGTGGTGATTTA-3' \\
\hline & CAS5-E & 5'-GCATACTGCATATTTAGCTCGAGCTAGTGG-3' \\
\hline & RPN4-A & 5'-AGATCAATATAGGGCCCTAAAGGACATCTT-3' \\
\hline & $R P N 4-\mathrm{B}$ & 5'-GTGTATTATTGGCTCGAGTAAAAGTGTTGT-3' \\
\hline & RPN4-C & 5'-TAGATTTAATCCGCGGAGCTAAAGAGAATG-3' \\
\hline & $R P N 4-\mathrm{D}$ & 5'-CTGGTGAAGAGCTCTAAAGTGATATTATTG-3' \\
\hline & RPN4-E & 5'-CTGGTGAAGAACTCGAGAGTGATATTATTG-3' \\
\hline & CZF1-A & 5'-GCAAAATTCAAAGGGCCCGACCTAATTC-3' \\
\hline & $C Z F 1-\mathrm{B}$ & 5'-GTGGTTGTGCTCGAGGAGAGGTATTGG-3' \\
\hline & $C Z F 1-\mathrm{C}$ & 5'-CAATTGAAGCGGCCGCGTTTGGTAGAATC-3' \\
\hline & $C Z F 1-\mathrm{D}$ & 5'-GTCAACTTTTGCCGCGGCACACACGTAAA-3' \\
\hline & MKC1-A & 5'-TTTGATAATAAAAAGGGGCCCTTGAGAA-3' \\
\hline & $M K C 1-\mathrm{B}$ & 5'-GATAAATTCCTGGCTCGAGACCTTATTTAC-3' \\
\hline & $M K C 1-\mathrm{C}$ & 5'-AAGAGTATATAAAGCCCGCGGAAGAGCTTG-3' \\
\hline & $M K C 1-\mathrm{D}$ & 5'-AACAAGACTGAGCTCAACAAAAGAAATGTA-3' \\
\hline & $M K C 1-\mathrm{E}$ & 5'-AACAAGACTGAACTCGAGAAAAGAAATGTA-3' \\
\hline
\end{tabular}

Underlined sequence reflects the introduction of a restriction site sequence. 
YPD plates with and without $200 \mu \mathrm{g} / \mathrm{ml}$ of nourseothricin. Upon induction of the FLP gene, the cassette was excised such that only one copy of the $F R T$ site remains in the locus. Another round was required to disrupt the second allele (34).

For gene complementation, the target gene's open reading frame (ORF) $\pm \sim 1 \mathrm{~kb}$ was cloned in place of the target gene's 5' flanking sequence in the aforementioned disruption cassette. Upon transformation into the SC5314 strain, one allele of the gene's coding region was inserted into the original locus in conjunction with the $S A T 1$-flipper, and such positive transformants $\left(\mathrm{Nou}^{\mathrm{R}}\right)$ were selected on YPD-nourseothricin agar plates containing $200 \mu \mathrm{g} / \mathrm{ml}$ of nourseothricin. Induction of the $F L P$ gene occurred by growing the transformants in YPD medium for 24 hours without selective pressure. Positive cells $\left(\mathrm{Nou}^{\mathrm{S}}\right)$ were selected on by replica plated onto YPD plates with and without $200 \mu \mathrm{g} / \mathrm{ml}$ of nourseothricin. Upon induction of the FLP gene, the cassette is excised such that only the gene's allele with a downstream $F R T$ was left in the locus. Appropriate gene disruption and complementation was confirmed by Southern hybridization (34).

\section{Isolation of Genomic DNA and Southern Hybridization}

Genomic DNA was isolated as described previously (217). Four $\mu \mathrm{g}$ of DNA were digested with an appropriate restriction endonuclease, separated on a 1\% agarose gel, and after staining with ethidium bromide, were transferred by vacuum blotting onto a nylon membrane and fixed by UV cross-linking. Southern hybridization with enhanced chemiluminescence-labeled probes was performed with the Amersham ECL Direct nucleic acid labeling and detection system according to the instructions of the manufacturer.

\section{RNA Isolation for qRT-PCR}

RNA was isolated using a small-scale hot phenol method of RNA isolation described by Schmitt et al (218). Briefly, overnight cultures were diluted to an $\mathrm{OD}_{600}$ of 0.2 in $20 \mathrm{ml} \mathrm{YPD}$ and then incubated at $30^{\circ} \mathrm{C}$ with shaking for 3 hours. Cells were collected by centrifugation, and stored at $-80^{\circ} \mathrm{C}$. Cell pellets were resuspended in $950 \mu \mathrm{l}$ of AE buffer and then transferred to a 2-ml RNase-free microcentrifuge tube containing $950 \mu 1$ acid phenol (pH 4.3) with $1 \%$ SDS. Cells were incubated at $65^{\circ} \mathrm{C}$ for 10 minutes then lysates were clarified by centrifugation. The supernatant was then divided into two new 2-ml microcentrifuge tubes containing $950 \mu 1$ of chloroform and mixed. The sample was then subjected to centrifugation again, and the top aqueous layer was transferred to a new tube containing $1 \mathrm{ml}$ of isopropanol and $100 \mu \mathrm{l} 2 \mathrm{M}$ sodium acetate. The RNA pellet was subsequently washed with $500 \mu 1$ of $70 \%$ ethanol and collected by centrifugation. The RNA pellet was resuspended in DNase/RNase-free $\mathrm{H}_{2} \mathrm{O}$. Quantity and purity were determined spectrophotometrically at absorbances of $\mathrm{A}_{260}$ and $\mathrm{A}_{280}$. 


\section{Quantitative RT-PCR}

First-strand cDNAs were synthesized from $1 \mu \mathrm{g}$ of total RNA using SuperScript first-strand synthesis system for qRT-PCR (Invitrogen). Gene-specific primers (Table 3-2) were designed using Primer Express software (Applied Biosystems) and synthesized by Integrated DNA Technologies (Coralville, IA). Quantitative PCRs were performed in triplicate using the 7000 sequence detection system (Applied Biosystems), independently amplifying ACT1 (normalizing gene) and the genes of interest (GOI) as described previously (219).

\section{RNA Isolation for Microarray}

RNA was isolated using a large-scale version of the hot phenol method of RNA isolation described by Schmitt et al. (218). Briefly, overnight cultures were diluted to an $\mathrm{OD}_{600}$ of 0.005 in $100 \mathrm{ml} \mathrm{YPD}$ and then incubated at $30^{\circ} \mathrm{C}$ with shaking for an additional 8 hours to an $\mathrm{OD}_{600}$ of 1.0. Cultures were diluted again to an $\mathrm{OD}_{600}$ of 0.025 in $100 \mathrm{ml}$ fresh YPD, allowed to incubate at $30^{\circ} \mathrm{C}$ with shaking for one doubling, inoculated with or without $10 \mu \mathrm{g} / \mathrm{ml}$ fluconazole, and then incubated at $30^{\circ} \mathrm{C}$ with shaking for 6 hours. Cells were collected by centrifugation, and stored at $-80^{\circ} \mathrm{C}$. Cell pellets were resuspended in $12 \mathrm{ml}$ of $\mathrm{AE}$ buffer and then transferred to a 50-ml Oak Ridge tubes treated with RNAse Away (Molecular BioProducts) containing $12 \mathrm{ml}$ acid phenol (pH 4.3 ) with $1 \%$ SDS. Cells were incubated at $65^{\circ} \mathrm{C}$ for 10 minutes then lysates were clarified by centrifugation. The supernatant was then transferred to a new tube containing $15 \mathrm{ml}$ of chloroform and mixed. The sample was then subjected to centrifugation again, and the top aqueous layer was transferred to a new tube containing one volume of isopropanol and 0.1 volume $2 \mathrm{M}$ sodium acetate. The RNA pellet was subsequently washed with $10 \mathrm{ml}$ of $70 \%$ ethanol and collected by centrifugation. The RNA pellet was resuspended in DNase/RNase-free $\mathrm{H}_{2} \mathrm{O}$. Quantity and purity were determined spectrophotometrically at absorbances of $\mathrm{A}_{260}$ and $\mathrm{A}_{280}$.

\section{Transcriptional Profiling}

Gene expression profiles were obtained by hybridizing labeled cRNAs generated from $C$. albicans total RNA onto Affymetrix C. albicans custom expression arrays (CAN07; 49-5241) (216), which have been described previously (201). Microarray hybridization and analysis were performed as described previously (201). Genes were considered to be differentially expressed in response to drug if their expression changed by $\geq 1.5$-fold in two independent experiments. Genes induced by fluconazole were considered to be $C A S 5$-dependent if the induction was abrogated in the deletion mutant was $\geq 2.0$-fold (50\%) less than the wild-type. All microarray data is available for download from the NCBI at GEO (Accession number: pending). 


\section{RESULTS}

\section{Disruption of Cas5 Enhances Fluconazole Activity against $\boldsymbol{C}$. albicans}

In order to identify transcriptional activation programs that influence azole susceptibility of $C$. albicans to the azole antifungals, we first screened a collection of TF deletion mutants for those that displayed a increase in fluconazole susceptibility as measured by broth microdilution in RPMI medium (187). In order to detect marked reductions in MIC, a lower range of fluconazole concentrations from 0.0071825 to 4 $\mu \mathrm{g} / \mathrm{ml}$ were used, and $19 \mathrm{TFs}$ were identified from the library with reductions in fluconazole MIC in RPMI medium. These hits were then rescreened for those that also displayed a reduction in fluconazole MFC in both RPMI and YPD media (Table B-1). We identified four TF mutants that met the criteria: CAS5, RPN4, UPC2 and CZF1 (Table 3-3). The observation that disruption of UPC2 increased susceptibility to fluconazole was not surprising as this has been reported previously $(199,200)$, validating the results of our screen. The remaining three TF deletion mutants which exhibited a reduction in MFC in both RPMI and YPD were prioritized as being of greatest interest and independent mutants were generated in the SC5314 background (Table 3-4). In order to determine the extent to which disruption of these TF genes impacts fluconazole susceptibility, the deletion mutants were subjected to various susceptibility tests using the nutrient rich YPD media in order to detect strong phenotypes despite existing in an environment that promotes growth.

CAS5 and RPN4 disruption had a moderate impact on fluconazole MIC, and a marked impact on MFC by broth microdilution (Table 3-4) and Etest (Figure 3-1A), while $C Z F 1$ had no impact by all methods. The fluconazole MFC at 24 hours in YPD for $\mathrm{SC} 5314$ was $>64 \mu \mathrm{g} / \mathrm{ml}$ whereas the $\operatorname{cas} 5 \Delta / \Delta, r p n 4 \Delta / \Delta$, and $c z f 1 \Delta / \Delta$ mutants were 1,16 , and $>64 \mu \mathrm{g} / \mathrm{ml}$ respectively (Table 3-4), indicating that $C Z F 1$ could not be independently confirmed as influencing susceptibility to fluconazole and therefore did not proceed through any further examination. For SC5314, the 48 hour MIC by Etest was $1.0 \mu \mathrm{g} / \mathrm{ml}$ and a halo of reduced growth was observed up to the Etest strip consistent with the fungistatic nature of fluconazole. At 48 hours, the MICs by Etest for the cas $5 \Delta / \Delta$ and $r p n 4 \Delta / \Delta$ mutant were $0.25 \mu \mathrm{g} / \mathrm{ml}$ and $1.5 \mu \mathrm{g} / \mathrm{ml}$ respectively. A large clear zone of inhibition was observed for cas $5 \Delta / \Delta$ but was smaller for $r p n 4 \Delta / \Delta$ (Figure 3-1A). Using a 72 hour endpoint for a broth microdilution assay in YPD as a way to assess the ability of the organism to resume growth in the presence of fluconazole we found that SC5314 and $r p n 4 \Delta / \Delta$ were able to resume growth in all concentrations of fluconazole tested whereas the cas $5 \Delta / \Delta$ mutant only grew at the lowest concentrations (Figure 3-1B). When plated on YPD agar plates containing $10 \mu \mathrm{g} / \mathrm{ml}$ of fluconazole, growth was reduced for the cas $5 \Delta / \Delta$ mutant, but only slightly for $r p n 4 \Delta / \Delta$, as compared to SC5314 (Figure 3-1C). Time-kill analysis showed increased fungistatic activity for $10 \mu \mathrm{g} / \mathrm{ml}$ fluconazole against the cas $5 \Delta / \Delta$ mutant, but not $r p n 4 \Delta / \Delta$, as compared to their parent strain (Figure 3-1D). RPN4 was not pursued in further analysis due to its minimal impact at the therapeutically relevant concentration of fluconazole, $10 \mu \mathrm{g} / \mathrm{ml}$. All phenotypes were reverted by reintegration of one allele of the disrupted gene. 
Table 3-3. Library hits with reduced MICs and MFCs in both RPMI and YPD

\begin{tabular}{|c|c|c|c|c|c|c|}
\hline \multirow[b]{2}{*}{ Clone ID } & \multirow[b]{2}{*}{ CGD } & \multirow[b]{2}{*}{ orf19 Designation } & \multicolumn{2}{|c|}{$\mathrm{MIC}(\mu \mathrm{g} / \mathrm{ml})$} & \multicolumn{2}{|c|}{ MFC $(\mu \mathrm{g} / \mathrm{ml})$} \\
\hline & & & RPMI & YPD & RPMI & YPD \\
\hline $\mathrm{WT}$ & -- & -- & 0.25 & 0.5 & $>4$ & $>64$ \\
\hline TF3 & RPN4 & orf19.1069 & 0.0625 & 0.25 & 0.125 & 32 \\
\hline TF33 & $C A S 5$ & orf19.4670 & 0.125 & 0.25 & 4 & 2 \\
\hline TF77 & $U P C 2$ & orf19.391 & 0.03125 & 0.125 & 1 & 1 \\
\hline TF104 & $C Z F 1$ & orf19.3127 & 0.0625 & 0.5 & 0.25 & 8 \\
\hline
\end{tabular}


Table 3-4. MICs and MFCs in YPD in SC5314 background

\begin{tabular}{|c|c|c|c|c|c|c|c|}
\hline \multirow[b]{2}{*}{ Strain } & \multirow{2}{*}{$\begin{array}{c}\text { Relevant Characteristics } \\
\text { or Genotype }\end{array}$} & \multicolumn{3}{|c|}{$\mathrm{MIC}(\mu \mathrm{g} / \mathrm{ml})$} & \multicolumn{3}{|c|}{ MFC $(\mu \mathrm{g} / \mathrm{ml})$} \\
\hline & & $24 \mathrm{hr}$ & $48 \mathrm{hr}$ & $72 \mathrm{hr}$ & $24 \mathrm{hr}$ & $48 \mathrm{hr}$ & $72 \mathrm{hr}$ \\
\hline SC5314 & CAS5/CAS5 CZF1/CZF1 & 0.5 & 0.5 & $>64$ & $>64$ & $>64$ & $>64$ \\
\hline $\operatorname{cas} 5 \Delta / \Delta$ & $\begin{array}{c}M K C 1 / M K C 1 \text { RPN4/RPN4 } \\
\text { cas5 } 5:: \mathrm{FRT} / \text { cas } 5 \Delta:: \mathrm{FRT}\end{array}$ & 0.5 & 1 & 1 & 1 & 1 & 1 \\
\hline $\operatorname{cas} 5 \Delta / \Delta+C A S 5$ & cas5 $\Delta:: \mathrm{FRT} / C A S 5$-caSAT1 & 0.5 & 0.5 & $>64$ & $>64$ & $>64$ & $>64$ \\
\hline $\operatorname{rpn} 4 \Delta / \Delta$ & 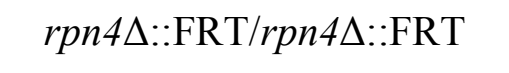 & 0.5 & 1 & $>64$ & 8 & 8 & 8 \\
\hline$r p n 4 \Delta / \Delta+R P N 4$ & $r p n 4 \Delta:: \mathrm{FRT} / R P N 4$-caSAT1 & 0.5 & 0.5 & $>64$ & $>64$ & $>64$ & $>64$ \\
\hline$c z f 1 \Delta / \Delta$ & $c z f 1 \Delta:: \mathrm{FRT} / c z f 1 \Delta:: \mathrm{FRT}$ & 0.5 & 0.5 & $>64$ & $>64$ & $>64$ & $>64$ \\
\hline$m k c 1 \Delta / \Delta$ & $m k c 1 \Delta::$ FRT $/ m k c 1 \Delta::$ FRT & 0.5 & 0.5 & 1 & 1 & 1 & 1 \\
\hline$m k c 1 \Delta / \Delta+M K C 1$ & $m k c 1 \Delta:: \mathrm{FRT} / M K C 1-\mathrm{ca} S A T 1$ & 0.5 & 0.5 & $>64$ & $>64$ & $>64$ & $>64$ \\
\hline
\end{tabular}


Figure 3-1. Effects of CAS5 and RPN4 on fluconazole susceptibility in SC5314 background

(A) Effect on MIC and growth on YPD agar as determined by Etest. A confluent lawn of C. albicans was streaked prior to the addition of Etest strips and then incubated for 48 hours. (B) MIC heat map of SC5314, mutants, and complimented derivative. Susceptibility was determined by broth microdilution in YPD at 72 hours. Growth was quantified spectrophotometrically and assigned to a colorimetric scale. (C) Effect on the ability to grow on solid media containing fluconazole. From 4-fold serial dilutions of $C$. albicans strains, $2 \mu \mathrm{l}$ aliquots were spotted onto YPD agar $+/-10 \mu \mathrm{g} / \mathrm{mL}$ FLC and incubated for 48 hours. (D) Effect of fluconazole by time-kill assay. SC5314, cas $5 \Delta / \Delta$, or $r p n 4 \Delta / \Delta$ cells were diluted in YPD medium containing FLC $(10 \mu \mathrm{g} / \mathrm{mL})$ or the solvent, DMSO. After 0, 6, 12, and 24 hours, samples from each were diluted and plated for colony forming units. 


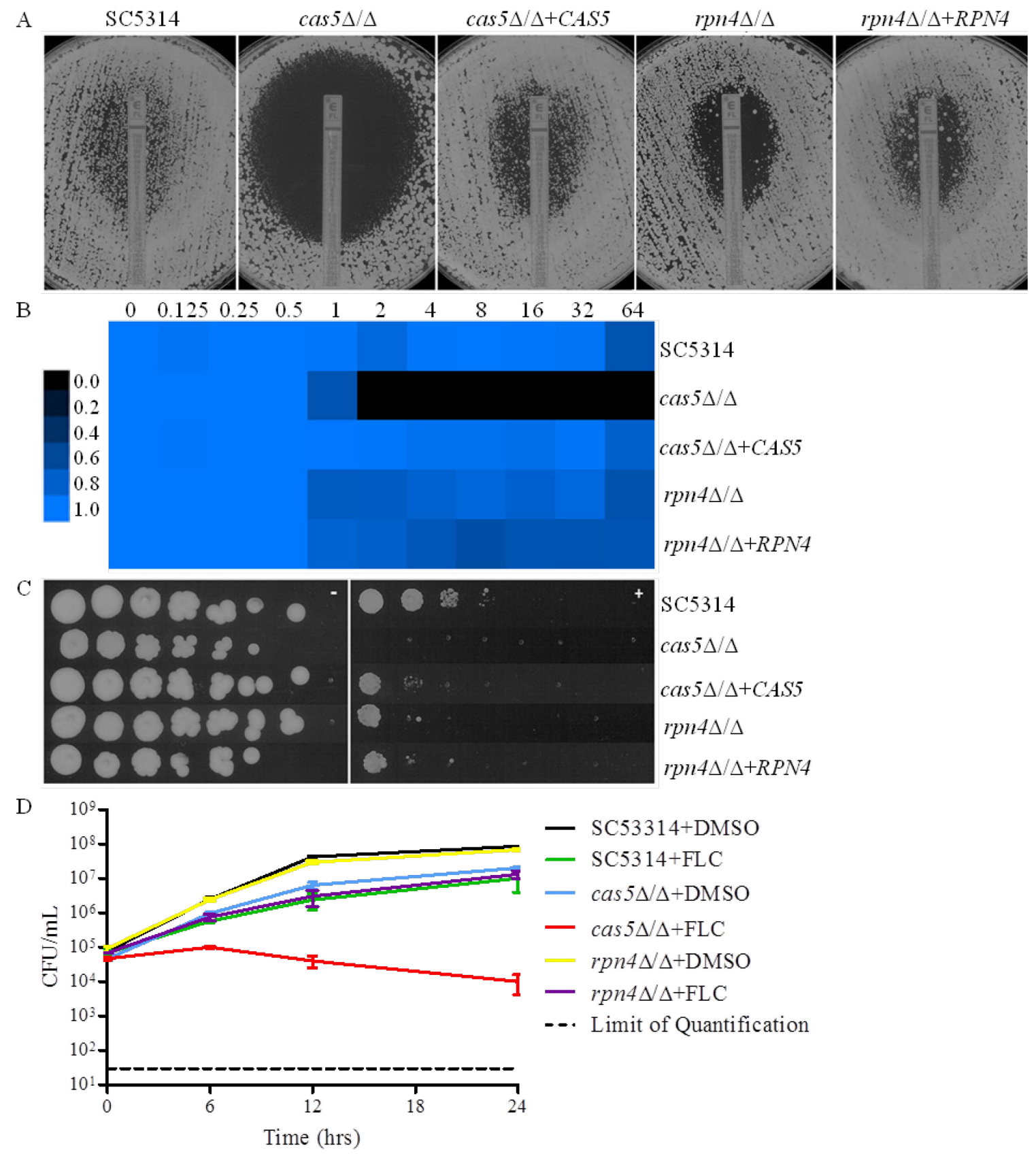




\section{MKC1 Disruption Does Not Fully Phenocopy CAS5}

The cell wall integrity (CWI) pathway has been previously shown to be critical for the activity of sterol biosynthesis inhibitors and is specific to fungal cells (103-105). This pathway is conserved in C. albicans from Saccharomyces cerevisiae and in $C$. albicans the transcription factor Cas $5 \mathrm{p}$ is postulated to be the functional equivalent of the $S$. cerevisiae CWI transcriptional regulator $\operatorname{Rlm} 1$ (134, 239). Looking upstream of Rlm1 in the CWI pathway is Slt2, a stress-activated kinase phosphorylated by Pkc1p. The functional equivalent of this protein in C. albicans is $\mathrm{Mkcl}$ and is required for the maintenance of cell wall integrity as well as the azole stress response in C. albicans (103, 240, 241). In order to determine if deletion of $M K C 1$ in SC5314 phenocopies deletion of $C A S 5$, we similarly tested an $m k c 1 \Delta / \Delta$ mutant for susceptibility to fluconazole.

Disruption of $M K C 1$ had no change in fluconazole MIC at 24 or 48 hours, but a marked impact on MFC by broth microdilution (Table 3-4), and MIC by Etest (Figure 3-2A). The fluconazole MFC at 24 hours in YPD for SC5314 was $>64 \mu \mathrm{g} / \mathrm{ml}$ whereas the $m k c l \Delta / \Delta$ mutant was $1 \mu \mathrm{g} / \mathrm{ml}$ (Table 3-4). At 48 hours, the MIC by Etest for SC5314 was $0.5 \mu \mathrm{g} / \mathrm{ml}$ and a halo of reduced growth was observed up to the Etest strip consistent with the fungistatic nature of fluconazole. Meanwhile, the $m k c 1 \Delta / \Delta$ mutant had an MIC by Etest of $0.25 \mu \mathrm{g} / \mathrm{ml}$ and a large clear zone of inhibition was observed (Figure 3-2A). We also used a 72 hour endpoint for a broth microdilution assay as a way to assess the ability of the organism to regrow in the presence of fluconazole. SC5314 and $m k c 1 \Delta / \Delta+M K C 1$ were able to resume growth in all concentrations of fluconazole tested whereas the $m k c 1 \Delta / \Delta$ mutant only grew at the lowest concentrations (Figure 3-2B). When plated on YPD agar plates containing $10 \mu \mathrm{g} / \mathrm{ml}$ fluconazole, growth was reduced in the presence of fluconazole for the $m k c 1 \Delta / \Delta$ mutant as compared to SC5314 (Figure 3-2C). Time-kill analysis showed slightly increased activity of $10 \mu \mathrm{g} / \mathrm{ml}$ fluconazole against the $m k c 1 \Delta / \Delta$ mutant as compared to the parent strain, but some regrowth was observed after 24 hours (Figure 3-2D). All phenotypes were reverted by reintegration of one allele of the disrupted gene.

\section{Disruption of Cas5 Does Not Override Combinations of Clinical Drug Resistance Mechanisms}

In order to examine the extent at which Cas $5 \mathrm{p}$ is involved, we constructed $\operatorname{cas} 5 \Delta / \Delta$ mutant strains in the background of an azole-resistant clinical isolate (12-99) known to carry four of the most common mechanisms of azole resistance: overexpression of $C D R 1$ and $C D R 2$, overexpression of $M D R 1$, overexpression of $E R G 11$, and a mutation in ERG11 (242). The disruption of CAS5 in isolate 12-99 did not result in a reduction in $\mathrm{MIC}$ or MFC, and therefore was not able to overcome the combined mechanisms of resistance operative in this isolate (Table 3-5). At 48 hours, the MIC by Etest for 12-99 was $>256 \mu \mathrm{g} / \mathrm{ml}$ and confluent growth was observed up to the Etest strip consistent with the resistance of 12-99 to fluconazole, and the same was observed for $12-99 \operatorname{cas} 5 \Delta / \Delta$, although this strain exhibited slower growth (data not shown). Time-kill analysis 
Figure 3-2. $\quad M K C 1$ effects fluconazole susceptibility in SC5314 background (A) Effect of $\mathrm{MKCl}$ on MIC and growth on YPD agar as determined by Etest. A confluent lawn of $C$. albicans was streaked prior to the addition of Etest strips and then incubated for 48 hours. (B) MIC heat map of SC5314, mutant, and complimented derivative. Susceptibility was determined by broth microdilution in YPD at 72 hours. Growth was quantified spectrophotometrically and assigned to a colorimetric scale. (C) Effect of $\mathrm{MKCl}$ on the ability to grow on solid media containing fluconazole. From 4fold serial dilutions of $C$. albicans strains, $2 \mu \mathrm{l}$ aliquots were spotted onto YPD agar +/$10 \mu \mathrm{g} / \mathrm{mL}$ FLC and incubated for 48 hours. (D) Effect of fluconazole on $\mathrm{MKCl}$ in timekill assay. SC5314 or $m k c 1 \Delta / \Delta$ cells were diluted in YPD medium containing FLC (10 $\mu \mathrm{g} / \mathrm{mL}$ ) or the solvent, DMSO. After $0,6,12$, and 24 hours, samples from each were diluted and plated for colony forming units. 


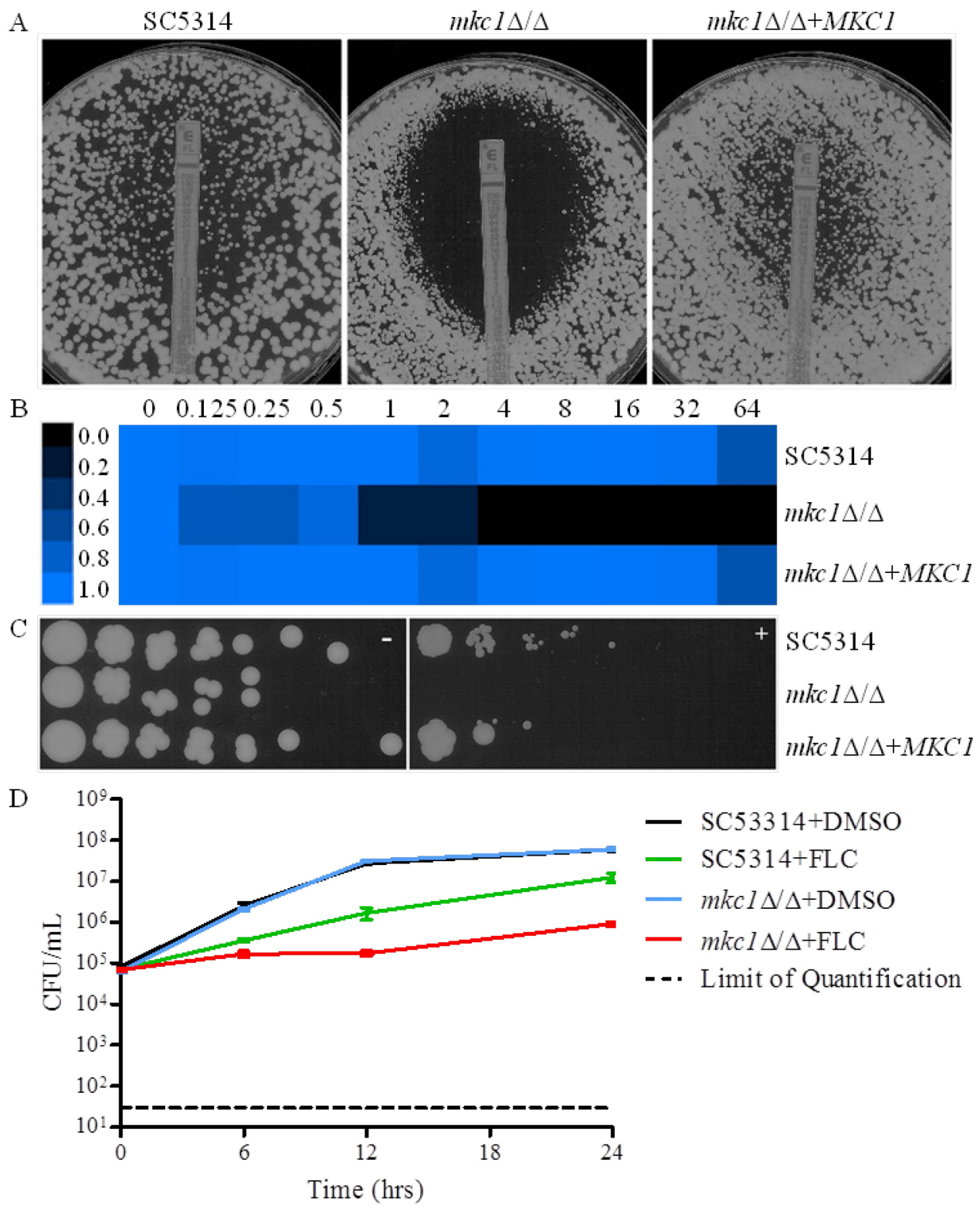


Table 3-5. MICs and MFCs in YPD in background of clinical resistant strains

\begin{tabular}{|c|c|c|c|c|c|c|c|}
\hline \multirow[b]{2}{*}{ Strain } & \multirow[b]{2}{*}{$\begin{array}{c}\text { Relevant Characteristics } \\
\text { or Genotype }\end{array}$} & \multicolumn{3}{|c|}{ MIC ( $(\mu \mathrm{g} / \mathrm{ml})$} & \multicolumn{3}{|c|}{$\operatorname{MFC~}(\mu \mathrm{g} / \mathrm{ml})$} \\
\hline & & $24 \mathrm{hr}$ & $48 \mathrm{hr}$ & $72 \mathrm{hr}$ & $24 \mathrm{hr}$ & $48 \mathrm{hr}$ & $72 \mathrm{hr}$ \\
\hline SC5314 & $C A S 5 / C A S 5$ & 0.5 & 0.5 & $>64$ & $>64$ & $>64$ & $>64$ \\
\hline $2-79$ & Susceptible isolate & 1 & 1 & $>64$ & $>64$ & $>64$ & $>64$ \\
\hline $12-99$ & Resistant isolate & $>64$ & $>64$ & $>64$ & $>64$ & $>64$ & $>64$ \\
\hline $12-99$ cas $5 \Delta / \Delta$ & cas5 $5::$ FRT/cas $5 \Delta::$ FRT & $>64$ & $>64$ & $>64$ & $>64$ & $>64$ & $>64$ \\
\hline $\mathrm{G} 2$ & Susceptible isolate & 1 & $>64$ & $>64$ & $>64$ & $>64$ & $>64$ \\
\hline G5 & Resistant isolate & $>64$ & $>64$ & $>64$ & $>64$ & $>64$ & $>64$ \\
\hline $\mathrm{G} 5 \operatorname{cas} 5 \Delta / \Delta$ & cas $5 \Delta::$ FRT/cas $5 \Delta::$ FRT & 32 & 32 & 32 & $>64$ & $>64$ & $>64$ \\
\hline Gu2 & Susceptible isolate & 0.5 & 1 & $>64$ & $>64$ & $>64$ & $>64$ \\
\hline Gu5 & Resistant isolate & $>64$ & $>64$ & $>64$ & $>64$ & $>64$ & $>64$ \\
\hline $\mathrm{Gu} 5 \operatorname{cas} 5 \Delta / \Delta$ & cas5 $5::$ FRT/cas $5 \Delta::$ FRT & $>64$ & $>64$ & $>64$ & $>64$ & $>64$ & $>64$ \\
\hline S1 & Susceptible isolate & 2 & $>64$ & $>64$ & $>64$ & $>64$ & $>64$ \\
\hline $\mathrm{S} 2$ & Resistant isolate & $>64$ & $>64$ & $>64$ & $>64$ & $>64$ & $>64$ \\
\hline $\mathrm{S} 2 \operatorname{cas} 5 \Delta / \Delta$ & 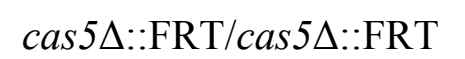 & 64 & $>64$ & $>64$ & $>64$ & $>64$ & $>64$ \\
\hline
\end{tabular}


revealed an overall growth defect but this did not alter the effect of fluconazole against $12-99 \operatorname{cas} 5 \Delta / \Delta$ at $10 \mu \mathrm{g} / \mathrm{ml}$ (Figure 3-3).

Isolate 12-99 simultaneously overexpresses $C D R 1$ and $C D R 2$ as well as $M D R 1$ (156). Since previous studies have shown that azole resistant isolates usually do not simultaneously overexpress these transporter genes $(154,167,243), C A S 5$ was also disrupted in the clinical isolates $\mathrm{S} 2, \mathrm{G} 5$, and Gu5. Isolate $\mathrm{S} 2$ has been previously shown to have a G464S amino acid substitution in Upc2p which renders it constitutively active, resulting in upregulation of ERG11 and increased fluconazole resistance $(195,202)$. Isolate G5 has a G997V amino acid substitution in Mrr1p, resulting in increased MDR1 expression and is also homozygous for an ERG11 mutation $(152,195)$. Isolate Gu5 displays increased expression of $C D R 1$ and $C D R 2$ (153). As with isolate 12-99, the disruption of CAS5 in these three clinical isolates did not markedly alter the MIC or MFC in these resistant backgrounds when examined by broth microdilution (Table 3-5). The only change observed was a 24 hour MIC of $32 \mu \mathrm{g} / \mathrm{ml}$ for G5cas $5 \Delta / \Delta$ reduced from $>64$ $\mu \mathrm{g} / \mathrm{ml}$ by broth microdilution, and a 48 hour Etest MIC of $24 \mu \mathrm{g} / \mathrm{ml}$ reduced from $>256$ $\mu \mathrm{g} / \mathrm{ml}$ (data not shown).

\section{Disruption of $C A S 5$ in Strains Containing Resistance Mutations in MRR1, TAC1, and $E R G 11$ Impacts Fluconazole Susceptibility}

Since the deletion of CAS5 was unable to override the common resistance mechanisms acting together we sought to investigate the requirement for $C A S 5$ for specific mechanisms of resistance. Independent mutants were constructed in background strains containing two copies of a gene conferring reduced susceptibility to fluconazole: $M R R 1^{\mathrm{P} 683 \mathrm{~S}}, T A C 1^{\mathrm{G} 980 \mathrm{E}}, U P C 2^{\mathrm{G} 648 \mathrm{D}}$ or $E R G 11^{\mathrm{K} 143 \mathrm{R}}$. The $M R R 1^{\mathrm{P} 683 \mathrm{~S}}, T A C 1^{\mathrm{G} 980 \mathrm{E}}$ and $U P C 2^{\mathrm{G} 648 \mathrm{D}}$ alleles contain gain-of-function mutations which render the transcription factors they encode constitutively active, resulting in upregulation of $M D R 1, C D R 1$ and $C D R 2$, and $E R G 11$ respectively, and decreased fluconazole susceptibility $(195,196,202$, 220, 221). The ERG $11^{\mathrm{K} 143 \mathrm{R}}$ allele contains a point mutation postulated to be located near the azole access channel, interfering with entry of fluconazole, resulting in decreased fluconazole susceptibility (222). Interestingly, we observed the UPC2cas $5 \Delta / \Delta$ mutant cells to be larger than those of the parent strain and also to be elongated and clumped together. As a result, susceptibility testing for this strain was inconsistent due to the extreme variability in optical density versus cell density, and therefore could not be reported. The disruption of $C A S 5$ in each remaining background did not markedly impact the MICs at 24 hours, but did result in a reduction in MIC at later time points by all methods. The MFCs at 48 hours in YPD for ERG11cas $52 \Delta / \Delta, M R R 1$ cas $5 \Delta / \Delta$ and TAC1cas $5 \Delta / \Delta$ were all reduced from $8 \mu \mathrm{g} / \mathrm{ml},>64 \mu \mathrm{g} / \mathrm{ml}$, and $>64 \mu \mathrm{g} / \mathrm{ml}$ in their background strains to $4 \mu \mathrm{g} / \mathrm{ml}, 8 \mu \mathrm{g} / \mathrm{ml}$, and $16 \mu \mathrm{g} / \mathrm{ml}$ respectively (Table 3-6). At 48 hours the MICs by Etest for ERG11 cas $5 \Delta / \Delta$, MRR1cas $5 \Delta / \Delta$ and TAC1cas $5 \Delta / \Delta$ were all reduced from $1.5 \mu \mathrm{g} / \mathrm{ml}, 6 \mu \mathrm{g} / \mathrm{ml}$, and $8 \mu \mathrm{g} / \mathrm{ml}$ in their background strains to $0.5 \mu \mathrm{g} / \mathrm{ml}$, $1.5 \mu \mathrm{g} / \mathrm{ml}$, and $6 \mu \mathrm{g} / \mathrm{ml}$ respectively (Figure 3-4A). When plated on YPD agar plates containing $10 \mu \mathrm{g} / \mathrm{ml}$ fluconazole, growth was slightly reduced in the presence of 


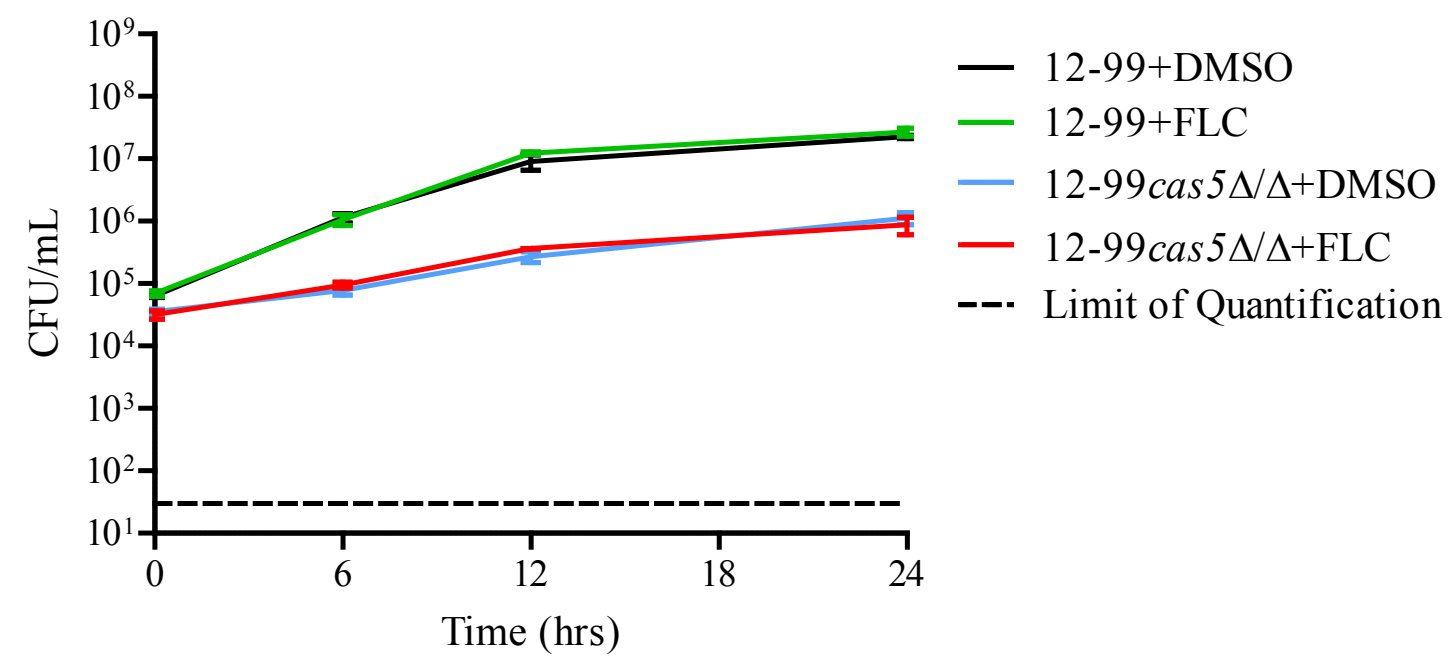

Figure 3-3. Effect of fluconazole on CAS5 in 12-99 by time-kill assay

$12-99$ or $12-99$ cas $5 \Delta / \Delta$ cells were diluted in YPD medium containing FLC $(10 \mu \mathrm{g} / \mathrm{mL})$ or the solvent, DMSO. After 0, 6, 12, and 24 hours, samples from each were diluted and plated for colony forming units. 
Table 3-6. MICs and MFCs in YPD in background of strains expressing resistance mechanisms

\begin{tabular}{|c|c|c|c|c|c|c|c|}
\hline \multirow[b]{2}{*}{ Strain } & \multirow[b]{2}{*}{$\begin{array}{c}\text { Relevant Characteristics or } \\
\text { Genotype }\end{array}$} & \multicolumn{3}{|c|}{ MIC $(\mu \mathrm{g} / \mathrm{ml})$} & \multicolumn{3}{|c|}{ MFC $(\mu \mathrm{g} / \mathrm{ml})$} \\
\hline & & $24 \mathrm{hr}$ & $48 \mathrm{hr}$ & $72 \mathrm{hr}$ & $24 \mathrm{hr}$ & $48 \mathrm{hr}$ & $72 \mathrm{hr}$ \\
\hline SC5314 & $C A S 5 / C A S 5$ & 0.5 & 0.5 & $>64$ & $>64$ & $>64$ & $>64$ \\
\hline$E R G 11^{\mathrm{K} 143 \mathrm{R}}$ & $\begin{array}{c}E R G 11^{K 143 R}:: \mathrm{FRT} / E R G 11^{K 143 R}:: \mathrm{FRT} \\
\text { CAS5/CAS5 }\end{array}$ & 4 & 8 & 8 & 4 & 8 & 8 \\
\hline $\begin{array}{c}E R G 11 \operatorname{cas} 5 \Delta / \\
\Delta\end{array}$ & $\begin{array}{c}\text { ERG } 11^{K 143 R}:: \mathrm{FRT} / E R G 11^{K 143 R}:: \mathrm{FRT} \\
\operatorname{cas} 5 \Delta:: \mathrm{FRT} / \operatorname{cas} 5 \Delta:: \mathrm{FRT}\end{array}$ & 4 & 4 & 4 & 4 & 4 & 4 \\
\hline$M R R 1^{\mathrm{P} 683 \mathrm{~S}}$ & $\begin{array}{c}M R R 1^{P 683 S}:: \text { FRT/MRR1 } \\
\text { CAS5 } 5 / C A S 5\end{array}$ & 8 & 16 & $>64$ & $>64$ & $>64$ & $>64$ \\
\hline MRRlcas $5 \Delta / \Delta$ & $\begin{array}{c}M R R 1^{P 683 S}:: \mathrm{FRT} / M R R 1^{P 683 S}:: \mathrm{FRT} \\
\operatorname{cas}^{2} 5:: \mathrm{FRT} / \text { cas }^{2} \Delta:: \mathrm{FRT}\end{array}$ & 8 & 8 & 8 & 8 & 8 & 8 \\
\hline$T A C 1^{\mathrm{G} 980 \mathrm{E}}$ & $\begin{array}{c}T A C 1^{G 980 E}:: \mathrm{FRT} / T A C 1^{G 980 E}:: \mathrm{FRT} \\
C A S 5 / C A S 5\end{array}$ & 8 & 16 & $>64$ & $>64$ & $>64$ & $>64$ \\
\hline$T A C 1 \operatorname{cas} 5 \Delta / \Delta$ & $\begin{array}{c}\text { TAC1 } 1^{G 980 E}:: \mathrm{FRT} / T A C 1^{G 980 E}:: \mathrm{FRT} \\
\text { cas } 5 \Delta:: \mathrm{FRT} / \text { cas } 5 \Delta:: \mathrm{FRT}\end{array}$ & 8 & 16 & 16 & 16 & 16 & 16 \\
\hline
\end{tabular}




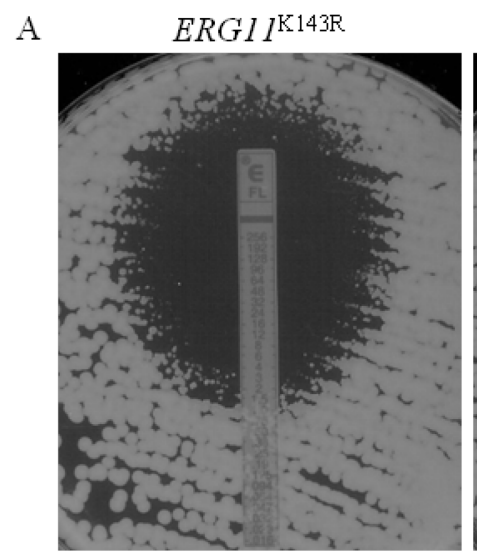

ERG1 lcas $5 \Delta / \Delta$

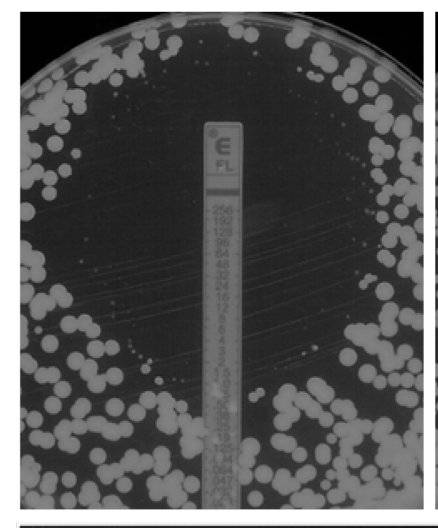

$\mathrm{B}$

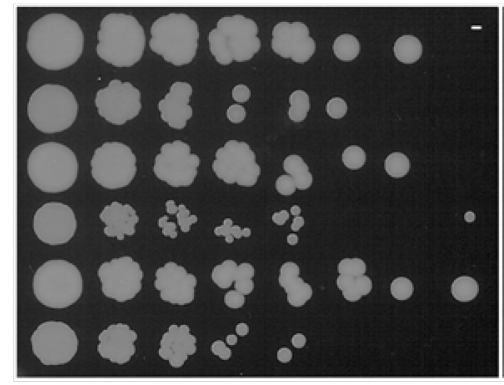

$M R R l^{\mathrm{P} 683 \mathrm{~S}}$

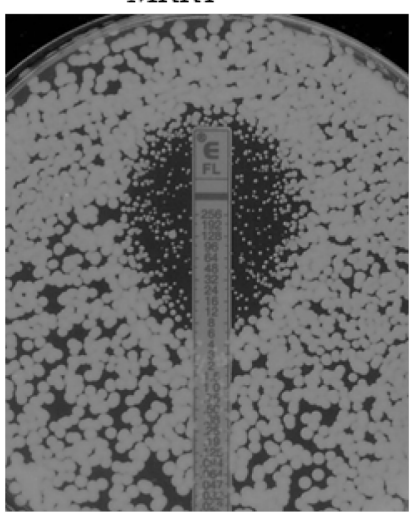

$\operatorname{MRRICas} 5 \Delta / \Delta$
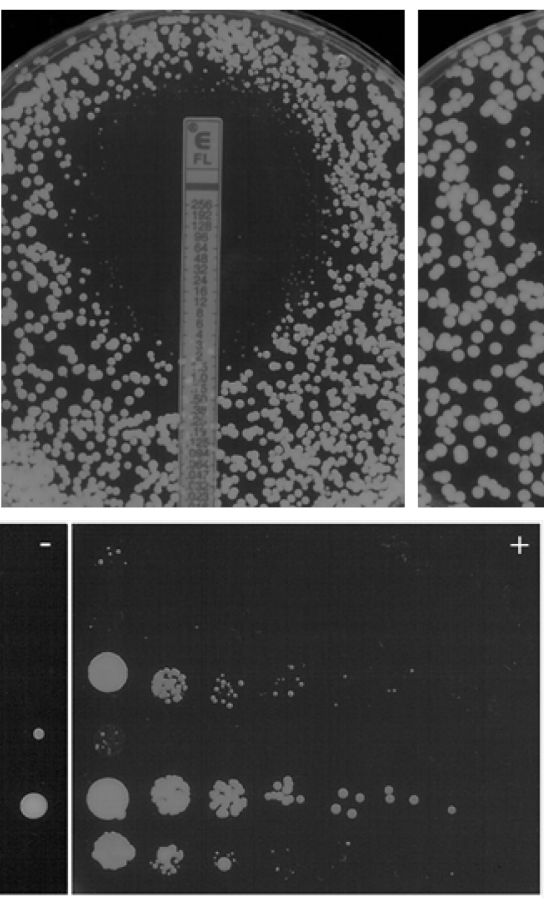

TACl ${ }^{\mathrm{G} 980 \mathrm{E}}$

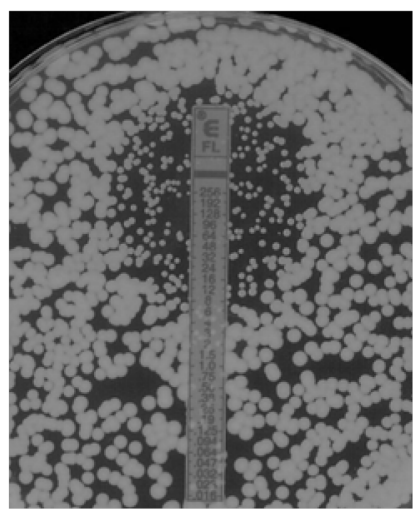

TAClcas $5 \Delta / \Delta$

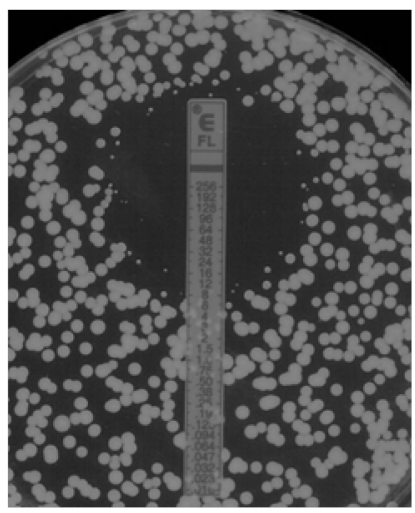

ERGII

ERGIlcas $5 \Delta / \Delta$

MRRI

MRRlcas $5 \Delta / \Delta$

TACI

TAClcas $5 \Delta / \Delta$

Figure 3-4. CAS5 slightly impacts fluconazole susceptibility in background of strains expressing resistance mechanisms

(A) Effect of CAS5 in isogenic resistant backgrounds on MIC and growth on YPD agar as determined by Etest. A confluent lawn of $C$. albicans was streaked prior to the addition of Etest strips and then incubated for 48 hours. (B) Effect of CAS5 on the ability of isogenic strains to grow on solid media containing fluconazole. From 4-fold serial dilutions of $C$. albicans strains, $2 \mu$ l aliquots were spotted onto YPD agar $+/-10$ $\mu \mathrm{g} / \mathrm{mL}$ FLC and incubated for 48 hours. 
fluconazole for the cas $5 \Delta / \Delta$ mutants compared to their respective backgrounds (Figure 3-4B).

\section{Expression of ERG11, CDR1, CDR2, and MDR1 When CAS5 Is Disrupted in Resistant Backgrounds}

One possible explanation for the enhanced fluconazole activity could be decreased expression of ERG11 or genes encoding the Cdr1 and Cdr2 or Mdr1 efflux pumps. In order to determine if this was the case, we measured ERG11, CDR1, CDR2, and $M D R 1$ mRNA abundance by qRT-PCR in the strains containing a single resistance mechanism and their respective cas $5 \Delta / \Delta$ mutants (Figure 3-5). Interestingly, the cas $5 \Delta / \Delta$ mutant constructed in the SC5314 background showed a slight reduction in baseline $E R G 11$ expression. This was also the case for the cas $5 \Delta / \Delta$ mutants constructed in the $E R G 11^{\mathrm{K} 143 \mathrm{R}}, M R R 1^{\mathrm{P} 683 \mathrm{~S}}, T A C 1^{\mathrm{G} 980 \mathrm{E}}$ and $U P C 2^{\mathrm{G} 648 \mathrm{D}}$ backgrounds. Disruption of $C A S 5$ did not result in decreased $C D R 1$ expression, except slightly in the $T A C 1^{\mathrm{G} 980 \mathrm{E}}$ background. Alternatively, disruption of $C A S 5$ increased $C D R 2$ expression in all backgrounds. Meanwhile $M D R 1$ expression also increased in the SC5314, ERG1 $1^{\mathrm{K} 143 \mathrm{R}}$, and $T A C 1^{\mathrm{G} 980 \mathrm{E}}$ backgrounds but decreased in the $M R R 1^{\mathrm{P} 683 \mathrm{~S}}$ background. The significance of the increased expression of these transporter genes is unclear. These data suggest that the enhanced activity of fluconazole observed in resistant strains lacking $C A S 5$ is not due to decreased expression of transporters gene expression levels. Instead, the observed phenotypes may be associated with a reduction in $E R G$ gene expression, particularly ERG11.

\section{Comparison of the Gene Expression Profiles of Wild-Type Strain SC5314 and $\operatorname{cas} 5 \Delta / \Delta$ Exposed to Fluconazole}

In order to identify genes whose expression in response to fluconazole is influenced by Cas5, we compared the transcriptional profiles of SC5314 and its cas $5 \Delta / \Delta$ derivative after treatment with or without $10 \mu \mathrm{g} / \mathrm{ml}$ fluconazole for 6 hours. Genes were considered to be differentially expressed in response to fluconazole if their expression changed by $\geq 1$.5-fold in two independent experiments (Table A-1 and Table A-2). Fluconazole inducible genes were also considered to be $C A S 5$-dependent if their induction was abrogated in the deletion mutant and was $\geq 2.0$-fold $(50 \%)$ less than compared to SC5314. Using these criteria, there were 209 genes upregulated by fluconazole whose induction was abrogated in the absence of CAS5 (Table 3-7). The most common biological processes represented by these genes include transport, response to chemical stimulus, oxidation-reduction process, filamentous growth, lipid metabolic process and cell wall organization. Interestingly, there were also 6 genes involved in iron ion transport, binding, and homeostasis. 

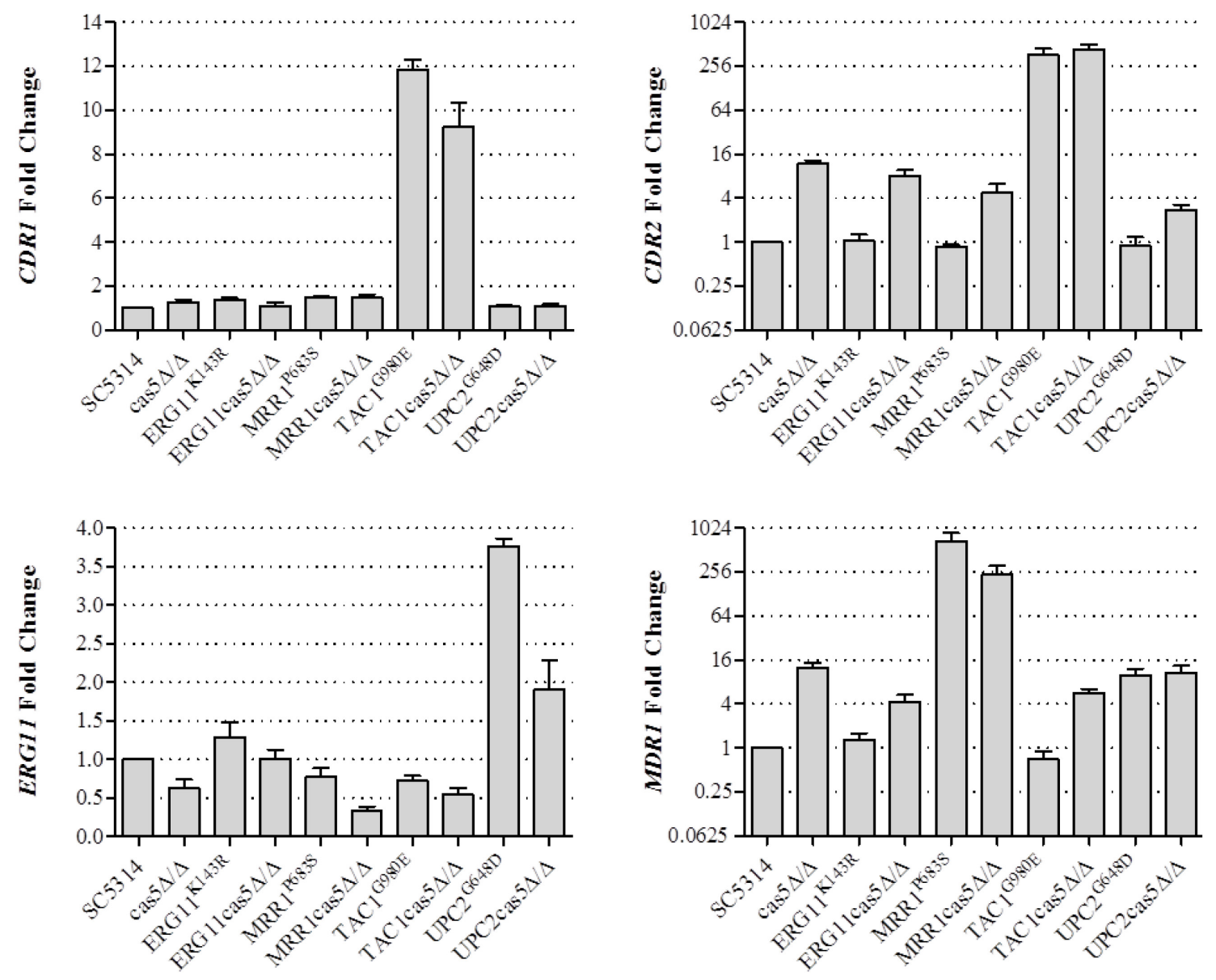

Figure 3-5. Expression of CDR1, CDR2, ERG11, and MDR1 by qRT-PCR Expression levels for $E R G 11, C D R 1, C D R 2$, and $M D R 1$ were measured in triplicate by qRT-PCR and compared to the expression levels in SC5314 in YPD. Error bars represent the standard error of the mean. 
Table 3-7. Genes upregulated by at least 1.5-fold by fluconazole which are dependent upon Cas5

\begin{tabular}{|c|c|c|c|c|c|c|c|c|}
\hline \multirow[b]{3}{*}{ Process $^{a}$} & \multirow[b]{3}{*}{ orf19 Designation } & \multirow[b]{3}{*}{ CGD Name } & \multicolumn{4}{|c|}{ Change in Fold Expression } & & \\
\hline & & & \multicolumn{2}{|c|}{$\begin{array}{c}\text { SC5314+FLC/ } \\
\text { SC5314 (A) } \\
\end{array}$} & \multicolumn{2}{|c|}{$\begin{array}{c}\operatorname{cas} 5 \Delta / \Delta+\mathrm{FLC} / \\
\operatorname{cas} 5 \Delta / \Delta(\mathrm{B}) \\
\end{array}$} & \multicolumn{2}{|c|}{ Ratio (B/A) } \\
\hline & & & Exp1 & Exp2 & $\operatorname{Exp} 1$ & Exp2 & Exp1 & $\operatorname{Exp2}$ \\
\hline \multirow{9}{*}{$\begin{array}{l}\text { Lipid metabolic } \\
\text { process }\end{array}$} & orf19.10 & $A L K 8$ & 9.0 & 8.1 & 3.0 & 3.5 & 0.3 & 0.4 \\
\hline & orf19.2248 & $A R E 2$ & 1.7 & 1.6 & 0.7 & 0.7 & 0.4 & 0.4 \\
\hline & orf19.2670 & & 1.6 & 1.8 & 0.4 & 0.4 & 0.3 & 0.2 \\
\hline & orf19.2909 & $E R G 26$ & 2.4 & 1.7 & 0.9 & 0.9 & 0.4 & 0.5 \\
\hline & orf19.4897 & SFH5 & 2.4 & 2.4 & 1.3 & 1.2 & 0.5 & 0.5 \\
\hline & orf19.4982 & & 2.1 & 2.3 & 0.9 & 0.6 & 0.4 & 0.3 \\
\hline & orf19.5640 & PEX5 & 2.2 & 2.6 & 0.9 & 0.9 & 0.4 & 0.3 \\
\hline & orf19.5751 & ORMI & 3.3 & 3.6 & 1.6 & 1.7 & 0.5 & 0.5 \\
\hline & orf19.6594 & $P L B 3$ & 2.1 & 3.4 & 1.1 & 0.9 & 0.5 & 0.3 \\
\hline \multirow{7}{*}{$\begin{array}{c}\text { Cell wall } \\
\text { organization }\end{array}$} & orf19.212 & VPS28 & 1.7 & 2.1 & 0.5 & 0.5 & 0.3 & 0.3 \\
\hline & orf19.5302 & $P G A 31$ & 17.6 & 4.1 & 2.1 & 1.5 & 0.1 & 0.4 \\
\hline & orf19.5644 & & 2.3 & 3.5 & 1.1 & 1.0 & 0.5 & 0.3 \\
\hline & orf19.6102 & $R C A 1$ & 2.5 & 3.4 & 1.2 & 1.2 & 0.5 & 0.4 \\
\hline & orf19.6420 & $P G A 13$ & 15.6 & 30.6 & 2.4 & 2.3 & 0.2 & 0.1 \\
\hline & orf19.6481 & YPS7 & 1.6 & 2.3 & 0.4 & 0.4 & 0.3 & 0.2 \\
\hline & orf19.719 & & 1.8 & 3.3 & 1.0 & 1.0 & 0.5 & 0.3 \\
\hline \multirow{3}{*}{$\begin{array}{c}\text { Cellular membrane } \\
\text { organization }\end{array}$} & orf19.3412 & $A T G 15$ & 2.0 & 2.6 & 0.7 & 1.0 & 0.4 & 0.4 \\
\hline & orf19.5644 & & 2.3 & 3.5 & 1.1 & 1.0 & 0.5 & 0.3 \\
\hline & orf19.669 & PRM1 & 2.2 & 2.8 & 0.6 & 0.4 & 0.3 & 0.1 \\
\hline \multirow[t]{2}{*}{ Iron ion transport } & orf19.1415 & FRE 10 & 2.0 & 4.2 & 0.9 & 2.3 & 0.5 & 0.5 \\
\hline & orf19.1932 & CFL4 & 35.6 & 359 & 2.4 & 6.6 & 0.1 & 0.0 \\
\hline
\end{tabular}


Table 3-7. (Continued)

\begin{tabular}{|c|c|c|c|c|c|c|c|c|}
\hline \multirow[b]{3}{*}{ Process $^{a}$} & \multirow[b]{3}{*}{ orf19 Designation } & \multirow[b]{3}{*}{ CGD Name } & \multicolumn{4}{|c|}{ Change in Fold Expression } & \multirow{2}{*}{\multicolumn{2}{|c|}{ Ratio (B/A) }} \\
\hline & & & \multicolumn{2}{|c|}{$\begin{array}{c}\text { SC5314+FLC/ } \\
\text { SC5314 (A) }\end{array}$} & \multicolumn{2}{|c|}{$\begin{array}{c}\operatorname{cas} 5 \Delta / \Delta+F L C / \\
\operatorname{cas} 5 \Delta / \Delta(B)\end{array}$} & & \\
\hline & & & Exp1 & Exp2 & Exp1 & Exp2 & Exp1 & Exp2 \\
\hline & orf19.4215 & FET34 & 2.1 & 5.2 & 0.2 & 0.5 & 0.1 & 0.1 \\
\hline & orf19.7219 & FTR1 & 3.2 & 7.8 & 0.5 & 1.1 & 0.1 & 0.1 \\
\hline Iron ion binding & orf19.1411 & & 2.7 & 4.8 & 1.1 & 0.9 & 0.4 & 0.2 \\
\hline Iron ion homeostasis & orf19.1264 & CFL2 & 2.0 & 6.0 & 0.6 & 0.5 & 0.3 & 0.1 \\
\hline \multirow[t]{18}{*}{ Transport } & orf19.111 & CAN2 & 1.7 & 13.0 & 0.7 & 1.5 & 0.4 & 0.1 \\
\hline & orf19.1252 & $Y M E 1$ & 1.9 & 3.0 & 1.0 & 0.7 & 0.5 & 0.2 \\
\hline & orf19.1313 & $C D R 3$ & 1.9 & 2.1 & 0.9 & 1.0 & 0.5 & 0.5 \\
\hline & orf19.1352 & TIM22 & 3.6 & 4.4 & 0.9 & 1.1 & 0.2 & 0.3 \\
\hline & orf19.1563 & ECM3 & 1.5 & 1.5 & 0.4 & 0.6 & 0.3 & 0.4 \\
\hline & orf19.1867 & & 2.6 & 4.2 & 0.7 & 0.6 & 0.3 & 0.2 \\
\hline & orf19.2073 & & 1.5 & 1.6 & 0.6 & 0.5 & 0.4 & 0.3 \\
\hline & orf19.2292 & OPT4 & 5.2 & 3.7 & 1.2 & 1.0 & 0.2 & 0.3 \\
\hline & orf19.2350 & & 2.5 & 4.1 & 0.6 & 1.0 & 0.3 & 0.3 \\
\hline & orf19.2810 & $A A P 1$ & 18.9 & 19.6 & 7.5 & 8.8 & 0.4 & 0.4 \\
\hline & orf19.2946 & HNM4 & 8.4 & 4.9 & 2.9 & 2.5 & 0.3 & 0.5 \\
\hline & orf19.3015 & $A R X 1$ & 2.7 & 3.9 & 1.3 & 1.7 & 0.5 & 0.4 \\
\hline & orf19.3195 & HIP1 & 1.5 & 2.6 & 0.6 & 0.7 & 0.4 & 0.3 \\
\hline & orf19.3232 & & 24.6 & 5.3 & 2.6 & 1.0 & 0.1 & 0.2 \\
\hline & orf19.3574 & MDJ2 & 5.6 & 3.1 & 2.0 & 1.2 & 0.4 & 0.4 \\
\hline & orf19.4041 & $P E X 4$ & 4.9 & 4.3 & 1.3 & 1.5 & 0.3 & 0.4 \\
\hline & orf19.4090 & & 2.1 & 1.7 & 1.1 & 0.8 & 0.5 & 0.5 \\
\hline & orf19.4372 & & 1.8 & 3.0 & 0.6 & 0.6 & 0.3 & 0.2 \\
\hline
\end{tabular}


Table 3-7. (Continued)

\begin{tabular}{|c|c|c|c|c|c|c|c|c|}
\hline \multirow[b]{3}{*}{ Process $^{\mathrm{a}}$} & \multirow[b]{3}{*}{ orf19 Designation } & \multirow[b]{3}{*}{ CGD Name } & \multicolumn{4}{|c|}{ Change in Fold Expression } & & \\
\hline & & & \multicolumn{2}{|c|}{$\begin{array}{c}\text { SC5314+FLC/ } \\
\text { SC5314 (A) }\end{array}$} & \multicolumn{2}{|c|}{$\begin{array}{c}\operatorname{cas} 5 \Delta / \Delta+\mathrm{FLC} / \\
\operatorname{cas} 5 \Delta / \Delta(\mathrm{B})\end{array}$} & \multicolumn{2}{|c|}{ Ratio (B/A) } \\
\hline & & & Exp1 & Exp2 & Exp1 & $\operatorname{Exp2}$ & Exp1 & Exp2 \\
\hline & orf19.4384 & $H X T 5$ & 70.6 & 75.5 & 11.1 & 23.4 & 0.2 & 0.3 \\
\hline & orf19.4546 & HOL4 & 4.3 & 5.1 & 1.7 & 2.3 & 0.4 & 0.4 \\
\hline & orf19.4682 & HGT17 & 45 & 24.7 & 7.0 & 7.3 & 0.2 & 0.3 \\
\hline & orf19.4887 & $E C M 21$ & 2.7 & 3.1 & 0.8 & 1.0 & 0.3 & 0.3 \\
\hline & orf19.5539 & & 1.6 & 1.6 & 0.7 & 0.8 & 0.5 & 0.5 \\
\hline & orf19.5902 & $R A S 2$ & 7.6 & 6.2 & 0.8 & 1.1 & 0.1 & 0.2 \\
\hline & orf19.5958 & $C D R 2$ & 2.1 & 2.5 & 0.9 & 1.0 & 0.4 & 0.4 \\
\hline & orf19.6117 & & 1.5 & 2.3 & 0.6 & 0.4 & 0.4 & 0.2 \\
\hline & orf19.6249 & $H A K 1$ & 4.4 & 10.7 & 2.1 & 3.2 & 0.5 & 0.3 \\
\hline & orf19.6648 & $S D A 1$ & 3.2 & 6.8 & 1.6 & 3.2 & 0.5 & 0.4 \\
\hline & orf19.6993 & $G A P 2$ & 21.7 & 8.6 & 3.0 & 4.5 & 0.1 & 0.5 \\
\hline & orf19.7056 & & 6.2 & 120.3 & 2.5 & 2.7 & 0.4 & 0.0 \\
\hline & orf19.7093 & $H G T 13$ & 30.7 & 13.2 & 2.0 & 1.5 & 0.1 & 0.1 \\
\hline & orf19.7094 & HGT12 & 423.2 & 19.8 & 8.0 & 1.9 & 0.0 & 0.1 \\
\hline \multirow[t]{5}{*}{ Response to stress } & orf19.1434 & & 1.7 & 2.7 & 0.8 & 1.0 & 0.5 & 0.4 \\
\hline & orf19.211 & & 1.7 & 2.4 & 0.7 & 0.7 & 0.4 & 0.3 \\
\hline & orf19.3239 & CTF 18 & 2.7 & 2.4 & 1.0 & 1.1 & 0.4 & 0.5 \\
\hline & orf19.4383 & & 2.8 & 2.6 & 0.9 & 0.8 & 0.3 & 0.3 \\
\hline & orf19.5069 & & 14.9 & 15.5 & 5.9 & 7.1 & 0.4 & 0.5 \\
\hline \multirow{4}{*}{$\begin{array}{l}\text { Response to } \\
\text { chemical stimulus }\end{array}$} & orf19.2060 & SOD5 & 33.2 & 14.2 & 5.4 & 6.1 & 0.2 & 0.4 \\
\hline & orf19.2838 & & 2.5 & 2.9 & 0.8 & 0.7 & 0.3 & 0.3 \\
\hline & orf19.2876 & $C B F 1$ & 2.3 & 3.2 & 0.5 & 0.5 & 0.2 & 0.2 \\
\hline & orf19.3159 & UTP20 & 3.8 & 8.9 & 1.9 & 3.5 & 0.5 & 0.4 \\
\hline
\end{tabular}


Table 3-7. (Continued)

\begin{tabular}{|c|c|c|c|c|c|c|c|c|}
\hline \multirow[b]{3}{*}{ Process $^{a}$} & \multirow[b]{3}{*}{ orf19 Designation } & \multirow[b]{3}{*}{ CGD Name } & \multicolumn{4}{|c|}{ Change in Fold Expression } & & \\
\hline & & & \multicolumn{2}{|c|}{$\begin{array}{c}\text { SC5314+FLC/ } \\
\text { SC5314 (A) }\end{array}$} & \multicolumn{2}{|c|}{$\begin{array}{c}\operatorname{cas} 5 \Delta / \Delta+F L C / \\
\operatorname{cas} 5 \Delta / \Delta(\mathrm{B})\end{array}$} & \multicolumn{2}{|c|}{ Ratio (B/A) } \\
\hline & & & Exp1 & $\operatorname{Exp2}$ & Exp1 & Exp2 & Exp1 & Exp2 \\
\hline & orf19.3736 & $K A R 4$ & 2.8 & 2.6 & 1.2 & 1.0 & 0.4 & 0.4 \\
\hline & orf19.4015 & $C A G 1$ & 3.1 & 3.6 & 0.8 & 0.7 & 0.3 & 0.2 \\
\hline & orf19.4317 & GRE3 & 1.5 & 1.5 & 0.8 & 0.6 & 0.5 & 0.4 \\
\hline & orf19.4318 & $M I G 1$ & 1.7 & 2.1 & 0.7 & 1.0 & 0.4 & 0.5 \\
\hline & orf19.5326 & & 1.5 & 1.9 & 0.6 & 0.7 & 0.4 & 0.4 \\
\hline & orf19.5591 & $A D O 1$ & 2.4 & 2.9 & 1.1 & 1.5 & 0.4 & 0.5 \\
\hline & orf19.6202 & RBT4 & 3.3 & 6.2 & 1.6 & 2.2 & 0.5 & 0.3 \\
\hline & orf19.6881 & $Y T H 1$ & 1.9 & 2.0 & 0.9 & 0.9 & 0.4 & 0.4 \\
\hline & orf19.7316 & & 2.0 & 2.3 & 0.7 & 0.8 & 0.3 & 0.3 \\
\hline & orf19.7384 & NOG1 & 3.7 & 7.2 & 1.2 & 2.1 & 0.3 & 0.3 \\
\hline Oxidation-reduction & orf19.1117 & & 5.7 & 2.3 & 0.7 & 1.1 & 0.1 & 0.5 \\
\hline process & orf19.1473 & & 35.0 & 15.0 & 3.8 & 4.8 & 0.1 & 0.3 \\
\hline & orf19.2108 & SOD6 & 7.9 & 10.6 & 1.6 & 1.1 & 0.2 & 0.1 \\
\hline & orf19.3538 & FRE9 & 4.7 & 14.9 & 2.5 & 3.6 & 0.5 & 0.2 \\
\hline & orf19.3707 & $Y H B 1$ & 3.0 & 2.8 & 0.9 & 0.6 & 0.3 & 0.2 \\
\hline & orf19.4747 & HEMI4 & 1.7 & 2.0 & 0.6 & 0.4 & 0.3 & 0.2 \\
\hline & orf19.4871 & ERO1 & 1.9 & 1.9 & 0.9 & 0.6 & 0.5 & 0.3 \\
\hline & orf19.5879 & & 3.3 & 2.9 & 1.5 & 1.2 & 0.5 & 0.4 \\
\hline & orf19.638 & $F D H 1$ & 11.7 & 19.5 & 4.4 & 3.9 & 0.4 & 0.2 \\
\hline & orf19.6837 & $F M A 1$ & 1.5 & 1.5 & 0.7 & 0.7 & 0.5 & 0.4 \\
\hline & orf19.7111.1 & SOD3 & 40.5 & 69.2 & 9.4 & 15 & 0.2 & 0.2 \\
\hline & orf19.7314 & $C D G 1$ & 7.5 & 6.0 & 1.0 & 0.6 & 0.1 & 0.1 \\
\hline & orf19.742 & $A L D 6$ & 11.5 & 10.4 & 4.1 & 1.7 & 0.4 & 0.2 \\
\hline & orf19.7551 & $A L O 1$ & 1.5 & 2.8 & 0.7 & 0.6 & 0.5 & 0.2 \\
\hline
\end{tabular}


Table 3-7. (Continued)

\begin{tabular}{|c|c|c|c|c|c|c|c|c|}
\hline \multirow[b]{3}{*}{ Process $^{a}$} & \multirow[b]{3}{*}{ orf19 Designation } & \multirow[b]{3}{*}{ CGD Name } & \multicolumn{4}{|c|}{ Change in Fold Expression } & & \\
\hline & & & \multicolumn{2}{|c|}{$\begin{array}{c}\text { SC5314+FLC/ } \\
\text { SC5314 (A) }\end{array}$} & \multicolumn{2}{|c|}{$\begin{array}{c}\operatorname{cas} 5 \Delta / \Delta+F L C / \\
\operatorname{cas} 5 \Delta / \Delta(\mathrm{B})\end{array}$} & \multicolumn{2}{|c|}{ Ratio (B/A) } \\
\hline & & & Exp1 & Exp2 & Exp1 & Exp2 & Exp1 & $\operatorname{Exp2}$ \\
\hline \multirow{12}{*}{ Filamentous growth } & orf19.2397.3 & & 2.0 & 4.3 & 0.9 & 1.2 & 0.5 & 0.3 \\
\hline & orf19.4055 & & 1.9 & 2.5 & 0.9 & 0.7 & 0.5 & 0.3 \\
\hline & orf19.4815 & YTM1 & 2.3 & 3.2 & 0.6 & 1.4 & 0.3 & 0.4 \\
\hline & orf19.4928 & SEC2 & 2.0 & 2.1 & 0.4 & 0.4 & 0.2 & 0.2 \\
\hline & orf19.5741 & ALS1 & 66.8 & 79.0 & 22.6 & 21 & 0.3 & 0.3 \\
\hline & orf19.5798 & $L I G 4$ & 1.8 & 2.6 & 0.9 & 0.8 & 0.5 & 0.3 \\
\hline & orf19.6595 & $R T A 4$ & 10.4 & $15 . .0$ & 3.0 & 2.9 & 0.3 & 0.2 \\
\hline & orf19.6888 & & 4.3 & 5.7 & 1.4 & 1.1 & 0.3 & 0.2 \\
\hline & orf19.7313 & SSU1 & 4.5 & 4.8 & 1.3 & 1.1 & 0.3 & 0.2 \\
\hline & orf19.7374 & CTA4 & 1.7 & 2.2 & 0.8 & 1.1 & 0.5 & 0.5 \\
\hline & orf19.7436 & $A A F 1$ & 2.0 & 4.4 & 1.0 & 1.2 & 0.5 & 0.3 \\
\hline & orf19.795 & VPS36 & 2.0 & 2.5 & 0.6 & 0.6 & 0.3 & 0.3 \\
\hline Biofilm formation & orf19.6090 & & 1.9 & 2.4 & 0.6 & 0.6 & 0.3 & 0.2 \\
\hline \multirow[t]{3}{*}{ Pathogenesis } & orf19.5542 & SAP6 & 5.9 & 4.3 & 2.1 & 1.4 & 0.4 & 0.3 \\
\hline & orf19.5585 & SAP5 & 3.7 & 3.0 & 0.9 & 1.0 & 0.2 & 0.3 \\
\hline & orf19.5716 & SAP4 & 4.8 & 3.2 & 2.1 & 1.5 & 0.4 & 0.5 \\
\hline \multirow{3}{*}{$\begin{array}{c}\text { Organelle } \\
\text { organization }\end{array}$} & orf19.3135 & & 2.3 & 2.6 & 1.2 & 1.1 & 0.5 & 0.4 \\
\hline & orf19.4862.2 & PET100 & 1.7 & 1.8 & 0.8 & 0.9 & 0.5 & 0.5 \\
\hline & orf19.6225.1 & & 1.9 & 1.9 & 0.9 & 0.7 & 0.5 & 0.4 \\
\hline \multirow{2}{*}{$\begin{array}{l}\text { RNA metabolic } \\
\text { process }\end{array}$} & orf19.1915 & MPP10 & 3.8 & 4.5 & 2.1 & 2.5 & 0.5 & 0.5 \\
\hline & orf19.1923 & $R R N 3$ & 5.7 & 23.0 & 2.9 & 3.8 & 0.5 & 0.2 \\
\hline
\end{tabular}


Table 3-7. (Continued)

\begin{tabular}{|c|c|c|c|c|c|c|c|c|}
\hline \multirow[b]{3}{*}{ Process $^{a}$} & \multirow[b]{3}{*}{ orf19 Designation } & \multirow[b]{3}{*}{ CGD Name } & \multicolumn{4}{|c|}{ Change in Fold Expression } & \multirow{2}{*}{\multicolumn{2}{|c|}{ Ratio (B/A) }} \\
\hline & & & \multicolumn{2}{|c|}{$\begin{array}{c}\text { SC5314+FLC/ } \\
\text { SC5314 (A) }\end{array}$} & \multicolumn{2}{|c|}{$\begin{array}{c}\operatorname{cas} 5 \Delta / \Delta+F L C / \\
\operatorname{cas} 5 \Delta / \Delta(\mathrm{B})\end{array}$} & & \\
\hline & & & Exp1 & Exp2 & Exp1 & Exp2 & Exp1 & Exp2 \\
\hline \multirow{13}{*}{ Biological process } & orf19.2115 & & 1.9 & 2.8 & 0.9 & 1.5 & 0.5 & 0.5 \\
\hline & orf19.2137 & & 1.9 & 2.3 & 0.6 & 0.8 & 0.3 & 0.4 \\
\hline & orf19.3962 & $H A S 1$ & 1.7 & 2.3 & 0.7 & 0.8 & 0.4 & 0.4 \\
\hline & orf19.501 & & 2.4 & 3.3 & 1.0 & 1.4 & 0.4 & 0.4 \\
\hline & orf19.5614 & & 7.9 & 7.7 & 3.9 & 4.1 & 0.5 & 0.5 \\
\hline & orf19.7062 & $R P A 135$ & 2.0 & 5.6 & 0.9 & 1.6 & 0.5 & 0.3 \\
\hline & orf19.2064 & & 1.9 & 3.0 & 0.9 & 0.8 & 0.5 & 0.3 \\
\hline & orf19.2242 & PRB1 & 1.7 & 1.5 & 0.7 & 0.6 & 0.4 & 0.4 \\
\hline & orf19.2710 & & 2.6 & 1.8 & 1.0 & 0.9 & 0.4 & 0.5 \\
\hline & orf19.3547 & & 5.3 & 5.9 & 1.1 & 1.7 & 0.2 & 0.3 \\
\hline & orf19.467 & WOR3 & 32.3 & 7.1 & 3.0 & 2.9 & 0.1 & 0.4 \\
\hline & orf19.5045 & PTP2 & 6.0 & 10.4 & 2.5 & 2.0 & 0.4 & 0.2 \\
\hline & orf19.6225 & PCL7 & 1.5 & 2.0 & 0.8 & 0.8 & 0.5 & 0.4 \\
\hline \multirow{3}{*}{$\begin{array}{l}\text { Cellular protein } \\
\text { modification }\end{array}$} & orf19.5236 & & 1.7 & 2.2 & 0.5 & 0.4 & 0.3 & 0.2 \\
\hline & orf19.540 & & 1.7 & 1.7 & 0.9 & 0.6 & 0.5 & 0.4 \\
\hline & orf19.7547 & & 2.9 & 3.0 & 1.1 & 1.4 & 0.4 & 0.5 \\
\hline \multirow{3}{*}{$\begin{array}{c}\text { Carbohydrate } \\
\text { metabolic process }\end{array}$} & orf19.4899 & $G C A 1$ & 4.8 & 4.4 & 0.9 & 1.2 & 0.2 & 0.3 \\
\hline & orf19.7434 & $G L G 2$ & 37.5 & 16.1 & 7.5 & 8.7 & 0.2 & 0.5 \\
\hline & orf19.7514 & $P C K 1$ & 6.0 & 8.6 & 1.9 & 1.4 & 0.3 & 0.2 \\
\hline \multirow[t]{2}{*}{ Other } & orf19.1369 & & 2.9 & 2.3 & 1.2 & 1.0 & 0.4 & 0.4 \\
\hline & orf19.2046 & POT1-2 & 1.5 & 2.2 & 0.5 & 0.4 & 0.3 & 0.2 \\
\hline
\end{tabular}


Table 3-7. (Continued)

\begin{tabular}{|c|c|c|c|c|c|c|c|c|}
\hline \multirow[b]{3}{*}{ Process $^{a}$} & \multirow[b]{3}{*}{ orf19 Designation } & \multirow[b]{3}{*}{ CGD Name } & \multicolumn{4}{|c|}{ Change in Fold Expression } & \multirow{2}{*}{\multicolumn{2}{|c|}{ Ratio (B/A) }} \\
\hline & & & \multicolumn{2}{|c|}{$\begin{array}{c}\text { SC5314+FLC/ } \\
\text { SC5314 (A) }\end{array}$} & \multicolumn{2}{|c|}{$\begin{array}{c}\operatorname{cas} 5 \Delta / \Delta+F L C / \\
\operatorname{cas} 5 \Delta / \Delta(\mathrm{B})\end{array}$} & & \\
\hline & & & Exp1 & Exp2 & Exp1 & Exp2 & Exp1 & Exp2 \\
\hline & orf19.278 & & 2.5 & 2.7 & 1.4 & 1.5 & 0.5 & 0.5 \\
\hline & orf19.410.3 & RIB4 & 5.6 & 5.7 & 0.6 & 0.5 & 0.1 & 0.1 \\
\hline & orf19.4551 & CTN1 & 22.4 & 26.6 & 11.3 & 14.3 & 0.5 & 0.5 \\
\hline & orf19.5159 & $D U G 3$ & 1.8 & 3.6 & 0.9 & 0.7 & 0.5 & 0.2 \\
\hline & orf19.5206 & & 5.2 & 4.0 & 1.4 & 1.7 & 0.3 & 0.4 \\
\hline & orf19.5454 & $D A L 1$ & 3.7 & 6.6 & 1.7 & 1.9 & 0.5 & 0.3 \\
\hline & orf19.6057 & & 2.5 & 1.8 & 0.9 & 1.0 & 0.3 & 0.5 \\
\hline & orf19.6997 & ATO5 & 2.5 & 2.6 & 0.8 & 0.7 & 0.3 & 0.3 \\
\hline & orf19.972 & & 1.9 & 2.9 & 0.8 & 0.6 & 0.4 & 0.2 \\
\hline \multirow[t]{15}{*}{ Unknown } & orf19.1277 & & 6.6 & 9.3 & 1.5 & 1.5 & 0.2 & 0.2 \\
\hline & orf19.1766 & & 26.6 & 340.0 & 3.3 & 5.7 & 0.1 & 0.0 \\
\hline & orf19.1800 & & 4.1 & 3.8 & 2.1 & 1.8 & 0.5 & 0.5 \\
\hline & orf19.1939 & & 2.3 & 1.6 & 0.6 & 0.8 & 0.3 & 0.5 \\
\hline & orf19.2059 & & 2.6 & 2.3 & 0.2 & 0.3 & 0.1 & 0.1 \\
\hline & orf19.2210 & & 2.3 & 4.3 & 1.0 & 1.9 & 0.4 & 0.5 \\
\hline & orf19.2398 & & 2.5 & 3.8 & 1.3 & 1.7 & 0.5 & 0.5 \\
\hline & orf19.2414 & & 3.1 & 3.3 & 1.1 & 0.6 & 0.4 & 0.2 \\
\hline & orf19.2691 & & 1.5 & 1.5 & 0.5 & 0.7 & 0.3 & 0.4 \\
\hline & orf19.2812 & & 1.8 & 1.8 & 0.8 & 1.0 & 0.4 & 0.5 \\
\hline & orf19.2836 & & 11.7 & 21.9 & 3.1 & 4.8 & 0.3 & 0.2 \\
\hline & orf19.2839 & CIRT4B & 1.6 & 2.6 & 0.4 & 0.3 & 0.2 & 0.1 \\
\hline & orf19.2878 & $P G A 15$ & 6.0 & 8.1 & 3.1 & 2.4 & 0.5 & 0.3 \\
\hline & orf19.296 & & 3.1 & 1.7 & 1.5 & 0.9 & 0.5 & 0.5 \\
\hline & orf19.2962 & & 2.3 & 2.2 & 0.7 & 0.8 & 0.3 & 0.4 \\
\hline
\end{tabular}


Table 3-7. (Continued)

\begin{tabular}{|c|c|c|c|c|c|c|c|c|}
\hline \multirow[b]{3}{*}{ Process $^{a}$} & \multirow[b]{3}{*}{ orf19 Designation } & \multirow[b]{3}{*}{ CGD Name } & \multicolumn{4}{|c|}{ Change in Fold Expression } & & \\
\hline & & & \multicolumn{2}{|c|}{$\begin{array}{c}\text { SC5314+FLC/ } \\
\text { SC5314 (A) }\end{array}$} & \multicolumn{2}{|c|}{$\begin{array}{c}\operatorname{cas} 5 \Delta / \Delta+F L C / \\
\operatorname{cas} 5 \Delta / \Delta(\mathrm{B})\end{array}$} & \multicolumn{2}{|c|}{ Ratio (B/A) } \\
\hline & & & Exp1 & Exp2 & Exp1 & $\operatorname{Exp2}$ & Exp1 & Exp2 \\
\hline & orf19.3004 & & 1.5 & 2.0 & 0.6 & 0.6 & 0.4 & 0.3 \\
\hline & orf19.3021 & & 2.6 & 2.0 & 1.2 & 0.9 & 0.5 & 0.5 \\
\hline & orf19.3245 & & 2.2 & 2.3 & 1.2 & 1.1 & 0.5 & 0.5 \\
\hline & orf19.3302 & & 1.5 & 2.1 & 0.7 & 0.8 & 0.5 & 0.4 \\
\hline & orf19.3309 & & 1.5 & 1.7 & 0.8 & 0.6 & 0.5 & 0.3 \\
\hline & orf19.3310 & & 1.8 & 1.9 & 0.8 & 0.6 & 0.4 & 0.3 \\
\hline & orf19.3499 & & 79.5 & 20.5 & 5.9 & 7.6 & 0.1 & 0.4 \\
\hline & orf19.3512 & CSP1 & 2.6 & 2.8 & 0.9 & 1.2 & 0.4 & 0.4 \\
\hline & orf19.3621 & & 3.2 & 5.2 & 0.7 & 0.7 & 0.2 & 0.1 \\
\hline & orf19.376 & & 2.9 & 3.4 & 1.1 & 1.5 & 0.4 & 0.4 \\
\hline & orf19.3881 & & 1.9 & 2.0 & 0.7 & 0.6 & 0.4 & 0.3 \\
\hline & orf19.3910 & & 3.1 & 2.6 & 1.1 & 0.9 & 0.4 & 0.3 \\
\hline & orf19.4013 & & 2.6 & 2.2 & 0.6 & 0.6 & 0.2 & 0.3 \\
\hline & orf19.4014 & & 1.9 & 1.6 & 0.8 & 0.8 & 0.4 & 0.5 \\
\hline & orf19.4144 & & 2.4 & 3.2 & 1.2 & 1.6 & 0.5 & 0.5 \\
\hline & orf19.4214 & & 30.5 & 19.5 & 0.5 & 1.2 & 0.0 & 0.1 \\
\hline & orf19.4264 & & 2.5 & 3.2 & 1.2 & 1.0 & 0.5 & 0.3 \\
\hline & orf19.4286 & & 1.9 & 2.9 & 0.3 & 0.4 & 0.2 & 0.1 \\
\hline & orf19.4404 & PGA49 & 7.3 & 1.6 & 1.1 & 0.8 & 0.2 & 0.5 \\
\hline & orf19.4450.1 & & 3.6 & 4.5 & 1.0 & 0.7 & 0.3 & 0.2 \\
\hline & orf19.4539 & & 1.8 & 1.7 & 0.9 & 0.5 & 0.5 & 0.3 \\
\hline & orf19.4569 & & 1.8 & 2.0 & 0.8 & 0.8 & 0.4 & 0.4 \\
\hline & orf19.4596 & & 3.5 & 4.9 & 1.0 & 1.1 & 0.3 & 0.2 \\
\hline & orf19.4607 & & 5.6 & 7.0 & 0.5 & 0.3 & 0.1 & 0.0 \\
\hline & orf19.4608 & $P D C 12$ & 7.1 & 16.7 & 0.5 & 0.3 & 0.1 & 0.0 \\
\hline
\end{tabular}


Table 3-7. (Continued)

\begin{tabular}{|c|c|c|c|c|c|c|c|c|}
\hline \multirow[b]{3}{*}{ Process $^{\mathrm{a}}$} & \multirow[b]{3}{*}{ orf19 Designation } & \multirow[b]{3}{*}{ CGD Name } & \multicolumn{4}{|c|}{ Change in Fold Expression } & & \\
\hline & & & \multicolumn{2}{|c|}{$\begin{array}{c}\text { SC5314+FLC/ } \\
\text { SC5314 (A) }\end{array}$} & \multicolumn{2}{|c|}{$\begin{array}{c}\operatorname{cas} 5 \Delta / \Delta+F L C / \\
\operatorname{cas} 5 \Delta / \Delta(\mathrm{B})\end{array}$} & \multicolumn{2}{|c|}{ Ratio (B/A) } \\
\hline & & & Exp1 & Exp2 & Exp1 & $\operatorname{Exp2}$ & Exp1 & Exp2 \\
\hline & orf19.4658 & & 5.0 & 6.6 & 2.5 & 3.1 & 0.5 & 0.5 \\
\hline & orf19.4691 & & 16.6 & 24.8 & 4.2 & 3.4 & 0.3 & 0.1 \\
\hline & orf19.4872 & & 8.2 & 19.2 & 1.2 & 1.3 & 0.1 & 0.1 \\
\hline & orf19.5019 & & 2.8 & 3.4 & 1.4 & 1.8 & 0.5 & 0.5 \\
\hline & orf19.5282 & & 2.4 & 2.7 & 0.5 & 0.4 & 0.2 & 0.2 \\
\hline & orf19.5626 & & 3.6 & 4.0 & 1.5 & 1.4 & 0.4 & 0.3 \\
\hline & orf19.6084 & & 123.7 & 19.7 & 11.8 & 9.8 & 0.1 & 0.5 \\
\hline & orf19.6186 & & 12.1 & 13.3 & 2.4 & 2.0 & 0.2 & 0.1 \\
\hline & orf19.6248 & & 3.1 & 12.1 & 0.7 & 1.2 & 0.2 & 0.1 \\
\hline & orf19.633 & & 1.5 & 1.6 & 0.8 & 0.5 & 0.5 & 0.3 \\
\hline & orf19.6353 & & 1.8 & 2.0 & 0.7 & 0.6 & 0.4 & 0.3 \\
\hline & orf19.6484 & & 19.5 & 30.0 & 2.2 & 4.3 & 0.1 & 0.1 \\
\hline & orf19.6527 & & 1.5 & 1.8 & 0.8 & 0.7 & 0.5 & 0.4 \\
\hline & orf19.6678 & & 3.1 & 5.1 & 1.2 & 1.4 & 0.4 & 0.3 \\
\hline & orf19.6690 & & 2.4 & 2.3 & 0.7 & 0.6 & 0.3 & 0.3 \\
\hline & orf19.670.2 & & 47.4 & 152.7 & 10.8 & 16.1 & 0.2 & 0.1 \\
\hline & orf19.6766 & NOP13 & 5.1 & 5.3 & 1.7 & 2.6 & 0.3 & 0.5 \\
\hline & orf19.6905 & & 2.4 & 3.3 & 0.5 & 0.4 & 0.2 & 0.1 \\
\hline & orf19.6970 & & 4.5 & 4.4 & 2.3 & 2.4 & 0.5 & 0.5 \\
\hline & orf19.6983 & & 2.1 & 9.1 & 1.0 & 1.3 & 0.5 & 0.1 \\
\hline & orf19.7011 & & 2.2 & 4.7 & 1.1 & 2.3 & 0.5 & 0.5 \\
\hline & orf19.7043 & & 3.9 & 2.8 & 0.9 & 1.0 & 0.2 & 0.4 \\
\hline & orf19.7229 & $I M L 2$ & 2.8 & 3.3 & 1.4 & 1.4 & 0.5 & 0.4 \\
\hline & orf19.7550 & IFA14 & 2.3 & 1.7 & 1.0 & 0.7 & 0.4 & 0.4 \\
\hline & orf19.7567 & & 2.5 & 2.4 & 1.1 & 0.8 & 0.4 & 0.3 \\
\hline
\end{tabular}


Table 3-7. (Continued)

\begin{tabular}{|c|c|c|c|c|c|c|c|c|}
\hline \multirow[b]{3}{*}{ Process $^{a}$} & \multirow[b]{3}{*}{ orf19 Designation } & \multirow[b]{3}{*}{ CGD Name } & \multicolumn{4}{|c|}{ Change in Fold Expression } & & \\
\hline & & & \multicolumn{2}{|c|}{$\begin{array}{c}\text { SC5314+FLC/ } \\
\text { SC5314 (A) }\end{array}$} & \multicolumn{2}{|c|}{$\begin{array}{c}\operatorname{cas} 5 \Delta / \Delta+F L C / \\
\operatorname{cas} 5 \Delta / \Delta(\mathrm{B})\end{array}$} & \multicolumn{2}{|c|}{ Ratio (B/A) } \\
\hline & & & Exp1 & Exp2 & Exp1 & Exp2 & Exp1 & $\operatorname{Exp2}$ \\
\hline & orf19.893 & $P G A 17$ & 12.3 & 5.5 & 1.4 & 1.1 & 0.1 & 0.2 \\
\hline & orf19.951 & & 2.3 & 2.4 & 0.4 & 0.4 & 0.2 & 0.2 \\
\hline & orf19.994 & & 2.0 & 2.7 & 0.7 & 1.3 & 0.4 & 0.5 \\
\hline
\end{tabular}

${ }^{a}$ Descriptions are from the Candida Genome Database (http://www.candidagenome.org). 


\section{Validation of Microarray Data by Real Time RT-PCR}

In order to validate the differential expression of genes identified by microarray, we examined the mRNA abundance for 5 genes of interest using the same RNA isolated for the microarray experiments. PGA13 was chosen because it is involved in cell wall organization and was also identified by Bruno et al. to be Cas5-dependent (134). In addition, four others were chosen based on their involvement in cell wall organization (PGA31), iron ion transport (CFL4, FTR1) and lipid metabolic process (ERG26). The correlation between the microarray data and the real time RT-PCR was good (Figure 3-6). The expressions of CFL4, ERG26, FTR1, PGA13, and PGA31 were upregulated in the wild type SC5314 when treated with fluconazole, but could not respond to the same extent when $C A S 5$ was disrupted.

\section{DISCUSSION}

Identifying transcriptional networks that are central to azole antifungal susceptibility would be instructive for developing co-therapeutic strategies that would enhance their activity against $C$. albicans. Several signal transduction and transcriptional activation programs have been identified to respond to antifungal stress, but the transcription factors and their respective target genes have yet to be identified for many of these processes $(98-102,105,236,237)$. In the present study, we screened a library of C. albicans mutants disrupted for genes encoding TFs in order to identify those which exhibit increased susceptibility to fluconazole. We observed that disruption of CAS5 resulted in a slightly enhanced MIC, but also resulted in a substantial reduction in fluconazole MFC at 24, 48, and 72 hours. Indeed disruption of CAS5 in an azole susceptible strain prevented its regrowth in YPD medium in the presence of higher fluconazole concentrations after 72 hours, resulted in a clear zone of inhibition around a fluconazole Etest strip, and prevented growth on solid media containing a therapeutically relevant concentration of fluconazole $(10 \mu \mathrm{g} / \mathrm{ml})$. Time-kill analysis also demonstrated an enhanced effect of $10 \mu \mathrm{g} / \mathrm{ml}$ fluconazole against the cas $5 \Delta / \Delta$ mutant as compared to its parent strain. The disruption of RPN4 did not display as strong of an effect, suggesting that $R P N 4$ influences fungistatic azole activity to a lesser extent, while $C Z F 1$ disruption had no impact on susceptibility. As previously observed for disruption of $U P C 2$ (Chapter 2), CAS5 disruption strongly impacts fluconazole susceptibility.

We then sought to determine if disruption of $M K C 1$, involved in the CWI pathway, would have the same impact on fluconazole susceptibility as seen upon disruption of $C A S 5$, as Cas 5 has been postulated to be the transcriptional regulator of the CWI pathway (134). We observed that disruption of $M K C 1$ did have an effect on fluconazole susceptibility, but not as pronounced as was observed for disruption CAS5. Disruption of $M K C 1$ did result in a clear zone of inhibition around a fluconazole Etest strip, prevented its regrowth in YPD medium in the presence of higher fluconazole concentrations after 72 hours, and prevented growth on solid media containing a therapeutically relevant concentration of fluconazole $(10 \mu \mathrm{g} / \mathrm{ml})$. Time-kill analysis also demonstrated a slightly enhanced effect of $10 \mu \mathrm{g} / \mathrm{ml}$ fluconazole against the $m \mathrm{kcl} 1 \Delta / \Delta$ 


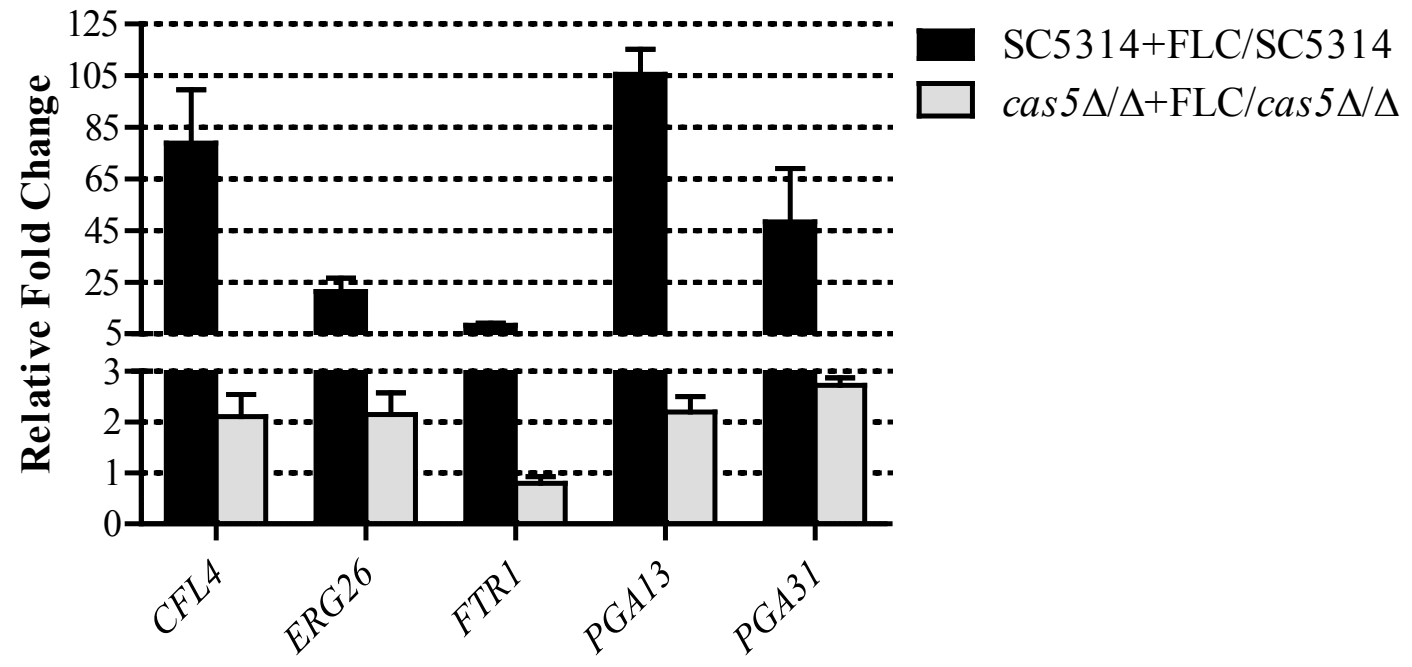

Figure 3-6. Validation of fluconazole-inducible and Cas5-dependent gene expression by qRT-PCR

Expression levels for CFL4, ERG26, FTR1, PGA13 and PGA31 were measured in triplicate by qRT-PCR and compared to the expression levels in SC5314 in YPD. Shown are the relative $n$-fold changes in gene expression in the in SC5314 and cas $5 \Delta / \Delta$ treated with fluconazole (FLC). Error bars represent the standard error of the mean. 
mutant compared to its parent strain, but was still able to grow above its starting inoculum. This suggests that $M K C 1$ plays a smaller role in fluconazole susceptibility and Cas $5 p$ may represent a different or redundant pathway.

Our observations for $M K C 1$ are similar to those of others. Epp et al. observed a reduction in fluconazole MIC from $2 \mu \mathrm{g} / \mathrm{ml}$ to $1 \mu \mathrm{g} / \mathrm{ml}$ at 24 hours, $>128 \mu \mathrm{g} / \mathrm{ml}$ to 2 $\mu \mathrm{g} / \mathrm{ml}$ at 48 hours, but after 72 hours both its parent and the $m k c 1 \Delta / \Delta$ mutant had an MIC of $>128 \mu \mathrm{g} / \mathrm{ml}$. This trend of regrowth after extended time periods was also seen when plated on YPD plates containing $2 \mu \mathrm{g} / \mathrm{ml}$ fluconazole. After 2 days (48 hours) the $m k c 1 \Delta / \Delta$ mutant was unable to grow in the presence of fluconazole compared to the parent strain, but after 6 days the mutant showed signs of growth (244). LaFayette et al. also observed a decrease in ergosterol biosynthesis inhibitor MICs for $m k c l \Delta / \Delta$ but its 48 hour MFC was comparable to wild type (106). Taken together, these findings suggest a partial role for Mkclp in the susceptibility of $C$. albicans to the azoles.

Upon disruption of $C A S 5$ in fluconazole resistant clinical isolates, 12-99, G5, Gu5, and S2, which carry combinations of the four most common mechanisms of resistance, there was no marked change in susceptibility, except slightly in the G5 background. The disruption of CAS5 in isogenic strains containing specific resistance mutations in ERG11, MRR1, or TAC1, resulted in slightly increased susceptibility. However, he strain containing the $U P C 2^{\mathrm{G} 648 \mathrm{D}}$ GOF mutation rendered irregular cell shape and size. This phenotype was also present when $C A S 5$ was disrupted from strains constructed to contain $U P C 2^{\mathrm{G} 648 \mathrm{~S}}$ or $U P C 2^{\mathrm{A} 643 \mathrm{~V}}$ alleles. This may suggest a relationship between the two TFs. When UPC2 is constitutively active, ergosterol biosynthesis genes are upregulated and cellular ergosterol content increases (205), consequently impacting membrane fluidity which affects cell wall composition (245). CAS5 is the putative transcriptional regulator of the cell wall integrity pathway (134), and upon its disruption in the presence of a UPC2 GOF mutation, it is possible the cell cannot effectively regulate the morphology of its cell wall and membrane. Also, the similarity of Cas $5 \mathrm{p}$ and Upc2p-dependent genes (discussed below) suggests cross talk between the two transcription factors. Studies to examine such a relationship may uncover other currently unknown roles for each TF.

The strain containing the $E R G 11^{\mathrm{K} 143 \mathrm{R}}$ mutation had an MIC of $4 \mu \mathrm{g} / \mathrm{ml}$ at 24 hours, and $8 \mu \mathrm{g} / \mathrm{ml}$ at 48 and 72 hours in YPD. Although this background was not as highly resistant as others, its respective $C A S 5$ deletion mutant had a drop in both fluconazole MIC and MFC at 48 and 72 hours. The $M R R 1^{\mathrm{P} 683 \mathrm{~S}}$ and $T A C 1^{\mathrm{G} 980 \mathrm{E}}$ resistant strains both had MICs of $8 \mu \mathrm{g} / \mathrm{ml}$ at 24 hours, $16 \mu \mathrm{g} / \mathrm{ml}$ at 48 hours and $>64 \mu \mathrm{g} / \mathrm{ml}$ at 72 hours, and MFCs of $>64 \mu \mathrm{g} / \mathrm{ml}$ at all time points in YPD medium. These values dropped moderately in the respective cas $5 \Delta / \Delta$ mutants. A halo of reduced confluent growth was observed around the Etest strip for the $M R R 1^{\mathrm{P} 683 \mathrm{~S}}$ and $T A C 1^{\mathrm{G} 980 \mathrm{E}}$ constructed mutants, but not the ERG $11^{\mathrm{K} 143 \mathrm{R}}$ background. Fitness defects have been shown to be associated with the introduction of resistance mutations and may explain the growth of ERG $11^{\mathrm{K} 143 \mathrm{R}}$ when examined by Etest (225). Despite the absence of reduced confluent growth, the cas $\Delta / \Delta$ mutants in each background showed increased susceptibility to fluconazole by Etest and a decreased ability to grow in the presence of fluconazole at $10 \mu \mathrm{g} / \mathrm{ml}$. 
Although the loss of CAS5 in strains containing a single mechanism of resistance resulted in slightly enhanced susceptibility to fluconazole, this modest effect is not practical for clinical applications in overcoming resistance. The differences in susceptibility observed for the mutants constructed in the azole-resistant clinical isolates versus the isogenic strains may be due to the suggestion that clinical isolates have compensatory mutations mitigating any decrease in fitness resulting from such resistance mutations (225).

We then sought to determine if $C A S 5$ might influence fluconazole susceptibility through altered expression of the efflux pump genes $C D R 1, C D R 2$, and $M D R 1$. The expression of $C D R 1$ was not affected in the absence of CAS5, except for slightly in the $T A C 1^{\mathrm{G} 980 \mathrm{E}}$ background. However, $C D R 2$ and $M D R 1$ expression was upregulated in most backgrounds, except for $M R R I^{\mathrm{P} 683 \mathrm{~S}}$ where $M D R 1$ expression was downregulated. The change in susceptibility for the $M R R 1^{\mathrm{P} 683 \mathrm{~S}}$ background may be due to decreased $M D R I$ expression, but $C D R 2$ expression is upregulated in this background. Therefore, the moderate effect on susceptibility observed upon disruption of $C A S 5$ does not appear to be due to altered expression of the efflux pumps.

Another hypothesis for the increased susceptibility to fluconazole of the cas $5 \Delta / \Delta$ mutants is changes in cell wall structure. Previous reports have shown that when the cell wall integrity pathway is disrupted, specifically by disrupting the protein kinase gene $M K C 1$, the cell exhibits increased susceptibility to fluconazole $(105,237)$. In response to fluconazole, the upstream kinase Pkclp phosphorylates Mkclp, but is interrupted by its absence. Cas $5 p$ has been postulated to be the functional equivalent of the Saccharomyces cerevisiae cell wall integrity downstream transcriptional regulator ScRlmlp (134). As previous reports have suggested that cell wall integrity is critical for the fungistatic effect of sterol biosynthesis inhibitors (246), this implicates that when CAS5 is disrupted the integrity of the cell wall is compromised and results in increased susceptibility. Indeed, expression of cellular cell wall integrity genes measured by microarray analysis was reduced in the cas $5 \Delta / \triangle$ mutant as compared to its parent strain SC5314 upon exposure to fluconazole. PGA13, PGA31, RCA1, VPS7, VPS28, orf19.5644, and orf19.719 genes are all involved in cell wall integrity. Interestingly, the gene ontology process categories of iron ion transport and iron ion homeostasis were also found to be dependent upon CAS5. This is consistent with the involvement of cell wall proteins in iron acquisition (247).

Previous studies by our group also identified a similar set of iron ion transport and iron ion homeostasis genes to be dependent upon UPC2 (Chapter 2) (Table 3-8), suggesting crosstalk between the two transcription factors. Indeed upon disruption of $C A S 5$, the baseline expression of $E R G 11$ was reduced in all resistant backgrounds as measured by real-time RT-PCR. Additionally, several genes involved in the lipid metabolic process were also identified to be Cas5-dependent, including $E R G 26$ of the ergosterol biosynthesis pathway, a process that is regulated by $\operatorname{Upc} 2(199,221)$. ERG26 encodes for C-4 sterol decarboxylase and has been found to be an essential gene in $C$. albicans (248). Therefore, inhibition of this step may represent a potential drug target.

These data suggest that the enhanced activity of fluconazole observed in both susceptible and resistant strains lacking $C A S 5$ may be due to deregulation of iron ion 
Table 3-8. Genes upregulated by at least 1.5-fold by fluconazole which are dependent upon Cas5 and Upc2

\begin{tabular}{|c|c|c|c|c|c|c|c|c|c|c|c|c|}
\hline \multirow[b]{3}{*}{ Process $^{a}$} & \multirow{3}{*}{$\begin{array}{c}\text { orf19 } \\
\text { Designation }\end{array}$} & \multirow{3}{*}{$\begin{array}{l}\text { CGD } \\
\text { Name }\end{array}$} & \multicolumn{6}{|c|}{ Change in Fold Expression } & & & & \\
\hline & & & \multicolumn{2}{|c|}{$\begin{array}{c}\text { SC5314+FLC/ } \\
\text { SC53314 (A) } \\
\end{array}$} & \multicolumn{2}{|c|}{$\begin{array}{c}\operatorname{cas} 5 \Delta / \Delta+\mathrm{FLC} / \\
\operatorname{cas} 5 \Delta / \Delta(\mathrm{B}) \\
\end{array}$} & \multicolumn{2}{|c|}{$\begin{array}{c}u p c 2 \Delta / \Delta+F L C / \\
u p c 2 \Delta / \Delta(C)\end{array}$} & \multicolumn{2}{|c|}{$\begin{array}{c}\text { CAS5 Ratio } \\
(\mathrm{B} / \mathrm{A})\end{array}$} & \multicolumn{2}{|c|}{$\begin{array}{c}\text { UPC2 Ratio } \\
\text { C/A) }\end{array}$} \\
\hline & & & Exp1 & Exp2 & Exp1 & Exp2 & Exp1 & Exp2 & Exp1 & Exp2 & Exp1 & Exp2 \\
\hline \multirow{2}{*}{$\begin{array}{l}\text { Lipid metabolic } \\
\text { process }\end{array}$} & orf19.2670 & & 1.6 & 1.8 & 0.4 & 0.4 & 0.4 & 0.7 & 0.3 & 0.2 & 0.2 & 0.4 \\
\hline & orf19.4982 & & 2.1 & 2.3 & 0.9 & 0.6 & 0.7 & 0.8 & 0.4 & 0.3 & 0.3 & 0.4 \\
\hline \multirow[t]{4}{*}{ Iron ion transport } & orf19.1415 & FRE10 & 2.0 & 4.2 & 0.9 & 2.3 & 0.2 & 0.1 & 0.5 & 0.5 & 0.1 & 0.0 \\
\hline & orf19.1932 & CFL4 & 35.6 & 359.0 & 2.4 & 6.6 & 8.0 & 22.0 & 0.1 & 0.0 & 0.2 & 0.1 \\
\hline & orf19.4215 & FET34 & 2.1 & 5.2 & 0.2 & 0.5 & 0.0 & 0.1 & 0.1 & 0.1 & 0.0 & 0.0 \\
\hline & orf19.7219 & FTR1 & 3.2 & 7.8 & 0.5 & 1.1 & 0.1 & 0.0 & 0.1 & 0.1 & 0.0 & 0.0 \\
\hline Iron ion homeostasis & orf19.1264 & CFL2 & 2.0 & 6.0 & 0.6 & 0.5 & 0.2 & 0.8 & 0.3 & 0.1 & 0.1 & 0.1 \\
\hline \multirow[t]{9}{*}{ Transport } & orf19.1352 & TIM22 & 3.6 & 4.4 & 0.9 & 1.1 & 1.3 & 2.0 & 0.2 & 0.3 & 0.4 & 0.4 \\
\hline & orf19.2292 & OPT4 & 5.2 & 3.7 & 1.1 & 1.0 & 0.0 & 0.0 & 0.2 & 0.3 & 0.0 & 0.0 \\
\hline & orf19.2350 & & 2.5 & 4.1 & 0.6 & 1.0 & 0.9 & 0.6 & 0.3 & 0.3 & 0.4 & 0.1 \\
\hline & orf19.3195 & HIP1 & 1.5 & 2.6 & 0.6 & 0.7 & 0.7 & 0.8 & 0.4 & 0.3 & 0.5 & 0.3 \\
\hline & orf19.3232 & & 24.6 & 5.3 & 2.6 & 1.0 & 1.6 & 1.6 & 0.1 & 0.2 & 0.1 & 0.3 \\
\hline & orf19.4384 & HXT5 & 70.6 & 75.5 & 11.1 & 23.4 & 16.9 & 9.7 & 0.2 & 0.3 & 0.2 & 0.1 \\
\hline & orf19.4682 & HGT17 & 45.0 & 24.7 & 7.0 & 7.3 & 4.2 & 4.5 & 0.2 & 0.3 & 0.1 & 0.2 \\
\hline & orf19.6249 & HAK1 & 4.4 & 10.7 & 2.1 & 3.2 & 2.0 & 2.6 & 0.5 & 0.3 & 0.5 & 0.2 \\
\hline & orf19.6993 & $G A P 2$ & 21.7 & 8.6 & 3.0 & 4.5 & 3.6 & 2.5 & 0.1 & 0.5 & 0.2 & 0.3 \\
\hline \multirow[t]{4}{*}{ Response to stress } & orf19.1434 & & 1.7 & 2.7 & 0.8 & 1.0 & 0.8 & 0.9 & 0.5 & 0.4 & 0.5 & 0.3 \\
\hline & orf19.3239 & CTF 18 & 2.7 & 2.4 & 1.0 & 1.1 & 1.4 & 0.8 & 0.4 & 0.5 & 0.5 & 0.4 \\
\hline & orf19.4317 & GRE3 & 1.5 & 1.5 & 0.8 & 0.6 & 0.3 & 0.7 & 0.5 & 0.4 & 0.2 & 0.5 \\
\hline & orf19.5902 & $R A S 2$ & 7.6 & 6.2 & 0.8 & 1.1 & 2.3 & 1.5 & 0.1 & 0.2 & 0.3 & 0.2 \\
\hline \multirow{2}{*}{$\begin{array}{c}\text { Response to } \\
\text { chemical stimulus }\end{array}$} & orf19.5591 & $A D O 1$ & 2.4 & 2.9 & 1.1 & 1.5 & 0.7 & 0.8 & 0.4 & 0.5 & 0.3 & 0.3 \\
\hline & orf19.6102 & $R C A 1$ & 2.5 & 3.4 & 1.2 & 1.2 & 1.1 & 1.4 & 0.5 & 0.4 & 0.5 & 0.4 \\
\hline
\end{tabular}


Table 3-8. (Continued)

\begin{tabular}{|c|c|c|c|c|c|c|c|c|c|c|c|c|}
\hline \multirow[b]{3}{*}{ Process $^{\mathrm{a}}$} & \multirow{3}{*}{$\begin{array}{c}\text { orf19 } \\
\text { Designation }\end{array}$} & \multirow{3}{*}{$\begin{array}{c}\text { CGD } \\
\text { Name }\end{array}$} & \multicolumn{6}{|c|}{ Change in Fold Expression } & \multirow{2}{*}{\multicolumn{2}{|c|}{$\begin{array}{c}\text { CAS5 Ratio } \\
\text { (B/A) }\end{array}$}} & \multirow{2}{*}{\multicolumn{2}{|c|}{$\begin{array}{l}\text { UPC2 Ratio } \\
\text { C/A) }\end{array}$}} \\
\hline & & & \multicolumn{2}{|c|}{$\begin{array}{l}\text { SC5314+FLC/ } \\
\text { SC5314 (A) }\end{array}$} & \multicolumn{2}{|c|}{$\begin{array}{c}\operatorname{cas} 5 \Delta / \Delta+\mathrm{FLC} / \\
\operatorname{cas} 5 \Delta / \Delta(\mathrm{B})\end{array}$} & \multicolumn{2}{|c|}{$\begin{array}{c}u p c 2 \Delta / \Delta+F L C / \\
u p c 2 \Delta / \Delta(C)\end{array}$} & & & & \\
\hline & & & Exp1 & Exp2 & Exp1 & Exp2 & Exp1 & Exp2 & Exp1 & Exp2 & Exp1 & Exp2 \\
\hline \multirow{4}{*}{$\begin{array}{l}\text { Oxidation-reduction } \\
\text { process }\end{array}$} & orf19.7374 & CTA4 & 1.7 & 2.2 & 0.8 & 1.1 & 0.8 & 1.0 & 0.5 & 0.5 & 0.5 & 0.5 \\
\hline & orf19.1411 & & 2.7 & 4.8 & 1.1 & 0.9 & 1.4 & 1.1 & 0.4 & 0.2 & 0.5 & 0.2 \\
\hline & orf19.2108 & SOD6 & 7.9 & 10.6 & 1.6 & 1.1 & 1.1 & 1.6 & 0.2 & 0.1 & 0.1 & 0.1 \\
\hline & orf19.4747 & HEM14 & 1.7 & 2.0 & 0.6 & 0.4 & 0.1 & 0.2 & 0.3 & 0.2 & 0.0 & 0.1 \\
\hline Filamentous growth & orf19.5741 & $A L S 1$ & 66.8 & 79.0 & 22.6 & 21.0 & 4.8 & 3.1 & 0.3 & 0.3 & 0.1 & 0.0 \\
\hline Biofilm formation & orf19.4899 & $G C A 1$ & 4.8 & 4.4 & 0.9 & 1.2 & 0.2 & 0.6 & 0.2 & 0.3 & 0.0 & 0.1 \\
\hline \multirow[t]{3}{*}{ Other } & orf19.410.3 & RIB4 & 5.6 & 5.7 & 0.6 & 0.5 & 1.0 & 0.8 & 0.1 & 0.1 & 0.2 & 0.1 \\
\hline & orf19.467 & WOR3 & 32.3 & 7.1 & 3.0 & 2.9 & 2.8 & 0.8 & 0.1 & 0.4 & 0.1 & 0.1 \\
\hline & orf19.6057 & & 2.5 & 1.8 & 0.9 & 1.0 & 0.2 & 0.2 & 0.3 & 0.5 & 0.1 & 0.1 \\
\hline \multirow[t]{14}{*}{ Unknown } & orf19.1277 & & 6.6 & 9.3 & 1.5 & 1.5 & 3.4 & 2.7 & 0.2 & 0.2 & 0.5 & 0.3 \\
\hline & orf19.2414 & & 3.1 & 3.3 & 1.1 & 0.6 & 0.7 & 1.7 & 0.4 & 0.2 & 0.2 & 0.5 \\
\hline & orf19.2691 & & 1.5 & 1.5 & 0.5 & 0.7 & 0.4 & 0.7 & 0.3 & 0.4 & 0.3 & 0.5 \\
\hline & orf19.2836 & & 11.7 & 21.9 & 3.1 & 4.8 & 1.6 & 1.2 & 0.3 & 0.2 & 0.1 & 0.1 \\
\hline & orf19.2839 & CIRT4B & 1.6 & 2.6 & 0.4 & 0.3 & 0.7 & 0.6 & 0.2 & 0.1 & 0.4 & 0.2 \\
\hline & orf19.3499 & & 79.5 & 20.5 & 5.9 & 7.6 & 10.6 & 10.1 & 0.1 & 0.4 & 0.1 & 0.5 \\
\hline & orf19.3881 & & 1.9 & 2.0 & 0.7 & 0.6 & 0.5 & 0.5 & 0.4 & 0.3 & 0.2 & 0.2 \\
\hline & orf19.4013 & & 2.6 & 2.2 & 0.6 & 0.6 & 0.3 & 0.4 & 0.2 & 0.3 & 0.1 & 0.2 \\
\hline & orf19.4144 & & 2.4 & 3.2 & 1.2 & 1.6 & 0.9 & 0.9 & 0.5 & 0.5 & 0.4 & 0.3 \\
\hline & orf19.4658 & & 5.0 & 6.6 & 2.5 & 3.1 & 2.4 & 1.1 & 0.5 & 0.5 & 0.5 & 0.2 \\
\hline & orf19.5019 & & 2.8 & 3.4 & 1.4 & 1.8 & 0.9 & 1.3 & 0.5 & 0.5 & 0.3 & 0.4 \\
\hline & orf19.6186 & & 12.1 & 13.3 & 2.4 & 2.0 & 2.8 & 2.7 & 0.2 & 0.1 & 0.2 & 0.2 \\
\hline & orf19.6690 & & 2.4 & 2.3 & 0.7 & 0.6 & 0.6 & 0.7 & 0.3 & 0.3 & 0.3 & 0.3 \\
\hline & orf19.670.2 & & 47.4 & 152.7 & 10.8 & 16.1 & 18.3 & 14.1 & 0.2 & 0.1 & 0.4 & 0.1 \\
\hline
\end{tabular}


Table 3-8. (Continued)

\begin{tabular}{|c|c|c|c|c|c|c|c|c|c|c|c|c|}
\hline \multirow[b]{3}{*}{ Process $^{\mathrm{a}}$} & \multirow[b]{3}{*}{$\begin{array}{c}\text { orf19 } \\
\text { Designation } \\
\end{array}$} & \multirow[b]{3}{*}{$\begin{array}{l}\text { CGD } \\
\text { Name } \\
\end{array}$} & \multicolumn{6}{|c|}{$\begin{array}{c}\text { Change in Fold Expression } \\
\end{array}$} & \multirow{2}{*}{\multicolumn{2}{|c|}{$\begin{array}{c}\text { CAS5 Ratio } \\
(\mathrm{B} / \mathrm{A})\end{array}$}} & & \\
\hline & & & \multicolumn{2}{|c|}{$\begin{array}{c}\text { SC5314+FLC/ } \\
\text { SC5314 (A) }\end{array}$} & \multicolumn{2}{|c|}{$\begin{array}{c}\operatorname{cas} 5 \Delta / \Delta+\mathrm{FLC} / \\
\operatorname{cas} 5 \Delta / \Delta(\mathrm{B})\end{array}$} & \multicolumn{2}{|c|}{$\begin{array}{c}u p c 2 \Delta / \Delta+\mathrm{FLC} / \\
\operatorname{upc} 2 \Delta / \Delta(\mathrm{C})\end{array}$} & & & \multicolumn{2}{|c|}{$\begin{array}{c}\text { UPC2 Ratio } \\
\text { C/A) }\end{array}$} \\
\hline & & & Exp1 & Exp2 & Exp1 & Exp2 & Exp1 & Exp2 & Exp1 & Exp2 & Exp1 & Exp2 \\
\hline & orf19.6905 & & 2.4 & 3.3 & 0.5 & 0.4 & 1.2 & 1.4 & 0.2 & 0.1 & 0.5 & 0.4 \\
\hline & orf19.6970 & & 4.5 & 4.4 & 2.3 & 2.4 & 2.0 & 1.8 & 0.5 & 0.5 & 0.4 & 0.4 \\
\hline & orf19.6983 & & 2.1 & 9.1 & 1.0 & 1.3 & 0.4 & 0.0 & 0.5 & 0.1 & 0.2 & 0.0 \\
\hline & orf19.7567 & & 2.5 & 2.4 & 1.1 & 0.8 & 0.2 & 0.5 & 0.4 & 0.3 & 0.1 & 0.2 \\
\hline & orf19.951 & & 2.3 & 2.4 & 0.4 & 0.4 & 0.8 & 1.2 & 0.2 & 0.2 & 0.3 & 0.5 \\
\hline
\end{tabular}

${ }^{a}$ Descriptions are from the Candida Genome Database (http://www.candidagenome.org). 
transport and ERG26 expression, in addition to the inability to upregulate genes involved in the cell wall integrity pathway. Delineation of which downstream targets influence azole susceptibility would identify potential targets for co-therapeutic strategies rendering enhanced activity to the azoles, and as a result may mitigate the development of resistance. 


\section{CHAPTER 4. DISCUSSION}

Candida albicans is the most prevalent human fungal pathogen and causes a wide spectrum of diseases (65). Despite this there are few fundamentally different classes of clinical antifungals available for treatment, due to limited unique and important fungal targets which are not found in the host (61). Currently available antifungal drugs act primarily on the fungal cell wall and ergosterol found in the cell membrane. Perturbation of either of these structures or their components impacts the fungal cell's survival. Fungal cell walls display an electron-dense fibrillar outer layer composed of mannoproteins, and an inner layer of glucans and chitin conferring elasticity and tensile strength to the cell wall $(62,63)$. Ergosterol is found in the fungal cell membrane and regulates fluidity and ultimately membrane integrity (64). Both are exploitable drug targets as the cell wall is essential for survival, and human cells lack both cell walls and ergosterol.

The azole antifungals (such as fluconazole) are the most widely used and important ergosterol biosynthesis inhibitors (EBIs) for the treatment of Candida infections (65). This class of antifungals acts by inhibiting the product of ERG11, a critical cytochrome $\mathrm{P} 450$ enzyme of the ergosterol biosynthesis pathway, known as lanosterol $14 \alpha$-demethylase. Inhibition of this enzyme results in accumulation of sterol intermediates and altered cell membrane structure and function $(79,82)$. Despite their overall success in treatment, the azoles are fungistatic against $C$. albicans and therefore have limited efficacy against this organism, especially for immunocompromised patients, and the development of resistance can occur $(61,85,206)$. The lack of lethality caused by sterol biosynthesis inhibition may be due to adaptive responses which circumvent azole activity and permit fungal survival in the presence of the azoles. Indeed, various pathways have been shown to be critical for the fungistatic activity of azole antifungals.

We hypothesized that the transcriptional regulators of these pathways are required for the response to the azoles in both susceptible and resistant strains. Identification of their downstream targets would be useful as novel targets for the development of cotherapeutic strategies to improve the efficacy of fluconazole treatment. By studying mutants of specific transcription factors we were able to identify those whose disruption results in enhanced susceptibility in both susceptible and resistant strains. Using $C$. albicans Affymetrix microarrays we were able to examine the genome-wide expression profile of these mutants and their background strains for fluconazole-inducible genes whose expressions are TF-dependent.

Liu et al. recently examined the genome-wide expression profile in response to ketoconazole exposure and observed an induction of genes involved in ergosterol biosynthesis (97). Many of these genes exist upstream from Erg11p, the azole target, and respond to ergosterol depletion by facilitating the production of alternate sterols including

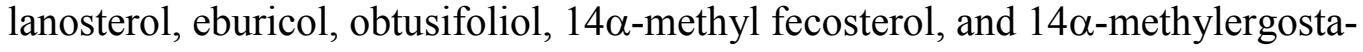
8,24(28)-dien-3 $\beta, 6 \alpha$-diol to be inserted into the cell membrane (107) (Figure 4-1). Integration of these sterols into the plasma membrane disrupts its integrity resulting in its 


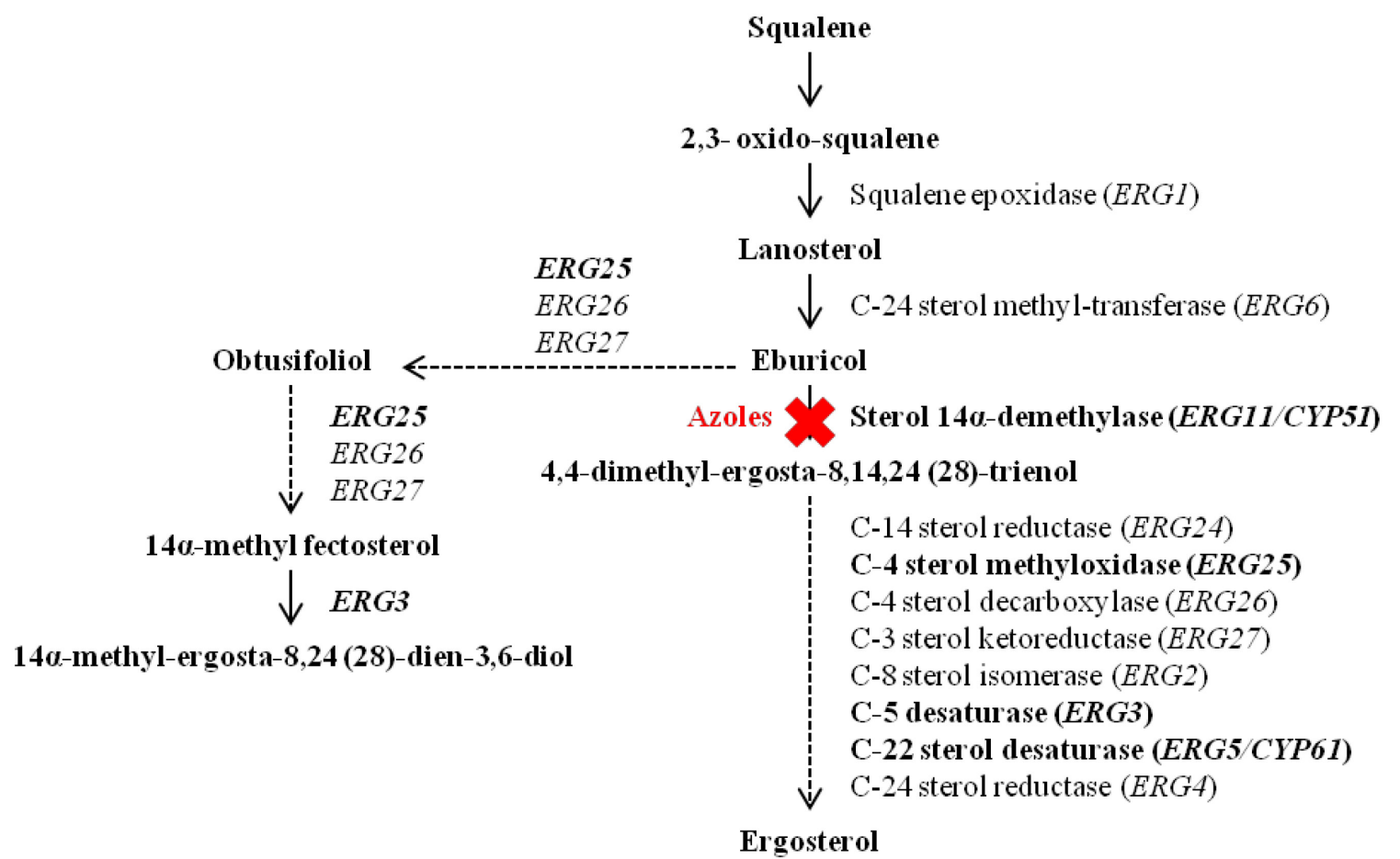

Figure 4-1. An alternate sterol biosynthesis pathway in $C$. albicans

Alternate sterols produced in response to azole inhibition. Genes in boldface have been shown to be influenced by iron. Dotted lines denote multiple enzymatic steps $(232,234$, 235). 
altered structure and function $(79,82,108)$. Under these conditions, growth is inhibited but the organism remains viable (109).

The transcriptional regulator of the ergosterol biosynthesis pathway is Upc2, controlling inducible ergosterol genes expression in response to the azoles. Upon disruption of $U P C 2$, the cells become hypersusceptible to fluconazole, ERG gene inducible transcription is abolished, and as a result ergosterol levels are reduced (199, 200). Our first objective was to better characterize the role of UPC2 in fluconazole susceptibility in both azole-susceptible and azole-resistant strains. In addition to previous observations of reduced fluconazole MIC for a $u p c 2 \Delta / \Delta$ mutant as compared to its azolesusceptible parent strain, we observed a reduced MFC when disrupted from the azolesusceptible parent SC5314 as well as the highly azole-resistant parent 12-99, which carries the four most common mechanisms of resistance. Disruption in both backgrounds prevented its regrowth after 72 hours in the presence of higher fluconazole concentrations in YPD medium, resulted in a clear zone of inhibition around a fluconazole Etest strip, and prevented growth on solid YPD medium containing a therapeutically relevant concentration of fluconazole $(10 \mu \mathrm{g} / \mathrm{ml})$. An enhanced effect for fluconazole was also demonstrated by time-kill analysis for the $u p c 2 \Delta / \Delta$ mutants as compared to their respective parent strains. The same phenotype was also observed upon disruption of $U P C 2$ in isogenic strains containing resistance mutations in ERG11, MRR1, and TAC1. The upc $2 \Delta / \Delta$ mutants in each background showed increased susceptibility to fluconazole by Etest and an inability to grow on solid media containing fluconazole.

C. albicans Upc2 has been shown to bind the promoters of CDR1 and MDR1 (201). However, the observed effect on fluconazole susceptibility occurred without any change in expression of the efflux pump genes $C D R 1, C D R 2$, or $M D R 1$ and therefore is not the cause for how the disruption of UPC2 overcomes drug resistance. UPC2 disruption did result in reduced $E R G 11$ expression compared to both azole-susceptible and azole-resistant parent strains as measured by real-time RT-PCR, and inducible expression of ERG6, ERG24, and ERG27 (as well as ERG11) was reduced in the upc $2 \Delta / \Delta$ mutant as compared to its parent strain SC5314 upon exposure to fluconazole as measured by microarray analysis. Such changes in expression would diminish the intracellular drug target and as well as the biosynthesis of alternative sterols, compromising the organism in the presence of fluconazole.

Interestingly, genes involved in iron transport (CFL4, FET3, FET34, FRE10, $F R P 1, F T R 1)$ and homeostasis (CFL2, CSA1, IRO1, RBT5) were also found to be dependent upon $U P C 2$ for their induction in response to fluconazole. Before being taken up by the cell, ferric iron must be solubilized to its ferrous form by a cell surface ferric reductase ( $F R E 1$, also known as FRE10), and when iron availability is low it is then taken up by the high-affinity iron uptake system including a membrane permease (FTR1) and a multicopper oxidase (FET3) (249-251) (Figure 4-2). Disruption of FTR1 and iron starvation have previously been shown to enhance fluconazole susceptibility in $C$. albicans (228-231) and the iron uptake system has been proposed as an alternative target for combination antifungal treatment (233). Iron deprived cells also have decreased amounts of both ergosterol and sterol intermediates when compared to iron sufficient 


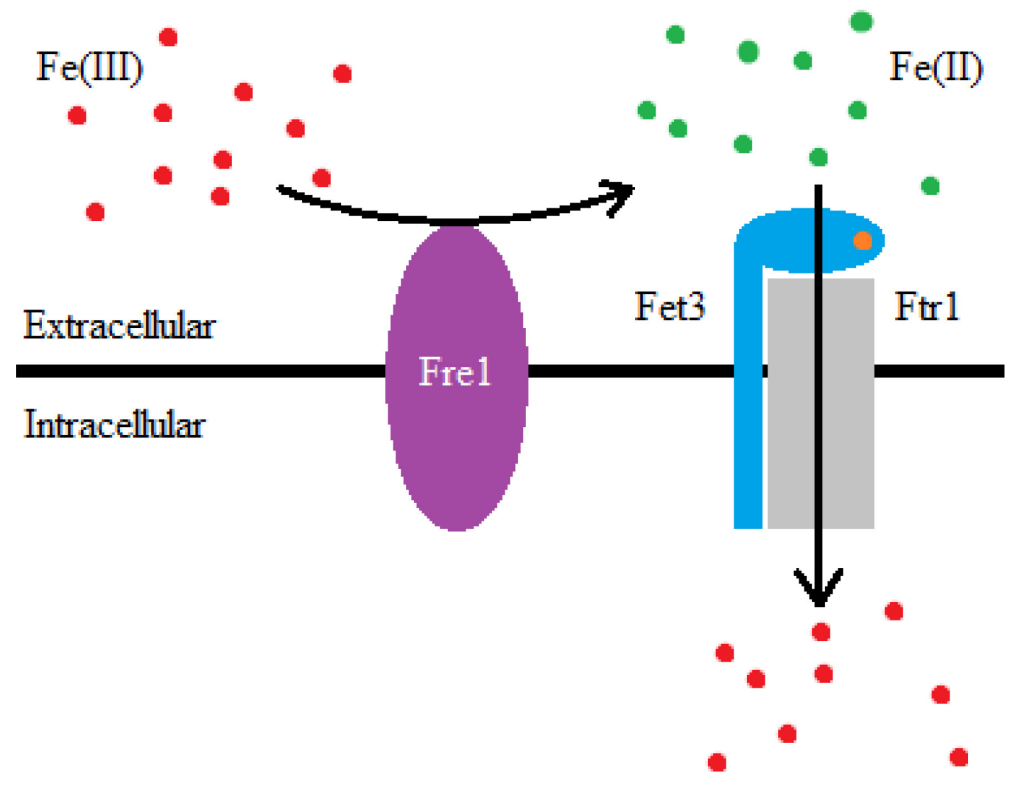

Figure 4-2. High-affinity ion uptake in yeast

Extracellular ferric iron is reduced to ferrous iron by a ferric reductase (Fre1). A multicopper oxidase (Fet3) oxidizes the iron back to its ferric form which is then transported into the cell by an iron permease (Ftr1) (252). 
cells $(228,253)$. This may be due to the inactivity of enzymes in the ergosterol biosynthesis pathways which require iron for their function. ERG11 and ERG5 encode cytochrome P450 enzymes and their active sites contain a heme iron. Also, the expressions ERG11 and ERG3 have been shown to be iron regulated, and while ERG25 expression is not regulated by iron, it is an iron containing gene identified to be essential to viability (253-256). Since many enzymes in the ergosterol biosynthesis pathway require iron, reduced expression of iron transport and homeostasis genes would impact the activity of ergosterol biosynthesis enzymes in response to fluconazole. Therefore, the enhanced effect of fluconazole upon the disruption of UPC2 may be due to this effect in combination with decreased expression of the $E R G$ genes. Indeed, a upc $2 \Delta / \Delta$ mutant has been shown to be highly susceptible to Malachite green, a compound which depletes intracellular iron (257).

We then sought out to identify other transcription factors that had similar effects on fluconazole susceptibility as UPC2 by screening a library of mutants disrupted for genes encoding transcription factors (187). Our results in Chapter 3 showed that the TF Cas 5 was identified to be hypersusceptible to fluconazole, and independent disruption of $C A S 5$ from the azole-susceptible parent SC5314 also exhibited increased susceptibility to fluconazole. The fluconazole MIC was slightly reduced and a substantial reduction was observed for the fluconazole MFC. Disruption of CAS5 prevented regrowth after 72 hours in the presence of higher fluconazole concentrations in YPD medium, resulted in a clear zone of inhibition around a fluconazole Etest strip, and prevented growth on solid YPD medium containing a therapeutically relevant concentration of fluconazole (10 $\mu \mathrm{g} / \mathrm{ml}$ ). An enhanced effect for fluconazole was also demonstrated by time-kill analysis as compared to the parent strain. This phenotype was not as strong when disrupted in isogenic strains containing resistance mutations in ERG11, MRR1, and TAC1 and had no effect on azole-resistant clinical isolates. Of note, the cas $5 \Delta / \Delta$ mutant derived from a strain containing resistance mutations in UPC2 resulted in altered cell shape and size, suggesting a relationship between the two transcription factors, but was unable to be quantified due to cell density irregularities. The cas $5 \Delta / \Delta$ mutants in the ERG11, MRR1, and $T A C 1$ backgrounds showed slightly increased susceptibility to fluconazole by Etest and reduced inability to grow on solid media containing fluconazole. The effect of CAS5 on fluconazole susceptibility was not shown to be due to decreased expression of the $C D R 1, C D R 2$, and MDR 1 efflux pump genes. Although the resulting modest effect on resistance is not practical for clinical application, it is important to note the enhanced effect in the susceptible background which would improve efficacy of treatment. As a result, it may also impact the development of resistance.

The cas $5 \Delta / \Delta$ mutants in the ERG11, MRR1, and TAC1 backgrounds did exhibit a decrease in $E R G 11$ expression, adding evidence to a possible relationship between Cas5p and Upc2, as inducible ERG11 expression is controlled by Upc2. Additionally, inducible expression of several genes involved in the lipid metabolic process (including ERG26) were reduced in the cas $5 \Delta / \Delta$ mutant as compared to its parent strain SC5314 upon exposure to fluconazole as measured by microarray analysis. ERG 26 encodes for C-4 sterol decarboxylase which is an essential enzyme in $C$. albicans for viability (248). As previously mentioned, decreased expression ERG11 would diminish the target of the 
azoles and decreased ERG26 expression would impact the biosynthesis of alternative sterols, compromising the ability of this response mechanism to circumvent the activity of fluconazole. Another striking similarity to $U P C 2$ was the dependence of iron ion transport (CFL4, FET34, FRE10, FTR1), binding (orf19.1411), and homeostasis (CFL2) genes on Cas 5 by microarray analysis. Many of these genes were previously identified to also be dependent upon Upc2 (Chapter 2). The disruption of cell membrane integrity by fluconazole also impacts cell wall integrity (258). Since cell membrane and wall proteins such as Cfl2p, Cfl4p, Fet3p, Fet34p, Fre10p, and Ftr1p are involved in iron acquisition (247), it is reasonable for a relationship to exist in regulating iron acquisition and cell wall maintenance genes.

Cas5 has been postulated to be the transcriptional regulator of the CWI pathway (239), which is required to maintain cell shape and integrity (134). The specificity of the CWI pathway to fungal cells and its role in responding to fluconazole activity makes it an attractive system for which novel co-therapeutic agents could be designed to act synergistically with fluconazole $(103,104)$. When the cas $5 \Delta / \Delta$ mutant was compared to its parent strains by microarray analysis, several genes involved in cell wall organization were identified to dependent upon Cas 5 for their induction in response to fluconazole. Included were the genes PGA13, PGA31, RCA1, VPS28, YPS7, orf19.5644, and orf19.719. Previous studies also identified that the expression of $P G A 13$ was dependent upon Cas5 in response to caspofungin (134). PGA13 encodes a GPI protein which is highly expressed during cell wall regeneration (259), and its deletion results in slightly enhanced susceptibility to fluconazole and caspofungin (260). The deletion of VPS 28 has also been shown to result in hypersusceptibility to both the azole (fluconazole, voriconazole) and echinocandin antifungals (caspofungin, micafungin) (261). Since many of these genes encode proteins contributing to cell wall integrity, it suggests that when $C A S 5$ is disrupted the integrity of the cell wall is compromised. This, combined with decreased iron uptake, which occurs at the cell surface, may account for the observed increase in fluconazole susceptibility.

Ciclopirox olamine was introduced more than 3 decades ago as a topical antifungal agent with a very broad spectrum of activity (262). Although its mechanism of action is poorly understood, the chelation of metal ions and the inhibition of irondependent enzymes have been shown to play a role (263). Upon exposure to ciclopirox olamine, the expression of genes encoding drug efflux pumps $(C D R 1, C D R 2)$, iron permease $(F T R 1)$, iron transporter $(F T H 1)$, and iron reductase $(C F L 1)$ were induced, while the addition of iron(III) chloride reversed this effect. The iron chelator bipyridine also induced a similar pattern of gene expression (262). Additionally, no cases of resistance have been reported to ciclopirox olamine. This may be due to its fungicidal mode of action or an irreversible binding of the drug to intracellular structures, preventing its export by efflux pumps (264). To examine if resistance could be developed in vitro, cells were grown in the presence of ciclopirox olamine for 6 months. Expression of $C D R 1$ and $C D R 2$ increased, but there was no decrease in susceptibility or development of resistance (262). A later study showed similar gene expression changes in response to ciclopirox olamine, but mutants disrupted for $C D R 1$ and $C D R 2$, as well as 
a clinically azole-resistant isolate overexpressing $C D R 1$ and $C D R 2$, had no change in susceptibility to ciclopirox olamine (265).

Further emphasis for targeting the iron uptake system in combination with antifungal treatment was provided by the observation that doxycycline acts synergistically with fluconazole (266). Doxycycline is an antimicrobial with ironchelating activity (267-270). The combination of doxycycline with fluconazole resulted in fungicidal activity, and prevented the development of fluconazole resistance. It was also effective against a highly fluconazole-resistant clinical isolate, FH5, with an isochromosome 5L duplication resulting in 4 copies of ERG11 and 4 copies of constitutively active $T A C 1$ (271). These effects were reversed upon the addition of iron to the growth medium. High levels of doxycycline alone did impact growth of the susceptible strain SC5314, but had not impact on the azole-resistant isolate. Interestingly, growth of an S. cerevisiae strain with constitutive ERG11 overexpression was able to resume in the presence of these two drugs. However, overexpression of ERG11 in C. albicans did not restore growth (266). Since the Erg11 protein binds heme, and azoles interact with the heme iron (272), iron depletion could decrease the activity of both Erg11 and the azoles. Further study is necessary in order to determine the extent of iron's role in fluconazole susceptibility.

By studying transcription factor mutants in both azole-susceptible and azoleresistant backgrounds, we were able to identify the involvement of high-affinity transport pathway in the response to azoles, as well as expanding upon pathways previously identified. We successfully identified $U P C 2$ and $C A S 5$ in the regulation of iron ion transport, binding, and homeostasis in addition to the role of Upc2 as the regulator of ergosterol biosynthesis and Cas 5 of cell wall integrity in the presence of fluconazole. Disruption of the transcription factor, Upc2p, was also shown to overcome high levels of azole resistance. Our results demonstrate the importance of using TF mutants for identifying transcriptional pathways functioning in both susceptible and resistant response mechanisms to fluconazole.

The discovery of the role of iron transport and homeostasis may allow for the development of more efficient combination therapy with fluconazole. The combination of iron deprivation and cell wall disruption may be important for preventing the development of azole resistance from the time of therapy initiation. Additionally, targeting both high-affinity iron transport and ergosterol biosynthesis may be able to overcome the existence of resistance as well as hinder its development. 


\section{LIST OF REFERENCES}

1. Ghannoum MA, Jurevic RJ, Mukherjee PK, Cui F, Sikaroodi M, Naqvi A, Gillevet PM. 2010. Characterization of the oral fungal microbiome (mycobiome) in healthy individuals. PLoS Pathog 6:e1000713.

2. Naglik JR, Challacombe SJ, Hube B. 2003. Candida albicans secreted aspartyl proteinases in virulence and pathogenesis. Microbiol Mol Biol Rev 67:400-428, table of contents.

3. Odds FC. 1988. Candida and candidosis: A Review and Bibliography, 2nd ed. Bailliere Tindall, London.

4. Kingsbury JM, Goldstein AL, McCusker JH. 2006. Role of nitrogen and carbon transport, regulation, and metabolism genes for Saccharomyces cerevisiae survival in vivo. Eukaryot Cell 5:816-824.

5. Berman J. 2006. Morphogenesis and cell cycle progression in Candida albicans. Curr Opin Microbiol 9:595-601.

6. Sudbery P, Gow N, Berman J. 2004. The distinct morphogenic states of Candida albicans. Trends Microbiol 12:317-324.

7. Inglis DO, Johnson AD. 2002. Ash1 protein, an asymmetrically localized transcriptional regulator, controls filamentous growth and virulence of Candida albicans. Mol Cell Biol 22:8669-8680.

8. Cottier F, Muhlschlegel FA. 2009. Sensing the environment: response of Candida albicans to the X factor. FEMS Microbiol Lett 295:1-9.

9. Shapiro RS, Uppuluri P, Zaas AK, Collins C, Senn H, Perfect JR, Heitman J, Cowen LE. 2009. Hsp90 orchestrates temperature-dependent Candida albicans morphogenesis via Ras1-PKA signaling. Curr Biol 19:621-629.

10. De Bernardis F, Muhlschlegel FA, Cassone A, Fonzi WA. 1998. The $\mathrm{pH}$ of the host niche controls gene expression in and virulence of Candida albicans. Infect Immun 66:3317-3325.

11. Rogers TJ, Balish E. 1980. Immunity to Candida albicans. Microbiol Rev 44:660-682.

12. Berman J, Sudbery PE. 2002. Candida Albicans: a molecular revolution built on lessons from budding yeast. Nature reviews. Genetics 3:918-930.

13. Baillie GS, Douglas LJ. 1999. Role of dimorphism in the development of Candida albicans biofilms. Journal of medical microbiology 48:671-679.

14. Samaranayake YH, Ye J, Yau JY, Cheung BP, Samaranayake LP. 2005. In vitro method to study antifungal perfusion in Candida biofilms. J Clin Microbiol 43:818-825.

15. Mateus C, Crow SA, Jr., Ahearn DG. 2004. Adherence of Candida albicans to silicone induces immediate enhanced tolerance to fluconazole. Antimicrob Agents Chemother 48:3358-3366.

16. Mukherjee PK, Chandra J, Kuhn DM, Ghannoum MA. 2003. Mechanism of fluconazole resistance in Candida albicans biofilms: phase-specific role of efflux pumps and membrane sterols. Infect Immun 71:4333-4340.

17. Brooun A, Liu S, Lewis K. 2000. A dose-response study of antibiotic resistance in Pseudomonas aeruginosa biofilms. Antimicrob Agents Chemother 44:640-646. 
18. Keren I, Kaldalu N, Spoering A, Wang Y, Lewis K. 2004. Persister cells and tolerance to antimicrobials. FEMS Microbiol Lett 230:13-18.

19. Netea MG, Brown GD, Kullberg BJ, Gow NA. 2008. An integrated model of the recognition of Candida albicans by the innate immune system. Nat Rev Microbiol 6:67-78.

20. Gow NA, Netea MG, Munro CA, Ferwerda G, Bates S, Mora-Montes HM, Walker L, Jansen T, Jacobs L, Tsoni V, Brown GD, Odds FC, Van der Meer JW, Brown AJ, Kullberg BJ. 2007. Immune recognition of Candida albicans beta-glucan by dectin-1. J Infect Dis 196:1565-1571.

21. Wheeler RT, Fink GR. 2006. A drug-sensitive genetic network masks fungi from the immune system. PLoS Pathog 2:e35.

22. Wheeler RT, Kombe D, Agarwala SD, Fink GR. 2008. Dynamic, morphotypespecific Candida albicans beta-glucan exposure during infection and drug treatment. PLoS Pathog 4:e1000227.

23. Mora-Montes HM, Bates S, Netea MG, Castillo L, Brand A, Buurman ET, Diaz-Jimenez DF, Jan Kullberg B, Brown AJ, Odds FC, Gow NA. 2010. A multifunctional mannosyltransferase family in Candida albicans determines cell wall mannan structure and host-fungus interactions. J Biol Chem 285:1208712095.

24. Klippel N, Cui S, Groebe L, Bilitewski U. 2010. Deletion of the Candida albicans histidine kinase gene CHK1 improves recognition by phagocytes through an increased exposure of cell wall beta-1,3-glucans. Microbiology 156:34323444.

25. Galan-Diez M, Arana DM, Serrano-Gomez D, Kremer L, Casasnovas JM, Ortega M, Cuesta-Dominguez A, Corbi AL, Pla J, Fernandez-Ruiz E. 2010. Candida albicans beta-glucan exposure is controlled by the fungal CEK1mediated mitogen-activated protein kinase pathway that modulates immune responses triggered through dectin-1. Infect Immun 78:1426-1436.

26. Perez-Garcia LA, Diaz-Jimenez DF, Lopez-Esparza A, Mora-Montes HM. 2012. Role of Cell Wall Polysaccharides during Recognition of Candida albicans by the Innate Immune System. J Glycobiology 1:102.

27. Braun BR, van Het Hoog M, d'Enfert C, Martchenko M, Dungan J, Kuo A, Inglis DO, Uhl MA, Hogues H, Berriman M, Lorenz M, Levitin A, Oberholzer U, Bachewich C, Harcus D, Marcil A, Dignard D, Iouk T, Zito R, Frangeul L, Tekaia F, Rutherford K, Wang E, Munro CA, Bates S, Gow NA, Hoyer LL, Kohler G, Morschhauser J, Newport G, Znaidi S, Raymond M, Turcotte B, Sherlock G, Costanzo M, Ihmels J, Berman J, Sanglard D, Agabian N, Mitchell AP, Johnson AD, Whiteway M, Nantel A. 2005. A human-curated annotation of the Candida albicans genome. PLoS genetics 1:3657.

28. Hull CM, Raisner RM, Johnson AD. 2000. Evidence for mating of the "asexual" yeast Candida albicans in a mammalian host. Science 289:307-310.

29. Kakar SN, Partridge RM, Magee PT. 1983. A genetic analysis of Candida albicans: isolation of a wide variety of auxotrophs and demonstration of linkage and complementation. Genetics 104:241-255. 
30. Whelan WL, Partridge RM, Magee PT. 1980. Heterozygosity and segregation in Candida albicans. Molecular \& general genetics : MGG 180:107-113.

31. Magee PT, Gale C, Berman J, Davis D. 2003. Molecular genetic and genomic approaches to the study of medically important fungi. Infect Immun 71:22992309.

32. Morschhauser J, Michel S, Staib P. 1999. Sequential gene disruption in Candida albicans by FLP-mediated site-specific recombination. Mol Microbiol 32:547-556.

33. Wirsching S, Michel S, Morschhauser J. 2000. Targeted gene disruption in Candida albicans wild-type strains: the role of the MDR1 gene in fluconazole resistance of clinical Candida albicans isolates. Mol Microbiol 36:856-865.

34. Reuss O, Vik A, Kolter R, Morschhauser J. 2004. The SAT1 flipper, an optimized tool for gene disruption in Candida albicans. Gene 341:119-127.

35. Horn DL, Neofytos D, Anaissie EJ, Fishman JA, Steinbach WJ, Olyaei AJ, Marr KA, Pfaller MA, Chang CH, Webster KM. 2009. Epidemiology and outcomes of candidemia in 2019 patients: data from the prospective antifungal therapy alliance registry. Clin Infect Dis 48:1695-1703.

36. Kollef MH, Napolitano LM, Solomkin JS, Wunderink RG, Bae IG, Fowler VG, Balk RA, Stevens DL, Rahal JJ, Shorr AF, Linden PK, Micek ST. 2008. Health care-associated infection (HAI): a critical appraisal of the emerging threatproceedings of the HAI Summit. Clin Infect Dis 47 Suppl 2:S55-99; quiz S100101.

37. Neofytos D, Horn D, Anaissie E, Steinbach W, Olyaei A, Fishman J, Pfaller M, Chang C, Webster K, Marr K. 2009. Epidemiology and outcome of invasive fungal infection in adult hematopoietic stem cell transplant recipients: analysis of Multicenter Prospective Antifungal Therapy (PATH) Alliance registry. Clin Infect Dis 48:265-273.

38. Perlroth J, Choi B, Spellberg B. 2007. Nosocomial fungal infections: epidemiology, diagnosis, and treatment. Med Mycol 45:321-346.

39. Richardson M, Lass-Florl C. 2008. Changing epidemiology of systemic fungal infections. Clin Microbiol Infect 14 Suppl 4:5-24.

40. Zaoutis TE, Heydon K, Localio R, Walsh TJ, Feudtner C. 2007. Outcomes attributable to neonatal candidiasis. Clin Infect Dis 44:1187-1193.

41. Fishman JA. 2007. Infection in solid-organ transplant recipients. N Engl J Med 357:2601-2614.

42. Mean M, Marchetti O, Calandra T. 2008. Bench-to-bedside review: Candida infections in the intensive care unit. Crit Care 12:204.

43. Procop GW, Roberts GD. 2004. Emerging fungal diseases: the importance of the host. Clin Lab Med 24:691-719, vi-vii.

44. Zaoutis TE, Argon J, Chu J, Berlin JA, Walsh TJ, Feudtner C. 2005. The epidemiology and attributable outcomes of candidemia in adults and children hospitalized in the United States: a propensity analysis. Clin Infect Dis 41:12321239. 
45. Wisplinghoff H, Bischoff T, Tallent SM, Seifert H, Wenzel RP, Edmond MB. 2004. Nosocomial bloodstream infections in US hospitals: analysis of 24,179 cases from a prospective nationwide surveillance study. Clin Infect Dis 39:309317.

46. Pittet D, Li N, Woolson RF, Wenzel RP. 1997. Microbiological factors influencing the outcome of nosocomial bloodstream infections: a 6-year validated, population-based model. Clin Infect Dis 24:1068-1078.

47. Pfaller MA, Diekema DJ. 2007. Epidemiology of invasive candidiasis: a persistent public health problem. Clin Microbiol Rev 20:133-163.

48. Wenzel RP, Gennings C. 2005. Bloodstream infections due to Candida species in the intensive care unit: identifying especially high-risk patients to determine prevention strategies. Clin Infect Dis 41 Suppl 6:S389-393.

49. Fidel PL, Jr., Sobel JD. 1996. Immunopathogenesis of recurrent vulvovaginal candidiasis. Clin Microbiol Rev 9:335-348.

50. Wilkieson C, Samaranayake LP, MacFarlane TW, Lamey PJ, MacKenzie D. 1991. Oral candidosis in the elderly in long term hospital care. Journal of oral pathology \& medicine : official publication of the International Association of Oral Pathologists and the American Academy of Oral Pathology 20:13-16.

51. Samaranayake LP, Wilkieson CA, Lamey PJ, MacFarlane TW. 1995. Oral disease in the elderly in long-term hospital care. Oral diseases 1:147-151.

52. Feigal DW, Katz MH, Greenspan D, Westenhouse J, Winkelstein W, Jr., Lang W, Samuel M, Buchbinder SP, Hessol NA, Lifson AR, et al. 1991. The prevalence of oral lesions in HIV-infected homosexual and bisexual men: three San Francisco epidemiological cohorts. AIDS 5:519-525.

53. Klein RS, Harris CA, Small CB, Moll B, Lesser M, Friedland GH. 1984. Oral candidiasis in high-risk patients as the initial manifestation of the acquired immunodeficiency syndrome. N Engl J Med 311:354-358.

54. Holmberg K, Meyer RD. 1986. Fungal infections in patients with AIDS and AIDS-related complex. Scand J Infect Dis 18:179-192.

55. Thompson GR, 3rd, Patel PK, Kirkpatrick WR, Westbrook SD, Berg D, Erlandsen J, Redding SW, Patterson TF. 2010. Oropharyngeal candidiasis in the era of antiretroviral therapy. Oral Surg Oral Med Oral Pathol Oral Radiol Endod 109:488-495.

56. de Repentigny L, Lewandowski D, Jolicoeur P. 2004. Immunopathogenesis of oropharyngeal candidiasis in human immunodeficiency virus infection. Clin Microbiol Rev 17:729-759.

57. Glocker E, Grimbacher B. 2010. Chronic mucocutaneous candidiasis and congenital susceptibility to Candida. Current opinion in allergy and clinical immunology 10:542-550.

58. Hernandez-Santos N, Gaffen SL. 2012. Th17 cells in immunity to Candida albicans. Cell host \& microbe 11:425-435.

59. Conti HR, Gaffen SL. 2010. Host responses to Candida albicans: Th17 cells and mucosal candidiasis. Microbes and infection / Institut Pasteur 12:518-527. 
60. Delgado AC, de Jesus Pedro R, Aoki FH, Resende MR, Trabasso P, Colombo AL, de Oliveira MS, Mikami Y, Moretti ML. 2009. Clinical and microbiological assessment of patients with a long-term diagnosis of human immunodeficiency virus infection and Candida oral colonization. Clin Microbiol Infect 15:364-371.

61. Anderson JB. 2005. Evolution of antifungal-drug resistance: mechanisms and pathogen fitness. Nat Rev Microbiol 3:547-556.

62. Osmond BC, Specht CA, Robbins PW. 1999. Chitin synthase III: synthetic lethal mutants and "stress related" chitin synthesis that bypasses the CSD3/CHS6 localization pathway. Proc Natl Acad Sci U S A 96:11206-11210.

63. Lesage G, Bussey H. 2006. Cell wall assembly in Saccharomyces cerevisiae. Microbiol Mol Biol Rev 70:317-343.

64. Parks LW, Casey WM. 1995. Physiological implications of sterol biosynthesis in yeast. Annual review of microbiology 49:95-116.

65. Marie C, White TC. 2009. Genetic Basis of Antifungal Drug Resistance. Curr Fungal Infect Rep 3:163-169.

66. Kato M, Wickner W. 2001. Ergosterol is required for the Sec18/ATP-dependent priming step of homotypic vacuole fusion. EMBO J 20:4035-4040.

67. Warnock DW. 1991. Amphotericin B: an introduction. J Antimicrob Chemother 28 Suppl B:27-38.

68. Heese-Peck A, Pichler H, Zanolari B, Watanabe R, Daum G, Riezman H. 2002. Multiple functions of sterols in yeast endocytosis. Mol Biol Cell 13:26642680.

69. Jin H, McCaffery JM, Grote E. 2008. Ergosterol promotes pheromone signaling and plasma membrane fusion in mating yeast. J Cell Biol 180:813-826.

70. Klose C, Ejsing CS, Garcia-Saez AJ, Kaiser HJ, Sampaio JL, Surma MA, Shevchenko A, Schwille P, Simons K. 2010. Yeast lipids can phase-separate into micrometer-scale membrane domains. J Biol Chem 285:30224-30232.

71. Zhang YQ, Gamarra S, Garcia-Effron G, Park S, Perlin DS, Rao R. 2010. Requirement for ergosterol in V-ATPase function underlies antifungal activity of azole drugs. PLoS Pathog 6:e1000939.

72. Gray KC, Palacios DS, Dailey I, Endo MM, Uno BE, Wilcock BC, Burke MD. 2011. Amphotericin primarily kills yeast by simply binding ergosterol. PNAS 109:2234-2239.

73. Gallis HA, Drew RH, Pickard WW. 1990. Amphotericin B: 30 years of clinical experience. Rev Infect Dis 12:308-329.

74. Epstein JB. 1990. Antifungal therapy in oropharyngeal mycotic infections. Oral Surg Oral Med Oral Pathol 69:32-41.

75. Payne NI, Cosgrove RF, Green AP, Liu L. 1987. In-vivo studies of amphotericin B liposomes derived from proliposomes: effect of formulation on toxicity and tissue disposition of the drug in mice. J Pharm Pharmacol 39:24-28.

76. Juliano RL, Grant CW, Barber KR, Kalp MA. 1987. Mechanism of the selective toxicity of amphotericin B incorporated into liposomes. Mol Pharmacol 31:1-11. 
77. White TC, Marr KA, Bowden RA. 1998. Clinical, cellular, and molecular factors that contribute to antifungal drug resistance. Clin Microbiol Rev 11:382402.

78. Conly J, Rennie R, Johnson J, Farah S, Hellman L. 1992. Disseminated candidiasis due to amphotericin B-resistant Candida albicans. J Infect Dis 165:761-764.

79. Ghannoum MA, Rice LB. 1999. Antifungal agents: mode of action, mechanisms of resistance, and correlation of these mechanisms with bacterial resistance. Clin Microbiol Rev 12:501-517.

80. Brammer KW, Farrow PR, Faulkner JK. 1990. Pharmacokinetics and tissue penetration of fluconazole in humans. Rev Infect Dis 12 Suppl 3:S318-326.

81. Sanati H, Belanger P, Fratti R, Ghannoum M. 1997. A new triazole, voriconazole (UK-109,496), blocks sterol biosynthesis in Candida albicans and Candida krusei. Antimicrob Agents Chemother 41:2492-2496.

82. Klepser ME. 2006. Candida resistance and its clinical relevance. Pharmacotherapy 26:68S-75S.

83. Hay RJ. 1990. Overview of studies of fluconazole in oropharyngeal candidiasis. Rev Infect Dis 12 Suppl 3:S334-337.

84. Washton H. 1989. Review of fluconazole: a new triazole antifungal agent. Diagn Microbiol Infect Dis 12:229S-233S.

85. Levin DE. 2005. Cell wall integrity signaling in Saccharomyces cerevisiae. Microbiol Mol Biol Rev 69:262-291.

86. Johnson EM, Warnock DW, Luker J, Porter SR, Scully C. 1995. Emergence of azole drug resistance in Candida species from HIV-infected patients receiving prolonged fluconazole therapy for oral candidosis. J Antimicrob Chemother 35:103-114.

87. Evans TG, Mayer J, Cohen S, Classen D, Carroll K. 1991. Fluconazole failure in the treatment of invasive mycoses. J Infect Dis 164:1232-1235.

88. Smith D, Boag F, Midgley J, Gazzard B. 1991. Fluconazole resistant candida in AIDS. J Infect 23:345-346.

89. Ruhnke M, Eigler A, Tennagen I, Geiseler B, Engelmann E, Trautmann M. 1994. Emergence of fluconazole-resistant strains of Candida albicans in patients with recurrent oropharyngeal candidosis and human immunodeficiency virus infection. J Clin Microbiol 32:2092-2098.

90. Sanglard D. 2003. Resistance and tolerance mechanisms to antifungal drugs in fungal pathogens. Mycologist 17:74-78.

91. Peterson LR, Shanholtzer CJ. 1992. Tests for bactericidal effects of antimicrobial agents: technical performance and clinical relevance. Clin Microbiol Rev 5:420-432.

92. CLSI. 1998. Methodology for the serum bactericidal test. Approved guideline M21-A.

93. CLSI. 2002. Methods for determining bactericidal activity of antimicrobial agents. Approved standard M25-A.

94. Cowen LE. 2008. The evolution of fungal drug resistance: modulating the trajectory from genotype to phenotype. Nat Rev Microbiol 6:187-198. 
95. Stevens DA, White TC, Perlin DS, Selitrennikoff CP. 2005. Studies of the paradoxical effect of caspofungin at high drug concentrations. Diagn Microbiol Infect Dis 51:173-178.

96. Walker LA, Munro CA, de Bruijn I, Lenardon MD, McKinnon A, Gow NA. 2008. Stimulation of chitin synthesis rescues Candida albicans from echinocandins. PLoS Pathog 4:e1000040.

97. Liu TT, Lee RE, Barker KS, Wei L, Homayouni R, Rogers PD. 2005. Genome-wide expression profiling of the response to azole, polyene, echinocandin, and pyrimidine antifungal agents in Candida albicans. Antimicrob Agents Chemother 49:2226-2236.

98. Jain P, Akula I, Edlind T. 2003. Cyclic AMP signaling pathway modulates susceptibility of candida species and Saccharomyces cerevisiae to antifungal azoles and other sterol biosynthesis inhibitors. Antimicrob Agents Chemother 47:3195-3201.

99. Cruz MC, Goldstein AL, Blankenship JR, Del Poeta M, Davis D, Cardenas ME, Perfect JR, McCusker JH, Heitman J. 2002. Calcineurin is essential for survival during membrane stress in Candida albicans. EMBO J 21:546-559.

100. Onyewu C, Wormley FL, Jr., Perfect JR, Heitman J. 2004. The calcineurin target, Crz1, functions in azole tolerance but is not required for virulence of Candida albicans. Infect Immun 72:7330-7333.

101. Sanglard D, Ischer F, Marchetti O, Entenza J, Bille J. 2003. Calcineurin A of Candida albicans: involvement in antifungal tolerance, cell morphogenesis and virulence. Mol Microbiol 48:959-976.

102. Chauhan N, Kruppa M, Calderone R. 2007. The Ssk1p response regulator and Chk1p histidine kinase mutants of Candida albicans are hypersensitive to fluconazole and voriconazole. Antimicrob Agents Chemother 51:3747-3751.

103. Navarro-Garcia F, Alonso-Monge R, Rico H, Pla J, Sentandreu R, Nombela C. 1998. A role for the MAP kinase gene MKC1 in cell wall construction and morphological transitions in Candida albicans. Microbiology 144 ( Pt 2):411-424.

104. Edlind TD. 2001. 41st ICAAC, Chicago, IL.

105. Agarwal AK, Rogers PD, Baerson SR, Jacob MR, Barker KS, Cleary JD, Walker LA, Nagle DG, Clark AM. 2003. Genome-wide expression profiling of the response to polyene, pyrimidine, azole, and echinocandin antifungal agents in Saccharomyces cerevisiae. J Biol Chem 278:34998-35015.

106. LaFayette SL, Collins C, Zaas AK, Schell WA, Betancourt-Quiroz M, Gunatilaka AA, Perfect JR, Cowen LE. 2010. PKC signaling regulates drug resistance of the fungal pathogen Candida albicans via circuitry comprised of Mkc1, calcineurin, and Hsp90. PLoS Pathog 6:e1001069.

107. Kelly SL, Lamb DC, Corran AJ, Baldwin BC, Kelly DE. 1995. Mode of Action and Resistance to Azole Antifungals Associated with the Formation of 14Alpha-Methylergosta-8,24(28)-Dien-3-Beta,6-Alpha-Diol. Biochemical and Biophysical Research Communications 207:910-915.

108. Kelly SL, Lamb DC, Kelly DE, Manning NJ, Loeffler J, Hebart H, Schumacher U, Einsele H. 1997. Resistance to fluconazole and cross-resistance to amphotericin B in Candida albicans from AIDS patients caused by defective sterol delta5,6-desaturation. FEBS Lett 400:80-82. 
109. Watson PF, Rose ME, Ellis SW, England H, Kelly SL. 1989. Defective sterol C5-6 desaturation and azole resistance: a new hypothesis for the mode of action of azole antifungals. Biochem Biophys Res Commun 164:1170-1175.

110. Taipale M, Jarosz DF, Lindquist S. 2010. HSP90 at the hub of protein homeostasis: emerging mechanistic insights. Nature reviews. Molecular cell biology 11:515-528.

111. Pearl LH, Prodromou C. 2006. Structure and mechanism of the Hsp90 molecular chaperone machinery. Annual review of biochemistry 75:271-294.

112. Cowen LE, Lindquist S. 2005. Hsp90 potentiates the rapid evolution of new traits: drug resistance in diverse fungi. Science 309:2185-2189.

113. Shapiro RS, Zaas AK, Betancourt-Quiroz M, Perfect JR, Cowen LE. 2012. The Hsp90 co-chaperone Sgt1 governs Candida albicans morphogenesis and drug resistance. PloS one 7:e44734.

114. Cowen LE, Singh SD, Kohler JR, Collins C, Zaas AK, Schell WA, Aziz H, Mylonakis E, Perfect JR, Whitesell L, Lindquist S. 2009. Harnessing Hsp90 function as a powerful, broadly effective therapeutic strategy for fungal infectious disease. Proc Natl Acad Sci U S A 106:2818-2823.

115. Fang HM, Wang Y. 2006. RA domain-mediated interaction of Cdc35 with Ras 1 is essential for increasing cellular cAMP level for Candida albicans hyphal development. Mol Microbiol 61:484-496.

116. Klengel T, Liang WJ, Chaloupka J, Ruoff C, Schroppel K, Naglik JR, Eckert SE, Mogensen EG, Haynes K, Tuite MF, Levin LR, Buck J, Muhlschlegel FA. 2005. Fungal adenylyl cyclase integrates CO2 sensing with cAMP signaling and virulence. Curr Biol 15:2021-2026.

117. Bockmuhl DP, Krishnamurthy S, Gerads M, Sonneborn A, Ernst JF. 2001. Distinct and redundant roles of the two protein kinase A isoforms Tpk1p and Tpk2p in morphogenesis and growth of Candida albicans. Mol Microbiol 42:1243-1257.

118. Stoldt VR, Sonneborn A, Leuker CE, Ernst JF. 1997. Efg1p, an essential regulator of morphogenesis of the human pathogen Candida albicans, is a member of a conserved class of bHLH proteins regulating morphogenetic processes in fungi. EMBO J 16:1982-1991.

119. Aramburu J, Heitman J, Crabtree GR. 2004. Calcineurin: a central controller of signalling in eukaryotes. EMBO reports 5:343-348.

120. Imai J, Yahara I. 2000. Role of HSP90 in salt stress tolerance via stabilization and regulation of calcineurin. Mol Cell Biol 20:9262-9270.

121. Cowen LE, Carpenter AE, Matangkasombut O, Fink GR, Lindquist S. 2006. Genetic architecture of Hsp90-dependent drug resistance. Eukaryot Cell 5:21842188.

122. Karababa M, Valentino E, Pardini G, Coste AT, Bille J, Sanglard D. 2006. CRZ1, a target of the calcineurin pathway in Candida albicans. Mol Microbiol 59:1429-1451.

123. Stathopoulos AM, Cyert MS. 1997. Calcineurin acts through the CRZ1/TCN1encoded transcription factor to regulate gene expression in yeast. Genes \& development 11:3432-3444. 
124. Steinbach WJ, Reedy JL, Cramer RA, Jr., Perfect JR, Heitman J. 2007. Harnessing calcineurin as a novel anti-infective agent against invasive fungal infections. Nat Rev Microbiol 5:418-430.

125. Stie J, Fox D. 2008. Calcineurin regulation in fungi and beyond. Eukaryot Cell 7:177-186.

126. Uppuluri P, Nett J, Heitman J, Andes D. 2008. Synergistic effect of calcineurin inhibitors and fluconazole against Candida albicans biofilms. Antimicrob Agents Chemother 52:1127-1132.

127. Alonso-Monge R, Navarro-Garcia F, Molero G, Diez-Orejas R, Gustin M, Pla J, Sanchez M, Nombela C. 1999. Role of the mitogen-activated protein kinase Hog $1 p$ in morphogenesis and virulence of Candida albicans. Journal of bacteriology 181:3058-3068.

128. Alonso-Monge R, Navarro-Garcia F, Roman E, Negredo AI, Eisman B, Nombela C, Pla J. 2003. The Hog 1 mitogen-activated protein kinase is essential in the oxidative stress response and chlamydospore formation in Candida albicans. Eukaryot Cell 2:351-361.

129. Arana DM, Nombela C, Alonso-Monge R, Pla J. 2005. The Pbs2 MAP kinase kinase is essential for the oxidative-stress response in the fungal pathogen Candida albicans. Microbiology 151:1033-1049.

130. Maeda T, Wurgler-Murphy SM, Saito H. 1994. A two-component system that regulates an osmosensing MAP kinase cascade in yeast. Nature 369:242-245.

131. Calera JA, Calderone R. 1999. Histidine kinase, two-component signal transduction proteins of Candida albicans and the pathogenesis of candidosis. Mycoses 42 Suppl 2:49-53.

132. Kruppa M, Goins T, Cutler JE, Lowman D, Williams D, Chauhan N, Menon V, Singh P, Li D, Calderone R. 2003. The role of the Candida albicans histidine kinase [CHK1) gene in the regulation of cell wall mannan and glucan biosynthesis. FEMS yeast research 3:289-299.

133. Kruppa M, Jabra-Rizk MA, Meiller TF, Calderone R. 2004. The histidine kinases of Candida albicans: regulation of cell wall mannan biosynthesis. FEMS yeast research 4:409-416.

134. Bruno VM, Kalachikov S, Subaran R, Nobile CJ, Kyratsous C, Mitchell AP. 2006. Control of the C. albicans cell wall damage response by transcriptional regulator Cas5. PLoS Pathog 2:e21.

135. Cutler JE. 1991. Putative virulence factors of Candida albicans. Annual review of microbiology 45:187-218.

136. Miyasaki SH, Hicks JB, Greenspan D, Polacheck I, MacPhail LA, White TC, Agabian N, Greenspan JS. 1992. The identification and tracking of Candida albicans isolates from oral lesions in HIV-seropositive individuals. Journal of acquired immune deficiency syndromes 5:1039-1046.

137. Schmid J, Tay YP, Wan L, Carr M, Parr D, McKinney W. 1995. Evidence for nosocomial transmission of Candida albicans obtained by $\mathrm{Ca} 3$ fingerprinting. $\mathbf{J}$ Clin Microbiol 33:1223-1230.

138. CLSI. 2012. Reference Method for Broth Dilution Antifungal Susceptibility Testing of Yeasts; Fourth Informational Supplement. CLSI document M27-S4. 
139. Rex JH, Pfaller MA, Galgiani JN, Bartlett MS, Espinel-Ingroff A, Ghannoum MA, Lancaster M, Odds FC, Rinaldi MG, Walsh TJ, Barry AL. 1997. Development of interpretive breakpoints for antifungal susceptibility testing: conceptual framework and analysis of in vitro-in vivo correlation data for fluconazole, itraconazole, and candida infections. Subcommittee on Antifungal Susceptibility Testing of the National Committee for Clinical Laboratory Standards. Clin Infect Dis 24:235-247.

140. Rex JH, Pfaller MA, Lancaster M, Odds FC, Bolmstrom A, Rinaldi MG. 1996. Quality control guidelines for National Committee for Clinical Laboratory Standards--recommended broth macrodilution testing of ketoconazole and itraconazole. J Clin Microbiol 34:816-817.

141. Como JA, Dismukes WE. 1994. Oral azole drugs as systemic antifungal therapy. N Engl J Med 330:263-272.

142. Gallant JE, Moore RD, Chaisson RE. 1994. Prophylaxis for opportunistic infections in patients with HIV infection. Annals of internal medicine 120:932944.

143. Kelly SL, Lamb DC, Kelly DE, Loeffler J, Einsele H. 1996. Resistance to fluconazole and amphotericin in Candida albicans from AIDS patients. Lancet 348:1523-1524.

144. Morschhauser J. 2002. The genetic basis of fluconazole resistance development in Candida albicans. Biochim Biophys Acta 1587:240-248.

145. Law D, Moore CB, Wardle HM, Ganguli LA, Keaney MG, Denning DW. 1994. High prevalence of antifungal resistance in Candida spp. from patients with AIDS. J Antimicrob Chemother 34:659-668.

146. Goodman JL, Winston DJ, Greenfield RA, Chandrasekar PH, Fox B, Kaizer H, Shadduck RK, Shea TC, Stiff P, Friedman DJ, et al. 1992. A controlled trial of fluconazole to prevent fungal infections in patients undergoing bone marrow transplantation. N Engl J Med 326:845-851.

147. Slavin MA, Osborne B, Adams R, Levenstein MJ, Schoch HG, Feldman AR, Meyers JD, Bowden RA. 1995. Efficacy and safety of fluconazole prophylaxis for fungal infections after marrow transplantation--a prospective, randomized, double-blind study. J Infect Dis 171:1545-1552.

148. Pittet D, Monod M, Suter PM, Frenk E, Auckenthaler R. 1994. Candida colonization and subsequent infections in critically ill surgical patients. Annals of surgery 220:751-758.

149. Rex JH, Bennett JE, Sugar AM, Pappas PG, van der Horst CM, Edwards JE, Washburn RG, Scheld WM, Karchmer AW, Dine AP, et al. 1994. A randomized trial comparing fluconazole with amphotericin B for the treatment of candidemia in patients without neutropenia. Candidemia Study Group and the National Institute. N Engl J Med 331:1325-1330.

150. Sobel JD, Brooker D, Stein GE, Thomason JL, Wermeling DP, Bradley B, Weinstein L. 1995. Single oral dose fluconazole compared with conventional clotrimazole topical therapy of Candida vaginitis. Fluconazole Vaginitis Study Group. American journal of obstetrics and gynecology 172:1263-1268. 
151. Neely MN, Ghannoum MA. 2000. The exciting future of antifungal therapy. European journal of clinical microbiology \& infectious diseases : official publication of the European Society of Clinical Microbiology 19:897-914.

152. Franz R, Kelly SL, Lamb DC, Kelly DE, Ruhnke M, Morschhauser J. 1998. Multiple molecular mechanisms contribute to a stepwise development of fluconazole resistance in clinical Candida albicans strains. Antimicrob Agents Chemother 42:3065-3072.

153. Franz R, Ruhnke M, Morschhauser J. 1999. Molecular aspects of fluconazole resistance development in Candida albicans. Mycoses 42:453-458.

154. Lopez-Ribot JL, McAtee RK, Lee LN, Kirkpatrick WR, White TC, Sanglard D, Patterson TF. 1998. Distinct patterns of gene expression associated with development of fluconazole resistance in serial candida albicans isolates from human immunodeficiency virus-infected patients with oropharyngeal candidiasis. Antimicrob Agents Chemother 42:2932-2937.

155. Sanglard D, Kuchler K, Ischer F, Pagani JL, Monod M, Bille J. 1995. Mechanisms of resistance to azole antifungal agents in Candida albicans isolates from AIDS patients involve specific multidrug transporters. Antimicrob Agents Chemother 39:2378-2386.

156. White TC. 1997. Increased mRNA levels of ERG16, CDR, and MDR1 correlate with increases in azole resistance in Candida albicans isolates from a patient infected with human immunodeficiency virus. Antimicrob Agents Chemother 41:1482-1487.

157. Nakamura K, Niimi M, Niimi K, Holmes AR, Yates JE, Decottignies A, Monk BC, Goffeau A, Cannon RD. 2001. Functional expression of Candida albicans drug efflux pump Cdr1p in a Saccharomyces cerevisiae strain deficient in membrane transporters. Antimicrob Agents Chemother 45:3366-3374.

158. Lyons CN, White TC. 2000. Transcriptional analyses of antifungal drug resistance in Candida albicans. Antimicrob Agents Chemother 44:2296-2303.

159. Niimi M, Niimi K, Takano Y, Holmes AR, Fischer FJ, Uehara Y, Cannon RD. 2004. Regulated overexpression of CDR1 in Candida albicans confers multidrug resistance. J Antimicrob Chemother 54:999-1006.

160. Tsao S, Rahkhoodaee F, Raymond M. 2009. Relative contributions of the Candida albicans ABC transporters Cdr1p and Cdr2p to clinical azole resistance. Antimicrob Agents Chemother 53:1344-1352.

161. Kohli A, Gupta V, Krishnamurthy S, Hasnain SE, Prasad R. 2001. Specificity of drug transport mediated by CaMDR1: a major facilitator of Candida albicans. Journal of biosciences 26:333-339.

162. Hiller D, Sanglard D, Morschhauser J. 2006. Overexpression of the MDR1 gene is sufficient to confer increased resistance to toxic compounds in Candida albicans. Antimicrob Agents Chemother 50:1365-1371.

163. Nolte FS, Parkinson T, Falconer DJ, Dix S, Williams J, Gilmore C, Geller R, Wingard JR. 1997. Isolation and characterization of fluconazole- and amphotericin B-resistant Candida albicans from blood of two patients with leukemia. Antimicrob Agents Chemother 41:196-199. 
164. Redding S, Smith J, Farinacci G, Rinaldi M, Fothergill A, Rhine-Chalberg J, Pfaller M. 1994. Resistance of Candida albicans to fluconazole during treatment of oropharyngeal candidiasis in a patient with AIDS: documentation by in vitro susceptibility testing and DNA subtype analysis. Clin Infect Dis 18:240-242.

165. White TC. 1997. The presence of an R467K amino acid substitution and loss of allelic variation correlate with an azole-resistant lanosterol 14alpha demethylase in Candida albicans. Antimicrob Agents Chemother 41:1488-1494.

166. Sanglard D, Ischer F, Koymans L, Bille J. 1998. Amino acid substitutions in the cytochrome P-450 lanosterol 14alpha-demethylase (CYP51A1) from azoleresistant Candida albicans clinical isolates contribute to resistance to azole antifungal agents. Antimicrob Agents Chemother 42:241-253.

167. Perea S, Lopez-Ribot JL, Kirkpatrick WR, McAtee RK, Santillan RA, Martinez M, Calabrese D, Sanglard D, Patterson TF. 2001. Prevalence of molecular mechanisms of resistance to azole antifungal agents in Candida albicans strains displaying high-level fluconazole resistance isolated from human immunodeficiency virus-infected patients. Antimicrob Agents Chemother 45:2676-2684.

168. Rogers PD, Barker KS. 2003. Genome-wide expression profile analysis reveals coordinately regulated genes associated with stepwise acquisition of azole resistance in Candida albicans clinical isolates. Antimicrob Agents Chemother 47:1220-1227.

169. Sanglard D, Ischer F, Monod M, Bille J. 1996. Susceptibilities of Candida albicans multidrug transporter mutants to various antifungal agents and other metabolic inhibitors. Antimicrob Agents Chemother 40:2300-2305.

170. Vinson C, Myakishev M, Acharya A, Mir AA, Moll JR, Bonovich M. 2002. Classification of human B-ZIP proteins based on dimerization properties. Mol Cell Biol 22:6321-6335.

171. Chinenov Y, Kerppola TK. 2001. Close encounters of many kinds: Fos-Jun interactions that mediate transcription regulatory specificity. Oncogene 20:24382452.

172. Moye-Rowley WS, Harshman KD, Parker CS. 1989. Yeast YAP1 encodes a novel form of the jun family of transcriptional activator proteins. Genes \& development 3:283-292.

173. Alarco AM, Balan I, Talibi D, Mainville N, Raymond M. 1997. AP1-mediated multidrug resistance in Saccharomyces cerevisiae requires FLR1 encoding a transporter of the major facilitator superfamily. J Biol Chem 272:19304-19313.

174. Toone WM, Morgan BA, Jones N. 2001. Redox control of AP-1-like factors in yeast and beyond. Oncogene 20:2336-2346.

175. MacPherson S, Larochelle M, Turcotte B. 2006. A fungal family of transcriptional regulators: the zinc cluster proteins. Microbiol Mol Biol Rev 70:583-604.

176. Akache B, Wu K, Turcotte B. 2001. Phenotypic analysis of genes encoding yeast zinc cluster proteins. Nucleic acids research 29:2181-2190.

177. Akache B, Turcotte B. 2002. New regulators of drug sensitivity in the family of yeast zinc cluster proteins. J Biol Chem 277:21254-21260. 
178. Reece RJ, Ptashne M. 1993. Determinants of binding-site specificity among yeast C6 zinc cluster proteins. Science 261:909-911.

179. Schjerling P, Holmberg S. 1996. Comparative amino acid sequence analysis of the C6 zinc cluster family of transcriptional regulators. Nucleic acids research 24:4599-4607.

180. Zhou KM, Bai YL, Kohlhaw GB. 1990. Yeast regulatory protein LEU3: a structure-function analysis. Nucleic acids research 18:291-298.

181. Leng P, Lee PR, Wu H, Brown AJ. 2001. Efg1, a morphogenetic regulator in Candida albicans, is a sequence-specific DNA binding protein. Journal of bacteriology 183:4090-4093.

182. Tebarth B, Doedt T, Krishnamurthy S, Weide M, Monterola F, Dominguez A, Ernst JF. 2003. Adaptation of the Efglp morphogenetic pathway in Candida albicans by negative autoregulation and PKA-dependent repression of the EFG1 gene. Journal of molecular biology 329:949-962.

183. Sohn K, Urban C, Brunner H, Rupp S. 2003. EFG1 is a major regulator of cell wall dynamics in Candida albicans as revealed by DNA microarrays. Mol Microbiol 47:89-102.

184. Prasad T, Hameed S, Manoharlal R, Biswas S, Mukhopadhyay CK, Goswami SK, Prasad R. 2010. Morphogenic regulator EFG1 affects the drug susceptibilities of pathogenic Candida albicans. FEMS yeast research 10:587-596.

185. Santos M, de Larrinoa IF. 2005. Functional characterization of the Candida albicans CRZ1 gene encoding a calcineurin-regulated transcription factor. Current genetics 48:88-100.

186. Bruno VM, Mitchell AP. 2005. Regulation of azole drug susceptibility by Candida albicans protein kinase CK2. Mol Microbiol 56:559-573.

187. Homann OR, Dea J, Noble SM, Johnson AD. 2009. A phenotypic profile of the Candida albicans regulatory network. PLoS genetics 5:e1000783.

188. Toone WM, Jones N. 1999. AP-1 transcription factors in yeast. Current opinion in genetics \& development 9:55-61.

189. Alarco AM, Raymond M. 1999. The bZip transcription factor Cap1p is involved in multidrug resistance and oxidative stress response in Candida albicans. Journal of bacteriology 181:700-708.

190. Kusch H, Engelmann S, Albrecht D, Morschhauser J, Hecker M. 2007. Proteomic analysis of the oxidative stress response in Candida albicans. Proteomics 7:686-697.

191. Wang Y, Cao YY, Cao YB, Wang DJ, Jia XM, Fu XP, Zhang JD, Xu Z, Ying K, Chen WS, Jiang YY. 2007. Cap1p plays regulation roles in redox, energy metabolism and substance transport: an investigation on Candida albicans under normal culture condition. Frontiers in bioscience : a journal and virtual library 12:145-153.

192. Znaidi S, Barker KS, Weber S, Alarco AM, Liu TT, Boucher G, Rogers PD, Raymond M. 2009. Identification of the Candida albicans Cap1p regulon. Eukaryot Cell 8:806-820.

193. Harry JB, Oliver BG, Song JL, Silver PM, Little JT, Choiniere J, White TC. 2005. Drug-induced regulation of the MDR1 promoter in Candida albicans. Antimicrob Agents Chemother 49:2785-2792. 
194. Hiller D, Stahl S, Morschhauser J. 2006. Multiple cis-acting sequences mediate upregulation of the MDR1 efflux pump in a fluconazole-resistant clinical Candida albicans isolate. Antimicrob Agents Chemother 50:2300-2308.

195. Morschhauser J, Barker KS, Liu TT, Bla BWJ, Homayouni R, Rogers PD. 2007. The transcription factor Mrrlp controls expression of the MDR1 efflux pump and mediates multidrug resistance in Candida albicans. PLoS Pathog 3:e164.

196. Coste AT, Karababa M, Ischer F, Bille J, Sanglard D. 2004. TAC1, transcriptional activator of CDR genes, is a new transcription factor involved in the regulation of Candida albicans $\mathrm{ABC}$ transporters CDR1 and CDR2. Eukaryot Cell 3:1639-1652.

197. de Micheli M, Bille J, Schueller C, Sanglard D. 2002. A common drugresponsive element mediates the upregulation of the Candida albicans $\mathrm{ABC}$ transporters CDR1 and CDR2, two genes involved in antifungal drug resistance. Mol Microbiol 43:1197-1214.

198. Coste A, Turner V, Ischer F, Morschhauser J, Forche A, Selmecki A, Berman J, Bille J, Sanglard D. 2006. A mutation in Tac1p, a transcription factor regulating CDR1 and CDR2, is coupled with loss of heterozygosity at chromosome 5 to mediate antifungal resistance in Candida albicans. Genetics 172:2139-2156.

199. MacPherson S, Akache B, Weber S, De Deken X, Raymond M, Turcotte B. 2005. Candida albicans zinc cluster protein Upc2p confers resistance to antifungal drugs and is an activator of ergosterol biosynthetic genes. Antimicrob Agents Chemother 49:1745-1752.

200. Silver PM, Oliver BG, White TC. 2004. Role of Candida albicans transcription factor Upc2p in drug resistance and sterol metabolism. Eukaryot Cell 3:13911397.

201. Znaidi S, Weber S, Al-Abdin OZ, Bomme P, Saidane S, Drouin S, Lemieux S, De Deken X, Robert F, Raymond M. 2008. Genomewide location analysis of Candida albicans Upc2p, a regulator of sterol metabolism and azole drug resistance. Eukaryot Cell 7:836-847.

202. Dunkel N, Liu TT, Barker KS, Homayouni R, Morschhauser J, Rogers PD. 2008. A gain-of-function mutation in the transcription factor Upc2p causes upregulation of ergosterol biosynthesis genes and increased fluconazole resistance in a clinical Candida albicans isolate. Eukaryot Cell 7:1180-1190.

203. Heilmann CJ, Schneider S, Barker KS, Rogers PD, Morschhauser J. 2010. An A643T mutation in the transcription factor Upc2p causes constitutive ERG11 upregulation and increased fluconazole resistance in Candida albicans. Antimicrob Agents Chemother 54:353-359.

204. Hoot SJ, Smith AR, Brown RP, White TC. 2011. An A643V amino acid substitution in Upc2p contributes to azole resistance in well-characterized clinical isolates of Candida albicans. Antimicrob Agents Chemother 55:940-942.

205. Flowers SA, Barker KS, Berkow EL, Toner G, Chadwick SG, Gygax SE, Morschhauser J, Rogers PD. 2012. Gain-of-function mutations in UPC2 are a frequent cause of ERG11 upregulation in azole-resistant clinical isolates of Candida albicans. Eukaryot Cell 11:1289-1299. 
206. Pfaller MA, Sheehan DJ, Rex JH. 2004. Determination of fungicidal activities against yeasts and molds: lessons learned from bactericidal testing and the need for standardization. Clin Microbiol Rev 17:268-280.

207. Sussman A, Huss K, Chio LC, Heidler S, Shaw M, Ma D, Zhu G, Campbell RM, Park TS, Kulanthaivel P, Scott JE, Carpenter JW, Strege MA, Belvo MD, Swartling JR, Fischl A, Yeh WK, Shih C, Ye XS. 2004. Discovery of cercosporamide, a known antifungal natural product, as a selective Pkc1 kinase inhibitor through high-throughput screening. Eukaryot Cell 3:932-943.

208. Jarvis WR. 1995. Epidemiology of nosocomial fungal infections, with emphasis on Candida species. Clin Infect Dis 20:1526-1530.

209. Gudlaugsson O, Gillespie S, Lee K, Vande Berg J, Hu J, Messer S, Herwaldt L, Pfaller M, Diekema D. 2003. Attributable mortality of nosocomial candidemia, revisited. Clin Infect Dis 37:1172-1177.

210. Silverman S, Jr., Gallo JW, McKnight ML, Mayer P, deSanz S, Tan MM. 1996. Clinical characteristics and management responses in 85 HIV-infected patients with oral candidiasis. Oral Surg Oral Med Oral Pathol Oral Radiol Endod 82:402-407.

211. Kontoyiannis DP, Lewis RE. 2002. Antifungal drug resistance of pathogenic fungi. Lancet 359:1135-1144.

212. Traeder C, Kowoll S, Arasteh K. 2008. Candida infection in HIV positive patients 1985-2007. Mycoses 51 Suppl 2:58-61.

213. Hitchcock CA, Whittle PJ. 1993. Chemistry and mode of action of fluconazole, p. 183-197. In Rippon JW, Fromtling RA (ed.), Cutaneous antifungal agents: selected compounds in clinical practice and development. Marcel Dekker, Inc., New York, N.Y.

214. Schubert S, Barker KS, Znaidi S, Schneider S, Dierolf F, Dunkel N, Aid M, Boucher G, Rogers PD, Raymond M, Morschhauser J. 2011. Regulation of efflux pump expression and drug resistance by the transcription factors Mrr1, Upc2, and Cap1 in Candida albicans. Antimicrob Agents Chemother 55:22122223.

215. Klepser ME, Wolfe EJ, Jones RN, Nightingale CH, Pfaller MA. 1997. Antifungal pharmacodynamic characteristics of fluconazole and amphotericin B tested against Candida albicans. Antimicrobial Agents and Chemotherapy 41:1392-1395.

216. Dunkel N, Liu TT, Barker KS, Homayouni R, Morschhauser J, Rogers PD. 2008. A gain-of-function mutation in the transcription factor Upc2p causes Upregulation of ergosterol biosynthesis genes and increased Fluconazole resistance in a clinical Candida albicans isolate. Eukaryotic Cell 7:1180-1190.

217. Amberg DC BD, Strathern JN. 2006. Isolation of yeast genomic DNA for southern blot analysis. CSH Protoc. 2006:pii: pdb.prot4149. doi: 4110.1101/pdb.prot4149.

218. Schmitt ME, Brown TA, Trumpower BL. 1990. A Rapid and Simple Method for Preparation of Rna from Saccharomyces-Cerevisiae. Nucleic Acids Research 18:3091-3092. 
219. Flowers SA, Barker KS, Berkow EL, Toner G, Chadwick SG, Gygax SE, Morschhauser J, Rogers PD. 2012. Gain-of-Function Mutations in UPC2 Are a Frequent Cause of ERG11 Upregulation in Azole-Resistant Clinical Isolates of Candida albicans. Eukaryotic Cell 11:1289-1299.

220. Sasse C, Schillig R, Dierolf F, Weyler M, Schneider S, Mogavero S, Rogers PD, Morschhauser J. 2011. The transcription factor Ndt80 does not contribute to Mrr1-, Tac1-, and Upc2-mediated fluconazole resistance in Candida albicans. PloS one 6:e25623.

221. Schubert S, Popp C, Rogers PD, Morschhauser J. 2011. Functional dissection of a Candida albicans zinc cluster transcription factor, the multidrug resistance regulator Mrr1. Eukaryot Cell 10:1110-1121.

222. Marichal P, Koymans L, Willemsens S, Bellens D, Verhasselt P, Luyten W, Borgers M, Ramaekers FC, Odds FC, Bossche HV. 1999. Contribution of mutations in the cytochrome P450 14alpha-demethylase (Erg11p, Cyp51p) to azole resistance in Candida albicans. Microbiology 145 ( Pt 10):2701-2713.

223. White TC, Holleman S, Dy F, Mirels LF, Stevens DA. 2002. Resistance mechanisms in clinical isolates of Candida albicans. Antimicrobial Agents and Chemotherapy 46:1704-1713.

224. Oliver BG, Song JL, Choiniere JH, White TC. 2007. cis-Acting elements within the Candida albicans ERG11 promoter mediate the azole response through transcription factor Upc2p. Eukaryot Cell 6:2231-2239.

225. Sasse C, Dunkel N, Schafer T, Schneider S, Dierolf F, Ohlsen K, Morschhauser J. 2012. The stepwise acquisition of fluconazole resistance mutations causes a gradual loss of fitness in Candida albicans. Mol Microbiol 86:539-556.

226. Kelly SL, Lamb DC, Kelly DE, Manning NJ, Loeffler J, Hebart H, Schumacher U, Einsele H. 1997. Resistance to fluconazole and cross-resistance to amphotericin B in Candida albicans from AIDS patients caused by defective sterol Delta(5,6)-desaturation. Febs Letters 400:80-82.

227. Henry KW, Nickels JT, Edlind TD. 2000. Upregulation of ERG genes in Candida species by azoles and other sterol biosynthesis inhibitors. Antimicrob Agents Chemother 44:2693-2700.

228. Prasad T, Chandra A, Mukhopadhyay CK, Prasad R. 2006. Unexpected link between iron and drug resistance of Candida spp.: iron depletion enhances membrane fluidity and drug diffusion, leading to drug-susceptible cells. Antimicrob Agents Chemother 50:3597-3606.

229. Kuipers ME, Beljaars L, Van Beek N, De Vries HG, Heegsma J, Van Den Berg JJ, Meijer DK, Swart PJ. 2002. Conditions influencing the in vitro antifungal activity of lactoferrin combined with antimycotics against clinical isolates of Candida. Impact on the development of buccal preparations of lactoferrin. APMIS : acta pathologica, microbiologica, et immunologica Scandinavica 110:290-298.

230. Kuipers ME, de Vries HG, Eikelboom MC, Meijer DK, Swart PJ. 1999. Synergistic fungistatic effects of lactoferrin in combination with antifungal drugs against clinical Candida isolates. Antimicrob Agents Chemother 43:2635-2641. 
231. Venkatesh MP, Rong L. 2008. Human recombinant lactoferrin acts synergistically with antimicrobials commonly used in neonatal practice against coagulase-negative staphylococci and Candida albicans causing neonatal sepsis. Journal of medical microbiology 57:1113-1121.

232. Martel CM, Parker JE, Bader O, Weig M, Gross U, Warrilow AG, Rolley N, Kelly DE, Kelly SL. 2010. Identification and characterization of four azoleresistant erg3 mutants of Candida albicans. Antimicrob Agents Chemother 54:4527-4533.

233. Kim J, Cho YJ, Do E, Choi J, Hu G, Cadieux B, Chun J, Lee Y, Kronstad JW, Jung WH. 2012. A defect in iron uptake enhances the susceptibility of Cryptococcus neoformans to azole antifungal drugs. Fungal genetics and biology: FG \& B 49:955-966.

234. Vale-Silva LA, Coste AT, Ischer F, Parker JE, Kelly SL, Pinto E, Sanglard D. 2012. Azole resistance by loss of function of the sterol Delta(5),(6)-desaturase gene (ERG3) in Candida albicans does not necessarily decrease virulence. Antimicrob Agents Chemother 56:1960-1968.

235. Barker KS, Crisp S, Wiederhold N, Lewis RE, Bareither B, Eckstein J, Barbuch R, Bard M, Rogers PD. 2004. Genome-wide expression profiling reveals genes associated with amphotericin $\mathrm{B}$ and fluconazole resistance in experimentally induced antifungal resistant isolates of Candida albicans. J Antimicrob Chemother 54:376-385.

236. Liu TT, Lee RE, Barker KS, Lee RE, Wei L, Homayouni R, Rogers PD. 2005. Genome-wide expression profiling of the response to azole, polyene, echinocandin, and pyrimidine antifungal agents in Candida albicans. Antimicrob Agents Chemother 49:2226-2236.

237. Reinoso-Martin C, Schuller C, Schuetzer-Muehlbauer M, Kuchler K. 2003. The yeast protein kinase $\mathrm{C}$ cell integrity pathway mediates tolerance to the antifungal drug caspofungin through activation of Slt $2 p$ mitogen-activated protein kinase signaling. Eukaryot Cell 2:1200-1210.

238. Klepser ME, Wolfe EJ, Jones RN, Nightingale CH, Pfaller MA. 1997. Antifungal pharmacodynamic characteristics of fluconazole and amphotericin B tested against Candida albicans. Antimicrob Agents Chemother 41:1392-1395.

239. Cid VJ, Duran A, del Rey F, Snyder MP, Nombela C, Sanchez M. 1995. Molecular basis of cell integrity and morphogenesis in Saccharomyces cerevisiae. Microbiol Rev 59:345-386.

240. Navarro-Garcia F, Sanchez M, Pla J, Nombela C. 1995. Functional characterization of the MKC1 gene of Candida albicans, which encodes a mitogen-activated protein kinase homolog related to cell integrity. Mol Cell Biol 15:2197-2206.

241. Navarro-Garcia F, Eisman B, Fiuza SM, Nombela C, Pla J. 2005. The MAP kinase Mkclp is activated under different stress conditions in Candida albicans. Microbiology 151:2737-2749.

242. White TC, Holleman S, Dy F, Mirels LF, Stevens DA. 2002. Resistance mechanisms in clinical isolates of Candida albicans. Antimicrob Agents Chemother 46: 1704-1713. 
243. Sanglard D, Ischer F, Parkinson T, Falconer D, Bille J. 2003. Candida albicans mutations in the ergosterol biosynthetic pathway and resistance to several antifungal agents. Antimicrob Agents Chemother 47:2404-2412.

244. Epp E, Vanier G, Harcus D, Lee AY, Jansen G, Hallett M, Sheppard DC, Thomas DY, Munro CA, Mullick A, Whiteway M. 2010. Reverse genetics in Candida albicans predicts ARF cycling is essential for drug resistance and virulence. PLoS Pathog 6:e1000753.

245. Heilmann CJ, Sorgo AG, Mohammadi S, Sosinska GJ, de Koster CG, Brul S, de Koning LJ, Klis FM. 2013. Surface stress induces a conserved cell wall stress response in the pathogenic fungus Candida albicans. Eukaryot Cell 12:254-264.

246. Edlind T. 2001. 41st ICAAC, Chicago, IL.

247. Klis FM, Sosinska GJ, de Groot PW, Brul S. 2009. Covalently linked cell wall proteins of Candida albicans and their role in fitness and virulence. FEMS yeast research 9:1013-1028.

248. Aaron KE, Pierson CA, Lees ND, Bard M. 2001. The Candida albicans ERG26 gene encoding the $\mathrm{C}-3$ sterol dehydrogenase (C-4 decarboxylase) is essential for growth. FEMS yeast research 1:93-101.

249. Ramanan N, Wang Y. 2000. A high-affinity iron permease essential for Candida albicans virulence. Science 288:1062-1064.

250. de Silva D, Davis-Kaplan S, Fergestad J, Kaplan J. 1997. Purification and characterization of Fet3 protein, a yeast homologue of ceruloplasmin. J Biol Chem 272:14208-14213.

251. Eck R, Hundt S, Hartl A, Roemer E, Kunkel W. 1999. A multicopper oxidase gene from Candida albicans: cloning, characterization and disruption. Microbiology 145 ( Pt 9):2415-2422.

252. Robertson LS, Causton HC, Young RA, Fink GR. 2000. The yeast A kinases differentially regulate iron uptake and respiratory function. Proc Natl Acad Sci U S A 97:5984-5988.

253. Hameed S, Dhamgaye S, Singh A, Goswami SK, Prasad R. 2011. Calcineurin signaling and membrane lipid homeostasis regulates iron mediated multidrug resistance mechanisms in Candida albicans. PloS one 6:e18684.

254. Lan CY, Rodarte G, Murillo LA, Jones T, Davis RW, Dungan J, Newport G, Agabian N. 2004. Regulatory networks affected by iron availability in Candida albicans. Mol Microbiol 53:1451-1469.

255. Li L, Kaplan J. 1996. Characterization of yeast methyl sterol oxidase (ERG25) and identification of a human homologue. J Biol Chem 271:16927-16933.

256. Pierson CA, Eckstein J, Barbuch R, Bard M. 2004. Ergosterol gene expression in wild-type and ergosterol-deficient mutants of Candida albicans. Med Mycol 42:385-389.

257. Dhamgaye S, Devaux F, Manoharlal R, Vandeputte P, Shah AH, Singh A, Blugeon C, Sanglard D, Prasad R. 2012. In vitro effect of malachite green on Candida albicans involves multiple pathways and transcriptional regulators UPC2 and STP2. Antimicrob Agents Chemother 56:495-506. 
258. Sorgo AG, Heilmann CJ, Dekker HL, Bekker M, Brul S, de Koster CG, de Koning LJ, Klis FM. 2011. Effects of fluconazole on the secretome, the wall proteome, and wall integrity of the clinical fungus Candida albicans. Eukaryot Cell 10:1071-1081.

259. Castillo L, Martinez AI, Garcera A, Garcia-Martinez J, Ruiz-Herrera J, Valentin E, Sentandreu R. 2006. Genomic response programs of Candida albicans following protoplasting and regeneration. Fungal genetics and biology : FG \& B 43:124-134.

260. Gelis S, de Groot PW, Castillo L, Moragues MD, Sentandreu R, Gomez MM, Valentin E. 2012. Pga13 in Candida albicans is localized in the cell wall and influences cell surface properties, morphogenesis and virulence. Fungal genetics and biology : FG \& B 49:322-331.

261. Cornet M, Gaillardin C, Richard ML. 2006. Deletions of the endocytic components VPS28 and VPS32 in Candida albicans lead to echinocandin and azole hypersensitivity. Antimicrob Agents Chemother 50:3492-3495.

262. Niewerth M, Kunze D, Seibold M, Schaller M, Korting HC, Hube B. 2003. Ciclopirox olamine treatment affects the expression pattern of Candida albicans genes encoding virulence factors, iron metabolism proteins, and drug resistance factors. Antimicrob Agents Chemother 47:1805-1817.

263. Abrams BB, Hanel H, Hoehler T. 1991. Ciclopirox olamine: a hydroxypyridone antifungal agent. Clinics in dermatology 9:471-477.

264. Sakurai K, Sakaguchi T, Yamaguchi H, Iwata K. 1978. Studies on uptake of 6cyclohexyl-1-hydroxy-4-methyl-2(1H)-pyridone ethanolamine salt (Hoe 296) by Candida albicans. Chemotherapy 24:146-153.

265. Lee RE, Liu TT, Barker KS, Lee RE, Rogers PD. 2005. Genome-wide expression profiling of the response to ciclopirox olamine in Candida albicans. J Antimicrob Chemother 55:655-662.

266. Fiori A, Van Dijck P. 2012. Potent synergistic effect of doxycycline with fluconazole against Candida albicans is mediated by interference with iron homeostasis. Antimicrob Agents Chemother 56:3785-3796.

267. Nelson ML. 1998. Chemical and biological dynamics of tetracyclines. Advances in dental research 12:5-11.

268. Grenier D, Huot MP, Mayrand D. 2000. Iron-chelating activity of tetracyclines and its impact on the susceptibility of Actinobacillus actinomycetemcomitans to these antibiotics. Antimicrob Agents Chemother 44:763-766.

269. Ryan ME, Usman A, Ramamurthy NS, Golub LM, Greenwald RA. 2001. Excessive matrix metalloproteinase activity in diabetes: inhibition by tetracycline analogues with zinc reactivity. Current medicinal chemistry 8:305-316.

270. Griffin MO, Ceballos G, Villarreal FJ. 2011. Tetracycline compounds with non-antimicrobial organ protective properties: possible mechanisms of action. Pharmacological research : the official journal of the Italian Pharmacological Society 63:102-107.

271. Selmecki A, Gerami-Nejad M, Paulson C, Forche A, Berman J. 2008. An isochromosome confers drug resistance in vivo by amplification of two genes, ERG11 and TAC1. Mol Microbiol 68:624-641. 
272. Yoshida Y, Aoyama Y. 1987. Interaction of azole antifungal agents with cytochrome P-45014DM purified from Saccharomyces cerevisiae microsomes. Biochem Pharmacol 36:229-235. 
APPENDIX A. UPC2 SUPPLEMENTAL DATA

Table A-1. Genes upregulated by at least 1.5-fold by fluconazole in SC5314

\begin{tabular}{|c|c|c|c|c|c|c|c|c|}
\hline \multirow[b]{3}{*}{$\begin{array}{c}\text { orf19 } \\
\text { Designation }\end{array}$} & \multirow[b]{3}{*}{$\begin{array}{c}\text { CGD } \\
\text { Name }\end{array}$} & \multirow[b]{3}{*}{ Process $^{a}$} & \multicolumn{6}{|c|}{ Change in Fold Expression } \\
\hline & & & \multicolumn{2}{|c|}{$\begin{array}{c}\text { SC5314+FLC } \\
\text { /SC5314 } \\
\end{array}$} & \multicolumn{2}{|c|}{$\begin{array}{c}\operatorname{cas} 5 \Delta / \Delta+\mathrm{FLC} \\
/ \operatorname{cas} 5 \Delta / \Delta \\
\end{array}$} & \multicolumn{2}{|c|}{$\begin{array}{c}u p c 2 \Delta / \Delta+\mathrm{FLC} \\
u p c 2 \Delta / \Delta\end{array}$} \\
\hline & & & Exp1 & Exp2 & Exp1 & Exp2 & Exp1 & Exp2 \\
\hline orf19.7094 & HGT12 & carbohydrate transport & 423.2 & 19.8 & 8.0 & 1.9 & 13.5 & 140.0 \\
\hline orf19.4335 & $T N A 1$ & anion transport & 194.9 & 29.8 & 2.5 & 42.4 & 0.4 & 0.2 \\
\hline orf19.6084 & & & 123.7 & 19.7 & 11.8 & 9.8 & 62.0 & 51.5 \\
\hline orf19.4527 & $H G T 1$ & carbohydrate transport & 115.9 & 120.9 & 98.6 & 141.2 & 59.1 & 88.7 \\
\hline orf19.4476 & & cellular aldehyde metabolic process & 104.5 & 102.1 & 114.2 & 83.9 & 419.7 & 228.8 \\
\hline orf19.3499 & & & 79.5 & 20.5 & 5.9 & 7.6 & 10.6 & 10.1 \\
\hline orf19.3923 & $P G A 37$ & & 73.2 & 6.3 & 7.7 & 22.1 & 91.4 & 58.4 \\
\hline orf19.6311 & & & 72.8 & 23.5 & 382.3 & 60.5 & 98.1 & 13.7 \\
\hline orf19.4384 & HXT5 & high-affinity hexose transport & 70.6 & 75.5 & 11.1 & 23.4 & 16.9 & 9.7 \\
\hline orf19.5741 & $A L S 1$ & cell adhesion & 66.8 & 79.0 & 22.6 & 21.0 & 4.8 & 3.1 \\
\hline orf19.3908 & & & 51.0 & 14.6 & 5.4 & 9.0 & 512.3 & 140.7 \\
\hline orf19.3668 & $H G T 2$ & carbohydrate transport & 48.3 & 35.5 & 10.1 & 19.8 & 20.1 & 12.3 \\
\hline orf19.670.2 & & & 47.4 & 152.7 & 10.8 & 16.1 & 18.3 & 14.1 \\
\hline orf19.4682 & $H G T 17$ & carbohydrate transport & 45.0 & 24.7 & 7.0 & 7.3 & 4.2 & 4.5 \\
\hline orf19.1438 & & oxidation-reduction process & 42.4 & 13.5 & 36.3 & 29.8 & 17.4 & 9.8 \\
\hline orf19.2809 & CTN3 & acetyl-CoA metabolic process & 41.1 & 36.9 & 33.4 & 30.6 & 12.6 & 11.5 \\
\hline orf19.7093 & $H G T 13$ & carbohydrate transport & 40.9 & 18.8 & 5.9 & 11.3 & 9.8 & 2.6 \\
\hline orf19.7111.1 & SOD3 & response to oxidative stress & 40.5 & 69.2 & 9.4 & 15.0 & 46.5 & 99.8 \\
\hline orf19.7434 & $G L G 2$ & glycogen biosynthetic process & 37.5 & 16.1 & 7.5 & 8.7 & 47.7 & 39.9 \\
\hline orf19.1932 & CFL4 & siderophore-iron transport & 35.6 & 359.0 & 2.4 & 6.6 & 8.0 & 22.0 \\
\hline orf19.1473 & & oxidation-reduction process & 35.0 & 15.0 & 3.8 & 4.8 & 30.4 & 41.0 \\
\hline orf19.2060 & SOD5 & cellular copper ion homeostasis & 33.2 & 14.2 & 5.4 & 6.1 & 14.2 & 28.1 \\
\hline orf19.467 & & & 32.3 & 7.1 & 3.0 & 2.9 & 2.8 & 0.8 \\
\hline
\end{tabular}


Table A-1. (Continued)

\begin{tabular}{|c|c|c|c|c|c|c|c|c|}
\hline \multirow[b]{3}{*}{$\begin{array}{c}\text { orf19 } \\
\text { Designation }\end{array}$} & \multirow[b]{3}{*}{$\begin{array}{c}\text { CGD } \\
\text { Name }\end{array}$} & \multirow[b]{3}{*}{ Process $^{a}$} & \multicolumn{6}{|c|}{ Change in Fold Expression } \\
\hline & & & \multicolumn{2}{|c|}{$\begin{array}{c}\text { SC5314+FLC } \\
\text { /SC5314 } \\
\end{array}$} & \multicolumn{2}{|c|}{$\begin{array}{c}\operatorname{cas} 5 \Delta / \Delta+\mathrm{FLC} \\
/ \operatorname{cas} 5 \Delta / \Delta\end{array}$} & \multicolumn{2}{|c|}{$\begin{array}{c}u p c 2 \Delta / \Delta+\mathrm{FLC} / \\
u p c 2 \Delta / \Delta\end{array}$} \\
\hline & & & Exp1 & Exp2 & Exp1 & Exp2 & Exp1 & Exp2 \\
\hline orf19.6993 & $G A P 2$ & gamma-aminobutyric acid transport & 30.6 & 10.6 & 3.5 & 10.0 & 6.0 & 3.0 \\
\hline orf19.4214 & & & 30.5 & 19.5 & 0.5 & 1.2 & 0.3 & 12.5 \\
\hline orf19.6486 & $L D G 3$ & & 29.6 & 3.1 & 8.1 & 4.2 & 15.7 & 11.4 \\
\hline orf19.4773 & $A O X 2$ & aerobic respiration & 29.5 & 24.9 & 15.4 & 14.1 & 122.1 & 50.4 \\
\hline orf19.2652 & TEF4 & translational elongation & 27.8 & 15.2 & 15.3 & 25.5 & 9.5 & 8.5 \\
\hline orf19.1344 & & & 27.1 & 12.6 & 13.1 & 32.5 & 53.0 & 6.3 \\
\hline orf19.3232 & & anion transport & 24.6 & 5.3 & 2.6 & 1.0 & 1.6 & 1.6 \\
\hline orf19.4551 & CTN1 & acetyl-CoA metabolic process & 22.4 & 26.6 & 11.3 & 14.3 & 17.4 & 75.7 \\
\hline orf19.1655 & $P X P 2$ & fatty acid beta-oxidation & 22.1 & 31.9 & 19.5 & 19.9 & 95.6 & 41.4 \\
\hline orf19.3982 & & sucrose catabolic process & 21.1 & 5.4 & 2.5 & 3.2 & 44.9 & 44.0 \\
\hline orf19.2023 & $H G T 7$ & glucose transport & 20.8 & 21.6 & 9.4 & 7.8 & 0.5 & 0.4 \\
\hline orf19.5753 & HGT10 & carbohydrate transport & 20.6 & 1.6 & 2.9 & 2.9 & 2.6 & 0.8 \\
\hline orf19.6484 & & & 19.5 & 30.0 & 2.2 & 4.3 & 240.3 & 97.9 \\
\hline orf19.344 & & & 19.0 & 6.9 & 13.6 & 8.9 & 152.4 & 53.0 \\
\hline orf19.2810 & $A A P 1$ & amino acid transport & 18.9 & 19.6 & 7.5 & 8.8 & 32.9 & 13.3 \\
\hline orf19.6919 & & & 18.1 & 4.5 & 1.4 & 10.1 & 21.7 & 54.8 \\
\hline orf19.5302 & $P G A 31$ & fungal-type cell wall organization & 17.6 & 4.1 & 2.1 & 1.5 & 9.9 & 3.4 \\
\hline orf19.7197 & & DNA replication initiation & 17.0 & 6.6 & 2.0 & 7.3 & 11.6 & 22.6 \\
\hline orf19.3924 & & & 16.8 & 4.1 & 8.5 & 4.7 & 3.2 & 3.2 \\
\hline orf19.4691 & & & 16.6 & 24.8 & 4.2 & 3.4 & 4.0 & 59.6 \\
\hline orf19.4690 & & manganese ion transport & 16.3 & 20.2 & 7.8 & 23.4 & 1.0 & 1.4 \\
\hline orf19.6420 & $P G A 13$ & filamentous growth & 15.6 & 30.6 & 2.4 & 2.3 & 87.4 & 117.0 \\
\hline orf19.5069 & & meiotic DNA recombinase assembly & 14.9 & 15.5 & 5.9 & 7.1 & 28.3 & 16.1 \\
\hline orf19.740 & HAP41 & regulation of transcription from RNA polymerase II promoter & 14.3 & 10.7 & 12.7 & 23.5 & 14.3 & 2.5 \\
\hline orf19.5037 & $H R Q 2$ & & 13.5 & 9.7 & 8.4 & 12.3 & 14.4 & 4.8 \\
\hline orf19.7566 & & amino acid transport & 13.4 & 7.0 & 6.9 & 11.2 & 11.3 & 5.3 \\
\hline orf19.3627 & & & 12.6 & 5.5 & 10.1 & 10.8 & 26.6 & 13.5 \\
\hline
\end{tabular}


Table A-1. (Continued)

\begin{tabular}{|c|c|c|c|c|c|c|c|c|}
\hline \multirow[b]{3}{*}{$\begin{array}{c}\text { orf19 } \\
\text { Designation }\end{array}$} & \multirow[b]{3}{*}{$\begin{array}{l}\text { CGD } \\
\text { Name }\end{array}$} & \multirow[b]{3}{*}{ Process $^{\mathrm{a}}$} & \multicolumn{6}{|c|}{ Change in Fold Expression } \\
\hline & & & \multicolumn{2}{|c|}{$\begin{array}{c}\text { SC5314+FLC } \\
\text { /SC5314 }\end{array}$} & \multicolumn{2}{|c|}{$\begin{array}{l}\operatorname{cas} 5 \Delta / \Delta+F L C \\
/ \operatorname{cas} 5 \Delta / \Delta\end{array}$} & \multicolumn{2}{|c|}{$\begin{array}{c}u p c 2 \Delta / \Delta+\mathrm{FLC} / \\
u p c 2 \Delta / \Delta\end{array}$} \\
\hline & & & Exp1 & Exp2 & Exp1 & Exp2 & Exp1 & Exp2 \\
\hline orf19.893 & $P G A 17$ & & 12.3 & 5.5 & 1.4 & 1.1 & 32.2 & 3.5 \\
\hline orf19.6186 & & & 12.1 & 13.3 & 2.4 & 2.0 & 2.8 & 2.7 \\
\hline orf19.1765 & & & 11.7 & 7.6 & 4.4 & 6.9 & 22.8 & 13.6 \\
\hline orf19.638 & $F D H 1$ & formate metabolic process & 11.7 & 19.5 & 4.4 & 3.9 & 9.0 & 29.2 \\
\hline orf19.742 & ALD6 & ethanol metabolic process & 11.5 & 10.4 & 4.1 & 1.7 & 3.5 & 6.8 \\
\hline orf19.3986 & PPR1 & regulation of transcription, DNA-dependent & 10.9 & 5.3 & 9.9 & 10.3 & 10.7 & 3.2 \\
\hline orf19.7296 & & & 10.6 & 11.5 & 11.8 & 8.6 & 55.8 & 13.6 \\
\hline orf19.2717 & SAS10 & endonucleolytic cleavage of rRNA & 10.4 & 2.5 & 4.4 & 3.5 & 4.7 & 14.9 \\
\hline orf19.6595 & RTA4 & filamentous growth & 10.4 & 15.0 & 3.0 & 2.9 & 17.8 & 34.3 \\
\hline orf19.1763 & IFR1 & oxidation-reduction process & 10.3 & 11.8 & 15.9 & 7.9 & 53.4 & 50.8 \\
\hline orf19.5307 & JEN2 & lactate transport & 10.3 & 2.7 & 2.0 & 3.1 & 0.4 & 1.3 \\
\hline orf19.7504 & & & 10.1 & 4.9 & 12.6 & 25.3 & 23.1 & 5.3 \\
\hline orf19.6793 & & & 10.0 & 7.7 & 8.6 & 15.5 & 118.0 & 36.1 \\
\hline orf19.3733 & $I D P 2$ & glutamate biosynthetic process & 9.7 & 14.9 & 10.8 & 12.9 & 12.6 & 18.2 \\
\hline orf19.1363 & & ascospore formation & 9.5 & 29.7 & 11.0 & 10.3 & 70.4 & 1188.0 \\
\hline orf19.7585 & INOI & inositol biosynthetic process & 9.2 & 20.5 & 21.8 & 19.6 & 0.9 & 0.8 \\
\hline orf19.2049 & & & 9.1 & 5.6 & 8.5 & 5.2 & 59.6 & 21.2 \\
\hline orf19.10 & $A L K 8$ & fatty acid omega-oxidation & 9.0 & 8.1 & 3.0 & 3.5 & 133.9 & 183.6 \\
\hline orf19.5634 & FRP1 & high-affinity iron ion transport & 8.9 & 8.2 & 7.5 & 7.3 & 0.1 & 0.2 \\
\hline orf19.1070 & & & 8.6 & 8.0 & 5.4 & 4.6 & 2.4 & 1.6 \\
\hline orf19.4802 & FTH1 & high-affinity iron ion transport & 8.5 & 9.9 & 5.9 & 7.1 & 14.3 & 6.5 \\
\hline orf19.2946 & HNM4 & choline transport & 8.4 & 4.9 & 2.9 & 2.5 & 18.4 & 2.9 \\
\hline orf19.6844 & ICL1 & glyoxylate cycle & 8.2 & 8.2 & 17.3 & 23.3 & 17.4 & 50.8 \\
\hline orf19.4872 & & & 8.2 & 19.2 & 1.2 & 1.3 & 25.8 & 10.2 \\
\hline orf19.2108 & SOD6 & oxidation-reduction process & 7.9 & 10.6 & 1.6 & 1.1 & 1.1 & 1.6 \\
\hline orf19.1345 & LIP8 & lipid catabolic process & 7.9 & 6.2 & 1.3 & 4.3 & 107.5 & 3.9 \\
\hline orf19.5614 & & cell wall organization & 7.9 & 7.7 & 3.9 & 4.1 & 11.8 & 6.5 \\
\hline
\end{tabular}


Table A-1. (Continued)

\begin{tabular}{|c|c|c|c|c|c|c|c|c|}
\hline \multirow[b]{3}{*}{$\begin{array}{c}\text { orf19 } \\
\text { Designation }\end{array}$} & \multirow[b]{3}{*}{$\begin{array}{c}\text { CGD } \\
\text { Name }\end{array}$} & \multirow[b]{3}{*}{ Process $^{a}$} & \multicolumn{6}{|c|}{$\begin{array}{l}\text { Change in Fold Expression } \\
\end{array}$} \\
\hline & & & \multicolumn{2}{|c|}{$\begin{array}{c}\text { SC5314+FLC } \\
\text { /SC5314 } \\
\end{array}$} & \multicolumn{2}{|c|}{$\begin{array}{c}\operatorname{cas} 5 \Delta / \Delta+\mathrm{FLC} \\
/ \operatorname{cas} 5 \Delta / \Delta \\
\end{array}$} & \multicolumn{2}{|c|}{$\begin{array}{c}\text { upc } 2 \Delta / \Delta+\mathrm{FLC} / \\
u p c 2 \Delta / \Delta\end{array}$} \\
\hline & & & Exp1 & Exp2 & Exp1 & Exp2 & Exp1 & Exp2 \\
\hline orf19.3740 & $P G A 23$ & response to drug & 7.9 & 6.7 & 9.2 & 9.1 & 29.1 & 10.1 \\
\hline orf19.4706 & & & 7.7 & 6.2 & 15.1 & 12.7 & 17.5 & 6.6 \\
\hline orf19.5902 & $R A S 2$ & Ras protein signal transduction & 7.6 & 6.2 & 0.8 & 1.1 & 2.3 & 1.5 \\
\hline orf19.7314 & $C D G 1$ & L-cysteine metabolic process & 7.5 & 6.0 & 1.0 & 0.6 & 1.7 & 5.0 \\
\hline orf19.4404 & PGA49 & & 7.3 & 1.6 & 1.1 & 0.8 & 15.4 & 4.7 \\
\hline orf19.4608 & PDC12 & & 7.1 & 16.7 & 0.5 & 0.3 & 14.1 & 12.6 \\
\hline orf19.4274 & PUT1 & glutamate biosynthetic process & 6.8 & 8.8 & 15.1 & 21.1 & 2.5 & 0.6 \\
\hline orf19.94 & & & 6.8 & 6.6 & 17.5 & 25.8 & 12.3 & 6.2 \\
\hline orf19.1826 & MDM34 & regulation of transcription, DNA-dependent & 6.8 & 9.3 & 7.3 & 13.6 & 21.5 & 5.5 \\
\hline orf19.1277 & & & 6.6 & 9.3 & 1.5 & 1.5 & 3.4 & 2.7 \\
\hline orf19.5447 & HGT19 & carbohydrate transport & 6.6 & 8.1 & 7.8 & 18.5 & 9.8 & 3.9 \\
\hline orf19.6828 & & rRNA processing & 6.5 & 3.5 & 1.5 & 2.8 & 2.5 & 3.9 \\
\hline orf19.2747 & $R G T 1$ & filamentous growth & 6.4 & 4.6 & 5.3 & 43.8 & 97.4 & 10.4 \\
\hline orf19.7056 & & amino acid transmembrane transport & 6.2 & 120.3 & 2.5 & 2.7 & 94.2 & 9.2 \\
\hline orf19.7131 & & carnitine biosynthetic process & 6.2 & 6.8 & 6.2 & 7.8 & 3.6 & 1.8 \\
\hline orf19.5959 & NOP14 & response to drug & 6.1 & 4.0 & 5.3 & 4.6 & 9.0 & 7.7 \\
\hline orf19.3672 & GAL10 & fungal-type cell wall biogenesis & 6.0 & 7.3 & 14.2 & 6.9 & 2.7 & 3.8 \\
\hline orf19.5045 & PTP2 & osmosensory signaling pathway & 6.0 & 10.4 & 2.5 & 2.0 & 23.0 & 20.2 \\
\hline orf19.3615 & & & 6.0 & 5.4 & 8.2 & 8.1 & 12.8 & 10.9 \\
\hline orf19.2878 & $P G A 15$ & & 6.0 & 8.1 & 3.1 & 2.4 & 7.7 & 6.7 \\
\hline orf19.7514 & PCK1 & gluconeogenesis & 6.0 & 8.6 & 1.9 & 1.4 & 9.3 & 13.0 \\
\hline orf19.5542 & SAP6 & adhesion to host & 5.9 & 4.3 & 2.1 & 1.4 & 9.5 & 7.9 \\
\hline orf19.3684 & & ascospore formation & 5.9 & 2.1 & 11.2 & 11.3 & 9.1 & 15.4 \\
\hline orf19.2247 & & & 5.9 & 5.6 & 5.5 & 1.3 & 88.0 & 0.7 \\
\hline orf19.2515 & & & 5.9 & 9.0 & 6.7 & 7.1 & 13.4 & 7.9 \\
\hline orf19.2952 & $E X G 2$ & cell wall organization & 5.8 & 4.1 & 2.6 & 3.0 & 7.0 & 4.2 \\
\hline orf19.7077 & & iron ion transport & 5.8 & 5.5 & 4.1 & 4.1 & 90.1 & 64.1 \\
\hline
\end{tabular}


Table A-1. (Continued)

\begin{tabular}{|c|c|c|c|c|c|c|c|c|}
\hline \multirow[b]{3}{*}{$\begin{array}{c}\text { orf19 } \\
\text { Designation }\end{array}$} & \multirow[b]{3}{*}{$\begin{array}{c}\text { CGD } \\
\text { Name }\end{array}$} & \multirow[b]{3}{*}{ Process $^{a}$} & \multicolumn{6}{|c|}{ Change in Fold Expression } \\
\hline & & & \multicolumn{2}{|c|}{$\begin{array}{c}\text { SC5314+FLC } \\
\text { /SC5314 } \\
\end{array}$} & \multicolumn{2}{|c|}{$\begin{array}{c}\operatorname{cas} 5 \Delta / \Delta+\mathrm{FLC} \\
/ \operatorname{cas} 5 \Delta / \Delta \\
\end{array}$} & \multicolumn{2}{|c|}{$\begin{array}{c}\text { upc } 2 \Delta / \Delta+\mathrm{FLC} / \\
u p c 2 \Delta / \Delta\end{array}$} \\
\hline & & & Exp1 & Exp2 & Exp1 & Exp2 & Exp1 & Exp2 \\
\hline orf19.4082 & DDR 48 & hyphal growth & 5.8 & 5.7 & 9.3 & 6.9 & 1.9 & 1.3 \\
\hline orf19.1117 & & NADH regeneration & 5.7 & 2.3 & 0.7 & 1.1 & 1.3 & 2.0 \\
\hline orf19.1923 & $R R N 3$ & transcription from RNA polymerase I promoter & 5.7 & 23.0 & 2.9 & 3.8 & 13.4 & 3.5 \\
\hline orf19.1237 & ARO9 & aromatic amino acid family catabolic process to alcohol & 5.6 & 9.5 & 6.9 & 5.0 & 18.6 & 12.6 \\
\hline orf19.1069 & RPN4 & proteasomal ubiquitin-dependent protein catabolic process & 5.6 & 6.0 & 5.7 & 5.5 & 20.2 & 12.7 \\
\hline orf19.6920 & & & 5.6 & 1.9 & 7.3 & 6.0 & 31.0 & 20.1 \\
\hline orf19.410.3 & & riboflavin biosynthetic process & 5.6 & 5.7 & 0.6 & 0.5 & 1.0 & 0.8 \\
\hline orf19.3574 & MDJ2 & protein import into mitochondrial matrix & 5.6 & 3.1 & 2.0 & 1.2 & 2.1 & 3.6 \\
\hline orf19.4607 & & & 5.6 & 7.0 & 0.5 & 0.3 & 5.8 & 24.3 \\
\hline orf19.5000 & CYB2 & lactate metabolic process & 5.5 & 3.3 & 3.3 & 3.3 & 4.2 & 6.2 \\
\hline orf19.6249 & $H A K 1$ & potassium ion transport & 5.4 & 5.8 & 2.7 & 3.8 & 1.4 & 1.7 \\
\hline orf19.449 & & metabolic process & 5.4 & 5.6 & 5.2 & 5.9 & 3.4 & 1.6 \\
\hline orf19.3934 & CAR1 & arginine catabolic process to ornithine & 5.4 & 3.2 & 7.8 & 10.6 & 2.5 & 2.5 \\
\hline orf19.3547 & & regulation of transcription, mating-type specific & 5.3 & 5.9 & 1.1 & 1.7 & 2.7 & 3.8 \\
\hline orf19.918 & CDR11 & multidrug transport & 5.3 & 11.0 & 4.1 & 3.1 & 9.8 & 10.1 \\
\hline orf19.7263 & & cellular process & 5.3 & 6.0 & 5.0 & 4.5 & 2.6 & 2.2 \\
\hline orf19.5206 & & box H/ACA snoRNP assembly & 5.2 & 4.0 & 1.4 & 1.7 & 4.7 & 6.2 \\
\hline orf19.2292 & OPT4 & oligopeptide transport & 5.2 & 3.7 & 1.1 & 1.0 & 0.0 & 0.0 \\
\hline orf19.4273 & & aerobic respiration & 5.1 & 7.6 & 3.9 & 5.1 & 1.4 & 2.0 \\
\hline orf19.3931 & $S F C 1$ & fumarate transport & 5.1 & 5.2 & 9.9 & 13.9 & 6.9 & 3.4 \\
\hline orf19.6766 & NOP13 & & 5.1 & 5.3 & 1.7 & 2.6 & 4.7 & 9.8 \\
\hline orf19.3434 & TRY5 & positive regulation of cell-substrate adhesion & 5.0 & 7.3 & 6.8 & 7.5 & 6.9 & 3.1 \\
\hline orf19.4070 & & & 5.0 & 8.9 & 3.0 & 2.5 & 1.8 & 1.9 \\
\hline orf19.3670 & $G A L 1$ & galactose catabolic process & 5.0 & 8.2 & 12.9 & 7.4 & 4.7 & 2.7 \\
\hline orf19.5094 & BUL1 & protein polyubiquitination & 5.0 & 5.7 & 3.2 & 3.0 & 4.9 & 5.3 \\
\hline orf19.4658 & & & 5.0 & 6.6 & 2.5 & 3.1 & 2.4 & 1.1 \\
\hline orf19.5353 & & & 4.9 & 8.8 & 10.4 & 14.0 & 4.5 & 0.8 \\
\hline
\end{tabular}


Table A-1. (Continued)

\begin{tabular}{|c|c|c|c|c|c|c|c|c|}
\hline \multirow{3}{*}{$\begin{array}{c}\text { orf19 } \\
\text { Designation }\end{array}$} & \multirow{3}{*}{$\begin{array}{l}\text { CGD } \\
\text { Name }\end{array}$} & \multirow[b]{3}{*}{ Process $^{a}$} & \multicolumn{6}{|c|}{ Change in Fold Expression } \\
\hline & & & \multicolumn{2}{|c|}{$\begin{array}{c}\text { SC5314+FLC } \\
\text { /SC5314 } \\
\end{array}$} & \multicolumn{2}{|c|}{$\begin{array}{c}\operatorname{cas} 5 \Delta / \Delta+\mathrm{FLC} \\
/ \operatorname{cas} 5 \Delta / \Delta \\
\end{array}$} & \multicolumn{2}{|c|}{$\begin{array}{c}\text { upc } 2 \Delta / \Delta+\mathrm{FLC} / \\
u p c 2 \Delta / \Delta\end{array}$} \\
\hline & & & Exp1 & Exp2 & Exp1 & Exp2 & Exp1 & Exp2 \\
\hline orf19.4041 & PEX4 & protein autoubiquitination & 4.9 & 4.3 & 1.3 & 1.5 & 3.5 & 6.8 \\
\hline orf19.6886 & & maturation of rRNA & 4.9 & 4.9 & 2.5 & 4.6 & 16.5 & 16.7 \\
\hline orf19.5635 & $P G A 7$ & & 4.9 & 4.1 & 2.8 & 3.0 & 0.5 & 0.4 \\
\hline orf19.677 & $\mathrm{CHOl}$ & filamentous growth & 4.9 & 5.5 & 7.6 & 9.8 & 16.7 & 3.3 \\
\hline orf19.1377 & $I P K 2$ & arginine metabolic process & 4.9 & 3.4 & 4.6 & 7.4 & 16.4 & 13.3 \\
\hline orf19.7552 & & endonucleolytic cleavage of rRNA & 4.9 & 4.6 & 2.9 & 5.5 & 6.2 & 7.3 \\
\hline orf19.5716 & SAP4 & adhesion to host & 4.8 & 3.2 & 2.1 & 1.5 & 6.4 & 4.8 \\
\hline orf19.3538 & FRE9 & siderophore-iron transport & 4.7 & 14.9 & 2.5 & 3.6 & 12.6 & 4.3 \\
\hline orf19.4711 & & & 4.7 & 4.8 & 3.6 & 3.3 & 13.7 & 6.0 \\
\hline orf19.6840 & & & 4.7 & 3.0 & 12.1 & 18.1 & 34.7 & 10.0 \\
\hline orf19.4056 & GAT2 & filamentous growth & 4.6 & 5.1 & 2.7 & 3.9 & 2.9 & 0.6 \\
\hline orf19.7221 & SET3 & filamentous growth & 4.6 & 2.9 & 7.6 & 15.7 & 2.1 & 1.4 \\
\hline orf19.6970 & & & 4.5 & 4.4 & 2.3 & 2.4 & 2.0 & 1.8 \\
\hline orf19.7313 & SSU1 & filamentous growth & 4.5 & 4.8 & 1.3 & 1.1 & 6.8 & 4.0 \\
\hline orf19.5850 & NOC2 & filamentous growth & 4.5 & 4.8 & 2.1 & 3.5 & 8.7 & 9.5 \\
\hline orf19.6148 & & microtubule nucleation & 4.4 & 47.3 & 4.6 & 5.2 & 2.0 & 13.8 \\
\hline orf19.4899 & $G C A 1$ & biofilm formation & 4.4 & 5.2 & 3.7 & 3.2 & 1.2 & 1.9 \\
\hline orf19.5874 & & & 4.4 & 6.6 & 31.0 & 10.4 & 14.5 & 8.2 \\
\hline orf19.7503 & $C D A 2$ & ascospore wall assembly & 4.4 & 2.4 & 3.8 & 1.5 & 6.4 & 14.0 \\
\hline orf19.6306 & & carnitine biosynthetic process & 4.4 & 2.7 & 4.7 & 5.0 & 7.1 & 3.6 \\
\hline orf19.3369 & $\mathrm{MOH1}$ & & 4.3 & 8.1 & 6.4 & 5.9 & 18.6 & 13.3 \\
\hline orf19.3902 & & & 4.3 & 4.5 & 2.8 & 1.6 & 10.4 & 32.6 \\
\hline orf19.6888 & & filamentous growth & 4.3 & 5.7 & 1.4 & 1.1 & 8.8 & 11.4 \\
\hline orf19.2542 & & & 4.3 & 2.1 & 2.2 & 3.3 & 0.5 & 3.7 \\
\hline orf19.4546 & HOL4 & drug transport & 4.3 & 5.1 & 1.7 & 2.3 & 6.9 & 4.1 \\
\hline orf19.7500 & $P X A 1$ & fatty acid transport & 4.3 & 5.2 & 4.3 & 6.3 & 8.2 & 4.7 \\
\hline orf19.3121 & $G S T 1$ & regulation of nitrogen utilization & 4.2 & 2.7 & 5.8 & 19.0 & 1.5 & 7.8 \\
\hline
\end{tabular}


Table A-1. (Continued)

\begin{tabular}{|c|c|c|c|c|c|c|c|c|}
\hline \multirow[b]{3}{*}{$\begin{array}{c}\text { orf19 } \\
\text { Designation }\end{array}$} & \multirow[b]{3}{*}{$\begin{array}{c}\text { CGD } \\
\text { Name }\end{array}$} & \multirow[b]{3}{*}{ Process $^{a}$} & \multicolumn{6}{|c|}{ Change in Fold Expression } \\
\hline & & & \multicolumn{2}{|c|}{$\begin{array}{c}\text { SC5314+FLC } \\
\text { /SC5314 } \\
\end{array}$} & \multicolumn{2}{|c|}{$\begin{array}{c}\operatorname{cas} 5 \Delta / \Delta+\mathrm{FLC} \\
/ \operatorname{cas} 5 \Delta / \Delta \\
\end{array}$} & \multicolumn{2}{|c|}{$\begin{array}{c}u p c 2 \Delta / \Delta+\mathrm{FLC} / \\
u p c 2 \Delta / \Delta\end{array}$} \\
\hline & & & Exp1 & Exp2 & Exp1 & Exp2 & Exp1 & Exp2 \\
\hline orf19.1715 & IRO1 & cellular iron ion homeostasis & 4.2 & 8.1 & 2.7 & 2.3 & 2.2 & 1.4 \\
\hline orf19.4128 & & & 4.2 & 3.4 & 2.0 & 3.4 & 2.0 & 2.1 \\
\hline orf19.6169 & ATO1 & & 4.2 & 4.3 & 2.4 & 1.8 & 24.8 & 33.3 \\
\hline orf19.5125 & & & 4.2 & 4.5 & 3.5 & 2.8 & 10.6 & 9.1 \\
\hline orf19.6254 & ANT1 & ATP transport & 4.2 & 2.3 & 2.8 & 3.2 & 5.4 & 4.0 \\
\hline orf19.711 & & & 4.1 & 4.1 & 9.8 & 6.2 & 8.9 & 5.2 \\
\hline orf19.1350 & & cell redox homeostasis & 4.1 & 4.2 & 6.6 & 5.8 & 25.2 & 19.6 \\
\hline orf19.1800 & & protein targeting to vacuole & 4.1 & 3.8 & 2.1 & 1.8 & 6.4 & 4.0 \\
\hline orf19.499 & & translational readthrough & 4.1 & 4.2 & 11.2 & 14.9 & 24.8 & 5.4 \\
\hline orf19.1609 & & endonucleolytic cleavage of rRNA & 4.1 & 5.0 & 3.4 & 3.5 & 3.8 & 6.6 \\
\hline orf19.695 & $R G S 2$ & termination of G-protein coupled receptor signaling pathway & 4.1 & 5.8 & 3.3 & 3.4 & 5.8 & 5.2 \\
\hline orf19.4894 & & & 4.1 & 3.8 & 7.6 & 6.6 & 7.0 & 8.9 \\
\hline orf19.7457 & & & 4.1 & 2.4 & 4.6 & 4.4 & 11.2 & 12.7 \\
\hline orf19.76 & $S P B 1$ & maturation ofrRNA & 4.1 & 5.3 & 2.8 & 4.0 & 3.9 & 7.7 \\
\hline orf19.5735.3 & & & 4.0 & 3.1 & 4.1 & 3.4 & 2.9 & 2.5 \\
\hline orf19.7306 & & pyridoxal biosynthetic process & 4.0 & 3.9 & 5.1 & 5.9 & 11.6 & 15.6 \\
\hline orf19.2706 & CRH11 & cell wall organization & 4.0 & 3.3 & 4.1 & 2.7 & 3.9 & 2.3 \\
\hline orf19.3483 & & phosphatidylglycerol catabolic process & 4.0 & 2.4 & 2.0 & 2.0 & 5.1 & 8.4 \\
\hline orf19.7449 & & mitochondrial genome maintenance & 3.9 & 2.1 & 2.0 & 3.0 & 1.9 & 2.6 \\
\hline orf19.5759 & SNQ2 & filamentous growth & 3.9 & 6.9 & 5.4 & 8.0 & 4.2 & 2.0 \\
\hline orf19.5205 & & & 3.9 & 4.9 & 1.1 & 0.6 & 1.9 & 2.2 \\
\hline orf19.7043 & & & 3.9 & 2.8 & 0.9 & 1.0 & 2.4 & 2.1 \\
\hline orf19.1488 & & & 3.9 & 2.2 & 2.6 & 3.8 & 2.1 & 3.5 \\
\hline orf19.6554 & & calcium ion homeostasis & 3.9 & 3.8 & 6.8 & 7.5 & 7.6 & 3.9 \\
\hline orf19.2724 & & & 3.9 & 4.1 & 8.5 & 16.0 & 4.8 & 3.2 \\
\hline orf19.720 & GST3 & regulation of nitrogen utilization & 3.8 & 3.4 & 11.8 & 13.2 & 58.6 & 32.7 \\
\hline orf19.1915 & MPP10 & endonucleolytic cleavage of rRNA & 3.8 & 4.5 & 2.1 & 2.5 & 11.3 & 6.2 \\
\hline
\end{tabular}


Table A-1. (Continued)

\begin{tabular}{|c|c|c|c|c|c|c|c|c|}
\hline \multirow{3}{*}{$\begin{array}{c}\text { orf19 } \\
\text { Designation }\end{array}$} & \multirow{3}{*}{$\begin{array}{l}\text { CGD } \\
\text { Name }\end{array}$} & \multirow[b]{3}{*}{ Process $^{\mathbf{a}}$} & \multicolumn{6}{|c|}{ Change in Fold Expression } \\
\hline & & & \multicolumn{2}{|c|}{$\begin{array}{c}\text { SC5314+FLC } \\
\text { /SC5314 } \\
\end{array}$} & \multicolumn{2}{|c|}{$\begin{array}{c}\operatorname{cas} 5 \Delta / \Delta+\mathrm{FLC} \\
/ \operatorname{cas} 5 \Delta / \Delta \\
\end{array}$} & \multicolumn{2}{|c|}{$\begin{array}{c}u p c 2 \Delta / \Delta+\mathrm{FLC} / \\
u p c 2 \Delta / \Delta\end{array}$} \\
\hline & & & Exp1 & Exp2 & Exp1 & Exp2 & Exp1 & Exp2 \\
\hline orf19.6555 & & protein import into mitochondrial intermembrane space & 3.8 & 3.4 & 2.9 & 4.8 & 2.4 & 1.2 \\
\hline orf19.90 & & & 3.8 & 3.1 & 2.6 & 3.6 & 4.3 & 2.4 \\
\hline orf19.4873 & & & 3.8 & 13.5 & 14.6 & 7.1 & 57.4 & 24.8 \\
\hline orf19.1704 & FOX3 & fatty acid beta-oxidation & 3.8 & 2.7 & 5.0 & 7.0 & 6.9 & 4.3 \\
\hline orf19.7260 & & oxidation-reduction process & 3.8 & 3.5 & 2.8 & 3.3 & 2.3 & 2.8 \\
\hline orf19.6000 & $C D R 1$ & multidrug transport & 3.8 & 6.1 & 2.6 & 4.7 & 6.8 & 3.3 \\
\hline orf19.286 & & autophagy & 3.8 & 2.2 & 1.4 & 1.8 & 2.7 & 3.7 \\
\hline orf19.4779 & & drug transport & 3.8 & 4.7 & 2.8 & 3.4 & 9.7 & 8.8 \\
\hline orf19.3829 & PHR1 & cell wall organization & 3.8 & 5.0 & 2.8 & 3.8 & 14.9 & 7.0 \\
\hline orf19.3159 & UTP20 & endonucleolytic cleavage of rRNA & 3.8 & 8.9 & 1.9 & 3.5 & 5.4 & 2.7 \\
\hline orf19.5585 & SAP5 & pathogenesis & 3.7 & 3.0 & 0.9 & 1.0 & 1.4 & 2.3 \\
\hline orf19.3077 & VID21 & histone acetylation & 3.7 & 4.3 & 2.7 & 3.4 & 3.8 & 2.8 \\
\hline orf19.7156 & FAA2-3 & lipid metabolic process & 3.7 & 2.8 & 1.7 & 2.2 & 14.4 & 8.4 \\
\hline orf19.5565 & & valine metabolic process & 3.7 & 2.2 & 9.7 & 5.5 & 7.0 & 11.5 \\
\hline orf19.7384 & NOG1 & ribosomal large subunit biogenesis & 3.7 & 7.2 & 1.2 & 2.1 & 13.9 & 5.4 \\
\hline orf19.5454 & $D A L 1$ & allantoin assimilation pathway & 3.7 & 6.6 & 1.7 & 1.9 & 11.1 & 3.9 \\
\hline orf19.7635 & DRS1 & response to drug & 3.7 & 5.2 & 2.0 & 5.3 & 16.5 & 6.7 \\
\hline orf19.6838 & & fatty acid catabolic process & 3.7 & 2.1 & 2.5 & 3.2 & 6.2 & 7.5 \\
\hline orf19.3418 & & arginine catabolic process to ornithine & 3.7 & 2.2 & 3.0 & 2.3 & 11.4 & 7.6 \\
\hline orf19.7053 & $G A C 1$ & meiosis & 3.7 & 5.8 & 4.6 & 4.5 & 10.4 & 2.1 \\
\hline orf19.5248 & MSO1 & ascospore-type prospore membrane assembly & 3.7 & 2.7 & 2.8 & 3.8 & 3.5 & 3.2 \\
\hline orf19.4445 & & filamentous growth & 3.7 & 4.1 & 2.6 & 3.3 & 39.0 & 56.8 \\
\hline orf19.7422 & & rRNA modification & 3.7 & 3.9 & 2.0 & 2.5 & 4.1 & 5.4 \\
\hline orf19.1944 & GPR1 & filamentous growth & 3.7 & 4.6 & 2.5 & 5.5 & 10.5 & 3.2 \\
\hline orf19.1562 & & & 3.7 & 4.5 & 3.9 & 4.6 & 1.7 & 1.3 \\
\hline orf19.4743 & $A F G 1$ & & 3.7 & 4.6 & 2.8 & 2.5 & 9.3 & 5.9 \\
\hline orf19.3435 & & & 3.6 & 4.2 & 4.3 & 6.0 & 8.1 & 3.4 \\
\hline
\end{tabular}


Table A-1. (Continued)

\begin{tabular}{|c|c|c|c|c|c|c|c|c|}
\hline \multirow[b]{3}{*}{$\begin{array}{c}\text { orf19 } \\
\text { Designation }\end{array}$} & \multirow[b]{3}{*}{$\begin{array}{c}\text { CGD } \\
\text { Name }\end{array}$} & \multirow[b]{3}{*}{ Process $^{\mathrm{a}}$} & \multicolumn{6}{|c|}{ Change in Fold Expression } \\
\hline & & & \multicolumn{2}{|c|}{$\begin{array}{c}\text { SC5314+FLC } \\
\text { /SC5314 } \\
\end{array}$} & \multicolumn{2}{|c|}{$\begin{array}{c}\operatorname{cas} 5 \Delta / \Delta+F L C \\
\quad / c a s 5 \Delta / \Delta \\
\end{array}$} & \multicolumn{2}{|c|}{$\begin{array}{c}\text { upc } 2 \Delta / \Delta+\mathrm{FLC} / \\
u p c 2 \Delta / \Delta\end{array}$} \\
\hline & & & Exp1 & Exp2 & Exp1 & Exp2 & Exp1 & Exp2 \\
\hline orf19.1352 & TIM22 & protein import into mitochondrial inner membrane & 3.6 & 4.4 & 0.9 & 1.1 & 1.3 & 2.0 \\
\hline orf19.1523 & FMO1 & cellular response to drug & 3.6 & 5.6 & 2.5 & 4.3 & 4.3 & 5.7 \\
\hline orf19.6606 & & & 3.6 & 2.7 & 3.4 & 10.0 & 2.6 & 2.9 \\
\hline orf19.2859 & SRP40 & nucleocytoplasmic transport & 3.6 & 2.9 & 3.3 & 4.9 & 1.3 & 4.3 \\
\hline orf19.1728 & & & 3.6 & 2.3 & 43.7 & 33.3 & 15.7 & 5.0 \\
\hline orf19.4450.1 & & & 3.6 & 4.5 & 1.0 & 0.7 & 2.4 & 10.6 \\
\hline orf19.7042 & & & 3.6 & 2.3 & 9.4 & 7.6 & 5.8 & 4.7 \\
\hline orf19.5626 & & & 3.6 & 4.0 & 1.5 & 1.4 & 3.6 & 2.3 \\
\hline orf19.2492 & STE3 & cytogamy & 3.6 & 3.4 & 2.7 & 3.8 & 3.3 & 1.6 \\
\hline orf19.3710 & YHB5 & response to stress & 3.5 & 4.0 & 12.2 & 12.4 & 8.2 & 4.5 \\
\hline orf19.1727 & $P M C 1$ & cellular calcium ion homeostasis & 3.5 & 3.6 & 5.0 & 7.9 & 8.3 & 3.0 \\
\hline orf19.5019 & & & 3.5 & 3.7 & 1.7 & 2.1 & 0.9 & 1.9 \\
\hline orf19.7300 & & & 3.5 & 4.8 & 4.4 & 3.5 & 9.7 & 16.9 \\
\hline orf19.2400 & & nuclear mRNA splicing, via spliceosome & 3.5 & 1.5 & 0.9 & 1.2 & 1.5 & 2.3 \\
\hline orf19.5962 & SNF3 & carbohydrate transport & 3.5 & 3.0 & 4.7 & 7.1 & 5.9 & 2.1 \\
\hline orf19.4596 & & & 3.5 & 4.9 & 1.0 & 1.1 & 5.6 & 5.2 \\
\hline orf19.4505 & $A D H 3$ & fermentation & 3.5 & 1.6 & 5.8 & 2.4 & 2.0 & 1.4 \\
\hline orf19.4450 & $Z C F 23$ & regulation of transcription, DNA-dependent & 3.5 & 3.3 & 3.5 & 8.7 & 22.9 & 2.3 \\
\hline orf19.3261 & ATO9 & & 3.4 & 3.7 & 1.1 & 2.8 & 2.5 & 2.9 \\
\hline orf19.2836 & & carbon utilization & 3.4 & 5.9 & 3.7 & 4.3 & 1.6 & 1.5 \\
\hline orf19.661 & KRRl & endonucleolytic cleavage of rRNA & 3.4 & 3.0 & 3.2 & 4.6 & 4.0 & 4.8 \\
\hline orf19.7424 & $N S A 2$ & maturation of rRNA) & 3.4 & 2.8 & 2.1 & 3.1 & 2.6 & 4.0 \\
\hline orf19.6109 & TUP1 & filamentous growth & 3.4 & 3.2 & 3.1 & 3.1 & 4.2 & 2.1 \\
\hline orf19.2504 & BMS1 & ribosome assembly & 3.4 & 4.1 & 2.1 & 3.2 & 2.8 & 3.2 \\
\hline orf19.3646 & CTR1 & high-affinity iron ion transport & 3.4 & 9.1 & 3.1 & 4.9 & 10.9 & 10.4 \\
\hline orf19.3606 & & & 3.4 & 3.2 & 2.1 & 3.7 & 2.8 & 2.3 \\
\hline orf19.2832 & INN1 & chromosome organization & 3.4 & 3.9 & 2.9 & 3.7 & 10.3 & 10.2 \\
\hline
\end{tabular}


Table A-1. (Continued)

\begin{tabular}{|c|c|c|c|c|c|c|c|c|}
\hline \multirow[b]{3}{*}{$\begin{array}{c}\text { orf19 } \\
\text { Designation }\end{array}$} & \multirow[b]{3}{*}{$\begin{array}{l}\text { CGD } \\
\text { Name }\end{array}$} & \multirow[b]{3}{*}{ Process $^{\mathrm{a}}$} & \multicolumn{6}{|c|}{ Change in Fold Expression } \\
\hline & & & \multicolumn{2}{|c|}{$\begin{array}{c}\text { SC5314+FLC } \\
\text { /SC5314 } \\
\end{array}$} & \multicolumn{2}{|c|}{$\begin{array}{c}\operatorname{cas} 5 \Delta / \Delta+F L C \\
\quad / \operatorname{cas} 5 \Delta / \Delta \\
\end{array}$} & \multicolumn{2}{|c|}{$\begin{array}{c}\text { upc } 2 \Delta / \Delta+\mathrm{FLC} / \\
u p c 2 \Delta / \Delta\end{array}$} \\
\hline & & & Exp1 & Exp2 & Exp1 & $\operatorname{Exp2}$ & Exp1 & Exp2 \\
\hline orf19.7298 & $C H S 2$ & cell wall chitin biosynthetic process & 3.4 & 4.0 & 4.9 & 7.1 & 10.5 & 3.5 \\
\hline orf19.6589 & & regulation of cell size & 3.4 & 4.5 & 3.8 & 6.6 & 1.6 & 3.7 \\
\hline orf19.1646 & & endonucleolytic cleavage of rRNA & 3.4 & 3.1 & 1.8 & 2.8 & 2.8 & 5.4 \\
\hline orf19.1424 & & oxalate transport & 3.4 & 4.7 & 2.5 & 3.2 & 4.7 & 2.5 \\
\hline orf19.3815 & & ubiquitin-dependent protein catabolic process & 3.3 & 4.2 & 3.2 & 4.3 & 9.2 & 6.2 \\
\hline orf19.1481 & HAP42 & regulation of transcription, DNA-dependent & 3.3 & 4.1 & 3.8 & 6.0 & 2.7 & 2.0 \\
\hline orf19.5604 & $M D R 1$ & multidrug transport & 3.3 & 1.7 & 9.7 & 4.0 & 4.4 & 1.1 \\
\hline orf19.6202 & RBT4 & pathogenesis & 3.3 & 6.2 & 1.6 & 2.2 & 5.7 & 2.2 \\
\hline orf19.6725 & FUM12 & fumarate metabolic process & 3.3 & 6.4 & 3.8 & 6.4 & 5.7 & 2.5 \\
\hline orf19.2067 & & intracellular sequestering of iron ion & 3.3 & 3.3 & 2.4 & 2.8 & 4.0 & 4.8 \\
\hline orf19.4077 & MIT1 & sphingolipid biosynthetic process & 3.3 & 5.0 & 3.1 & 5.2 & 5.7 & 2.3 \\
\hline orf19.431 & $Z C F 2$ & regulation of transcription, DNA-dependent & 3.3 & 4.1 & 2.7 & 3.7 & 4.8 & 2.2 \\
\hline orf19.246 & & & 3.3 & 3.3 & 5.6 & 6.6 & 23.7 & 8.2 \\
\hline orf19.6313.2 & MVB12 & cellular response to alkalinity & 3.3 & 1.5 & 3.7 & 4.6 & 0.5 & 1.6 \\
\hline orf19.5118 & $S D S 24$ & cell separation during cytokinesis & 3.3 & 6.6 & 3.0 & 2.5 & 7.3 & 2.4 \\
\hline orf19.1449 & & & 3.3 & 2.6 & 5.5 & 8.4 & 15.9 & 39.3 \\
\hline orf19.5879 & & phosphatidic acid biosynthetic process & 3.3 & 2.9 & 1.5 & 1.2 & 3.3 & 2.8 \\
\hline orf19.5751 & ORM1 & response to drug & 3.3 & 3.6 & 1.6 & 1.7 & 3.4 & 1.8 \\
\hline orf19.2568 & IFU5 & & 3.3 & 3.4 & 4.6 & 4.0 & 6.0 & 2.9 \\
\hline orf19.192 & & & 3.3 & 3.8 & 3.7 & 3.7 & 12.2 & 3.9 \\
\hline orf19.5113 & $A D H 2$ & NADH oxidation & 3.3 & 9.7 & 11.4 & 16.0 & 23.0 & 10.1 \\
\hline orf19.5862 & AFP99 & & 3.3 & 2.0 & 2.1 & 2.7 & 13.0 & 10.1 \\
\hline orf19.5345 & & ER-associated protein catabolic process & 3.2 & 3.5 & 2.6 & 2.7 & 4.9 & 3.8 \\
\hline orf19.6648 & $S D A 1$ & actin cytoskeleton organization & 3.2 & 6.8 & 1.6 & 3.2 & 6.5 & 8.8 \\
\hline orf19.962 & & & 3.2 & 2.8 & 2.1 & 3.6 & 2.2 & 7.3 \\
\hline orf19.5010 & DIM1 & rRNA modification & 3.2 & 2.3 & 2.5 & 6.8 & 3.6 & 4.7 \\
\hline orf19.6236 & & ribosomal small subunit biogenesis & 3.2 & 3.2 & 2.7 & 5.0 & 3.1 & 5.7 \\
\hline
\end{tabular}


Table A-1. (Continued)

\begin{tabular}{|c|c|c|c|c|c|c|c|c|}
\hline \multirow[b]{3}{*}{$\begin{array}{c}\text { orf19 } \\
\text { Designation }\end{array}$} & \multirow[b]{3}{*}{$\begin{array}{l}\text { CGD } \\
\text { Name } \\
\end{array}$} & \multirow[b]{3}{*}{ Process $^{\mathrm{a}}$} & \multicolumn{6}{|c|}{ Change in Fold Expression } \\
\hline & & & \multicolumn{2}{|c|}{$\begin{array}{c}\text { SC5314+FLC } \\
\text { /SC5314 } \\
\end{array}$} & \multicolumn{2}{|c|}{$\begin{array}{c}\operatorname{cas} 5 \Delta / \Delta+\mathrm{FLC} \\
/ \operatorname{cas} 5 \Delta / \Delta\end{array}$} & \multicolumn{2}{|c|}{$\begin{array}{c}u p c 2 \Delta / \Delta+\mathrm{FLC} / \\
u p c 2 \Delta / \Delta\end{array}$} \\
\hline & & & Exp1 & Exp2 & Exp1 & Exp2 & Exp1 & Exp2 \\
\hline orf19.1578 & & endonucleolytic cleavage of rRNA & 3.2 & 3.2 & 1.3 & 2.0 & 2.4 & 2.2 \\
\hline orf19.7469 & $A R G 1$ & arginine biosynthetic process & 3.2 & 2.8 & 11.5 & 17.1 & 7.6 & 5.7 \\
\hline orf19.4391 & & & 3.2 & 1.5 & 3.9 & 7.0 & 1.0 & 0.7 \\
\hline orf19.2831 & RPC31 & tRNA transcription from RNA polymerase III promoter & 3.2 & 13.3 & 2.9 & 2.4 & 2.4 & 3.9 \\
\hline orf19.4883 & & signal transduction & 3.2 & 3.5 & 4.4 & 4.6 & 22.5 & 12.4 \\
\hline orf19.7219 & FTR1 & high-affinity iron ion transport & 3.2 & 7.8 & 0.5 & 1.1 & 0.1 & 0.0 \\
\hline orf19.4492 & & nuclear division & 3.2 & 3.6 & 2.3 & 3.7 & 1.5 & 3.1 \\
\hline orf19.2712 & HCA4 & rRNA processing & 3.2 & 3.3 & 4.1 & 3.7 & 3.2 & 5.1 \\
\hline orf19.4031 & & carbohydrate metabolic process & 3.2 & 4.0 & 2.0 & 2.9 & 2.9 & 1.7 \\
\hline orf19.4979 & KNS1 & protein amino acid phosphorylation & 3.2 & 3.3 & 2.2 & 3.2 & 22.9 & 19.0 \\
\hline orf19.857 & FMO2 & oxidation-reduction process & 3.1 & 2.6 & 3.5 & 4.9 & 4.8 & 3.2 \\
\hline orf19.4015 & $C A G 1$ & cellular morphogenesis during conjugation with cellular fusion & 3.1 & 3.6 & 0.8 & 0.7 & 4.4 & 3.3 \\
\hline orf19.3984 & & & 3.1 & 6.2 & 5.6 & 6.0 & 8.0 & 5.6 \\
\hline orf19.1642 & & endonucleolytic cleavage of rRNA & 3.1 & 2.6 & 6.4 & 3.4 & 3.3 & 5.9 \\
\hline orf19.2785 & ATP7 & ATP synthesis coupled proton transport & 3.1 & 3.1 & 2.0 & 2.6 & 1.3 & 1.5 \\
\hline orf19.4593 & $R G A 2$ & hyphal growth & 3.1 & 4.2 & 2.9 & 4.9 & 5.7 & 3.3 \\
\hline orf19.2414 & & & 3.1 & 3.3 & 1.1 & 0.6 & 0.7 & 1.7 \\
\hline orf19.414 & & translation & 3.1 & 3.0 & 4.9 & 12.3 & 1.6 & 4.8 \\
\hline orf19.6678 & & & 3.1 & 5.1 & 1.2 & 1.4 & 3.4 & 3.1 \\
\hline orf19.296 & & & 3.1 & 1.7 & 1.5 & 0.9 & 1.2 & 1.0 \\
\hline orf19.2943 & & amino acid transport & 3.1 & 4.6 & 2.3 & 3.4 & 6.4 & 2.3 \\
\hline orf19.3910 & & & 3.1 & 2.6 & 1.1 & 0.9 & 7.6 & 5.3 \\
\hline orf19.1719 & $S G A 1$ & glycogen catabolic process & 3.1 & 2.5 & 3.7 & 3.3 & 4.0 & 1.5 \\
\hline orf19.3813 & & & 3.1 & 3.1 & 1.4 & 1.8 & 6.3 & 3.4 \\
\hline orf19.6248 & & & 3.1 & 12.1 & 0.7 & 1.2 & 25.0 & 3.1 \\
\hline orf19.113 & $C I P 1$ & response to oxidative stress & 3.0 & 3.7 & 10.2 & 5.3 & 11.2 & 6.5 \\
\hline orf19.6126 & $K G D 2$ & 2-oxoglutarate metabolic process & 3.0 & 4.0 & 3.5 & 5.1 & 4.3 & 2.9 \\
\hline
\end{tabular}


Table A-1. (Continued)

\begin{tabular}{|c|c|c|c|c|c|c|c|c|}
\hline \multirow[b]{3}{*}{$\begin{array}{c}\text { orf19 } \\
\text { Designation }\end{array}$} & \multirow[b]{3}{*}{$\begin{array}{c}\text { CGD } \\
\text { Name }\end{array}$} & \multirow[b]{3}{*}{ Process $^{a}$} & \multicolumn{6}{|c|}{ Change in Fold Expression } \\
\hline & & & \multicolumn{2}{|c|}{$\begin{array}{c}\text { SC5314+FLC } \\
\text { /SC5314 } \\
\end{array}$} & \multicolumn{2}{|c|}{$\begin{array}{c}\operatorname{cas} 5 \Delta / \Delta+\mathrm{FLC} \\
/ \operatorname{cas} 5 \Delta / \Delta \\
\end{array}$} & \multicolumn{2}{|c|}{$\begin{array}{c}\text { upc } 2 \Delta / \Delta+\mathrm{FLC} / \\
u p c 2 \Delta / \Delta\end{array}$} \\
\hline & & & Exp1 & Exp2 & Exp1 & Exp2 & Exp1 & Exp2 \\
\hline orf19.5799 & & & 3.0 & 2.5 & 13.8 & 11.3 & 6.7 & 1.8 \\
\hline orf19.6348 & & ubiquitin-dependent protein catabolic process & 3.0 & 2.4 & 1.3 & 1.4 & 0.6 & 1.2 \\
\hline orf19.4191 & $R L P 24$ & ribosomal large subunit biogenesis & 3.0 & 3.6 & 1.9 & 3.0 & 1.2 & 4.9 \\
\hline orf19.288 & MET13 & methionine metabolic process & 3.0 & 4.3 & 3.0 & 4.2 & 4.0 & 1.9 \\
\hline orf19.7380 & & & 3.0 & 3.2 & 1.8 & 2.5 & 1.6 & 1.3 \\
\hline orf19.3707 & $Y H B 1$ & nitric oxide catabolic process & 3.0 & 2.8 & 0.9 & 0.6 & 1.3 & 2.0 \\
\hline orf19.1980 & GIT4 & phospholipid transport & 3.0 & 2.1 & 1.5 & 1.8 & 3.4 & 3.1 \\
\hline orf19.24 & $R T A 2$ & lipid translocation & 3.0 & 5.5 & 9.4 & 11.1 & 2.0 & 1.1 \\
\hline orf19.872 & & & 3.0 & 2.0 & 2.1 & 4.1 & 3.6 & 2.4 \\
\hline orf19.3540 & $M A K 5$ & rRNA processing & 3.0 & 2.9 & 1.9 & 3.4 & 3.5 & 4.1 \\
\hline orf19.515 & & & 3.0 & 2.2 & 2.9 & 5.3 & 4.8 & 16.5 \\
\hline orf19.3544 & & oxidation-reduction process & 3.0 & 3.2 & 2.5 & 2.6 & 7.2 & 4.7 \\
\hline orf19.4183 & MUC1 & & 3.0 & 3.5 & 4.8 & 3.6 & 9.9 & 3.1 \\
\hline orf19.3762 & & & 2.9 & 1.8 & 1.4 & 1.3 & 1.6 & 4.5 \\
\hline orf19.2905 & & & 2.9 & 3.2 & 2.9 & 2.3 & 11.9 & 14.5 \\
\hline orf19.2386 & & endonucleolytic cleavage of rRNA & 2.9 & 1.7 & 3.0 & 4.9 & 2.9 & 6.4 \\
\hline orf19.5169 & & & 2.9 & 2.2 & 9.5 & 9.7 & 2.9 & 2.0 \\
\hline orf19.4793 & & & 2.9 & 2.0 & 1.5 & 2.9 & 2.6 & 2.3 \\
\hline orf19.1369 & & metabolic process & 2.9 & 2.3 & 1.2 & 1.0 & 4.1 & 3.5 \\
\hline orf19.3661 & & protein deubiquitination & 2.9 & 3.0 & 2.4 & 2.3 & 4.1 & 1.9 \\
\hline orf19.5026 & & regulation of transcription, DNA-dependent & 2.9 & 3.2 & 5.1 & 10.3 & 7.0 & 2.6 \\
\hline orf19.2185 & NSA1 & ribosomal large subunit biogenesis & 2.9 & 2.6 & 1.8 & 3.1 & 1.1 & 4.0 \\
\hline orf19.7547 & & protein ubiquitination & 2.9 & 3.0 & 1.1 & 1.4 & 3.9 & 2.6 \\
\hline orf19.2167 & & ribosomal large subunit biogenesis & 2.9 & 1.8 & 2.9 & 4.8 & 1.6 & 3.2 \\
\hline orf19.1774 & & oxidation-reduction process & 2.9 & 2.7 & 3.2 & 6.0 & 17.3 & 2.3 \\
\hline orf19.6758 & & response to salt stress & 2.9 & 1.9 & 3.7 & 2.6 & 2.9 & 2.8 \\
\hline orf19.4510 & IFA4 & & 2.9 & 3.7 & 2.2 & 2.2 & 2.5 & 2.7 \\
\hline
\end{tabular}


Table A-1. (Continued)

\begin{tabular}{|c|c|c|c|c|c|c|c|c|}
\hline \multirow{3}{*}{$\begin{array}{c}\text { orf19 } \\
\text { Designation }\end{array}$} & \multirow{3}{*}{$\begin{array}{l}\text { CGD } \\
\text { Name }\end{array}$} & \multirow[b]{3}{*}{ Process $^{\mathrm{a}}$} & \multicolumn{6}{|c|}{ Change in Fold Expression } \\
\hline & & & \multicolumn{2}{|c|}{$\begin{array}{c}\text { SC5314+FLC } \\
\text { /SC5314 } \\
\end{array}$} & \multicolumn{2}{|c|}{$\begin{array}{c}\operatorname{cas} 5 \Delta / \Delta+\mathrm{FLC} \\
/ \operatorname{cas} 5 \Delta / \Delta\end{array}$} & \multicolumn{2}{|c|}{$\begin{array}{c}\text { upc } 2 \Delta / \Delta+\mathrm{FLC} / \\
u p c 2 \Delta / \Delta\end{array}$} \\
\hline & & & Exp1 & Exp2 & Exp1 & $\operatorname{Exp2}$ & Exp1 & Exp2 \\
\hline orf19.376 & & & 2.9 & 3.4 & 1.1 & 1.5 & 7.6 & 5.5 \\
\hline orf19.35 & & protein phosphorylation & 2.9 & 3.3 & 2.3 & 2.9 & 1.9 & 1.6 \\
\hline orf19.3736 & KAR4 & G1 phase of mitotic cell cycle & 2.8 & 2.6 & 1.2 & 1.0 & 2.6 & 1.5 \\
\hline orf19.2752 & $A D R 1$ & filamentous growth & 2.8 & 4.4 & 5.5 & 11.9 & 8.2 & 3.9 \\
\hline orf19.4043 & & response to stress & 2.8 & 4.5 & 1.6 & 1.7 & 5.4 & 3.0 \\
\hline orf19.3756 & CHR1 & nuclear mRNA splicing, via spliceosome & 2.8 & 3.1 & 3.2 & 3.5 & 2.0 & 3.7 \\
\hline orf19.5295 & & & 2.8 & 1.8 & 2.1 & 2.0 & 22.1 & 16.6 \\
\hline orf19.4657 & & regulation of lipid biosynthetic process & 2.8 & 2.2 & 3.5 & 6.1 & 1.4 & 0.6 \\
\hline orf19.7229 & $I M L 2$ & & 2.8 & 3.3 & 1.4 & 1.4 & 3.8 & 1.9 \\
\hline orf19.4479 & & endonucleolytic cleavage of rRNA & 2.8 & 2.7 & 1.7 & 3.8 & 2.5 & 4.0 \\
\hline orf19.4383 & & response to DNA damage stimulus & 2.8 & 2.6 & 0.9 & 0.8 & 1.4 & 2.2 \\
\hline orf19.3848 & & & 2.8 & 1.8 & 1.8 & 4.3 & 1.6 & 1.1 \\
\hline orf19.4211 & FET3 & high-affinity iron ion transport & 2.8 & 6.2 & 1.6 & 1.7 & 0.7 & 0.2 \\
\hline orf19.287 & & & 2.8 & 2.9 & 1.7 & 2.3 & 1.4 & 1.5 \\
\hline orf19.3240 & $E R G 27$ & ergosterol biosynthetic process & 2.8 & 2.4 & 2.3 & 2.8 & 1.4 & 1.1 \\
\hline orf19.2285 & & response to drug & 2.8 & 1.5 & 5.0 & 2.8 & 2.9 & 83.5 \\
\hline orf19.5547 & & & 2.8 & 2.4 & 2.0 & 1.8 & 0.8 & 1.1 \\
\hline orf19.7520 & POT1 & fatty acid beta-oxidation & 2.8 & 2.3 & 4.8 & 6.9 & 3.6 & 5.1 \\
\hline orf19.1388 & & ribosomal large subunit biogenesis & 2.8 & 2.1 & 2.7 & 5.2 & 1.3 & 6.9 \\
\hline orf 19.4255 & ECM331 & & 2.7 & 5.7 & 5.4 & 5.7 & 5.0 & 1.5 \\
\hline orf19.1411 & & oxidation-reduction process & 2.7 & 4.8 & 1.1 & 0.9 & 1.4 & 1.1 \\
\hline orf19.2154 & $H X K 1$ & hyphal growth & 2.7 & 2.8 & 1.4 & 2.2 & 3.1 & 1.6 \\
\hline orf19.3015 & $A R X 1$ & ribosomal large subunit biogenesis & 2.7 & 3.9 & 1.3 & 1.7 & 3.7 & 2.8 \\
\hline orf19.4887 & ECM21 & ubiquitin-dependent endocytosis & 2.7 & 3.1 & 0.8 & 1.0 & 4.0 & 5.0 \\
\hline orf19.3239 & CTF18 & double-strand break repair via homologous recombination & 2.7 & 2.4 & 1.0 & 1.1 & 1.4 & 0.8 \\
\hline orf19.4093 & PES1 & filamentous growth & 2.7 & 3.5 & 2.3 & 2.3 & 0.6 & 1.3 \\
\hline orf19.2344 & $A S R 1$ & & 2.7 & 1.7 & 10.9 & 7.9 & 7.1 & 2.5 \\
\hline
\end{tabular}


Table A-1. (Continued)

\begin{tabular}{|c|c|c|c|c|c|c|c|c|}
\hline \multirow{3}{*}{$\begin{array}{c}\text { orf19 } \\
\text { Designation }\end{array}$} & \multirow{3}{*}{$\begin{array}{c}\text { CGD } \\
\text { Name }\end{array}$} & \multirow[b]{3}{*}{ Process $^{\mathrm{a}}$} & \multicolumn{6}{|c|}{ Change in Fold Expression } \\
\hline & & & \multicolumn{2}{|c|}{$\begin{array}{c}\text { SC5314+FLC } \\
\text { /SC5314 } \\
\end{array}$} & \multicolumn{2}{|c|}{$\begin{array}{c}\operatorname{cas} 5 \Delta / \Delta+\mathrm{FLC} \\
/ \operatorname{cas} 5 \Delta / \Delta \\
\end{array}$} & \multicolumn{2}{|c|}{$\begin{array}{c}u p c 2 \Delta / \Delta+\mathrm{FLC} / \\
u p c 2 \Delta / \Delta\end{array}$} \\
\hline & & & Exp1 & Exp2 & Exp1 & Exp2 & Exp1 & Exp2 \\
\hline orf19.2458 & & cellular response to glucose starvation & 2.7 & 2.6 & 3.8 & 5.8 & 7.8 & 6.5 \\
\hline orf19.1714 & $P G A 44$ & & 2.7 & 2.3 & 1.6 & 0.8 & 201.7 & 19.2 \\
\hline orf19.183 & HIS3 & histidine biosynthetic process & 2.7 & 1.7 & 4.7 & 4.1 & 8.4 & 7.4 \\
\hline orf19.419 & & & 2.7 & 6.4 & 4.7 & 3.5 & 30.5 & 8.7 \\
\hline orf19.2319 & & rRNA processing & 2.7 & 2.3 & 2.5 & 3.3 & 1.5 & 2.0 \\
\hline orf19.5991 & & ribosomal large subunit assembly & 2.7 & 4.4 & 2.7 & 3.7 & 6.1 & 7.5 \\
\hline orf19.6674 & BTS1 & terpenoid biosynthetic process & 2.7 & 2.1 & 1.3 & 1.4 & 3.3 & 2.3 \\
\hline orf19.4346 & & COPII vesicle coating & 2.7 & 2.7 & 1.9 & 2.3 & 1.7 & 1.0 \\
\hline orf19.1809 & FOX2 & fatty acid beta-oxidation & 2.7 & 4.0 & 6.8 & 7.7 & 6.0 & 5.7 \\
\hline orf19.6608 & & & 2.7 & 4.7 & 1.5 & 1.3 & 5.8 & 9.0 \\
\hline orf19.2090 & & maturation of SSU-rRNA & 2.7 & 3.3 & 1.8 & 2.9 & 2.8 & 4.4 \\
\hline orf19.2768 & $A M S 1$ & oligosaccharide catabolic process & 2.7 & 3.9 & 2.1 & 1.5 & 2.9 & 2.7 \\
\hline orf19.5251 & ZCF30 & regulation of transcription from RNA polymerase II promoter & 2.7 & 3.1 & 3.0 & 4.0 & 4.6 & 3.1 \\
\hline orf19.4961 & STP2 & amino acid import & 2.7 & 3.2 & 1.6 & 2.4 & 2.8 & 1.9 \\
\hline orf19.4531 & & transport & 2.7 & 4.4 & 5.5 & 12.3 & 1.5 & 0.6 \\
\hline orf19.4204 & & & 2.6 & 2.2 & 4.1 & 4.5 & 0.8 & 1.6 \\
\hline orf19.6955 & $H B R 3$ & maturation of rRNA & 2.6 & 2.3 & 2.3 & 4.3 & 6.8 & 6.4 \\
\hline orf19.2710 & & chromosome segregation & 2.6 & 1.8 & 1.0 & 0.9 & 1.0 & 2.5 \\
\hline orf19.6941 & & lipid storage & 2.6 & 3.6 & 2.8 & 2.8 & 7.2 & 6.1 \\
\hline orf19.6659 & GAP6 & amino acid transport & 2.6 & 3.0 & 1.4 & 1.7 & 2.1 & 1.8 \\
\hline orf19.6830 & & metabolic process & 2.6 & 2.7 & 4.4 & 6.5 & 8.9 & 5.3 \\
\hline orf19.921 & HMS1 & filamentous growth & 2.6 & 2.8 & 2.0 & 2.6 & 0.8 & 1.3 \\
\hline orf19.517 & HAP31 & regulation of carbohydrate metabolic process & 2.6 & 1.5 & 2.6 & 4.8 & 2.9 & 3.8 \\
\hline orf19.4114 & FAA2-1 & lipid metabolic process & 2.6 & 2.4 & 3.5 & 3.5 & 6.9 & 6.4 \\
\hline orf19.6843 & & mitotic sister chromatid segregation & 2.6 & 3.0 & 2.7 & 3.1 & 9.2 & 10.1 \\
\hline orf19.1596 & FGR28 & filamentous growth & 2.6 & 2.9 & 1.3 & 1.8 & 1.8 & 1.9 \\
\hline orf19.4013 & & & 2.6 & 2.2 & 0.6 & 0.6 & 0.3 & 0.4 \\
\hline
\end{tabular}


Table A-1. (Continued)

\begin{tabular}{|c|c|c|c|c|c|c|c|c|}
\hline \multirow[b]{3}{*}{$\begin{array}{c}\text { orf19 } \\
\text { Designation }\end{array}$} & \multirow[b]{3}{*}{$\begin{array}{l}\text { CGD } \\
\text { Name }\end{array}$} & \multirow[b]{3}{*}{ Process $^{\mathrm{a}}$} & \multicolumn{6}{|c|}{ Change in Fold Expression } \\
\hline & & & \multicolumn{2}{|c|}{$\begin{array}{c}\text { SC5314+FLC } \\
\text { /SC5314 }\end{array}$} & \multicolumn{2}{|c|}{$\begin{array}{l}\operatorname{cas} 5 \Delta / \Delta+F L C \\
/ \operatorname{cas} 5 \Delta / \Delta\end{array}$} & \multicolumn{2}{|c|}{$\begin{array}{c}u p c 2 \Delta / \Delta+\mathrm{FLC} / \\
u p c 2 \Delta / \Delta\end{array}$} \\
\hline & & & Exp1 & Exp2 & Exp1 & Exp2 & Exp1 & Exp2 \\
\hline orf19.3512 & & cell wall organization & 2.6 & 2.8 & 0.9 & 1.2 & 8.0 & 6.6 \\
\hline orf19.3865 & $R F X 1$ & negative regulation of transcription from RNA pol II promoter & 2.6 & 2.6 & 6.5 & 5.9 & 21.5 & 9.7 \\
\hline orf19.7456 & & & 2.6 & 2.6 & 3.6 & 4.9 & 2.2 & 0.6 \\
\hline orf19.2942 & DIP5 & amino acid transport & 2.6 & 2.5 & 1.8 & 2.8 & 3.6 & 2.0 \\
\hline orf19.2432 & $H A C l$ & cell wall organization & 2.6 & 4.3 & 2.5 & 2.8 & 13.2 & 5.4 \\
\hline orf19.4731 & & intra-Golgi vesicle-mediated transport & 2.6 & 2.6 & 2.0 & 2.4 & 1.2 & 1.5 \\
\hline orf19.5299 & ECM1 & cell wall organization & 2.6 & 4.8 & 2.7 & 3.5 & 1.5 & 5.3 \\
\hline orf19.7398 & & ribosomal large subunit biogenesis & 2.6 & 2.4 & 2.4 & 3.9 & 3.0 & 3.8 \\
\hline orf19.3021 & & & 2.6 & 2.0 & 1.2 & 0.9 & 2.4 & 2.2 \\
\hline orf19.809 & & maturation of rRNA & 2.6 & 1.8 & 2.2 & 3.4 & 2.9 & 7.1 \\
\hline orf19.5710 & & NLS-bearing substrate import into nucleus & 2.6 & 3.2 & 4.0 & 2.3 & 3.5 & 2.2 \\
\hline orf19.5876 & & & 2.6 & 2.3 & 1.8 & 1.6 & 7.3 & 7.1 \\
\hline orf19.5459 & & mRNA polyadenylation & 2.6 & 2.3 & 4.2 & 4.3 & 3.9 & 2.3 \\
\hline orf19.3080 & & mitotic sister chromatid cohesion & 2.6 & 2.9 & 4.7 & 4.8 & 11.8 & 4.9 \\
\hline orf19.996 & & filamentous growth & 2.6 & 3.3 & 3.5 & 3.5 & 7.0 & 4.3 \\
\hline orf19.6143 & & oxidation-reduction process & 2.6 & 2.6 & 3.6 & 3.4 & 11.5 & 6.8 \\
\hline orf19.6999 & & oxidation-reduction process & 2.6 & 1.7 & 2.2 & 2.6 & 4.0 & 1.4 \\
\hline orf19.496 & & mismatch repair & 2.6 & 2.3 & 1.6 & 2.3 & 1.1 & 0.6 \\
\hline orf19.2059 & & & 2.6 & 2.3 & 0.2 & 0.3 & 2.3 & 17.6 \\
\hline orf19.1867 & & malate transport & 2.6 & 4.2 & 0.7 & 0.6 & 15.1 & 62.1 \\
\hline orf19.3737 & & & 2.5 & 2.4 & 4.6 & 5.7 & 8.0 & 4.1 \\
\hline orf19.1865 & & reciprocal meiotic recombination & 2.5 & 2.7 & 2.0 & 2.8 & 3.3 & 1.5 \\
\hline orf19.6605 & & & 2.5 & 2.1 & 3.8 & 4.1 & 5.7 & 4.4 \\
\hline orf19.281 & & & 2.5 & 2.2 & 1.5 & 1.9 & 3.8 & 3.2 \\
\hline orf19.5145 & SSP96 & oxidation-reduction process & 2.5 & 1.7 & 1.2 & 2.6 & 18.0 & 9.3 \\
\hline orf19.6322 & $A R D$ & fatty acid catabolic process & 2.5 & 2.8 & 2.7 & 2.9 & 3.7 & 4.7 \\
\hline orf19.5963 & & protein amino acid glycosylation & 2.5 & 2.7 & 2.4 & 4.4 & 2.1 & 1.0 \\
\hline
\end{tabular}


Table A-1. (Continued)

\begin{tabular}{|c|c|c|c|c|c|c|c|c|}
\hline \multirow[b]{3}{*}{$\begin{array}{c}\text { orf19 } \\
\text { Designation }\end{array}$} & \multirow[b]{3}{*}{$\begin{array}{l}\text { CGD } \\
\text { Name }\end{array}$} & \multirow[b]{3}{*}{ Process $^{a}$} & \multicolumn{6}{|c|}{$\begin{array}{l}\text { Change in Fold Expression } \\
\end{array}$} \\
\hline & & & \multicolumn{2}{|c|}{$\begin{array}{c}\text { SC5314+FLC } \\
\text { /SC5314 } \\
\end{array}$} & \multicolumn{2}{|c|}{$\begin{array}{c}\operatorname{cas} 5 \Delta / \Delta+\mathrm{FLC} \\
/ \operatorname{cas} 5 \Delta / \Delta\end{array}$} & \multicolumn{2}{|c|}{$\begin{array}{c}u p c 2 \Delta / \Delta+\mathrm{FLC} / \\
u p c 2 \Delta / \Delta\end{array}$} \\
\hline & & & Exp1 & Exp2 & Exp1 & Exp2 & Exp1 & Exp2 \\
\hline orf19.4507 & & & 2.5 & 2.3 & 1.7 & 1.3 & 2.5 & 2.3 \\
\hline orf19.2350 & & filamentous growth & 2.5 & 4.1 & 0.6 & 1.0 & 0.9 & 0.6 \\
\hline orf19.2978 & & & 2.5 & 1.7 & 4.5 & 5.9 & 1.1 & 1.3 \\
\hline orf19.2398 & & & 2.5 & 3.8 & 1.3 & 1.7 & 4.5 & 3.0 \\
\hline orf19.5023 & $D A L 7$ & anion transport & 2.5 & 4.2 & 3.9 & 4.0 & 6.2 & 5.5 \\
\hline orf19.4870 & $D B P 3$ & rRNA processing & 2.5 & 3.1 & 1.1 & 2.4 & 2.0 & 3.8 \\
\hline orf19.278 & & & 2.5 & 2.7 & 1.4 & 1.5 & 2.2 & 1.9 \\
\hline orf19.7267 & SAM35 & mitochondrion organization & 2.5 & 2.1 & 2.0 & 2.3 & 5.2 & 3.1 \\
\hline orf19.1625 & & & 2.5 & 2.6 & 2.4 & 3.2 & 1.6 & 2.0 \\
\hline orf19.2772 & HOS3 & histone deacetylation & 2.5 & 2.6 & 2.2 & 2.2 & 1.4 & 1.1 \\
\hline orf19.6102 & $R C A 1$ & DNA metabolic process & 2.5 & 3.4 & 1.2 & 1.2 & 1.1 & 1.4 \\
\hline orf19.4495 & NDH51 & biofilm formation & 2.5 & 3.3 & 2.4 & 3.4 & 2.7 & 1.6 \\
\hline orf19.3008 & COQ4 & ubiquinone biosynthetic process & 2.5 & 1.8 & 1.6 & 1.7 & 0.4 & 0.8 \\
\hline orf19.6997 & ATO5 & cellular response to alkalinity & 2.5 & 2.6 & 0.8 & 0.7 & 2.7 & 2.9 \\
\hline orf19.4264 & & & 2.5 & 3.2 & 1.2 & 1.0 & 0.8 & 3.2 \\
\hline orf19.7115 & $S A C 7$ & actin filament reorganization during cell cycle & 2.5 & 3.5 & 8.6 & 7.9 & 6.9 & 3.1 \\
\hline orf19.4590 & $R F X 2$ & adhesion to host & 2.5 & 4.5 & 1.5 & 3.3 & 23.4 & 8.1 \\
\hline orf19.93 & & development of symbiont in host & 2.5 & 2.4 & 2.4 & 2.8 & 1.8 & 2.5 \\
\hline orf19.6057 & & pantothenate biosynthetic process & 2.5 & 1.8 & 0.9 & 1.0 & 0.2 & 0.2 \\
\hline orf19.4477 & CSH1 & biofilm formation & 2.5 & 2.3 & 2.2 & 2.1 & 3.9 & 2.3 \\
\hline orf19.5938 & SEN1 & rRNA processing & 2.5 & 5.2 & 1.5 & 2.5 & 8.0 & 1.9 \\
\hline orf19.2066.1 & ATP18 & ATP synthesis coupled proton transport & 2.5 & 2.3 & 2.0 & 2.6 & 2.5 & 2.8 \\
\hline orf19.6709 & & & 2.5 & 2.6 & 3.1 & 2.8 & 7.3 & 4.8 \\
\hline orf19.7650 & LTV1 & response to osmotic stress & 2.5 & 2.9 & 1.7 & 2.4 & 1.8 & 3.1 \\
\hline orf19.203 & STB3 & positive regulation of transcription from RNA polymerase II & 2.5 & 3.6 & 3.5 & 2.9 & 7.0 & 3.1 \\
\hline orf19.2838 & & response to drug & 2.5 & 2.9 & 0.8 & 0.7 & 1.6 & 0.9 \\
\hline orf19.7154 & UTP18 & endonucleolytic cleavage of rRNA & 2.4 & 1.5 & 1.6 & 3.1 & 2.1 & 2.9 \\
\hline
\end{tabular}


Table A-1. (Continued)

\begin{tabular}{|c|c|c|c|c|c|c|c|c|}
\hline \multirow{3}{*}{$\begin{array}{c}\text { orf19 } \\
\text { Designation }\end{array}$} & \multirow{3}{*}{$\begin{array}{l}\text { CGD } \\
\text { Name }\end{array}$} & \multirow[b]{3}{*}{ Process $^{\mathrm{a}}$} & \multicolumn{6}{|c|}{ Change in Fold Expression } \\
\hline & & & \multicolumn{2}{|c|}{$\begin{array}{c}\text { SC5314+FLC } \\
\text { /SC5314 } \\
\end{array}$} & \multicolumn{2}{|c|}{$\begin{array}{c}\operatorname{cas} 5 \Delta / \Delta+F L C \\
\quad / \operatorname{cas} 5 \Delta / \Delta \\
\end{array}$} & \multicolumn{2}{|c|}{$\begin{array}{c}u p c 2 \Delta / \Delta+\mathrm{FLC} / \\
u p c 2 \Delta / \Delta\end{array}$} \\
\hline & & & Exp1 & Exp2 & Exp1 & Exp2 & Exp1 & $\operatorname{Exp2}$ \\
\hline orf19.6003 & & intracellular signal transduction & 2.4 & 2.1 & 1.9 & 2.5 & 0.9 & 0.7 \\
\hline orf19.2909 & $E R G 26$ & ergosterol biosynthetic process & 2.4 & 1.7 & 0.9 & 0.9 & 0.7 & 1.0 \\
\hline orf19.3734 & $G E F 2$ & transmembrane transport & 2.4 & 4.0 & 1.5 & 1.6 & 10.1 & 4.1 \\
\hline orf19.3867 & RPL7 & exonucleolytic trimming of rRNA & 2.4 & 2.0 & 1.3 & 2.9 & 1.2 & 2.7 \\
\hline orf19.5588 & $P G A 60$ & & 2.4 & 3.3 & 1.3 & 1.9 & 3.1 & 1.3 \\
\hline orf19.2887 & & & 2.4 & 2.7 & 3.6 & 4.2 & 5.8 & 2.2 \\
\hline orf19.2954 & & & 2.4 & 2.9 & 1.5 & 2.1 & 1.8 & 1.7 \\
\hline orf19.6062.3 & & cristae formation & 2.4 & 2.9 & 2.7 & 2.9 & 3.2 & 2.5 \\
\hline orf19.7610 & PTP3 & osmosensory signaling pathway & 2.4 & 2.5 & 3.3 & 3.3 & 2.7 & 1.4 \\
\hline orf19.2707.1 & QCR9 & iron-sulfur cluster assembly & 2.4 & 2.1 & 1.3 & 4.5 & 0.7 & 1.3 \\
\hline orf19.2190 & $V R P 1$ & actin cortical patch localization & 2.4 & 2.4 & 3.5 & 5.1 & 3.1 & 2.0 \\
\hline orf19.2623 & ECM22 & sterol biosynthetic processs & 2.4 & 4.0 & 1.8 & 2.3 & 4.1 & 1.9 \\
\hline orf19.3298 & $\mathrm{CCH} 1$ & calcium ion transport & 2.4 & 3.5 & 2.0 & 1.6 & 7.6 & 3.2 \\
\hline orf19.5752 & & ubiquitin-dependent protein catabolic process & 2.4 & 1.8 & 1.5 & 1.4 & 2.7 & 2.5 \\
\hline orf19.6790 & & mRNA splice site selection & 2.4 & 3.0 & 3.5 & 5.5 & 7.1 & 1.3 \\
\hline orf19.5591 & $A D O 1$ & purine base metabolic process & 2.4 & 2.9 & 1.1 & 1.5 & 0.7 & 0.8 \\
\hline orf19.6905 & & & 2.4 & 3.3 & 0.5 & 0.4 & 1.2 & 1.4 \\
\hline orf19.804 & & transport & 2.4 & 1.6 & 1.6 & 2.1 & 2.3 & 3.4 \\
\hline orf19.4522 & & response to drug & 2.4 & 1.8 & 1.7 & 2.3 & 3.2 & 3.7 \\
\hline orf19.7680 & CTA26 & positive regulation of transcription, DNA-dependent & 2.4 & 2.4 & 3.0 & 4.3 & 3.2 & 8.3 \\
\hline orf19.2459 & & & 2.4 & 3.0 & 1.9 & 2.5 & 2.9 & 2.2 \\
\hline orf19.4191.1 & & protein polyubiquitination & 2.4 & 2.0 & 2.2 & 1.6 & 2.0 & 1.9 \\
\hline orf19.5368 & & ER-associated protein catabolic process & 2.4 & 1.8 & 3.6 & 4.3 & 4.1 & 2.0 \\
\hline orf19.1949 & VPS1 & biofilm formation & 2.4 & 2.6 & 3.4 & 2.8 & 5.6 & 2.4 \\
\hline orf19.3705 & PTC6 & protein amino acid dephosphorylation & 2.4 & 2.5 & 1.7 & 1.9 & 2.1 & 1.7 \\
\hline orf19.3109 & & & 2.4 & 2.9 & 0.9 & 1.6 & 7.8 & 3.2 \\
\hline orf19.6043 & & aerobic respiration & 2.4 & 2.2 & 3.1 & 2.8 & 4.7 & 2.6 \\
\hline
\end{tabular}


Table A-1. (Continued)

\begin{tabular}{|c|c|c|c|c|c|c|c|c|}
\hline \multirow[b]{3}{*}{$\begin{array}{c}\text { orf19 } \\
\text { Designation }\end{array}$} & \multirow[b]{3}{*}{$\begin{array}{c}\text { CGD } \\
\text { Name }\end{array}$} & \multirow[b]{3}{*}{ Process $^{a}$} & \multicolumn{6}{|c|}{ Change in Fold Expression } \\
\hline & & & \multicolumn{2}{|c|}{$\begin{array}{c}\text { SC5314+FLC } \\
\text { /SC5314 } \\
\end{array}$} & \multicolumn{2}{|c|}{$\begin{array}{c}\operatorname{cas} 5 \Delta / \Delta+\mathrm{FLC} \\
/ \operatorname{cas} 5 \Delta / \Delta \\
\end{array}$} & \multicolumn{2}{|c|}{$\begin{array}{c}u p c 2 \Delta / \Delta+\mathrm{FLC} / \\
u p c 2 \Delta / \Delta\end{array}$} \\
\hline & & & Exp1 & Exp2 & Exp1 & Exp2 & Exp1 & Exp2 \\
\hline orf19.7281 & $P D K 2$ & response to drug & 2.4 & 2.0 & 4.3 & 3.9 & 1.9 & 1.6 \\
\hline orf19.3276 & $P W P 2$ & cytokinesis & 2.4 & 3.8 & 1.4 & 2.4 & 3.7 & 2.6 \\
\hline orf19.6514 & CUP9 & cellular copper ion homeostasis & 2.4 & 2.3 & 2.4 & 3.1 & 4.9 & 2.2 \\
\hline orf19.3974 & PUT2 & glutamate biosynthetic process & 2.4 & 3.4 & 3.0 & 3.3 & 3.1 & 1.2 \\
\hline orf19.6638 & PTC4 & protein dephosphorylation & 2.4 & 2.1 & 1.2 & 2.1 & 0.9 & 1.2 \\
\hline orf19.6690 & & & 2.4 & 2.3 & 0.7 & 0.6 & 0.6 & 0.7 \\
\hline orf19.6417 & TSR1 & rRNA processing & 2.4 & 3.6 & 1.5 & 3.5 & 5.2 & 3.1 \\
\hline orf19.4897 & & phospholipid transport & 2.4 & 2.4 & 1.3 & 1.2 & 1.0 & 1.4 \\
\hline orf19.1307 & & & 2.4 & 2.4 & 2.0 & 1.7 & 8.5 & 6.1 \\
\hline orf19.4144 & & & 2.4 & 3.2 & 1.2 & 1.6 & 0.9 & 0.9 \\
\hline orf19.147 & $Y A K 1$ & filamentous growth & 2.4 & 2.4 & 1.2 & 2.0 & 2.3 & 1.7 \\
\hline orf19.501 & & rRNA processing & 2.4 & 3.3 & 1.0 & 1.4 & 3.2 & 2.3 \\
\hline orf19.6548 & ISU1 & cellular iron ion homeostasis & 2.4 & 2.1 & 4.8 & 6.5 & 7.7 & 5.8 \\
\hline orf19.5282 & & & 2.4 & 2.7 & 0.5 & 0.4 & 1.9 & 5.4 \\
\hline orf19.7578 & & ER to Golgi vesicle-mediated transport & 2.3 & 2.5 & 1.8 & 2.1 & 3.2 & 3.2 \\
\hline orf19.1546 & & & 2.3 & 1.5 & 1.8 & 1.5 & 0.4 & 0.9 \\
\hline orf19.6842 & TUS1 & fungal-type cell wall organization & 2.3 & 3.0 & 1.6 & 1.6 & 3.8 & 2.6 \\
\hline orf19.4815 & YTM1 & filamentous growth & 2.3 & 3.2 & 0.6 & 1.4 & 3.2 & 2.8 \\
\hline orf19.3135 & & ER-associated protein catabolic process & 2.3 & 2.6 & 1.2 & 1.1 & 1.7 & 1.6 \\
\hline orf19.2938 & & mitochondrial respiratory chain complex III assembly & 2.3 & 1.7 & 1.8 & 2.7 & 1.7 & 1.2 \\
\hline orf19.5961 & & proteasome regulatory particle assembly & 2.3 & 2.0 & 1.3 & 1.2 & 1.7 & 3.1 \\
\hline orf19.2423 & ZCF11 & filamentous growth & 2.3 & 2.1 & 2.5 & 3.8 & 2.1 & 1.9 \\
\hline orf19.6557 & & & 2.3 & 2.1 & 1.4 & 1.4 & 1.7 & 2.1 \\
\hline orf19.2527 & & single-species biofilm formation on inanimate substrate & 2.3 & 2.3 & 1.7 & 3.0 & 3.0 & 4.8 \\
\hline orf19.2876 & $C B F 1$ & chromosome segregation & 2.3 & 3.2 & 0.5 & 0.5 & 0.4 & 2.0 \\
\hline orf19.6953 & IRS4 & fungal-type cell wall organization & 2.3 & 2.0 & 4.5 & 5.7 & 14.3 & 7.6 \\
\hline orf19.7247 & RIM101 & cellular response to iron ion starvation & 2.3 & 3.4 & 4.7 & 6.9 & 10.1 & 6.0 \\
\hline
\end{tabular}


Table A-1. (Continued)

\begin{tabular}{|c|c|c|c|c|c|c|c|c|}
\hline \multirow[b]{3}{*}{$\begin{array}{c}\text { orf19 } \\
\text { Designation }\end{array}$} & \multirow[b]{3}{*}{$\begin{array}{l}\text { CGD } \\
\text { Name }\end{array}$} & \multirow[b]{3}{*}{ Process $^{a}$} & \multicolumn{6}{|c|}{ Change in Fold Expression } \\
\hline & & & \multicolumn{2}{|c|}{$\begin{array}{c}\text { SC5314+FLC } \\
\text { /SC5314 } \\
\end{array}$} & \multicolumn{2}{|c|}{$\begin{array}{c}\operatorname{cas} 5 \Delta / \Delta+\mathrm{FLC} \\
/ \operatorname{cas} 5 \Delta / \Delta \\
\end{array}$} & \multicolumn{2}{|c|}{$\begin{array}{c}\text { upc } 2 \Delta / \Delta+\mathrm{FLC} / \\
u p c 2 \Delta / \Delta\end{array}$} \\
\hline & & & Exp1 & Exp2 & Exp1 & Exp2 & Exp1 & Exp2 \\
\hline orf19.3746 & IFC1 & oligopeptide transport & 2.3 & 2.7 & 1.3 & 0.6 & 0.2 & 0.0 \\
\hline orf19.6376 & PTC5 & protein amino acid dephosphorylation & 2.3 & 3.6 & 2.4 & 2.9 & 3.3 & 2.0 \\
\hline orf19.2210 & & & 2.3 & 4.3 & 1.0 & 1.9 & 8.0 & 3.8 \\
\hline orf19.3169 & & establishment of mitotic sister chromatid cohesion & 2.3 & 2.2 & 1.5 & 1.5 & 2.6 & 3.2 \\
\hline orf19.6938 & $M E U 1$ & glutamate biosynthetic process & 2.3 & 2.3 & 1.8 & 2.8 & 2.7 & 1.4 \\
\hline orf19.6971 & DIE2 & protein amino acid N-linked glycosylation & 2.3 & 2.1 & 1.2 & 1.4 & 1.7 & 1.3 \\
\hline orf19.6026 & $E R G 2$ & ergosterol biosynthetic process & 2.3 & 1.5 & 2.6 & 4.7 & 1.3 & 0.4 \\
\hline orf19.1557 & & protein methylation & 2.3 & 2.0 & 1.1 & 1.1 & 2.5 & 3.0 \\
\hline orf19.1939 & & & 2.3 & 1.6 & 0.6 & 0.8 & 3.3 & 1.9 \\
\hline orf19.5552 & & regulation of transcription from RNA polymerase II promoter & 2.3 & 3.7 & 1.7 & 1.8 & 3.5 & 2.7 \\
\hline orf19.345 & & response to oxidative stress & 2.3 & 1.9 & 5.6 & 4.8 & 1.3 & 1.4 \\
\hline orf19.951 & & & 2.3 & 2.4 & 0.4 & 0.4 & 0.8 & 1.2 \\
\hline orf19.5713 & $Y M X 6$ & glucose catabolic process to ethanol & 2.3 & 1.8 & 1.8 & 1.6 & 2.2 & 1.8 \\
\hline orf19.5611 & & ergosterol metabolic process & 2.3 & 1.5 & 12.7 & 8.9 & 4.9 & 10.2 \\
\hline orf19.2917 & & rRNA processing & 2.3 & 2.3 & 1.3 & 2.9 & 4.0 & 3.2 \\
\hline orf19.3921 & & & 2.3 & 2.1 & 1.2 & 1.6 & 1.8 & 2.0 \\
\hline orf19.6624 & & regulation of Rab GTPase activity & 2.3 & 2.2 & 3.0 & 5.8 & 2.9 & 2.3 \\
\hline orf19.2962 & & & 2.3 & 2.2 & 0.7 & 0.8 & 33.6 & 13.7 \\
\hline orf19.1531 & & mRNA cleavage & 2.3 & 1.8 & 1.1 & 1.5 & 2.2 & 2.0 \\
\hline orf19.3726 & & & 2.3 & 2.2 & 1.6 & 2.6 & 1.6 & 1.7 \\
\hline orf19.7550 & $I F A 14$ & & 2.3 & 1.7 & 1.0 & 0.7 & 4.0 & 9.6 \\
\hline orf19.5644 & & ascospore wall assembly & 2.3 & 3.5 & 1.1 & 1.0 & 2.7 & 1.9 \\
\hline orf19.6385 & $A C O 1$ & citrate metabolic process & 2.3 & 2.7 & 1.9 & 2.6 & 2.4 & 1.4 \\
\hline orf19.2079 & PHHB & filamentous growth & 2.3 & 2.9 & 1.3 & 1.3 & 1.8 & 5.7 \\
\hline orf19.2030 & & & 2.3 & 2.3 & 2.9 & 2.7 & 4.7 & 2.8 \\
\hline orf19.3732 & $E R G 25$ & ergosterol biosynthetic process & 2.3 & 1.5 & 4.4 & 6.5 & 7.9 & 2.4 \\
\hline orf19.4411 & HOS1 & chromatin organization & 2.3 & 2.2 & 1.6 & 1.7 & 3.0 & 2.7 \\
\hline
\end{tabular}


Table A-1. (Continued)

\begin{tabular}{|c|c|c|c|c|c|c|c|c|}
\hline \multirow[b]{3}{*}{$\begin{array}{c}\text { orf19 } \\
\text { Designation }\end{array}$} & \multirow[b]{3}{*}{$\begin{array}{l}\text { CGD } \\
\text { Name }\end{array}$} & \multirow[b]{3}{*}{ Process $^{\mathrm{a}}$} & \multicolumn{6}{|c|}{ Change in Fold Expression } \\
\hline & & & \multicolumn{2}{|c|}{$\begin{array}{c}\text { SC5314+FLC } \\
\text { /SC5314 } \\
\end{array}$} & \multicolumn{2}{|c|}{$\begin{array}{c}\operatorname{cas} 5 \Delta / \Delta+F L C \\
\quad / c a s 5 \Delta / \Delta \\
\end{array}$} & \multicolumn{2}{|c|}{$\begin{array}{c}\text { upc } 2 \Delta / \Delta+\mathrm{FLC} / \\
u p c 2 \Delta / \Delta\end{array}$} \\
\hline & & & Exp1 & Exp2 & Exp1 & Exp2 & Exp1 & Exp2 \\
\hline orf19.415 & & cristae formation & 2.2 & 2.3 & 1.7 & 2.1 & 1.2 & 1.0 \\
\hline orf19.6411 & & phospholipid metabolic process & 2.2 & 2.8 & 1.7 & 1.3 & 2.3 & 1.6 \\
\hline orf19.1839 & $R P A 190$ & transcription from RNA polymerase I promoter & 2.2 & 4.6 & 1.3 & 2.2 & 2.9 & 1.8 \\
\hline orf19.3675 & GAL7 & galactose catabolic process via UDP-galactose & 2.2 & 1.9 & 2.0 & 1.8 & 2.0 & 0.9 \\
\hline orf19.899 & & & 2.2 & 1.9 & 1.8 & 1.9 & 4.2 & 3.2 \\
\hline orf19.2003 & $H N M 1$ & choline transport & 2.2 & 1.9 & 2.5 & 3.8 & 7.1 & 1.7 \\
\hline orf19.706 & NMD3 & ribosomal large subunit assembly & 2.2 & 3.1 & 1.8 & 3.3 & 4.7 & 3.3 \\
\hline orf19.5131 & & negative regulation of gluconeogenesis & 2.2 & 2.5 & 1.5 & 1.0 & 2.8 & 2.9 \\
\hline orf19.6578 & & phosphate transport & 2.2 & 3.1 & 3.5 & 3.0 & 2.0 & 0.9 \\
\hline orf19.3150 & GRE2 & response to osmotic stress & 2.2 & 1.6 & 3.9 & 2.9 & 2.4 & 2.5 \\
\hline orf19.7011 & & maturation of SSU-rRNA & 2.2 & 4.7 & 1.1 & 2.3 & 4.8 & 3.9 \\
\hline orf19.944 & $I F G 3$ & oxidation-reduction process & 2.2 & 2.6 & 4.0 & 4.4 & 5.1 & 2.6 \\
\hline orf19.6713 & & & 2.2 & 3.7 & 2.7 & 2.5 & 0.9 & 0.3 \\
\hline orf19.6229 & CAT1 & hyphal growth & 2.2 & 1.5 & 5.5 & 6.1 & 4.9 & 2.2 \\
\hline orf19.4970 & & & 2.2 & 4.9 & 2.5 & 1.6 & 228.3 & 47.3 \\
\hline orf19.7513 & $A L K 2$ & oxidation-reduction process & 2.2 & 3.5 & 4.5 & 6.4 & 40.9 & 20.2 \\
\hline orf19.4584 & PHO114 & phosphate metabolic process & 2.2 & 1.5 & 2.3 & 2.1 & 2.4 & 1.9 \\
\hline orf19.2204.2 & & cell wall organization & 2.2 & 2.6 & 1.4 & 1.5 & 2.8 & 1.8 \\
\hline orf19.3444 & & drug transport & 2.2 & 3.3 & 1.6 & 1.2 & 2.2 & 3.1 \\
\hline orf19.2575 & & & 2.2 & 2.3 & 1.1 & 1.9 & 2.0 & 2.5 \\
\hline orf19.4795 & & & 2.2 & 2.6 & 3.4 & 4.8 & 11.5 & 5.1 \\
\hline orf19.3724 & & endonucleolytic cleavage of rRNA & 2.2 & 1.7 & 3.0 & 4.0 & 1.4 & 2.7 \\
\hline orf19.7320 & $L I P 7$ & lipid catabolic process & 2.2 & 2.5 & 3.6 & 3.8 & 16.6 & 9.0 \\
\hline orf19.669 & PRM1 & plasma membrane fusion during cytogamy & 2.2 & 2.8 & 0.6 & 0.4 & 1.2 & 3.2 \\
\hline orf19.3245 & & & 2.2 & 2.3 & 1.2 & 1.1 & 2.5 & 1.6 \\
\hline orf19.7472 & IFF4 & cell-substrate adhesion & 2.2 & 2.3 & 1.6 & 1.7 & 4.3 & 2.2 \\
\hline orf19.318 & & iron-sulfur cluster assembly & 2.2 & 2.0 & 2.9 & 4.5 & 2.0 & 1.6 \\
\hline
\end{tabular}


Table A-1. (Continued)

\begin{tabular}{|c|c|c|c|c|c|c|c|c|}
\hline \multirow[b]{3}{*}{$\begin{array}{c}\text { orf19 } \\
\text { Designation }\end{array}$} & \multirow[b]{3}{*}{$\begin{array}{c}\text { CGD } \\
\text { Name }\end{array}$} & \multirow[b]{3}{*}{ Process $^{\mathrm{a}}$} & \multicolumn{6}{|c|}{ Change in Fold Expression } \\
\hline & & & \multicolumn{2}{|c|}{$\begin{array}{c}\text { SC5314+FLC } \\
\text { /SC5314 } \\
\end{array}$} & \multicolumn{2}{|c|}{$\begin{array}{c}\operatorname{cas} 5 \Delta / \Delta+\mathrm{FLC} \\
/ \operatorname{cas} 5 \Delta / \Delta\end{array}$} & \multicolumn{2}{|c|}{$\begin{array}{c}u p c 2 \Delta / \Delta+\mathrm{FLC} / \\
u p c 2 \Delta / \Delta\end{array}$} \\
\hline & & & Exp1 & Exp2 & Exp1 & Exp2 & Exp1 & Exp2 \\
\hline orf19.2781 & & protein amino acid phosphorylation & 2.2 & 2.1 & 4.6 & 2.5 & 27.0 & 4.6 \\
\hline orf19.7546 & & maturation of rRNA) & 2.2 & 1.5 & 2.8 & 5.3 & 8.7 & 5.0 \\
\hline orf19.4591 & CAT2 & acetyl-CoA metabolic process & 2.2 & 2.6 & 4.3 & 6.5 & 5.2 & 2.8 \\
\hline orf19.188 & & & 2.2 & 2.0 & 6.1 & 4.8 & 0.9 & 1.7 \\
\hline orf19.5640 & $P E X 5$ & fatty acid beta-oxidation & 2.2 & 2.6 & 0.9 & 0.9 & 2.2 & 2.3 \\
\hline orf19.7618 & & endonucleolytic cleavageof rRNA & 2.2 & 1.6 & 1.3 & 3.1 & 9.9 & 7.4 \\
\hline orf19.6956 & $D A L 9$ & anion transport & 2.2 & 2.5 & 1.6 & 1.8 & 1.6 & 1.3 \\
\hline orf19.2091 & & oxidation-reduction process & 2.2 & 2.3 & 1.9 & 3.4 & 0.8 & 0.9 \\
\hline orf19.732 & & fatty acid catabolic process & 2.2 & 2.2 & 2.4 & 4.1 & 14.2 & 21.9 \\
\hline orf19.2726 & & actin cytoskeleton organization & 2.2 & 1.5 & 6.0 & 5.0 & 7.2 & 3.7 \\
\hline orf19.1902 & NOC4 & endonucleolytic cleavage of rRNA & 2.2 & 1.9 & 1.6 & 3.0 & 2.1 & 4.3 \\
\hline orf19.1701 & RIK1 & pyridoxine biosynthetic process & 2.2 & 1.5 & 4.4 & 4.6 & 6.5 & 3.6 \\
\hline orf19.3863 & & & 2.2 & 28.3 & 6.2 & 35.3 & 157.2 & 36.4 \\
\hline orf19.7359 & $C R Z 1$ & calcium-mediated signaling & 2.1 & 3.3 & 3.3 & 6.4 & 5.9 & 2.6 \\
\hline orf19.3885 & & metabolic process & 2.1 & 2.2 & 4.3 & 8.3 & 75.7 & 17.7 \\
\hline orf19.6594 & $P L B 3$ & phosphatidylserine catabolic process & 2.1 & 3.4 & 1.1 & 0.9 & 3.4 & 2.8 \\
\hline orf19.7204 & & oxidation-reduction process & 2.1 & 2.5 & 2.1 & 2.3 & 3.3 & 4.3 \\
\hline orf19.5610 & $A R G 3$ & arginine biosynthetic process & 2.1 & 1.7 & 4.2 & 5.2 & 2.0 & 1.3 \\
\hline orf19.2175 & & positive regulation of apoptosis & 2.1 & 2.7 & 1.8 & 1.4 & 1.4 & 2.3 \\
\hline orf19.6706 & $G Y P 7$ & vesicle-mediated transport & 2.1 & 2.4 & 1.9 & 1.8 & 3.0 & 2.3 \\
\hline orf19.2176 & IFM3 & oxidation-reduction process & 2.1 & 1.8 & 1.7 & 2.1 & 3.6 & 3.6 \\
\hline orf19.3621 & & & 2.1 & 3.5 & 1.3 & 0.7 & 3.7 & 3.1 \\
\hline orf19.5426 & & cell budding & 2.1 & 2.5 & 2.3 & 2.4 & 5.9 & 4.3 \\
\hline orf19.7061 & & metabolic process & 2.1 & 1.7 & 1.2 & 1.3 & 1.0 & 2.1 \\
\hline orf19.4012 & PCL5 & filamentous growth & 2.1 & 6.0 & 3.4 & 3.2 & 13.1 & 7.5 \\
\hline orf19.5198 & NOP4 & rRNA processing & 2.1 & 1.8 & 2.0 & 3.1 & 1.1 & 3.5 \\
\hline orf19.5601 & & & 2.1 & 2.0 & 2.5 & 2.2 & 1.6 & 2.6 \\
\hline
\end{tabular}


Table A-1. (Continued)

\begin{tabular}{|c|c|c|c|c|c|c|c|c|}
\hline \multirow{3}{*}{$\begin{array}{c}\text { orf19 } \\
\text { Designation }\end{array}$} & \multirow{3}{*}{$\begin{array}{l}\text { CGD } \\
\text { Name }\end{array}$} & \multirow[b]{3}{*}{ Process $^{\mathrm{a}}$} & \multicolumn{6}{|c|}{ Change in Fold Expression } \\
\hline & & & \multicolumn{2}{|c|}{$\begin{array}{c}\text { SC5314+FLC } \\
\text { /SC5314 } \\
\end{array}$} & \multicolumn{2}{|c|}{$\begin{array}{c}\operatorname{cas} 5 \Delta / \Delta+\mathrm{FLC} \\
/ \operatorname{cas} 5 \Delta / \Delta \\
\end{array}$} & \multicolumn{2}{|c|}{$\begin{array}{c}u p c 2 \Delta / \Delta+\mathrm{FLC} / \\
u p c 2 \Delta / \Delta\end{array}$} \\
\hline & & & Exp1 & Exp2 & Exp1 & Exp2 & Exp1 & Exp2 \\
\hline orf19.1682 & & & 2.1 & 3.0 & 1.7 & 2.1 & 1.5 & 1.3 \\
\hline orf19.4229 & & diadenosine polyphosphate catabolic process & 2.1 & 2.0 & 3.0 & 2.9 & 3.3 & 4.4 \\
\hline orf19.2062 & SOD4 & evasion or tolerance & 2.1 & 3.2 & 3.3 & 3.6 & 3.6 & 4.9 \\
\hline orf19.1440 & & & 2.1 & 2.6 & 1.7 & 2.4 & 0.8 & 0.9 \\
\hline orf19.7517 & CHT1 & cytokinesis, completion of separation & 2.1 & 2.5 & 1.8 & 1.5 & 14.0 & 3.4 \\
\hline orf19.6811 & & iron ion transport & 2.1 & 1.8 & 2.0 & 1.9 & 3.1 & 1.9 \\
\hline orf19.4982 & & lipid metabolic process & 2.1 & 2.3 & 0.9 & 0.6 & 0.7 & 0.8 \\
\hline orf19.2192 & $G D H 2$ & nitrogen compound metabolic process & 2.1 & 1.9 & 1.5 & 2.6 & 2.4 & 1.1 \\
\hline orf19.3154 & & & 2.1 & 2.3 & 2.0 & 3.3 & 0.6 & 0.6 \\
\hline orf19.692 & & & 2.1 & 1.9 & 1.6 & 1.9 & 3.2 & 4.2 \\
\hline orf19.5958 & $C D R 2$ & drug export & 2.1 & 2.5 & 0.9 & 1.0 & 2.5 & 1.7 \\
\hline orf19.6983 & & & 2.1 & 9.1 & 1.0 & 1.3 & 0.4 & 0.0 \\
\hline orf19.124 & CIC1 & protein catabolic process & 2.1 & 2.0 & 1.5 & 3.8 & 1.7 & 4.2 \\
\hline orf19.6132 & & & 2.1 & 2.0 & 1.7 & 2.1 & 1.4 & 1.7 \\
\hline orf19.1482 & $H S K 3$ & mitotic spindle organization in nucleus & 2.1 & 1.6 & 2.3 & 1.7 & 1.1 & 1.3 \\
\hline orf19.1300 & & filamentous growth & 2.1 & 1.8 & 2.1 & 2.3 & 0.3 & 0.6 \\
\hline orf19.4090 & & fucose transport & 2.1 & 1.7 & 1.1 & 0.8 & 3.8 & 4.5 \\
\hline orf19.5845 & $R N R 3$ & DNA replication & 2.1 & 2.7 & 4.5 & 5.3 & 1.4 & 1.2 \\
\hline orf19.4278 & & negative regulation of transcription from RNA pol II promoter & 2.1 & 2.3 & 1.8 & 1.8 & 2.4 & 1.9 \\
\hline orf19.6902 & $D B P 7$ & ribosomal large subunit assembly & 2.1 & 2.0 & 1.0 & 2.0 & 9.9 & 10.3 \\
\hline orf19.6139 & FRE7 & siderophore-iron transport & 2.1 & 4.9 & 9.5 & 7.8 & 37.7 & 25.9 \\
\hline orf19.2825 & & iron-sulfur cluster assembly & 2.1 & 1.5 & 2.1 & 1.9 & 1.5 & 2.2 \\
\hline orf19.4862 & & vesicle-mediated transport & 2.1 & 1.6 & 1.5 & 1.8 & 3.9 & 2.1 \\
\hline orf19.5089 & TERT & telomere capping & 2.1 & 2.3 & 1.8 & 1.6 & 3.1 & 1.4 \\
\hline orf19.272 & $F A A 21$ & lipid metabolic process & 2.1 & 3.0 & 6.6 & 8.3 & 11.5 & 7.4 \\
\hline orf19.1549 & & & 2.1 & 1.8 & 2.0 & 3.0 & 1.1 & 1.6 \\
\hline orf19.4215 & FET34 & high-affinity iron ion transport & 2.1 & 5.2 & 0.2 & 0.5 & 0.0 & 0.1 \\
\hline
\end{tabular}


Table A-1. (Continued)

\begin{tabular}{|c|c|c|c|c|c|c|c|c|}
\hline \multirow[b]{3}{*}{$\begin{array}{c}\text { orf19 } \\
\text { Designation }\end{array}$} & \multirow[b]{3}{*}{$\begin{array}{l}\text { CGD } \\
\text { Name }\end{array}$} & \multirow[b]{3}{*}{ Process $^{\mathrm{a}}$} & \multicolumn{6}{|c|}{ Change in Fold Expression } \\
\hline & & & \multicolumn{2}{|c|}{$\begin{array}{c}\text { SC5314+FLC } \\
\text { /SC5314 }\end{array}$} & \multicolumn{2}{|c|}{$\begin{array}{l}\operatorname{cas} 5 \Delta / \Delta+F L C \\
/ \operatorname{cas} 5 \Delta / \Delta\end{array}$} & \multicolumn{2}{|c|}{$\begin{array}{c}u p c 2 \Delta / \Delta+\mathrm{FLC} / \\
u p c 2 \Delta / \Delta\end{array}$} \\
\hline & & & Exp1 & Exp2 & Exp1 & Exp2 & Exp1 & $\operatorname{Exp2}$ \\
\hline orf19.5924 & ZCF31 & cellular response to drug & 2.1 & 2.3 & 1.8 & 1.9 & 2.5 & 2.0 \\
\hline orf19.1426 & & & 2.1 & 2.8 & 2.5 & 3.5 & 2.6 & 1.0 \\
\hline orf19.6517 & $R A D 14$ & nucleotide-excision repair, DNA damage recognition & 2.1 & 1.7 & 2.7 & 3.2 & 2.8 & 2.6 \\
\hline orf19.26 & & misfolded or incompletely synthesized protein catabolic process & 2.1 & 2.0 & 1.5 & 1.8 & 1.8 & 2.1 \\
\hline orf19.5492 & & nuclear mRNA splicing, via spliceosome & 2.1 & 1.9 & 2.2 & 3.9 & 1.5 & 3.2 \\
\hline orf19.396 & EAF6 & histone acetylation & 2.1 & 1.5 & 1.3 & 1.2 & 0.7 & 1.4 \\
\hline orf19.3611 & & & 2.1 & 2.2 & 2.1 & 3.1 & 1.1 & 1.4 \\
\hline orf19.7512 & & oxidation-reduction process & 2.1 & 1.8 & 1.5 & 1.6 & 5.2 & 3.0 \\
\hline orf19.500 & & tRNA methylation & 2.1 & 2.3 & 1.5 & 2.6 & 2.9 & 2.6 \\
\hline orf19.2202 & & & 2.1 & 1.8 & 5.5 & 6.4 & 15.1 & 10.1 \\
\hline orf19.1813 & $F L C 2$ & heme transport & 2.1 & 1.7 & 2.5 & 3.0 & 7.1 & 1.9 \\
\hline orf19.3669 & SHA3 & filamentous growth & 2.1 & 2.4 & 1.6 & 2.9 & 4.5 & 2.5 \\
\hline orf19.5097 & CAT8 & filamentous growth & 2.1 & 1.7 & 3.6 & 4.8 & 8.1 & 4.7 \\
\hline orf19.795 & VPS36 & hyphal growth & 2.0 & 2.5 & 0.6 & 0.6 & 0.9 & 1.5 \\
\hline orf19.7385 & & & 2.0 & 2.4 & 1.3 & 1.7 & 85.7 & 31.9 \\
\hline orf19.4975 & HYR1 & single-species biofilm formation in or on host organism & 2.0 & 1.8 & 1.4 & 1.5 & 4.0 & 2.7 \\
\hline orf19.5677 & DUR4 & urea transport & 2.0 & 2.5 & 1.2 & 0.5 & 2.8 & 3.6 \\
\hline orf19.6268 & & & 2.0 & 1.8 & 1.9 & 2.0 & 1.9 & 1.5 \\
\hline orf19.7222 & PAM16 & protein import into mitochondrial matrix & 2.0 & 1.6 & 1.6 & 2.1 & 1.8 & 2.1 \\
\hline orf19.7436 & $A A F 1$ & cell adhesion & 2.0 & 4.4 & 1.0 & 1.2 & 2.1 & 1.2 \\
\hline orf19.6178 & FBP 1 & gluconeogenesis & 2.0 & 2.0 & 1.7 & 1.7 & 2.3 & 4.1 \\
\hline orf19.3127 & $C Z F 1$ & filamentous growth & 2.0 & 2.9 & 2.8 & 2.9 & 3.2 & 2.0 \\
\hline orf19.1114 & & mitochondrial respiratory chain complex IV assembly & 2.0 & 1.9 & 2.5 & 3.3 & 3.5 & 3.6 \\
\hline orf19.6274 & PBR1 & biofilm formation & 2.0 & 1.7 & 1.0 & 2.7 & 2.3 & 1.2 \\
\hline orf19.5270 & & & 2.0 & 2.3 & 1.4 & 1.8 & 2.5 & 5.4 \\
\hline orf19.2667 & $R P F 1$ & maturation of rRNA & 2.0 & 1.8 & 1.0 & 2.0 & 1.9 & 4.5 \\
\hline orf19.1270 & FRE3 & oxidation-reduction process & 2.0 & 1.5 & 1.2 & 1.9 & 48.0 & 7.4 \\
\hline
\end{tabular}


Table A-1. (Continued)

\begin{tabular}{|c|c|c|c|c|c|c|c|c|}
\hline \multirow[b]{3}{*}{$\begin{array}{c}\text { orf19 } \\
\text { Designation }\end{array}$} & \multirow[b]{3}{*}{$\begin{array}{c}\text { CGD } \\
\text { Name }\end{array}$} & \multirow[b]{3}{*}{ Process $^{\mathbf{a}}$} & \multicolumn{6}{|c|}{ Change in Fold Expression } \\
\hline & & & \multicolumn{2}{|c|}{$\begin{array}{c}\text { SC5314+FLC } \\
\text { /SC5314 } \\
\end{array}$} & \multicolumn{2}{|c|}{$\begin{array}{c}\operatorname{cas} 5 \Delta / \Delta+\mathrm{FLC} \\
/ \operatorname{cas} 5 \Delta / \Delta \\
\end{array}$} & \multicolumn{2}{|c|}{$\begin{array}{c}\text { upc } 2 \Delta / \Delta+\mathrm{FLC} / \\
u p c 2 \Delta / \Delta\end{array}$} \\
\hline & & & Exp1 & Exp2 & Exp1 & Exp2 & Exp1 & Exp2 \\
\hline orf19.1633 & UTP4 & maturation of rRNA & 2.0 & 1.5 & 1.2 & 3.5 & 0.9 & 1.6 \\
\hline orf19.4121 & & fatty acid metabolic process & 2.0 & 2.4 & 5.6 & 4.4 & 10.9 & 15.2 \\
\hline orf19.4356 & HGT3 & carbohydrate transport & 2.0 & 1.9 & 2.6 & 2.1 & 3.4 & 1.5 \\
\hline orf19.1264 & CFL2 & cellular iron ion homeostasis & 2.0 & 6.0 & 0.6 & 0.5 & 0.2 & 0.8 \\
\hline orf19.6518 & & oxidation-reduction process & 2.0 & 2.6 & 2.9 & 2.3 & 83.1 & 56.9 \\
\hline orf19.512 & & ribosomal small subunit biogenesis & 2.0 & 5.4 & 1.5 & 2.3 & 3.5 & 2.3 \\
\hline orf19.3578 & & & 2.0 & 1.5 & 1.7 & 4.1 & 4.1 & 5.8 \\
\hline orf19.5824 & & & 2.0 & 1.9 & 1.9 & 5.1 & 3.3 & 3.9 \\
\hline orf19.3151 & & cellular metabolic process & 2.0 & 1.9 & 2.3 & 1.8 & 3.1 & 2.7 \\
\hline orf19.3478 & NIP7 & rRNA processing & 2.0 & 1.5 & 0.7 & 1.9 & 0.9 & 1.5 \\
\hline orf19.1710 & ALII & oxidation-reduction process & 2.0 & 1.9 & 1.9 & 2.7 & 0.7 & 0.8 \\
\hline orf19.6928 & $S A P 9$ & fungal-type cell wall organization & 2.0 & 2.3 & 3.2 & 2.6 & 2.1 & 1.2 \\
\hline orf19.3338 & & & 2.0 & 4.0 & 3.4 & 5.1 & 5.2 & 13.2 \\
\hline orf19.4928 & $S E C 2$ & exocytosis & 2.0 & 2.1 & 0.4 & 0.4 & 1.1 & 2.6 \\
\hline orf19.2397.3 & & filamentous growth & 2.0 & 4.3 & 0.9 & 1.2 & 3.3 & 5.9 \\
\hline orf19.7282 & PEX13 & protein import into peroxisome matrix & 2.0 & 1.6 & 2.6 & 2.0 & 2.3 & 2.1 \\
\hline orf19.7006 & & reciprocal meiotic recombination & 2.0 & 3.1 & 1.5 & 2.1 & 4.2 & 2.1 \\
\hline orf19.4766 & $A R G 81$ & arginine metabolic process & 2.0 & 1.9 & 1.6 & 1.7 & 1.3 & 1.4 \\
\hline orf19.1598 & $E R G 24$ & ergosterol biosynthetic process & 2.0 & 1.6 & 2.2 & 2.2 & 0.9 & 0.7 \\
\hline orf19.5231.2 & ATP19 & ATP synthesis coupled proton transport & 2.0 & 2.2 & 1.3 & 1.9 & 0.7 & 1.4 \\
\hline orf19.5815 & SCT2 & phospholipid biosynthetic process & 2.0 & 2.3 & 1.3 & 1.1 & 2.8 & 1.5 \\
\hline orf19.1415 & FRE10 & iron ion transport & 2.0 & 4.2 & 0.9 & 2.3 & 0.2 & 0.1 \\
\hline orf19.1030 & & meiotic recombination checkpoint & 2.0 & 2.4 & 0.9 & 1.4 & 1.0 & 5.4 \\
\hline orf19.1224 & FRP3 & mmonium transport & 2.0 & 1.5 & 0.9 & 1.3 & 2.5 & 1.4 \\
\hline orf19.5255 & $P X A 2$ & fatty acid transport & 2.0 & 1.5 & 3.3 & 5.6 & 8.9 & 4.3 \\
\hline orf19.2622 & YPT31 & exocytosis & 2.0 & 1.7 & 2.1 & 3.2 & 1.4 & 1.8 \\
\hline orf19.1140 & & & 2.0 & 1.5 & 1.6 & 2.1 & 1.0 & 1.4 \\
\hline
\end{tabular}


Table A-1. (Continued)

\begin{tabular}{|c|c|c|c|c|c|c|c|c|}
\hline \multirow[b]{3}{*}{$\begin{array}{c}\text { orf19 } \\
\text { Designation }\end{array}$} & \multirow[b]{3}{*}{$\begin{array}{c}\text { CGD } \\
\text { Name }\end{array}$} & \multirow[b]{3}{*}{ Process $^{a}$} & \multicolumn{6}{|c|}{ Change in Fold Expression } \\
\hline & & & \multicolumn{2}{|c|}{$\begin{array}{c}\text { SC5314+FLC } \\
\text { /SC5314 } \\
\end{array}$} & \multicolumn{2}{|c|}{$\begin{array}{c}\operatorname{cas} 5 \Delta / \Delta+\mathrm{FLC} \\
/ \operatorname{cas} 5 \Delta / \Delta\end{array}$} & \multicolumn{2}{|c|}{$\begin{array}{c}u p c 2 \Delta / \Delta+\mathrm{FLC} / \\
u p c 2 \Delta / \Delta\end{array}$} \\
\hline & & & Exp1 & Exp2 & Exp1 & $\operatorname{Exp2}$ & Exp1 & Exp2 \\
\hline orf19.3412 & $A T G 15$ & multivesicular body membrane disassembly & 2.0 & 2.6 & 0.7 & 1.0 & 3.2 & 2.5 \\
\hline orf19.4187 & & mitochondrial genome maintenance & 2.0 & 1.8 & 1.5 & 1.2 & 3.2 & 4.3 \\
\hline orf19.7062 & $R P A 135$ & transcription from RNA polymerase I promoter & 2.0 & 5.6 & 0.9 & 1.6 & 3.8 & 2.7 \\
\hline orf19.6068 & SVF1 & response to oxidative stress & 2.0 & 2.0 & 2.5 & 4.7 & 4.0 & 2.4 \\
\hline orf19.73 & & proteolysis & 2.0 & 1.7 & 7.6 & 5.4 & 14.4 & 3.9 \\
\hline orf19.2998 & $T S R 2$ & maturation of rRNA & 2.0 & 2.4 & 1.5 & 2.6 & 2.5 & 3.5 \\
\hline orf19.2650 & & & 2.0 & 1.5 & 1.6 & 2.0 & 1.2 & 1.5 \\
\hline orf19.2016 & & ergosterol biosynthetic process & 2.0 & 2.0 & 1.3 & 1.4 & 1.0 & 1.5 \\
\hline orf19.2570 & MCI4 & respiratory electron transport chain & 2.0 & 1.7 & 1.2 & 2.0 & 1.5 & 2.1 \\
\hline orf19.7107 & & ribosomal large subunit biogenesis & 2.0 & 2.2 & 0.6 & 1.2 & 3.2 & 3.3 \\
\hline orf19.7261 & & vesicle-mediated transport & 2.0 & 2.3 & 1.8 & 1.8 & 2.7 & 1.9 \\
\hline orf19.994 & & & 2.0 & 2.7 & 0.7 & 1.3 & 3.4 & 1.7 \\
\hline orf19.7316 & & response to drug & 2.0 & 2.3 & 0.7 & 0.8 & 2.6 & 2.2 \\
\hline orf19.5491 & ATP14 & ATP synthesis coupled proton transport & 1.9 & 2.3 & 1.3 & 1.8 & 1.3 & 1.6 \\
\hline orf19.2005 & REG1 & glycogen metabolic process & 1.9 & 2.2 & 2.3 & 3.3 & 3.1 & 1.8 \\
\hline orf19.4792 & & termination of G-protein coupled receptor signaling pathway & 1.9 & 1.6 & 1.0 & 1.7 & 11.5 & 5.3 \\
\hline orf19.3617 & GTR1 & chromatin silencing at telomere & 1.9 & 2.3 & 1.5 & 1.7 & 1.1 & 3.4 \\
\hline orf19.5170 & ENA21 & sodium ion transport & 1.9 & 2.5 & 3.0 & 3.2 & 4.3 & 2.8 \\
\hline orf19.6225.1 & & mitochondrial respiratory chain complex IV assembly & 1.9 & 1.9 & 0.9 & 0.7 & 0.7 & 1.6 \\
\hline orf19.5006.1 & & Golgi to plasma membrane transport & 1.9 & 1.5 & 1.6 & 1.5 & 4.5 & 3.2 \\
\hline orf19.4746 & JIP5 & ribosomal large subunit biogenesis & 1.9 & 1.7 & 1.9 & 4.4 & 1.0 & 2.2 \\
\hline orf19.2115 & & & 1.9 & 2.8 & 0.9 & 1.5 & 4.6 & 1.7 \\
\hline orf19.4055 & & filamentous growth & 1.9 & 2.5 & 0.9 & 0.7 & 1.9 & 1.4 \\
\hline orf19.972 & & DNA repair & 1.9 & 2.9 & 0.8 & 0.6 & 2.1 & 2.4 \\
\hline orf19.4014 & & budding cell bud growth & 1.9 & 1.6 & 0.8 & 0.8 & 2.5 & 2.6 \\
\hline orf19.6881 & $Y T H 1$ & mRNA cleavage & 1.9 & 2.0 & 0.9 & 0.9 & 0.8 & 1.5 \\
\hline orf19.6686 & ENP2 & rRNA processing & 1.9 & 1.6 & 3.5 & 6.3 & 9.1 & 8.1 \\
\hline
\end{tabular}


Table A-1. (Continued)

\begin{tabular}{|c|c|c|c|c|c|c|c|c|}
\hline \multirow[b]{3}{*}{$\begin{array}{c}\text { orf19 } \\
\text { Designation }\end{array}$} & \multirow[b]{3}{*}{$\begin{array}{l}\text { CGD } \\
\text { Name }\end{array}$} & \multirow[b]{3}{*}{ Process $^{a}$} & \multicolumn{6}{|c|}{ Change in Fold Expression } \\
\hline & & & \multicolumn{2}{|c|}{$\begin{array}{c}\text { SC5314+FLC } \\
\text { /SC5314 } \\
\end{array}$} & \multicolumn{2}{|c|}{$\begin{array}{c}\operatorname{cas} 5 \Delta / \Delta+\mathrm{FLC} \\
/ \operatorname{cas} 5 \Delta / \Delta\end{array}$} & \multicolumn{2}{|c|}{$\begin{array}{c}\text { upc } 2 \Delta / \Delta+\mathrm{FLC} / \\
u p c 2 \Delta / \Delta\end{array}$} \\
\hline & & & Exp1 & Exp2 & Exp1 & $\operatorname{Exp2}$ & Exp1 & Exp2 \\
\hline orf19.4791 & & & 1.9 & 1.9 & 1.2 & 1.0 & 4.3 & 3.1 \\
\hline orf19.4016 & & aerobic respiration & 1.9 & 2.8 & 1.7 & 2.4 & 1.6 & 1.3 \\
\hline orf19.1250 & & maturation of rRNA & 1.9 & 1.5 & 1.5 & 2.3 & 2.2 & 3.6 \\
\hline orf19.6369 & RIO2 & maturation of rRNA & 1.9 & 1.9 & 1.4 & 2.1 & 1.9 & 2.2 \\
\hline orf19.1770 & $C Y C 1$ & mitochondrial electron transport, ubiquinol to cytochrome c & 1.9 & 2.2 & 1.4 & 1.7 & 0.6 & 0.7 \\
\hline orf19.4549 & $F G R 38$ & filamentous growth & 1.9 & 2.5 & 1.1 & 1.1 & 2.3 & 1.2 \\
\hline orf19.4941 & TYE7 & positive regulation of glycolysis & 1.9 & 1.9 & 1.3 & 1.5 & 1.5 & 1.3 \\
\hline orf19.4401 & $Y V H 1$ & hyphal growth & 1.9 & 1.8 & 1.1 & 2.3 & 2.7 & 3.8 \\
\hline orf19.909 & STP4 & regulation of transcription, DNA-dependent & 1.9 & 1.5 & 4.0 & 4.6 & 3.2 & 3.1 \\
\hline orf19.1791 & & maturation of rRNA & 1.9 & 2.5 & 1.7 & 3.0 & 0.9 & 1.5 \\
\hline orf19.2818 & & generation of catalytic spliceosome for second transesterification step & 1.9 & 1.6 & 1.8 & 3.4 & 5.2 & 2.6 \\
\hline orf19.7150 & $N R G 1$ & filamentous growth & 1.9 & 1.8 & 0.8 & 1.3 & 1.4 & 0.7 \\
\hline orf19.7034 & & negative regulation of translation & 1.9 & 2.1 & 3.3 & 3.7 & 1.5 & 1.1 \\
\hline orf19.6185 & & & 1.9 & 1.9 & 2.6 & 3.4 & 1.1 & 0.9 \\
\hline orf19.3778 & & ribosomal large subunit assembly & 1.9 & 2.5 & 0.7 & 1.4 & 3.0 & 2.4 \\
\hline orf19.1313 & $C D R 3$ & drug export & 1.9 & 2.1 & 0.9 & 1.0 & 2.4 & 1.7 \\
\hline orf19.5553 & & response to oxidative stress & 1.9 & 2.3 & 1.3 & 1.8 & 2.3 & 2.4 \\
\hline orf19.3241 & & & 1.9 & 2.0 & 1.9 & 1.8 & 3.4 & 2.2 \\
\hline orf19.2050 & & sterol metabolic process & 1.9 & 2.2 & 1.2 & 1.3 & 2.9 & 1.8 \\
\hline orf19.925 & SAM51 & protein complex assembly & 1.9 & 2.8 & 2.3 & 3.4 & 1.1 & 0.9 \\
\hline orf19.5367 & RDH54 & DNmeiotic sister chromatid segregation & 1.9 & 2.2 & 2.1 & 2.3 & 2.1 & 0.9 \\
\hline orf19.151 & TPO5 & ployamine transport & 1.9 & 1.9 & 0.9 & 1.1 & 2.8 & 3.3 \\
\hline orf19.2984 & MST1 & mitochondrial threonyl-tRNA aminoacylation & 1.9 & 2.0 & 1.0 & 1.1 & 0.7 & 1.0 \\
\hline orf19.6852 & & & 1.9 & 1.7 & 1.3 & 1.2 & 1.0 & 1.4 \\
\hline orf19.2517 & & drug transport & 1.9 & 1.6 & 2.0 & 1.8 & 2.3 & 2.0 \\
\hline orf19.4937 & $\mathrm{CHS} 3$ & cell wall chitin biosynthetic process & 1.9 & 2.0 & 1.9 & 2.5 & 6.3 & 2.4 \\
\hline orf19.753 & & protein O-linked glycosylation & 1.9 & 1.9 & 1.8 & 2.6 & 9.0 & 6.7 \\
\hline
\end{tabular}


Table A-1. (Continued)

\begin{tabular}{|c|c|c|c|c|c|c|c|c|}
\hline \multirow[b]{3}{*}{$\begin{array}{c}\text { orf19 } \\
\text { Designation }\end{array}$} & \multirow[b]{3}{*}{$\begin{array}{l}\text { CGD } \\
\text { Name }\end{array}$} & \multirow[b]{3}{*}{ Process $^{\mathrm{a}}$} & \multicolumn{6}{|c|}{ Change in Fold Expression } \\
\hline & & & \multicolumn{2}{|c|}{$\begin{array}{c}\text { SC5314+FLC } \\
\text { /SC5314 }\end{array}$} & \multicolumn{2}{|c|}{$\begin{array}{l}\operatorname{cas} 5 \Delta / \Delta+F L C \\
/ \operatorname{cas} 5 \Delta / \Delta\end{array}$} & \multicolumn{2}{|c|}{$\begin{array}{c}u p c 2 \Delta / \Delta+\mathrm{FLC} / \\
u p c 2 \Delta / \Delta\end{array}$} \\
\hline & & & Exp1 & Exp2 & Exp1 & Exp2 & Exp1 & Exp2 \\
\hline orf19.3228 & & pseudohyphal growth & 1.9 & 1.8 & 0.8 & 1.2 & 1.4 & 0.8 \\
\hline orf19.2906 & $P G A 41$ & & 1.9 & 1.6 & 1.5 & 1.0 & 4.9 & 2.3 \\
\hline orf19.1570 & $E R G 7$ & ergosterol biosynthetic process & 1.9 & 1.8 & 1.5 & 1.4 & 1.7 & 1.3 \\
\hline orf19.6460 & $P E X 1$ & protein import into peroxisome matrix, receptor recycling & 1.9 & 1.8 & 2.4 & 2.7 & 1.9 & 1.7 \\
\hline orf19.6727 & RIT1 & charged-tRNA amino acid modification & 1.9 & 2.2 & 1.1 & 1.1 & 1.9 & 3.4 \\
\hline orf19.1619 & & mRNA 3'-end processing & 1.9 & 2.7 & 3.7 & 2.7 & 1.7 & 1.6 \\
\hline orf19.5203 & & & 1.9 & 2.2 & 2.7 & 2.7 & 1.4 & 1.4 \\
\hline orf19.2446 & & pantothenate biosynthetic process & 1.9 & 2.0 & 2.4 & 2.4 & 2.6 & 2.8 \\
\hline orf19.6090 & & single-species biofilm formation on inanimate substrate & 1.9 & 2.4 & 0.6 & 0.6 & 0.9 & 4.5 \\
\hline orf19.3769 & & zinc ion transport & 1.9 & 1.6 & 1.7 & 2.4 & 6.4 & 3.5 \\
\hline orf19.6967 & USO6 & ER to Golgi vesicle-mediated transport & 1.9 & 1.7 & 1.3 & 1.1 & 1.3 & 1.4 \\
\hline orf19.4286 & & & 1.9 & 2.9 & 0.3 & 0.4 & 2.3 & 3.8 \\
\hline orf19.3658 & & protein folding in endoplasmic reticulum & 1.9 & 1.7 & 1.2 & 1.4 & 2.0 & 0.7 \\
\hline orf19.3536 & & protein amino acid N-linked glycosylation & 1.9 & 1.5 & 2.1 & 4.1 & 2.0 & 1.0 \\
\hline orf19.3659 & & transcription from RNA polymerase II promoter & 1.9 & 1.9 & 1.4 & 1.7 & 2.0 & 2.0 \\
\hline orf19.7567 & & & 1.9 & 2.1 & 1.1 & 1.0 & 0.2 & 0.2 \\
\hline orf19.2137 & & translation & 1.9 & 2.3 & 0.6 & 0.8 & 0.9 & 2.3 \\
\hline orf19.4871 & ERO1 & protein thiol-disulfide exchange & 1.9 & 1.9 & 0.9 & 0.6 & 1.8 & 1.7 \\
\hline orf19.2821 & & & 1.9 & 2.6 & 1.2 & 2.0 & 1.3 & 1.7 \\
\hline orf19.1317 & OSH3 & filamentous growth & 1.9 & 1.9 & 2.6 & 2.9 & 4.3 & 3.4 \\
\hline orf19.6607 & & & 1.9 & 1.9 & 2.2 & 2.5 & 0.6 & 1.9 \\
\hline orf19.5921 & & transport & 1.9 & 1.7 & 2.7 & 2.8 & 5.7 & 4.0 \\
\hline orf19.5847 & & transcription from RNA polymerase III promoter & 1.9 & 2.7 & 1.4 & 2.4 & 4.6 & 2.5 \\
\hline orf19.6382 & & transport & 1.9 & 2.7 & 1.4 & 1.8 & 7.3 & 2.3 \\
\hline orf19.2039 & MSF1 & mitochondrial phenylalanyl-tRNA aminoacylation & 1.9 & 1.8 & 1.8 & 2.6 & 1.1 & 1.4 \\
\hline orf19.6165 & $K G D 1$ & 2-oxoglutarate metabolic process & 1.9 & 3.6 & 2.7 & 3.5 & 3.2 & 2.0 \\
\hline orf19.367 & CNH1 & cell morphogenesis & 1.9 & 2.1 & 1.4 & 1.5 & 3.1 & 2.0 \\
\hline
\end{tabular}


Table A-1. (Continued)

\begin{tabular}{|c|c|c|c|c|c|c|c|c|}
\hline \multirow[b]{3}{*}{$\begin{array}{c}\text { orf19 } \\
\text { Designation }\end{array}$} & \multirow[b]{3}{*}{$\begin{array}{l}\text { CGD } \\
\text { Name }\end{array}$} & \multirow[b]{3}{*}{ Process $^{\mathrm{a}}$} & \multicolumn{6}{|c|}{ Change in Fold Expression } \\
\hline & & & \multicolumn{2}{|c|}{$\begin{array}{c}\text { SC5314+FLC } \\
\text { /SC5314 } \\
\end{array}$} & \multicolumn{2}{|c|}{$\begin{array}{c}\operatorname{cas} 5 \Delta / \Delta+\mathrm{FLC} \\
/ \operatorname{cas} 5 \Delta / \Delta\end{array}$} & \multicolumn{2}{|c|}{$\begin{array}{c}u p c 2 \Delta / \Delta+\mathrm{FLC} / \\
u p c 2 \Delta / \Delta\end{array}$} \\
\hline & & & Exp1 & Exp2 & Exp1 & Exp2 & Exp1 & Exp2 \\
\hline orf19.1687 & & maturation of 5.8S rRNA from tricistronic rRNA transcript & 1.9 & 2.3 & 2.6 & 4.7 & 3.5 & 2.3 \\
\hline orf19.2609 & CET1 & mRNA capping & 1.9 & 2.0 & 1.4 & 1.7 & 1.1 & 1.5 \\
\hline orf19.2064 & & regulation of transcription, DNA-dependent & 1.9 & 3.0 & 0.9 & 0.8 & 3.3 & 3.0 \\
\hline orf19.1252 & $Y M E 1$ & chronological cell aging & 1.9 & 3.0 & 1.0 & 0.7 & 1.1 & 1.3 \\
\hline orf19.3131 & OYE32 & cell redox homeostasis & 1.9 & 1.7 & 3.4 & 2.8 & 2.3 & 1.7 \\
\hline orf19.3881 & & & 1.9 & 2.0 & 0.7 & 0.6 & 0.5 & 0.5 \\
\hline orf19.6346 & & protein export from nucleus & 1.9 & 1.5 & 1.6 & 1.6 & 0.8 & 2.1 \\
\hline orf19.719 & & fungal-type cell wall organization & 1.8 & 3.3 & 1.0 & 1.0 & 1.5 & 1.3 \\
\hline orf19.4119 & SPO72 & autophagic vacuole formation & 1.8 & 2.0 & 1.7 & 1.3 & 4.2 & 3.1 \\
\hline orf19.782 & & lipid homeostasis & 1.8 & 1.7 & 2.1 & 2.6 & 4.9 & 2.7 \\
\hline orf19.7590 & & ATP synthesis coupled electron transport & 1.8 & 2.8 & 2.0 & 3.6 & 1.2 & 0.8 \\
\hline orf19.518 & & tRNA methylation & 1.8 & 1.9 & 1.3 & 2.1 & 1.1 & 1.0 \\
\hline orf19.6418 & & ribosomal large subunit biogenesis & 1.8 & 2.3 & 1.1 & 1.6 & 3.2 & 4.4 \\
\hline orf19.2256 & & & 1.8 & 1.5 & 2.1 & 2.8 & 1.5 & 3.4 \\
\hline orf19.4569 & & & 1.8 & 2.0 & 0.8 & 0.8 & 2.1 & 2.5 \\
\hline orf19.4330 & & & 1.8 & 1.9 & 2.0 & 2.3 & 1.3 & 1.8 \\
\hline orf19.5848 & & & 1.8 & 2.1 & 1.8 & 2.4 & 7.1 & 4.3 \\
\hline orf19.3290 & & electron transport chain & 1.8 & 2.0 & 1.8 & 2.3 & 1.0 & 1.1 \\
\hline orf19.1144 & & & 1.8 & 2.0 & 1.8 & 1.3 & 2.2 & 1.6 \\
\hline orf19.828 & & translation & 1.8 & 1.5 & 1.8 & 2.1 & 0.2 & 0.4 \\
\hline orf19.4393 & CIT1 & acetyl-CoA catabolic process & 1.8 & 1.9 & 4.2 & 6.6 & 2.3 & 1.1 \\
\hline orf19.913.2 & & aerobic respiration & 1.8 & 1.9 & 1.5 & 2.4 & 0.7 & 1.6 \\
\hline orf19.2057 & & protein complex assembly & 1.8 & 2.9 & 1.7 & 1.7 & 1.8 & 1.4 \\
\hline orf19.2590 & & biotin biosynthetic process & 1.8 & 2.8 & 1.6 & 1.4 & 0.0 & 0.2 \\
\hline orf19.5232 & CSI2 & filamentous growth & 1.8 & 1.7 & 1.3 & 2.6 & 1.3 & 2.0 \\
\hline orf19.3759 & & regulation of transcription from RNA polymerase II promoter & 1.8 & 1.5 & 1.7 & 3.3 & 4.4 & 3.6 \\
\hline orf19.2812 & & & 1.8 & 1.8 & 0.8 & 1.0 & 1.6 & 1.4 \\
\hline
\end{tabular}


Table A-1. (Continued)

\begin{tabular}{|c|c|c|c|c|c|c|c|c|}
\hline \multirow[b]{3}{*}{$\begin{array}{c}\text { orf19 } \\
\text { Designation }\end{array}$} & \multirow[b]{3}{*}{$\begin{array}{l}\text { CGD } \\
\text { Name }\end{array}$} & \multirow[b]{3}{*}{ Process $^{\mathrm{a}}$} & \multicolumn{6}{|c|}{ Change in Fold Expression } \\
\hline & & & \multicolumn{2}{|c|}{$\begin{array}{c}\text { SC5314+FLC } \\
\text { /SC5314 }\end{array}$} & \multicolumn{2}{|c|}{$\begin{array}{l}\operatorname{cas} 5 \Delta / \Delta+F L C \\
/ \operatorname{cas} 5 \Delta / \Delta\end{array}$} & \multicolumn{2}{|c|}{$\begin{array}{c}u p c 2 \Delta / \Delta+\mathrm{FLC} / \\
u p c 2 \Delta / \Delta\end{array}$} \\
\hline & & & Exp1 & Exp2 & Exp1 & Exp2 & Exp1 & Exp2 \\
\hline orf19.2044 & PGA27 & & 1.8 & 2.0 & 1.5 & 2.2 & 3.1 & 1.6 \\
\hline orf19.1954 & PUS4 & tRNA modification & 1.8 & 2.0 & 2.0 & 2.3 & 2.4 & 2.6 \\
\hline orf19.3573 & PEX6 & protein import into peroxisome matrix & 1.8 & 1.7 & 2.9 & 2.5 & 2.6 & 1.3 \\
\hline orf19.3310 & & & 1.8 & 1.9 & 0.8 & 0.6 & 2.9 & 3.3 \\
\hline orf19.2674 & & regulation of transcription, DNA-dependent & 1.8 & 20.1 & 1.1 & 1.2 & 1.2 & 1.5 \\
\hline orf19.5798 & LIG4 & filamentous growth & 1.8 & 2.6 & 0.9 & 0.8 & 1.0 & 1.0 \\
\hline orf19.6319 & & protein deubiquitination & 1.8 & 2.1 & 2.0 & 2.0 & 1.1 & 0.9 \\
\hline orf19.2565 & & mitochondrion inheritance & 1.8 & 2.6 & 1.3 & 1.4 & 5.4 & 4.3 \\
\hline orf19.5629 & QCR7 & aerobic respiration & 1.8 & 2.0 & 1.4 & 2.3 & 0.9 & 1.6 \\
\hline orf19.881 & $D F G 16$ & filamentous growth & 1.8 & 2.1 & 1.2 & 1.2 & 2.5 & 2.2 \\
\hline orf19.199 & & & 1.8 & 1.8 & 3.8 & 5.2 & 8.8 & 3.2 \\
\hline orf19.1888 & & ER-associated protein catabolic process & 1.8 & 1.8 & 0.9 & 1.0 & 1.6 & 2.3 \\
\hline orf19.4372 & & transmembrane transport & 1.8 & 3.0 & 0.6 & 0.6 & 3.5 & 3.3 \\
\hline orf19.409 & & mitochondrial respiratory chain complex IV assembly & 1.8 & 1.8 & 1.5 & 2.1 & 2.1 & 2.1 \\
\hline orf19.4175 & TOK1 & cellular potassium ion homeostasis & 1.8 & 3.4 & 2.3 & 2.5 & 4.3 & 4.8 \\
\hline orf19.6353 & & & 1.8 & 2.0 & 0.7 & 0.6 & 2.0 & 2.6 \\
\hline orf19.4488 & & ATP-dependent chromatin remodeling & 1.8 & 2.0 & 2.1 & 2.6 & 0.9 & 1.4 \\
\hline orf19.1574 & & RNA polymerase II transcriptional preinitiation complex assembly & 1.8 & 1.8 & 2.9 & 2.6 & 2.1 & 2.4 \\
\hline orf19.1631 & ERG6 & ergosterol biosynthetic process & 1.8 & 1.7 & 3.5 & 4.9 & 0.4 & 0.5 \\
\hline orf19.2516 & & protein folding & 1.8 & 1.7 & 3.3 & 3.5 & 1.5 & 2.0 \\
\hline orf19.6736 & & maturation of SSU-rRNA & 1.8 & 1.5 & 1.6 & 2.1 & 0.5 & 1.1 \\
\hline orf19.1864 & & & 1.8 & 1.5 & 1.3 & 1.2 & 1.5 & 2.1 \\
\hline orf19.4539 & & small GTPase mediated signal transduction & 1.8 & 1.7 & 0.9 & 0.5 & 1.5 & 2.3 \\
\hline orf19.2009 & $P E X 12$ & protein import into peroxisome matrix & 1.8 & 2.1 & 2.4 & 3.0 & 3.0 & 3.1 \\
\hline orf19.3065 & $D A O 1$ & oxidation-reduction process & 1.8 & 2.0 & 3.6 & 3.0 & 3.0 & 3.2 \\
\hline orf19.6047 & $T U F 1$ & mitochondrial translation & 1.8 & 2.8 & 1.5 & 1.4 & 0.3 & 0.3 \\
\hline orf19.874 & & misfolded or incompletely synthesized protein catabolic process & 1.8 & 1.5 & 1.1 & 1.0 & 0.5 & 1.0 \\
\hline
\end{tabular}


Table A-1. (Continued)

\begin{tabular}{|c|c|c|c|c|c|c|c|c|}
\hline \multirow{3}{*}{$\begin{array}{c}\text { orf19 } \\
\text { Designation }\end{array}$} & \multirow{3}{*}{$\begin{array}{l}\text { CGD } \\
\text { Name }\end{array}$} & \multirow[b]{3}{*}{ Process $^{\mathrm{a}}$} & \multicolumn{6}{|c|}{ Change in Fold Expression } \\
\hline & & & \multicolumn{2}{|c|}{$\begin{array}{c}\text { SC5314+FLC } \\
\text { /SC5314 } \\
\end{array}$} & \multicolumn{2}{|c|}{$\begin{array}{c}\operatorname{cas} 5 \Delta / \Delta+\mathrm{FLC} \\
/ \operatorname{cas} 5 \Delta / \Delta\end{array}$} & \multicolumn{2}{|c|}{$\begin{array}{c}u p c 2 \Delta / \Delta+\mathrm{FLC} \\
u p c 2 \Delta / \Delta\end{array}$} \\
\hline & & & Exp1 & Exp2 & Exp1 & Exp2 & Exp1 & Exp2 \\
\hline orf19.5159 & DUG3 & glutathione catabolic process & 1.8 & 3.6 & 0.9 & 0.7 & 1.3 & 1.3 \\
\hline orf19.5412 & & cell wall organization & 1.8 & 1.6 & 2.3 & 2.5 & 4.2 & 3.7 \\
\hline orf19.6152 & & & 1.8 & 1.6 & 3.6 & 3.8 & 3.0 & 2.4 \\
\hline orf19.6530 & & SRP-dependent cotranslational protein targeting to membrane & 1.8 & 2.4 & 1.7 & 2.9 & 6.6 & 4.0 \\
\hline orf19.7561 & $D E F 1$ & telomere maintenance & 1.8 & 4.8 & 1.3 & 2.5 & 2.4 & 2.7 \\
\hline orf19.6209 & & monocarboxylic acid transport & 1.8 & 1.6 & 2.0 & 2.0 & 2.6 & 1.6 \\
\hline orf19.5095 & & sterol transport & 1.8 & 2.2 & 1.8 & 2.1 & 2.4 & 1.4 \\
\hline orf19.4885 & MIRI & phosphate transport & 1.8 & 2.7 & 1.4 & 2.1 & 1.9 & 1.3 \\
\hline orf19.2808 & ZCF16 & & 1.8 & 2.5 & 1.9 & 2.4 & 4.8 & 3.4 \\
\hline orf19.4051 & HTS1 & histidyl-tRNA aminoacylation & 1.8 & 1.7 & 2.2 & 2.9 & 0.9 & 0.8 \\
\hline orf19.3811 & $G Y P 1$ & vesicle-mediated transport & 1.8 & 1.6 & 0.9 & 1.2 & 0.8 & 0.9 \\
\hline orf19.5356 & & fungal-type cell wall organization & 1.8 & 2.9 & 1.5 & 2.7 & 12.5 & 3.9 \\
\hline orf19.351 & & & 1.8 & 2.7 & 1.6 & 1.8 & 8.7 & 6.0 \\
\hline orf19.5392 & $N G T 1$ & $\mathrm{~N}$-acetylglucosamine transport & 1.8 & 2.0 & 1.6 & 1.6 & 44.3 & 29.7 \\
\hline orf19.5806 & ALD5 & acetate biosynthetic process & 1.8 & 1.7 & 2.7 & 2.3 & 2.0 & 1.0 \\
\hline orf19.6558 & & ER to Golgi vesicle-mediated transport & 1.8 & 1.8 & 1.8 & 2.8 & 2.6 & 2.3 \\
\hline orf19.75 & SEC5 & protein complex assembly & 1.8 & 2.0 & 3.2 & 1.8 & 2.1 & 1.6 \\
\hline orf19.5238 & & metabolic process & 1.8 & 2.5 & 1.8 & 1.9 & 2.8 & 4.2 \\
\hline orf19.4396 & & cristae formation & 1.8 & 1.9 & 1.8 & 2.3 & 1.0 & 0.9 \\
\hline orf19.2646 & ZCF 13 & aerobic respiration & 1.8 & 3.0 & 2.3 & 3.6 & 3.2 & 2.8 \\
\hline orf19.2753 & ZCF15 & regulation of transcription, DNA-dependent & 1.8 & 2.1 & 2.3 & 3.7 & 5.9 & 3.4 \\
\hline orf19.607 & & transcription-coupled nucleotide-excision repair & 1.8 & 2.0 & 2.1 & 1.8 & 2.5 & 2.5 \\
\hline orf19.4054 & CTA24 & positive regulation of transcription, DNA-dependent & 1.8 & 2.5 & 2.0 & 2.0 & 0.5 & 1.0 \\
\hline orf19.516 & & cell cycle & 1.8 & 2.0 & 1.2 & 1.7 & 1.1 & 1.0 \\
\hline orf19.920 & RMT2 & arginine metabolic process & 1.8 & 1.6 & 1.3 & 1.1 & 1.8 & 2.7 \\
\hline orf19.173 & & regulation of transcription, DNA-dependent & 1.8 & 4.2 & 1.3 & 2.0 & 2.3 & 1.6 \\
\hline orf19.7534 & MIS12 & folic acid-containing compound biosynthetic process & 1.8 & 5.2 & 1.3 & 2.5 & 3.2 & 1.7 \\
\hline
\end{tabular}


Table A-1. (Continued)

\begin{tabular}{|c|c|c|c|c|c|c|c|c|}
\hline \multirow[b]{3}{*}{$\begin{array}{c}\text { orf19 } \\
\text { Designation }\end{array}$} & \multirow[b]{3}{*}{$\begin{array}{l}\text { CGD } \\
\text { Name }\end{array}$} & \multirow[b]{3}{*}{ Process $^{\mathrm{a}}$} & \multicolumn{6}{|c|}{$\begin{array}{l}\text { Change in Fold Expression } \\
\end{array}$} \\
\hline & & & \multicolumn{2}{|c|}{$\begin{array}{c}\text { SC5314+FLC } \\
\text { /SC5314 } \\
\end{array}$} & \multicolumn{2}{|c|}{$\begin{array}{c}\operatorname{cas} 5 \Delta / \Delta+F L C \\
\quad / \operatorname{cas} 5 \Delta / \Delta \\
\end{array}$} & \multicolumn{2}{|c|}{$\begin{array}{c}u p c 2 \Delta / \Delta+\mathrm{FLC} / \\
u p c 2 \Delta / \Delta \\
\end{array}$} \\
\hline & & & Exp1 & Exp2 & Exp1 & Exp2 & Exp1 & Exp2 \\
\hline orf19.4066 & & phospholipid biosynthetic process & 1.8 & 1.6 & 2.2 & 2.2 & 1.7 & 0.7 \\
\hline orf19.5662 & PEP7 & filamentous growth & 1.8 & 1.6 & 1.0 & 0.7 & 3.1 & 4.8 \\
\hline orf19.7173 & & & 1.8 & 2.6 & 1.3 & 0.8 & 3.8 & 3.3 \\
\hline orf19.2699 & $A B P 1$ & actin cortical patch assembly & 1.8 & 1.9 & 5.2 & 3.8 & 2.4 & 1.1 \\
\hline orf19.255 & $Z C F 1$ & regulation of transcription, DNA-dependent & 1.8 & 5.7 & 3.3 & 4.7 & 9.2 & 2.3 \\
\hline orf19.209 & DFG10 & pseudohyphal growth & 1.8 & 1.5 & 1.4 & 1.4 & 3.3 & 2.4 \\
\hline orf19.4275 & RAD9 & hyphal growth & 1.8 & 1.5 & 1.1 & 2.7 & 1.1 & 1.4 \\
\hline orf19.6639 & & mitochondrial fission & 1.8 & 1.5 & 2.6 & 3.3 & 2.1 & 1.8 \\
\hline orf19.1957 & CYC3 & hyphal growth & 1.8 & 2.4 & 1.6 & 2.8 & 2.0 & 2.5 \\
\hline orf19.4862.2 & PET100 & aerobic respiration & 1.7 & 1.8 & 0.8 & 0.9 & 0.4 & 1.4 \\
\hline orf19.212 & VPS28 & fungal-type cell wall organization & 1.7 & 2.1 & 0.5 & 0.5 & 1.0 & 1.3 \\
\hline orf19.27 & & & 1.7 & 1.5 & 1.5 & 1.5 & 2.4 & 3.4 \\
\hline orf19.4758 & & oxidation-reduction process & 1.7 & 2.8 & 2.3 & 2.9 & 1.5 & 1.3 \\
\hline orf19.6211 & & chromosome segregation & 1.7 & 3.1 & 1.2 & 1.2 & 2.1 & 1.2 \\
\hline orf19.6698 & & mitochondrial asparaginyl-tRNA aminoacylation & 1.7 & 1.6 & 1.4 & 1.5 & 0.7 & 0.9 \\
\hline orf19.1434 & & nucleotide-excision repair & 1.7 & 2.7 & 0.8 & 1.0 & 0.8 & 0.9 \\
\hline orf19.1356 & & wobble position uridine thiolation & 1.7 & 2.1 & 1.8 & 3.2 & 3.1 & 2.4 \\
\hline orf19.1753 & PUS7 & enzyme-directed rRNA pseudouridine synthesis & 1.7 & 2.7 & 1.6 & 3.1 & 2.4 & 2.3 \\
\hline orf19.4511 & & & 1.7 & 1.8 & 1.5 & 1.7 & 2.8 & 1.6 \\
\hline orf19.2143 & & negative regulation of transposition, RNA-mediated & 1.7 & 2.4 & 1.4 & 2.8 & 8.2 & 3.5 \\
\hline orf19.4614 & & peroxisome degradation & 1.7 & 1.8 & 1.6 & 1.5 & 3.8 & 3.3 \\
\hline orf19.3962 & HAS1 & ribosomal small subunit biogenesis & 1.7 & 2.3 & 0.7 & 0.8 & 1.1 & 2.4 \\
\hline orf19.6445 & ECI1 & filamentous growth & 1.7 & 1.5 & 3.3 & 3.0 & 2.5 & 4.0 \\
\hline orf19.5940 & ZCF32 & lysine biosynthetic process via aminoadipic acid & 1.7 & 1.6 & 1.5 & 2.2 & 2.1 & 1.7 \\
\hline orf19.322 & & & 1.7 & 2.2 & 2.1 & 1.4 & 7.0 & 1.1 \\
\hline orf19.4864 & & triglyceride metabolic process & 1.7 & 1.6 & 1.8 & 1.6 & 8.7 & 6.4 \\
\hline orf19.5236 & & protein amino acid glycosylation & 1.7 & 2.2 & 0.5 & 0.4 & 1.0 & 1.8 \\
\hline
\end{tabular}


Table A-1. (Continued)

\begin{tabular}{|c|c|c|c|c|c|c|c|c|}
\hline \multirow[b]{3}{*}{$\begin{array}{c}\text { orf19 } \\
\text { Designation }\end{array}$} & \multirow[b]{3}{*}{$\begin{array}{l}\text { CGD } \\
\text { Name }\end{array}$} & \multirow[b]{3}{*}{ Process $^{\mathrm{a}}$} & \multicolumn{6}{|c|}{ Change in Fold Expression } \\
\hline & & & \multicolumn{2}{|c|}{$\begin{array}{c}\text { SC5314+FLC } \\
\text { /SC5314 } \\
\end{array}$} & \multicolumn{2}{|c|}{$\begin{array}{c}\operatorname{cas} 5 \Delta / \Delta+\mathrm{FLC} \\
/ \operatorname{cas} 5 \Delta / \Delta\end{array}$} & \multicolumn{2}{|c|}{$\begin{array}{c}u p c 2 \Delta / \Delta+\mathrm{FLC} \\
u p c 2 \Delta / \Delta\end{array}$} \\
\hline & & & Exp1 & $\operatorname{Exp2}$ & Exp1 & Exp2 & Exp1 & Exp2 \\
\hline orf19.254 & & & 1.7 & 2.6 & 2.3 & 2.6 & 2.9 & 1.7 \\
\hline orf19.4760 & & response to drug & 1.7 & 1.5 & 2.8 & 4.2 & 1.5 & 2.9 \\
\hline orf19.7114 & $C S A 1$ & cellular iron ion homeostasis & 1.7 & 3.2 & 1.5 & 1.5 & 0.7 & 0.6 \\
\hline orf19.5384 & CHS 8 & cell wall chitin biosynthetic process & 1.7 & 1.8 & 1.7 & 1.9 & 4.2 & 2.3 \\
\hline orf19.7544 & $T L O 1$ & & 1.7 & 1.6 & 2.2 & 1.7 & 0.9 & 1.3 \\
\hline orf19.1795 & PUF3 & aerobic respiration & 1.7 & 2.1 & 1.6 & 1.8 & 1.5 & 1.1 \\
\hline orf19.3586 & & fungal-type cell wall organization & 1.7 & 1.9 & 3.4 & 3.5 & 7.5 & 3.2 \\
\hline orf19.3342 & & protein deubiquitination & 1.7 & 2.0 & 1.1 & 0.9 & 2.4 & 2.5 \\
\hline orf19.3605 & $P E X 17$ & & 1.7 & 1.8 & 1.8 & 1.5 & 4.7 & 3.0 \\
\hline orf19.2387 & & prolyl-tRNA aminoacylation & 1.7 & 1.5 & 1.4 & 2.0 & 0.7 & 0.7 \\
\hline orf19.2908 & & oxidation-reduction process & 1.7 & 1.5 & 1.1 & 0.8 & 1.1 & 1.3 \\
\hline orf19.3221 & $C P A 2$ & arginine biosynthetic process & 1.7 & 3.9 & 2.7 & 3.5 & 5.2 & 2.2 \\
\hline orf19.3237 & & protein monoubiquitination & 1.7 & 2.1 & 1.4 & 1.4 & 1.8 & 2.1 \\
\hline orf19.847 & YIM1 & mitochondrial protein processing during import & 1.7 & 1.9 & 1.7 & 1.6 & 2.5 & 1.2 \\
\hline orf19.843 & & protein amino acid dephosphorylation & 1.7 & 1.7 & 1.2 & 1.9 & 2.6 & 1.2 \\
\hline orf19.211 & & DNA repair & 1.7 & 2.4 & 0.7 & 0.7 & 1.5 & 2.4 \\
\hline orf19.7092 & & & 1.7 & 1.5 & 1.4 & 1.5 & 1.0 & 1.0 \\
\hline orf19.5311 & & & 1.7 & 3.2 & 2.3 & 1.9 & 3.3 & 1.2 \\
\hline orf19.117 & & Golgi to plasma membrane transport & 1.7 & 1.5 & 2.5 & 2.5 & 3.9 & 3.6 \\
\hline orf19.1801 & $C B R 1$ & oxidation-reduction process & 1.7 & 1.5 & 1.3 & 1.7 & 1.6 & 1.3 \\
\hline orf19.7374 & CTA4 & positive regulation of transcription from RNA pol II promoter & 1.7 & 2.2 & 0.8 & 1.1 & 0.8 & 1.0 \\
\hline orf19.2248 & $A R E 2$ & ergosterol metabolic process & 1.7 & 1.6 & 0.7 & 0.7 & 1.1 & 1.2 \\
\hline orf19.6544 & LPI9 & chromosome segregation & 1.7 & 2.3 & 1.9 & 1.9 & 2.0 & 1.5 \\
\hline orf19.7527 & & & 1.7 & 2.1 & 1.0 & 1.0 & 2.3 & 1.9 \\
\hline orf19.3689 & & fructose metabolic process & 1.7 & 2.7 & 2.2 & 2.6 & 4.6 & 3.0 \\
\hline orf19.2791 & $B B C 1$ & actin cytoskeleton organization & 1.7 & 2.4 & 3.1 & 2.8 & 2.9 & 1.8 \\
\hline orf19.3171 & $A C H 1$ & acetate metabolic process & 1.7 & 2.0 & 1.9 & 2.4 & 3.9 & 0.9 \\
\hline
\end{tabular}


Table A-1. (Continued)

\begin{tabular}{|c|c|c|c|c|c|c|c|c|}
\hline \multirow{3}{*}{$\begin{array}{c}\text { orf19 } \\
\text { Designation }\end{array}$} & \multirow{3}{*}{$\begin{array}{l}\text { CGD } \\
\text { Name }\end{array}$} & \multirow[b]{3}{*}{ Process $^{\mathrm{a}}$} & \multicolumn{6}{|c|}{ Change in Fold Expression } \\
\hline & & & \multicolumn{2}{|c|}{$\begin{array}{c}\text { SC5314+FLC } \\
\text { /SC5314 }\end{array}$} & \multicolumn{2}{|c|}{$\begin{array}{l}\operatorname{cas} 5 \Delta / \Delta+F L C \\
/ \operatorname{cas} 5 \Delta / \Delta\end{array}$} & \multicolumn{2}{|c|}{$\begin{array}{c}u p c 2 \Delta / \Delta+\mathrm{FLC} / \\
u p c 2 \Delta / \Delta\end{array}$} \\
\hline & & & Exp1 & Exp2 & Exp1 & Exp2 & Exp1 & Exp2 \\
\hline orf19.3757 & ATP20 & ATP synthesis coupled proton transport & 1.7 & 1.9 & 1.2 & 1.5 & 1.1 & 1.1 \\
\hline orf19.273 & & sterol biosynthetic process & 1.7 & 1.5 & 1.3 & 1.7 & 2.2 & 1.4 \\
\hline orf19.2665 & MSN5 & protein export from nucleus & 1.7 & 2.5 & 1.2 & 1.0 & 2.3 & 1.5 \\
\hline orf19.3029 & & endocytosis & 1.7 & 1.8 & 3.4 & 3.0 & 2.6 & 2.7 \\
\hline orf19.5636 & RBT5 & cellular iron ion homeostasis & 1.7 & 1.6 & 1.8 & 1.5 & 0.2 & 0.2 \\
\hline orf19.3233 & HSE 1 & ascospore-type prospore assembly & 1.7 & 1.5 & 1.9 & 2.1 & 4.9 & 3.9 \\
\hline orf19.2077 & $Z C F 9$ & arginine metabolic process & 1.7 & 1.6 & 1.1 & 0.9 & 3.1 & 3.2 \\
\hline orf19.6479 & SEC1 & vesicle docking during exocytosis & 1.7 & 1.7 & 1.9 & 2.0 & 1.6 & 1.6 \\
\hline orf19.23 & RTA3 & fatty acid transport & 1.7 & 2.7 & 4.8 & 5.4 & 2.6 & 1.1 \\
\hline orf19.5912 & $M A K 21$ & ribosomal large subunit assembly & 1.7 & 3.5 & 1.3 & 2.1 & 2.5 & 3.1 \\
\hline orf19.6741 & & fungal-type cell wall organization & 1.7 & 1.7 & 1.7 & 2.5 & 0.9 & 1.1 \\
\hline orf19.1191 & & ER-associated protein catabolic process & 1.7 & 2.7 & 1.6 & 1.9 & 7.0 & 2.3 \\
\hline orf19.3026 & MAS1 & mitochondrial protein processing during import & 1.7 & 1.6 & 1.6 & 1.5 & 0.9 & 0.8 \\
\hline orf19.6187 & & & 1.7 & 1.7 & 2.1 & 3.0 & 0.9 & 0.7 \\
\hline orf19.5854.1 & & bile acid and bile salt transport & 1.7 & 2.2 & 1.4 & 2.8 & 2.0 & 4.5 \\
\hline orf19.4357 & & misfolded or incompletely synthesized protein catabolic process & 1.7 & 1.8 & 0.9 & 0.8 & 0.6 & 1.3 \\
\hline orf19.1210 & & amino acid transmembrane export from vacuole & 1.7 & 2.7 & 1.5 & 1.2 & 2.2 & 1.2 \\
\hline orf19.540 & & cellular protein modification process & 1.7 & 1.7 & 0.9 & 0.6 & 2.7 & 2.3 \\
\hline orf19.6794 & FESUR1 & oxidation-reduction process & 1.7 & 2.1 & 1.6 & 2.1 & 2.0 & 1.5 \\
\hline orf19.7160 & & response to osmotic stress & 1.7 & 1.9 & 1.1 & 1.5 & 1.9 & 3.3 \\
\hline orf19.6522 & & anion transport & 1.7 & 2.2 & 1.5 & 1.4 & 5.4 & 3.3 \\
\hline orf19.2242 & PRB1 & proteolysis & 1.7 & 1.5 & 0.7 & 0.6 & 2.5 & 2.5 \\
\hline orf19.1940 & & ubiquinone biosynthetic process & 1.7 & 1.5 & 1.0 & 1.0 & 0.4 & 0.7 \\
\hline orf19.563 & $R R P 15$ & maturation of rRNA & 1.7 & 1.5 & 1.5 & 4.0 & 2.2 & 5.9 \\
\hline orf19.2479 & $U G A 4$ & gamma-aminobutyric acid transport & 1.7 & 2.9 & 2.6 & 6.6 & 18.2 & 9.3 \\
\hline orf19.1622 & YCG1 & meiotic chromosome condensation & 1.7 & 2.1 & 2.3 & 3.9 & 1.3 & 1.7 \\
\hline orf19.6347 & & DNA replication & 1.7 & 1.5 & 1.7 & 2.8 & 1.0 & 1.9 \\
\hline
\end{tabular}


Table A-1. (Continued)

\begin{tabular}{|c|c|c|c|c|c|c|c|c|}
\hline \multirow[b]{3}{*}{$\begin{array}{c}\text { orf19 } \\
\text { Designation }\end{array}$} & \multirow[b]{3}{*}{$\begin{array}{l}\text { CGD } \\
\text { Name }\end{array}$} & \multirow[b]{3}{*}{ Process $^{a}$} & \multicolumn{6}{|c|}{ Change in Fold Expression } \\
\hline & & & \multicolumn{2}{|c|}{$\begin{array}{c}\text { SC5314+FLC } \\
\text { /SC5314 } \\
\end{array}$} & \multicolumn{2}{|c|}{$\begin{array}{c}\operatorname{cas} 5 \Delta / \Delta+F L C \\
\quad / \operatorname{cas} 5 \Delta / \Delta \\
\end{array}$} & \multicolumn{2}{|c|}{$\begin{array}{c}u p c 2 \Delta / \Delta+\mathrm{FLC} / \\
u p c 2 \Delta / \Delta\end{array}$} \\
\hline & & & Exp1 & Exp2 & Exp1 & Exp2 & Exp1 & Exp2 \\
\hline orf19.7675 & & translation & 1.7 & 1.9 & 1.8 & 2.6 & 0.8 & 0.8 \\
\hline orf19.7490 & & transmembrane transport & 1.7 & 2.2 & 1.0 & 1.8 & 6.1 & 3.8 \\
\hline orf19.3565 & & & 1.7 & 1.7 & 2.5 & 2.1 & 1.6 & 1.5 \\
\hline orf19.5518 & & & 1.7 & 1.6 & 1.4 & 1.4 & 5.1 & 2.1 \\
\hline orf19.4950 & $A K R 1$ & endocytosis & 1.7 & 1.8 & 1.7 & 1.7 & 5.1 & 2.6 \\
\hline orf19.6726 & & RNA elongation from RNA polymerase II promoter & 1.7 & 1.8 & 2.4 & 3.0 & 2.9 & 2.7 \\
\hline orf19.723 & $B C R 1$ & filamentous growth & 1.7 & 2.1 & 3.3 & 3.3 & 6.3 & 2.8 \\
\hline orf19.111 & CAN2 & amino acid transmembrane transport & 1.7 & 13.0 & 0.7 & 1.5 & 1.2 & 3.5 \\
\hline orf19.4318 & MIG1 & carbon utilization & 1.7 & 2.1 & 0.7 & 1.0 & 2.1 & 2.3 \\
\hline orf19.4747 & HEM14 & heme biosynthetic process & 1.7 & 2.0 & 0.6 & 0.4 & 0.1 & 0.2 \\
\hline orf19.2346 & & & 1.7 & 2.1 & 2.0 & 2.0 & 4.8 & 2.3 \\
\hline orf19.3519 & $S U A 72$ & transcription initiation from RNA polymerase II promoter & 1.7 & 1.7 & 1.1 & 1.1 & 1.7 & 2.0 \\
\hline orf19.6990 & & response to stress & 1.7 & 1.6 & 1.1 & 0.9 & 1.5 & 1.6 \\
\hline orf19.1367 & $M T W 1$ & chromosome segregation & 1.7 & 1.5 & 1.1 & 1.0 & 0.9 & 2.2 \\
\hline orf19.164 & & & 1.7 & 2.5 & 1.2 & 0.9 & 4.6 & 5.1 \\
\hline orf19.5884 & & rRNA metabolic process & 1.7 & 2.8 & 1.3 & 2.2 & 4.8 & 2.9 \\
\hline orf19.3133 & GUT2 & NADH oxidation & 1.7 & 1.8 & 1.1 & 1.1 & 2.2 & 1.6 \\
\hline orf19.4721 & & mRNA processing & 1.7 & 1.5 & 1.0 & 1.0 & 0.7 & 1.9 \\
\hline orf19.7379 & $F A A 2$ & lipid metabolic process & 1.7 & 3.1 & 1.3 & 1.2 & 4.4 & 2.8 \\
\hline orf19.1785 & & & 1.7 & 2.2 & 1.4 & 1.2 & 2.9 & 2.1 \\
\hline orf19.2734 & & oxidation-reduction process & 1.6 & 1.6 & 1.9 & 2.3 & 5.6 & 7.4 \\
\hline orf19.135 & EXO84 & Golgi to plasma membrane transport & 1.6 & 2.2 & 1.9 & 2.6 & 2.8 & 2.0 \\
\hline orf19.69 & & & 1.6 & 2.3 & 1.3 & 1.4 & 0.8 & 1.4 \\
\hline orf19.5250 & & & 1.6 & 1.5 & 3.8 & 3.2 & 4.3 & 4.5 \\
\hline orf19.7529 & EPL1 & histone acetylation & 1.6 & 2.5 & 1.0 & 1.1 & 2.0 & 1.5 \\
\hline orf19.2839 & CIRT4B & regulation of transcription, DNA-dependent & 1.6 & 2.6 & 0.4 & 0.3 & 0.7 & 0.6 \\
\hline orf19.1586 & $F G R 22$ & filamentous growth & 1.6 & 1.7 & 3.1 & 4.1 & 4.0 & 1.6 \\
\hline
\end{tabular}


Table A-1. (Continued)

\begin{tabular}{|c|c|c|c|c|c|c|c|c|}
\hline \multirow[b]{3}{*}{$\begin{array}{c}\text { orf19 } \\
\text { Designation }\end{array}$} & \multirow[b]{3}{*}{$\begin{array}{l}\text { CGD } \\
\text { Name }\end{array}$} & \multirow[b]{3}{*}{ Process $^{a}$} & \multicolumn{6}{|c|}{$\begin{array}{l}\text { Change in Fold Expression } \\
\end{array}$} \\
\hline & & & \multicolumn{2}{|c|}{$\begin{array}{c}\text { SC5314+FLC } \\
\text { /SC5314 } \\
\end{array}$} & \multicolumn{2}{|c|}{$\begin{array}{c}\operatorname{cas} 5 \Delta / \Delta+F L C \\
\quad / \operatorname{cas} 5 \Delta / \Delta \\
\end{array}$} & \multicolumn{2}{|c|}{$\begin{array}{c}u p c 2 \Delta / \Delta+\mathrm{FLC} / \\
u p c 2 \Delta / \Delta \\
\end{array}$} \\
\hline & & & Exp1 & Exp2 & Exp1 & Exp2 & Exp1 & Exp2 \\
\hline orf19.4640 & PWP1 & rRNA processing & 1.6 & 2.3 & 1.2 & 1.5 & 2.2 & 1.6 \\
\hline orf19.5419 & ATP5 & ATP synthesis coupled proton transport & 1.6 & 1.9 & 1.3 & 1.6 & 1.3 & 1.3 \\
\hline orf19.1255 & $Z C F 5$ & carbohydrate metabolic process & 1.6 & 2.0 & 2.2 & 1.7 & 6.4 & 4.2 \\
\hline orf19.5029 & $M O D F$ & & 1.6 & 2.1 & 1.3 & 1.3 & 2.0 & 1.5 \\
\hline orf19.285 & & mRNA stabilization & 1.6 & 1.5 & 1.0 & 1.0 & 1.5 & 2.8 \\
\hline orf19.4788 & $A R G 5,6$ & arginine biosynthetic process & 1.6 & 2.8 & 2.8 & 3.1 & 4.0 & 2.9 \\
\hline orf19.2439.1 & & aerobic respiration & 1.6 & 2.6 & 1.0 & 1.2 & 0.8 & 1.2 \\
\hline orf19.5543 & & Golgi to endosome transport & 1.6 & 1.5 & 3.6 & 3.1 & 10.1 & 5.3 \\
\hline orf19.1124 & & & 1.6 & 2.0 & 7.9 & 6.7 & 7.8 & 4.6 \\
\hline orf19.2031 & VPS 24 & protein targeting to vacuole & 1.6 & 1.9 & 2.5 & 3.2 & 6.1 & 6.5 \\
\hline orf19.5537 & WSC2 & cellular cell wall organization & 1.6 & 2.0 & 3.8 & 5.0 & 12.1 & 5.6 \\
\hline orf19.6652 & $D B P 8$ & endonucleolytic cleavage of rRNA & 1.6 & 2.5 & 1.9 & 3.0 & 3.8 & 4.1 \\
\hline orf19.6105 & $M V D$ & ergosterol biosynthetic process & 1.6 & 1.9 & 1.6 & 1.4 & 1.5 & 0.9 \\
\hline orf19.7629 & & & 1.6 & 1.8 & 1.7 & 1.7 & 3.5 & 2.1 \\
\hline orf19.5660 & & protein ubiquitination & 1.6 & 1.5 & 1.9 & 1.6 & 2.6 & 2.7 \\
\hline orf19.1698 & & actin cytoskeleton organization & 1.6 & 1.9 & 1.5 & 1.8 & 2.1 & 0.8 \\
\hline orf19.4739 & MSS116 & RNA splicing & 1.6 & 1.6 & 1.5 & 2.5 & 1.2 & 1.5 \\
\hline orf19.1467 & $C O X 13$ & aerobic respiration & 1.6 & 1.7 & 1.0 & 1.2 & 0.6 & 1.1 \\
\hline orf19.5953 & & regulation of cell size & 1.6 & 2.4 & 0.9 & 1.5 & 1.2 & 1.5 \\
\hline orf19.5705 & $N A M 2$ & mitochondrial translation & 1.6 & 2.0 & 1.3 & 1.9 & 0.7 & 0.7 \\
\hline orf19.6797 & & & 1.6 & 2.1 & 1.7 & 1.8 & 2.6 & 1.4 \\
\hline orf19.4906 & & $\mathrm{G} 1 / \mathrm{S}$ transition of mitotic cell cycle & 1.6 & 1.7 & 1.1 & 1.6 & 4.6 & 2.9 \\
\hline orf19.3706 & & oxidation-reduction process & 1.6 & 1.5 & 1.4 & 1.5 & 3.3 & 2.1 \\
\hline orf19.7365 & & anti-apoptosis & 1.6 & 1.5 & 1.5 & 1.6 & 1.9 & 1.7 \\
\hline orf19.1133 & $M S B 1$ & cell adhesion & 1.6 & 1.9 & 3.6 & 4.5 & 2.7 & 1.6 \\
\hline orf19.7521 & $R E P 1$ & response to drug & 1.6 & 3.7 & 2.2 & 7.2 & 4.5 & 1.3 \\
\hline orf19.3223 & ATP3 & ATP synthesis coupled proton transport & 1.6 & 2.1 & 1.2 & 1.3 & 1.5 & 1.5 \\
\hline
\end{tabular}


Table A-1. (Continued)

\begin{tabular}{|c|c|c|c|c|c|c|c|c|}
\hline \multirow[b]{3}{*}{$\begin{array}{c}\text { orf19 } \\
\text { Designation }\end{array}$} & \multirow[b]{3}{*}{$\begin{array}{c}\text { CGD } \\
\text { Name }\end{array}$} & \multirow[b]{3}{*}{ Process $^{a}$} & \multicolumn{6}{|c|}{ Change in Fold Expression } \\
\hline & & & \multicolumn{2}{|c|}{$\begin{array}{c}\text { SC5314+FLC } \\
\text { /SC5314 } \\
\end{array}$} & \multicolumn{2}{|c|}{$\begin{array}{c}\operatorname{cas} 5 \Delta / \Delta+\mathrm{FLC} \\
/ \operatorname{cas} 5 \Delta / \Delta \\
\end{array}$} & \multicolumn{2}{|c|}{$\begin{array}{c}u p c 2 \Delta / \Delta+\mathrm{FLC} / \\
u p c 2 \Delta / \Delta\end{array}$} \\
\hline & & & Exp1 & Exp2 & Exp1 & Exp2 & Exp1 & Exp2 \\
\hline orf19.166 & $A S G 1$ & positive regulation of transcription from RNA pol II promoter & 1.6 & 3.8 & 1.4 & 2.5 & 1.5 & 1.5 \\
\hline orf19.2104 & & iron-sulfur cluster assembly & 1.6 & 1.6 & 2.5 & 3.1 & 2.1 & 2.0 \\
\hline orf19.1945 & AUR1 & sphingolipid metabolic process & 1.6 & 2.3 & 1.3 & 2.1 & 7.1 & 3.8 \\
\hline orf19.6481 & YPS7 & response to pheromone during conjugation with cellular fusion & 1.6 & 2.3 & 0.4 & 0.4 & 0.9 & 1.0 \\
\hline orf19.1223 & $D B F 2$ & cytokinesis & 1.6 & 1.9 & 2.4 & 2.6 & 0.9 & 0.9 \\
\hline orf19.5247 & & & 1.6 & 1.5 & 1.0 & 1.4 & 1.5 & 1.7 \\
\hline orf19.5767 & & nuclear mRNA splicing, via spliceosome & 1.6 & 1.6 & 1.1 & 1.1 & 2.3 & 2.6 \\
\hline orf19.922 & ERG11 & ergosterol biosynthetic process & 1.6 & 1.5 & 1.2 & 1.0 & 0.8 & 0.7 \\
\hline orf19.2881 & MNN4 & cell wall mannoprotein biosynthetic process & 1.6 & 2.4 & 1.2 & 1.6 & 2.3 & 1.3 \\
\hline orf19.5535 & & anion transport & 1.6 & 1.5 & 1.6 & 2.1 & 1.6 & 1.3 \\
\hline orf19.4159 & & transport & 1.6 & 1.5 & 3.0 & 4.7 & 1.2 & 1.0 \\
\hline orf19.2314 & & rRNA processing & 1.6 & 1.5 & 2.7 & 4.2 & 1.5 & 7.5 \\
\hline orf19.5296 & & mitochondrial respiratory chain complex IV assembly & 1.6 & 1.6 & 1.1 & 1.3 & 0.7 & 1.3 \\
\hline orf19.5335 & SGS1 & double-strand break repair via homologous recombination & 1.6 & 1.9 & 1.4 & 2.4 & 2.9 & 1.3 \\
\hline orf19.4478 & & mitochondrial aspartyl-tRNA aminoacylation & 1.6 & 1.8 & 1.0 & 0.9 & 0.5 & 0.8 \\
\hline orf19.879 & & intracellular mRNA localization & 1.6 & 2.2 & 1.4 & 1.1 & 2.5 & 2.6 \\
\hline orf19.59 & REII & budding cell bud growth & 1.6 & 1.8 & 2.6 & 4.4 & 1.0 & 4.6 \\
\hline orf19.5139 & & & 1.6 & 1.8 & 1.2 & 1.4 & 3.2 & 1.4 \\
\hline orf19.6973 & & proteolysis & 1.6 & 2.1 & 2.2 & 2.3 & 2.2 & 1.9 \\
\hline orf19.2296 & & & 1.6 & 2.2 & 2.6 & 3.0 & 1.7 & 0.8 \\
\hline orf19.3616 & $E R G 9$ & ergosterol biosynthetic process & 1.6 & 1.7 & 1.2 & 1.0 & 0.9 & 1.1 \\
\hline orf19.2343 & VPS23 & hyphal growth & 1.6 & 1.8 & 2.5 & 1.7 & 2.8 & 2.5 \\
\hline orf19.6196 & & chromosome segregation & 1.6 & 1.5 & 2.7 & 2.8 & 2.3 & 3.2 \\
\hline orf19.4363 & $S G D 1$ & hyperosmotic response & 1.6 & 1.7 & 1.9 & 3.7 & 2.1 & 2.8 \\
\hline orf19.4504 & & fermentation & 1.6 & 2.7 & 3.7 & 3.1 & 4.5 & 6.1 \\
\hline orf19.399 & & sphingolipid metabolic process & 1.6 & 2.0 & 1.2 & 1.1 & 3.8 & 2.4 \\
\hline orf19.7519 & & & 1.6 & 1.9 & 1.1 & 1.1 & 1.4 & 1.6 \\
\hline
\end{tabular}


Table A-1. (Continued)

\begin{tabular}{|c|c|c|c|c|c|c|c|c|}
\hline \multirow[b]{3}{*}{$\begin{array}{c}\text { orf19 } \\
\text { Designation }\end{array}$} & \multirow[b]{3}{*}{$\begin{array}{l}\text { CGD } \\
\text { Name }\end{array}$} & \multirow[b]{3}{*}{ Process $^{a}$} & \multicolumn{6}{|c|}{ Change in Fold Expression } \\
\hline & & & \multicolumn{2}{|c|}{$\begin{array}{c}\text { SC5314+FLC } \\
\text { /SC5314 } \\
\end{array}$} & \multicolumn{2}{|c|}{$\begin{array}{c}\operatorname{cas} 5 \Delta / \Delta+\mathrm{FLC} \\
/ \operatorname{cas} 5 \Delta / \Delta\end{array}$} & \multicolumn{2}{|c|}{$\begin{array}{c}u p c 2 \Delta / \Delta+\mathrm{FLC} / \\
u p c 2 \Delta / \Delta\end{array}$} \\
\hline & & & Exp1 & Exp2 & Exp1 & Exp2 & Exp1 & Exp2 \\
\hline orf19.1297 & & early endosome to Golgi transport & 1.6 & 2.7 & 5.7 & 4.1 & 6.3 & 10.2 \\
\hline orf19.2663 & & & 1.6 & 1.9 & 1.0 & 1.2 & 1.2 & 0.9 \\
\hline orf19.2670 & & fatty acid biosynthetic process & 1.6 & 1.8 & 0.4 & 0.4 & 0.4 & 0.7 \\
\hline orf19.5058 & SMI1 & cellular bud neck & 1.6 & 2.1 & 1.6 & 1.3 & 0.7 & 1.2 \\
\hline orf19.781 & DUR31 & urea transport & 1.6 & 1.6 & 2.0 & 3.0 & 6.4 & 3.3 \\
\hline orf19.3311 & IFD3 & cellular aldehyde metabolic process & 1.6 & 1.6 & 2.1 & 2.2 & 2.7 & 3.3 \\
\hline orf19.3991 & & cellular lipid metabolic process & 1.6 & 1.5 & 1.4 & 1.7 & 2.5 & 1.9 \\
\hline orf19.2410 & SYS3 & Golgi to plasma membrane protein transport & 1.6 & 1.9 & 3.8 & 3.1 & 2.0 & 2.7 \\
\hline orf19.5539 & & retrograde vesicle-mediated transport, Golgi to ER & 1.6 & 1.6 & 0.7 & 0.8 & 1.6 & 2.9 \\
\hline orf19.886 & PAN1 & axial cellular bud site selection & 1.6 & 1.9 & 2.4 & 4.4 & 1.7 & 1.1 \\
\hline orf19.4688 & $D A G 7$ & cellular response to drug & 1.6 & 1.6 & 2.3 & 2.6 & 3.5 & 1.7 \\
\hline orf19.1464 & BMT5 & glycosphingolipid biosynthetic process & 1.6 & 2.1 & 1.5 & 1.5 & 2.5 & 1.3 \\
\hline orf19.3360 & & & 1.6 & 2.0 & 0.9 & 1.0 & 1.8 & 2.1 \\
\hline orf19.2068 & & & 1.6 & 1.5 & 1.3 & 1.8 & 0.6 & 0.6 \\
\hline orf19.3508 & & nicotinamide nucleotide metabolic process & 1.6 & 1.9 & 1.8 & 1.7 & 1.7 & 1.2 \\
\hline orf19.4896 & & transcription from RNA polymerase I promoter & 1.6 & 2.0 & 2.2 & 3.5 & 1.3 & 2.5 \\
\hline orf19.6550 & & & 1.6 & 1.6 & 1.0 & 1.0 & 1.5 & 1.7 \\
\hline orf19.3535 & & & 1.6 & 1.8 & 1.4 & 2.2 & 8.0 & 5.5 \\
\hline orf19.6898.1 & & & 1.6 & 1.6 & 1.1 & 1.5 & 0.6 & 0.9 \\
\hline orf19.7599 & UTP5 & maturation of rRNA & 1.6 & 1.5 & 1.3 & 2.6 & 2.1 & 1.7 \\
\hline orf19.7270 & & & 1.6 & 2.1 & 1.6 & 1.4 & 2.2 & 1.0 \\
\hline orf19.5892 & & protein monoubiquitination & 1.6 & 2.7 & 1.3 & 1.0 & 3.1 & 2.4 \\
\hline orf19.6045 & & phosphatidylcholine biosynthetic process & 1.6 & 1.9 & 0.9 & 1.0 & 1.9 & 1.6 \\
\hline orf19.5110 & OPY2 & cell cycle arrest in response to pheromone & 1.6 & 2.3 & 2.9 & 3.5 & 13.6 & 4.9 \\
\hline orf19.4771 & & protein amino acid glycosylation & 1.6 & 1.8 & 1.2 & 1.3 & 6.5 & 8.1 \\
\hline orf19.7350 & $R C T 1$ & & 1.6 & 1.6 & 1.6 & 1.7 & 1.4 & 1.1 \\
\hline orf19.5989 & & mRNA cleavage & 1.6 & 2.0 & 2.0 & 2.0 & 1.7 & 1.4 \\
\hline
\end{tabular}


Table A-1. (Continued)

\begin{tabular}{|c|c|c|c|c|c|c|c|c|}
\hline \multirow[b]{3}{*}{$\begin{array}{c}\text { orf19 } \\
\text { Designation }\end{array}$} & \multirow[b]{3}{*}{$\begin{array}{l}\text { CGD } \\
\text { Name }\end{array}$} & \multirow[b]{3}{*}{ Process $^{\mathrm{a}}$} & \multicolumn{6}{|c|}{ Change in Fold Expression } \\
\hline & & & \multicolumn{2}{|c|}{$\begin{array}{c}\text { SC5314+FLC } \\
\text { /SC5314 } \\
\end{array}$} & \multicolumn{2}{|c|}{$\begin{array}{c}\operatorname{cas} 5 \Delta / \Delta+F L C \\
\quad / c a s 5 \Delta / \Delta \\
\end{array}$} & \multicolumn{2}{|c|}{$\begin{array}{c}\text { upc } 2 \Delta / \Delta+\mathrm{FLC} / \\
u p c 2 \Delta / \Delta\end{array}$} \\
\hline & & & Exp1 & Exp2 & Exp1 & Exp2 & Exp1 & Exp2 \\
\hline orf19.522 & & cellular chaperone-mediated protein complex assembly & 1.6 & 1.7 & 2.0 & 2.2 & 1.1 & 1.1 \\
\hline orf19.2736 & HFL2 & negative regulation of transcription from RNA pol II promoter & 1.6 & 2.1 & 1.1 & 1.3 & 1.6 & 1.4 \\
\hline orf19.6217 & $P G A 63$ & ER to Golgi vesicle-mediated transport & 1.6 & 2.8 & 2.5 & 3.3 & 1.6 & 0.6 \\
\hline orf19.260 & SLD1 & plasma membrane organization & 1.6 & 1.5 & 1.3 & 1.5 & 1.0 & 1.4 \\
\hline orf19.1881 & & glycerophospholipid biosynthetic process & 1.6 & 2.1 & 1.1 & 1.4 & 1.9 & 1.3 \\
\hline orf19.1648 & RAD50 & response to oxidative stress & 1.6 & 1.7 & 2.2 & 1.7 & 1.8 & 1.4 \\
\hline orf19.5407 & SOF1 & maturation of rRNA & 1.6 & 1.6 & 1.4 & 2.2 & 2.2 & 3.8 \\
\hline orf19.2644 & QCR2 & aerobic respiration & 1.6 & 2.4 & 1.4 & 1.6 & 1.3 & 1.1 \\
\hline orf19.3579 & ATP4 & ATP synthesis coupled proton transport & 1.6 & 1.8 & 1.4 & 2.0 & 0.9 & 1.4 \\
\hline orf19.418 & KOG1 & regulation of cell growth & 1.6 & 2.0 & 1.4 & 1.6 & 3.0 & 1.4 \\
\hline orf19.6942 & ORC3 & DNA replication initiation & 1.6 & 1.7 & 1.9 & 2.5 & 1.5 & 1.4 \\
\hline orf19.4670 & CAS5 & fungal-type cell wall organization & 1.6 & 2.8 & 0.1 & 0.3 & 4.7 & 3.3 \\
\hline orf19.6643 & MCT1 & fatty acid metabolic process & 1.6 & 1.5 & 3.0 & 3.0 & 2.6 & 1.6 \\
\hline orf19.5893 & $R I P 1$ & aerobic respiration & 1.6 & 2.2 & 1.4 & 1.9 & 0.7 & 0.8 \\
\hline orf19.3122 & $A R R 3$ & arsenite transport & 1.6 & 1.9 & 1.6 & 1.3 & 3.3 & 3.2 \\
\hline orf19.5005 & OSM2 & metabolic process & 1.6 & 1.5 & 2.6 & 3.6 & 2.6 & 1.9 \\
\hline orf19.3553 & $R P F 2$ & ribosomal large subunit assembly & 1.6 & 1.8 & 1.1 & 2.2 & 1.0 & 1.5 \\
\hline orf19.603 & $I M P 4$ & translation & 1.5 & 1.6 & 1.5 & 3.2 & 2.4 & 3.5 \\
\hline orf19.7481 & $M D H 1$ & tricarboxylic acid cycle & 1.5 & 1.6 & 1.4 & 1.4 & 1.9 & 1.0 \\
\hline orf19.109 & & mitochondrial tyrosyl-tRNA aminoacylation & 1.5 & 1.6 & 1.3 & 1.2 & 0.5 & 0.5 \\
\hline orf19.1374 & & & 1.5 & 3.1 & 1.3 & 1.4 & 3.8 & 4.9 \\
\hline orf19.3138 & NOP1 & rRNA methylation & 1.5 & 2.4 & 1.2 & 1.5 & 0.7 & 0.7 \\
\hline orf19.1301 & & & 1.5 & 2.0 & 3.8 & 3.2 & 1.1 & 2.0 \\
\hline orf19.5500 & $M A K 16$ & maturation of rRNA & 1.5 & 1.9 & 3.5 & 6.1 & 0.9 & 6.1 \\
\hline orf19.5357 & $A K L 1$ & actin cortical patch assembly & 1.5 & 1.8 & 1.9 & 1.9 & 3.4 & 2.1 \\
\hline orf19.1515 & CHT4 & chitin catabolic process & 1.5 & 1.5 & 1.0 & 0.7 & 5.5 & 4.8 \\
\hline orf19.5797 & $P I-P L C$ & filamentous growth & 1.5 & 2.0 & 2.7 & 3.9 & 4.1 & 1.5 \\
\hline
\end{tabular}


Table A-1. (Continued)

\begin{tabular}{|c|c|c|c|c|c|c|c|c|}
\hline \multirow[b]{3}{*}{$\begin{array}{c}\text { orf19 } \\
\text { Designation }\end{array}$} & \multirow[b]{3}{*}{$\begin{array}{c}\text { CGD } \\
\text { Name }\end{array}$} & \multirow[b]{3}{*}{ Process $^{\mathrm{a}}$} & \multicolumn{6}{|c|}{ Change in Fold Expression } \\
\hline & & & \multicolumn{2}{|c|}{$\begin{array}{c}\text { SC5314+FLC } \\
\text { /SC5314 } \\
\end{array}$} & \multicolumn{2}{|c|}{$\begin{array}{c}\operatorname{cas} 5 \Delta / \Delta+\mathrm{FLC} \\
/ \operatorname{cas} 5 \Delta / \Delta\end{array}$} & \multicolumn{2}{|c|}{$\begin{array}{c}u p c 2 \Delta / \Delta+\mathrm{FLC} \\
u p c 2 \Delta / \Delta\end{array}$} \\
\hline & & & Exp1 & $\operatorname{Exp2}$ & Exp1 & Exp2 & Exp1 & Exp2 \\
\hline orf19.1589 & & regulation of transcription, DNA-dependent & 1.5 & 2.3 & 1.5 & 3.2 & 4.7 & 4.9 \\
\hline orf19.7225 & & & 1.5 & 1.5 & 1.7 & 2.1 & 1.7 & 3.6 \\
\hline orf19.4750 & & & 1.5 & 1.8 & 2.3 & 4.2 & 2.4 & 1.1 \\
\hline orf19.5326 & & glucose metabolic process & 1.5 & 1.9 & 0.6 & 0.7 & 1.3 & 3.4 \\
\hline orf19.6531 & NUC2 & oxidation-reduction process & 1.5 & 2.0 & 1.7 & 2.2 & 1.2 & 1.0 \\
\hline orf19.3546 & $P E X 2$ & protein import into peroxisome matrix & 1.5 & 1.7 & 1.7 & 2.1 & 3.5 & 2.0 \\
\hline orf19.3841 & & autophagic vacuole formation & 1.5 & 2.6 & 2.2 & 2.8 & 9.1 & 5.3 \\
\hline orf19.4418 & FMT1 & methionyl-tRNA aminoacylation & 1.5 & 1.5 & 1.5 & 2.7 & 0.9 & 1.9 \\
\hline orf19.4106 & & & 1.5 & 2.1 & 3.2 & 4.6 & 4.7 & 3.5 \\
\hline orf19.2073 & & drug transmembrane transport & 1.5 & 1.6 & 0.6 & 0.5 & 2.1 & 2.8 \\
\hline orf19.633 & & metabolic process & 1.5 & 1.6 & 0.8 & 0.5 & 0.3 & 1.1 \\
\hline orf19.6464 & & & 1.5 & 1.8 & 1.0 & 1.2 & 3.3 & 2.4 \\
\hline orf19.7537 & BNR1 & cell separation during cytokinesis & 1.5 & 2.3 & 1.8 & 2.3 & 4.4 & 2.8 \\
\hline orf19.2046 & POT1-2 & fatty acid beta-oxidation & 1.5 & 2.2 & 0.5 & 0.4 & 1.6 & 1.9 \\
\hline orf19.5504 & & & 1.5 & 2.1 & 1.5 & 1.8 & 3.2 & 2.0 \\
\hline orf19.314 & & histone deacetylation & 1.5 & 1.8 & 1.4 & 1.7 & 1.9 & 1.5 \\
\hline orf19.6194 & & & 1.5 & 1.6 & 1.2 & 1.3 & 0.6 & 0.9 \\
\hline orf19.7357 & & coenzyme A biosynthetic process & 1.5 & 1.7 & 2.0 & 2.6 & 2.7 & 2.5 \\
\hline orf19.6117 & & transmembrane transport & 1.5 & 2.3 & 0.6 & 0.4 & 3.3 & 3.3 \\
\hline orf19.2786 & & vesicle-mediated transport & 1.5 & 2.2 & 1.4 & 1.7 & 1.6 & 1.6 \\
\hline orf19.1956 & & reciprocal meiotic recombination & 1.5 & 1.8 & 1.7 & 2.5 & 0.8 & 1.0 \\
\hline orf19.2789 & & & 1.5 & 1.8 & 1.3 & 1.3 & 1.6 & 1.7 \\
\hline orf19.6014 & RRS1 & response to drug & 1.5 & 1.7 & 1.3 & 2.3 & 2.7 & 4.2 \\
\hline orf19.5365 & & & 1.5 & 1.7 & 1.6 & 2.4 & 0.9 & 0.9 \\
\hline orf19.1658 & & & 1.5 & 1.7 & 2.5 & 3.5 & 3.3 & 2.1 \\
\hline orf19.6996 & & protein amino acid O-linked glycosylation & 1.5 & 2.9 & 1.0 & 2.5 & 1.4 & 2.1 \\
\hline orf19.7664 & & & 1.5 & 1.7 & 0.7 & 1.6 & 1.3 & 2.4 \\
\hline
\end{tabular}


Table A-1. (Continued)

\begin{tabular}{|c|c|c|c|c|c|c|c|c|}
\hline \multirow[b]{3}{*}{$\begin{array}{c}\text { orf19 } \\
\text { Designation }\end{array}$} & \multirow[b]{3}{*}{$\begin{array}{l}\text { CGD } \\
\text { Name }\end{array}$} & \multirow[b]{3}{*}{ Process $^{\mathrm{a}}$} & \multicolumn{6}{|c|}{ Change in Fold Expression } \\
\hline & & & \multicolumn{2}{|c|}{$\begin{array}{c}\text { SC5314+FLC } \\
\text { /SC5314 }\end{array}$} & \multicolumn{2}{|c|}{$\begin{array}{l}\operatorname{cas} 5 \Delta / \Delta+F L C \\
/ \operatorname{cas} 5 \Delta / \Delta\end{array}$} & \multicolumn{2}{|c|}{$\begin{array}{c}u p c 2 \Delta / \Delta+\mathrm{FLC} / \\
u p c 2 \Delta / \Delta\end{array}$} \\
\hline & & & Exp1 & Exp2 & Exp1 & Exp2 & Exp1 & Exp2 \\
\hline orf19.6837 & FMA1 & oxidation-reduction process & 1.5 & 1.5 & 0.7 & 0.7 & 0.9 & 1.6 \\
\hline orf19.2608 & ADH5 & biofilm formation & 1.5 & 1.7 & 1.7 & 1.5 & 1.2 & 1.2 \\
\hline orf19.2308 & & fructose 2,6-bisphosphate metabolic process & 1.5 & 1.6 & 0.7 & 1.0 & 1.4 & 1.9 \\
\hline orf19.729.1 & $R G D 3$ & intracellular signal transduction & 1.5 & 2.0 & 1.0 & 0.8 & 2.3 & 2.2 \\
\hline orf19.3969 & SFL2 & filamentous growth & 1.5 & 2.4 & 1.2 & 1.2 & 0.7 & 0.6 \\
\hline orf19.3782.2 & & cristae formation & 1.5 & 1.8 & 1.1 & 1.5 & 0.6 & 0.5 \\
\hline orf19.6140 & FRE30 & & 1.5 & 4.8 & 9.5 & 6.1 & 23.3 & 15.4 \\
\hline orf19.1047 & ERB1 & rRNA processing & 1.5 & 1.9 & 1.0 & 1.6 & 1.2 & 2.2 \\
\hline orf19.1373 & INP51 & fungal-type cell wall organization & 1.5 & 1.8 & 2.1 & 2.4 & 3.7 & 2.7 \\
\hline orf19.2691 & & & 1.5 & 1.5 & 0.5 & 0.7 & 0.4 & 0.7 \\
\hline orf19.6989 & & & 1.5 & 2.5 & 1.6 & 1.4 & 3.2 & 3.7 \\
\hline orf19.6621 & MHP1 & filamentous growth & 1.5 & 2.4 & 1.0 & 0.9 & 1.3 & 1.0 \\
\hline orf19.6527 & & conjugation with cellular fusion & 1.5 & 1.8 & 0.8 & 0.7 & 4.4 & 2.7 \\
\hline orf19.1325 & ECM38 & & 1.5 & 1.9 & 1.6 & 1.2 & 1.9 & 1.5 \\
\hline orf19.7509.1 & ATP17 & ATP synthesis coupled proton transport & 1.5 & 1.5 & 1.1 & 1.1 & 1.3 & 1.9 \\
\hline orf19.6601 & & & 1.5 & 3.0 & 4.1 & 7.2 & 6.2 & 8.4 \\
\hline orf19.649 & & & 1.5 & 2.2 & 1.2 & 1.5 & 3.0 & 2.3 \\
\hline orf19.6448 & & & 1.5 & 2.3 & 1.9 & 2.2 & 1.2 & 0.6 \\
\hline orf19.6512 & EXO70 & Golgi to plasma membrane transport & 1.5 & 1.8 & 2.6 & 2.4 & 2.0 & 1.7 \\
\hline orf19.916 & & apoptotic process & 1.5 & 1.7 & 1.8 & 1.8 & 3.7 & 2.3 \\
\hline orf19.4033 & PRP22 & generation of catalytic spliceosome for second transesterification step & 1.5 & 1.8 & 1.9 & 2.3 & 2.6 & 2.8 \\
\hline orf19.1563 & ECM3 & cell wall organization & 1.5 & 1.5 & 0.4 & 0.6 & 1.2 & 1.5 \\
\hline orf19.3461 & & & 1.5 & 1.6 & 2.2 & 2.9 & 1.7 & 1.1 \\
\hline orf19.1077 & ATM1 & cellular iron ion homeostasis & 1.5 & 2.8 & 4.5 & 2.9 & 3.2 & 3.4 \\
\hline orf19.4918 & & regulation of transcription, DNA-dependent & 1.5 & 1.7 & 1.7 & 1.9 & 2.4 & 1.5 \\
\hline orf19.4759 & COX5 & aerobic respiration & 1.5 & 2.2 & 1.5 & 1.6 & 0.9 & 1.2 \\
\hline orf19.3758 & & & 1.5 & 1.6 & 1.0 & 0.9 & 1.6 & 2.0 \\
\hline
\end{tabular}


Table A-1. (Continued)

\begin{tabular}{|c|c|c|c|c|c|c|c|c|}
\hline \multirow{3}{*}{$\begin{array}{c}\text { orf19 } \\
\text { Designation }\end{array}$} & \multirow{3}{*}{$\begin{array}{c}\text { CGD } \\
\text { Name }\end{array}$} & \multirow[b]{3}{*}{ Process $^{\mathrm{a}}$} & \multicolumn{6}{|c|}{$\begin{array}{l}\text { Change in Fold Expression } \\
\end{array}$} \\
\hline & & & \multicolumn{2}{|c|}{$\begin{array}{c}\text { SC5314+FLC } \\
\text { /SC5314 }\end{array}$} & \multicolumn{2}{|c|}{$\begin{array}{c}\operatorname{cas} 5 \Delta / \Delta+\mathrm{FLC} \\
/ \operatorname{cas} 5 \Delta / \Delta\end{array}$} & \multicolumn{2}{|c|}{$\begin{array}{c}u p c 2 \Delta / \Delta+\mathrm{FLC} / \\
u p c 2 \Delta / \Delta\end{array}$} \\
\hline & & & Exp1 & Exp2 & Exp1 & Exp2 & Exp1 & Exp2 \\
\hline orf19.5167 & IFM1 & mitochondrial translation & 1.5 & 1.7 & 1.9 & 3.2 & 0.8 & 1.4 \\
\hline orf19.7551 & ALO1 & response to oxidative stress & 1.5 & 2.8 & 0.7 & 0.6 & 1.7 & 1.3 \\
\hline orf19.6225 & $P C L 7$ & regulation of cyclin-dependent protein kinase activity & 1.5 & 2.0 & 0.8 & 0.8 & 1.5 & 1.3 \\
\hline orf19.5660.1 & & ATP synthesis coupled proton transport & 1.5 & 1.8 & 1.1 & 1.6 & 1.2 & 1.6 \\
\hline orf19.1816 & $A L S 3$ & biofilm formation & 1.5 & 3.1 & 1.3 & 1.3 & 2.6 & 1.1 \\
\hline orf19.1981 & $I M P 2$ & mitochondrial protein processing during import & 1.5 & 1.5 & 1.8 & 2.3 & 1.3 & 2.0 \\
\hline orf19.7619 & & mitochondrion organization & 1.5 & 1.9 & 2.1 & 2.6 & 3.6 & 3.5 \\
\hline orf19.191 & $\mathrm{KICl}$ & cell wall organization & 1.5 & 1.9 & 1.2 & 1.2 & 1.7 & 1.3 \\
\hline orf19.4634 & & protein urmylation & 1.5 & 3.2 & 2.1 & 2.4 & 3.2 & 3.8 \\
\hline orf19.7661 & HMII & mitochondrial genome maintenance & 1.5 & 1.6 & 1.4 & 1.0 & 2.0 & 1.9 \\
\hline orf19.2309 & & RNA processing & 1.5 & 1.6 & 1.8 & 4.0 & 1.2 & 1.3 \\
\hline orf19.6979 & & cellular manganese ion homeostasis & 1.5 & 5.7 & 3.8 & 2.5 & 5.3 & 2.9 \\
\hline orf19.3827 & & misfolded or incompletely synthesized protein catabolic process & 1.5 & 1.6 & 1.4 & 2.3 & 6.2 & 3.6 \\
\hline orf19.1371 & & & 1.5 & 1.6 & 1.3 & 1.7 & 0.8 & 0.6 \\
\hline orf19.1900 & & nucleolus organization & 1.5 & 1.6 & 2.4 & 3.8 & 1.1 & 1.5 \\
\hline orf19.7187 & MAM33 & aerobic respiration & 1.5 & 2.3 & 1.7 & 1.3 & 1.1 & 1.5 \\
\hline orf19.7027 & & & 1.5 & 2.2 & 0.7 & 1.3 & 2.4 & 2.2 \\
\hline orf19.4805 & & Golgi to plasma membrane transport & 1.5 & 1.5 & 1.3 & 2.5 & 2.5 & 1.3 \\
\hline orf19.4127 & & actin cortical patch localization & 1.5 & 1.8 & 1.8 & 1.8 & 1.6 & 1.2 \\
\hline orf19.783 & & intracellular protein transport & 1.5 & 2.2 & 2.0 & 3.4 & 7.4 & 3.2 \\
\hline orf19.3309 & & & 1.5 & 1.7 & 0.8 & 0.6 & 3.2 & 2.8 \\
\hline orf19.738 & MYO5 & cell wall organization & 1.5 & 2.0 & 1.9 & 1.9 & 2.7 & 1.4 \\
\hline orf19.4645 & BEM1 & cell budding & 1.5 & 1.9 & 1.5 & 1.6 & 0.4 & 0.6 \\
\hline orf19.3201 & MTLA1 & cell differentiation & 1.5 & 1.5 & 1.8 & 2.1 & 2.4 & 4.5 \\
\hline orf19.1671 & UTR2 & cell wall organization & 1.5 & 1.6 & 1.9 & 2.5 & 3.8 & 2.6 \\
\hline orf19.3302 & & regulation of glycogen biosynthetic process & 1.5 & 2.1 & 0.7 & 0.8 & 1.1 & 0.9 \\
\hline orf19.5619 & & & 1.5 & 2.9 & 1.4 & 1.6 & 5.7 & 3.8 \\
\hline
\end{tabular}


Table A-1. (Continued)

\begin{tabular}{|c|c|c|c|c|c|c|c|c|}
\hline \multirow{3}{*}{$\begin{array}{c}\text { orf19 } \\
\text { Designation }\end{array}$} & \multirow{3}{*}{$\begin{array}{c}\text { CGD } \\
\text { Name }\end{array}$} & \multirow[b]{3}{*}{ Process $^{\mathrm{a}}$} & \multicolumn{6}{|c|}{ Change in Fold Expression } \\
\hline & & & \multicolumn{2}{|c|}{$\begin{array}{l}\text { SC5314+FLC } \\
\text { /SC5314 } \\
\end{array}$} & \multicolumn{2}{|c|}{$\begin{array}{c}\operatorname{cas} 5 \Delta / \Delta+\mathrm{FLC} \\
/ \operatorname{cas} 5 \Delta / \Delta\end{array}$} & \multicolumn{2}{|c|}{$\begin{array}{c}u p c 2 \Delta / \Delta+\mathrm{FLC} \\
u p c 2 \Delta / \Delta\end{array}$} \\
\hline & & & Exp1 & $\operatorname{Exp2}$ & Exp1 & Exp2 & Exp1 & Exp2 \\
\hline orf19.6297 & & tRNA pseudouridine synthesis & 1.5 & 1.8 & 1.5 & 2.0 & 2.4 & 2.6 \\
\hline orf19.5134 & & & 1.5 & 2.2 & 0.9 & 2.0 & 1.7 & 6.0 \\
\hline orf19.2006.1 & $C O X 17$ & intracellular copper ion transport & 1.5 & 1.6 & 1.8 & 1.2 & 0.7 & 1.0 \\
\hline orf19.5234 & $R B D 1$ & filamentous growth & 1.5 & 1.8 & 1.0 & 1.2 & 4.3 & 2.7 \\
\hline orf19.5168 & & maturation of SSU-rRNA & 1.5 & 1.7 & 1.6 & 2.4 & 1.9 & 2.3 \\
\hline orf19.4764 & & mRNA 3'-end processing & 1.5 & 1.5 & 1.9 & 1.8 & 2.5 & 1.1 \\
\hline orf19.7569 & SIK1 & rRNA processing & 1.5 & 2.2 & 1.3 & 1.7 & 0.6 & 0.9 \\
\hline orf19.1776 & & coenzyme A biosynthetic process & 1.5 & 1.5 & 1.6 & 1.9 & 0.6 & 1.0 \\
\hline orf19.3083 & & DNA recombination & 1.5 & 1.8 & 1.0 & 1.6 & 0.8 & 0.3 \\
\hline orf19.4457 & BNI4 & filamentous growth & 1.5 & 2.0 & 1.4 & 1.9 & 2.2 & 1.0 \\
\hline orf19.3603 & & multicellular organismal development & 1.5 & 1.5 & 2.8 & 2.8 & 1.6 & 1.5 \\
\hline orf19.6070 & ENA2 & potassium ion transport & 1.5 & 2.1 & 0.9 & 1.7 & 11.7 & 6.1 \\
\hline orf19.6854 & $A T P 1$ & ATP biosynthetic process & 1.5 & 2.0 & 1.2 & 1.0 & 1.2 & 1.0 \\
\hline orf19.3195 & $H I P 1$ & amino acid transmembrane transport & 1.5 & 2.6 & 0.6 & 0.7 & 0.7 & 0.8 \\
\hline orf19.1187 & $\mathrm{CPH} 2$ & filamentous growth & 1.5 & 2.2 & 1.4 & 1.7 & 3.5 & 2.1 \\
\hline orf19.3004 & & & 1.5 & 2.0 & 0.6 & 0.6 & 0.9 & 1.4 \\
\hline orf19.4317 & GRE3 & response to oxidative stress & 1.5 & 1.5 & 0.8 & 0.6 & 0.3 & 0.7 \\
\hline orf19.2443 & $R G D 1$ & osmosensory signaling pathway & 1.5 & 1.6 & 1.4 & 1.2 & 1.6 & 1.7 \\
\hline
\end{tabular}

${ }^{\mathrm{a}}$ Descriptions are from the Candida Genome Database (http://www.candidagenome.org). 
Table A-2. Genes downregulated by at least 0.5-fold by fluconazole in SC5314

\begin{tabular}{|c|c|c|c|c|c|c|c|c|}
\hline \multirow{3}{*}{$\begin{array}{c}\text { orf19 } \\
\text { Designation }\end{array}$} & \multirow{3}{*}{$\begin{array}{c}\text { CGD } \\
\text { Name }\end{array}$} & \multirow[b]{3}{*}{ Process $^{a}$} & \multicolumn{6}{|c|}{ Change in Fold Expression } \\
\hline & & & \multicolumn{2}{|c|}{$\begin{array}{c}\text { SC5314+FLC } \\
\text { /SC5314 } \\
\end{array}$} & \multicolumn{2}{|c|}{$\begin{array}{c}\operatorname{cas} 5 \Delta / \Delta+\mathrm{FLC} \\
/ \operatorname{cas} 5 \Delta / \Delta\end{array}$} & \multicolumn{2}{|c|}{$\begin{array}{c}u p c 2 \Delta / \Delta+\mathrm{FLC} \\
u p c 2 \Delta / \Delta\end{array}$} \\
\hline & & & Exp1 & $\operatorname{Exp2}$ & Exp1 & $\operatorname{Exp2}$ & Exp1 & Exp2 \\
\hline orf19.5673 & OPT7 & oligopeptide transport & 0.0 & 0.1 & 0.2 & 0.2 & 0.0 & 0.0 \\
\hline orf19.2475 & $P G A 26$ & filamentous growth & 0.1 & 0.0 & 0.2 & 0.2 & 0.1 & 0.2 \\
\hline orf19.946 & MET14 & methionine metabolic process & 0.1 & 0.1 & 1.1 & 2.5 & 0.4 & 0.2 \\
\hline orf19.689 & PLB1 & pathogenesis & 0.1 & 0.0 & 0.0 & 0.0 & 0.0 & 0.0 \\
\hline orf19.2337 & $A L P 1$ & basic amino acid transport & 0.1 & 0.1 & 0.3 & 0.4 & 0.4 & 0.3 \\
\hline orf19.5017 & DUR32 & transmembrane transport & 0.1 & 0.1 & 0.7 & 0.6 & 0.9 & 0.2 \\
\hline orf19.3475 & & & 0.1 & 0.1 & 0.4 & 0.4 & 2.0 & 2.1 \\
\hline orf19.2738 & SUL2 & sulfate transport & 0.1 & 0.3 & 1.7 & 1.2 & 5.4 & 0.8 \\
\hline orf19.5399 & $I F F 11$ & pathogenesis & 0.1 & 0.1 & 0.0 & 0.0 & 0.0 & 0.0 \\
\hline orf19.1887 & & sterol metabolic process & 0.1 & 0.1 & 0.4 & 0.5 & 0.6 & 0.5 \\
\hline orf19.7330 & & thiamin biosynthetic process & 0.1 & 0.2 & 0.4 & 0.3 & 0.1 & 0.2 \\
\hline orf19.6679 & & & 0.1 & 0.3 & 1.0 & 2.8 & 1.0 & 1.5 \\
\hline orf19.386 & SAM4 & L-methionine biosynthetic process from S-adenosylmethionine & 0.2 & 0.1 & 0.3 & 0.3 & 0.1 & 0.1 \\
\hline orf19.1442 & PLB4.5 & phosphoinositide metabolic process & 0.2 & 0.3 & 0.4 & 0.3 & 0.0 & 0.0 \\
\hline orf19.769 & IFE1 & oxidation-reduction process & 0.2 & 0.1 & 1.0 & 1.2 & 0.1 & 0.1 \\
\hline orf19.4526 & HSP30 & negative regulation of ATPase activity & 0.2 & 0.1 & 2.6 & 1.2 & 0.8 & 1.5 \\
\hline orf19.4669 & AAT22 & aspartate catabolic process & 0.2 & 0.1 & 0.3 & 0.4 & 0.0 & 0.0 \\
\hline orf19.385 & $G C V 2$ & glycine catabolic process & 0.2 & 0.3 & 1.2 & 2.5 & 0.9 & 0.1 \\
\hline orf19.1368 & & & 0.2 & 0.3 & 0.2 & 0.1 & 0.1 & 0.1 \\
\hline orf19.2618 & MET2 & homoserine metabolic process & 0.2 & 0.2 & 0.8 & 0.9 & 0.6 & 0.3 \\
\hline orf19.5103 & & & 0.2 & 0.3 & 0.1 & 0.1 & 0.1 & 0.2 \\
\hline orf19.6770 & & actin cortical patch assembly & 0.2 & 0.2 & 0.1 & 0.1 & 0.2 & 0.2 \\
\hline orf19.6086 & LEU4 & leucine biosynthetic process & 0.2 & 0.3 & 0.6 & 0.8 & 0.1 & 0.1 \\
\hline orf19.7284 & ASR2 & & 0.2 & 0.1 & 1.7 & 1.7 & 0.3 & 0.3 \\
\hline orf19.7098 & & oxidation-reduction process & 0.2 & 0.1 & 0.9 & 0.8 & 0.6 & 0.9 \\
\hline orf19.1447 & & & 0.2 & 0.3 & 1.0 & 2.7 & 0.7 & 0.3 \\
\hline
\end{tabular}


Table A-2. (Continued)

\begin{tabular}{|c|c|c|c|c|c|c|c|c|}
\hline \multirow{3}{*}{$\begin{array}{c}\text { orf19 } \\
\text { Designation }\end{array}$} & \multirow{3}{*}{$\begin{array}{c}\text { CGD } \\
\text { Name }\end{array}$} & \multirow[b]{3}{*}{ Process $^{\mathrm{a}}$} & \multicolumn{6}{|c|}{ Change in Fold Expression } \\
\hline & & & \multicolumn{2}{|c|}{$\begin{array}{c}\text { SC5314+FLC } \\
\text { /SC5314 }\end{array}$} & \multicolumn{2}{|c|}{$\begin{array}{c}\operatorname{cas} 5 \Delta / \Delta+\mathrm{FLC} \\
/ \operatorname{cas} 5 \Delta / \Delta\end{array}$} & \multicolumn{2}{|c|}{$\begin{array}{c}u p c 2 \Delta / \Delta+\mathrm{FLC} / \\
u p c 2 \Delta / \Delta\end{array}$} \\
\hline & & & Exp1 & Exp2 & Exp1 & Exp2 & Exp1 & Exp2 \\
\hline orf19.768 & SYG1 & signal transduction & 0.2 & 0.4 & 0.1 & 0.1 & 0.4 & 0.6 \\
\hline orf19.5870 & CTP1 & mitochondrial citrate transport & 0.2 & 0.2 & 0.2 & 0.4 & 0.1 & 0.3 \\
\hline orf19.6570 & NUP & nucleoside transport & 0.2 & 0.1 & 0.7 & 0.3 & 0.1 & 0.1 \\
\hline orf19.7553 & & & 0.2 & 0.5 & 3.6 & 3.2 & 0.7 & 1.2 \\
\hline orf19.4664 & NAT4 & cellular developmental process & 0.2 & 0.2 & 0.1 & 0.1 & 0.0 & 0.1 \\
\hline orf19.7586 & CHT3 & cellular bud site selection & 0.2 & 0.3 & 0.2 & 0.2 & 0.0 & 0.0 \\
\hline orf19.3897 & & & 0.2 & 0.3 & 1.5 & 1.2 & 0.2 & 0.5 \\
\hline orf19.3916 & & & 0.2 & 0.2 & 0.9 & 0.6 & 0.4 & 0.5 \\
\hline orf19.3048 & & & 0.2 & 0.3 & 0.5 & 0.9 & 0.9 & 0.3 \\
\hline orf19.3106 & MET16 & methionine metabolic process & 0.2 & 0.3 & 0.8 & 1.4 & 1.2 & 0.6 \\
\hline orf19.4456 & GAP4 & amino acid transport & 0.2 & 0.3 & 0.5 & 0.7 & 0.9 & 0.6 \\
\hline orf19.923 & THR1 & homoserine metabolic process & 0.2 & 0.2 & 0.2 & 0.2 & 0.1 & 0.2 \\
\hline orf19.4438 & $R M E 1$ & meiosis & 0.2 & 0.4 & 0.4 & 0.3 & 0.0 & 0.2 \\
\hline orf19.6077 & & & 0.2 & 0.3 & 0.3 & 0.3 & 0.9 & 0.5 \\
\hline orf19.7199 & & & 0.3 & 0.2 & 0.1 & 0.2 & 0.1 & 0.2 \\
\hline orf19.4907 & & regulation of cell size & 0.3 & 0.4 & 0.5 & 0.4 & 0.3 & 0.2 \\
\hline orf19.4651 & $P G A 53$ & & 0.3 & 0.2 & 0.4 & 0.4 & 0.2 & 0.2 \\
\hline orf19.3664 & $H S P 31$ & protein refolding & 0.3 & 0.2 & 1.0 & 0.8 & 0.3 & 0.7 \\
\hline orf19.7310 & & reciprocal meiotic recombination & 0.3 & 0.3 & 2.5 & 2.6 & 1.1 & 0.5 \\
\hline orf19.5992 & WOR2 & cellular developmental process & 0.3 & 0.2 & 0.5 & 0.4 & 0.9 & 0.6 \\
\hline orf19.4889 & & drug transport & 0.3 & 0.2 & 0.7 & 1.0 & 0.6 & 0.5 \\
\hline orf19.7025 & $M C M 1$ & hyphal growth & 0.3 & 0.4 & 0.5 & 0.6 & 0.5 & 0.7 \\
\hline orf19.4142 & & & 0.3 & 0.3 & 1.5 & 6.2 & 2.7 & 1.1 \\
\hline orf19.2602 & OPT1 & nitrogen utilization & 0.3 & 0.2 & 0.8 & 0.5 & 0.1 & 0.1 \\
\hline orf19.1333 & SNG3 & response to drug & 0.3 & 0.3 & 0.2 & 0.2 & 0.5 & 0.4 \\
\hline orf19.4375 & & chromatin silencing at rDNA & 0.3 & 0.3 & 0.4 & 0.5 & 0.4 & 0.8 \\
\hline orf19.1075 & & & 0.3 & 0.5 & 1.5 & 1.4 & 2.5 & 1.4 \\
\hline
\end{tabular}


Table A-2. (Continued)

\begin{tabular}{|c|c|c|c|c|c|c|c|c|}
\hline \multirow[b]{3}{*}{$\begin{array}{c}\text { orf19 } \\
\text { Designation }\end{array}$} & \multirow[b]{3}{*}{$\begin{array}{l}\text { CGD } \\
\text { Name }\end{array}$} & \multirow[b]{3}{*}{$\begin{array}{r}\text { Process }^{\mathrm{a}} \\
\end{array}$} & \multicolumn{6}{|c|}{ Change in Fold Expression } \\
\hline & & & \multicolumn{2}{|c|}{$\begin{array}{c}\text { SC5314+FLC } \\
\text { /SC5314 } \\
\end{array}$} & \multicolumn{2}{|c|}{$\begin{array}{c}\operatorname{cas} 5 \Delta / \Delta+\mathrm{FLC} \\
/ \operatorname{cas} 5 \Delta / \Delta \\
\end{array}$} & \multicolumn{2}{|c|}{$\begin{array}{c}\text { upc } 2 \Delta / \Delta+\mathrm{FLC} / \\
\text { upc } 2 \Delta / \Delta\end{array}$} \\
\hline & & & Exp1 & Exp2 & Exp1 & Exp2 & Exp1 & Exp2 \\
\hline orf19.2018 & & peptidyl-diphthamide biosynthetic process & 0.3 & 0.3 & 2.1 & 4.1 & 2.0 & 2.3 \\
\hline orf19.5663 & $\mathrm{RCH1}$ & calcium ion import & 0.3 & 0.2 & 0.9 & 0.8 & 1.3 & 1.6 \\
\hline orf19.2246 & & & 0.3 & 0.1 & 0.6 & 0.8 & 0.7 & 2.1 \\
\hline orf19.3433 & OYE23 & oxidation-reduction process & 0.3 & 0.5 & 0.5 & 0.2 & 0.1 & 0.1 \\
\hline orf19.473 & TPO4 & drug transport & 0.3 & 0.3 & 0.9 & 1.0 & 0.5 & 0.4 \\
\hline orf19.5484 & SER1 & purine base biosynthetic process & 0.3 & 0.3 & 0.8 & 0.9 & 0.4 & 0.5 \\
\hline orf19.5615 & $A Y R 2$ & phosphatidic acid biosynthetic process & 0.3 & 0.2 & 0.3 & 0.2 & 0.2 & 0.2 \\
\hline orf19.21 & & ethanol metabolic process & 0.3 & 0.3 & 0.2 & 0.2 & 0.3 & 0.8 \\
\hline orf19.5805 & $D L D 1$ & aerobic respiration & 0.3 & 0.5 & 1.1 & 1.5 & 0.9 & 0.5 \\
\hline orf19.1510 & $S R D 1$ & & 0.3 & 0.4 & 0.8 & 1.4 & 1.8 & 0.8 \\
\hline orf19.1159 & & & 0.3 & 0.5 & 1.6 & 2.9 & 4.0 & 1.1 \\
\hline orf19.3915 & & glutathione catabolic process & 0.3 & 0.4 & 0.4 & 0.5 & 0.4 & 0.3 \\
\hline orf19.6656 & DUR3 & urea transport & 0.3 & 0.3 & 0.3 & 0.3 & 0.4 & 0.3 \\
\hline orf19.6636 & & & 0.3 & 0.2 & 0.4 & 0.4 & 0.2 & 0.2 \\
\hline orf19.1443 & PLB4 & phosphoinositide metabolic process & 0.3 & 0.4 & 0.5 & 0.4 & 0.1 & 0.0 \\
\hline orf19.1182 & & Golgi to vacuole transport & 0.3 & 0.3 & 0.7 & 1.0 & 0.8 & 0.5 \\
\hline orf19.1968.1 & & N-terminal protein amino acid acetylation & 0.3 & 0.3 & 1.1 & 1.6 & 0.4 & 0.3 \\
\hline orf19.5032 & SIM1 & cell wall organization & 0.3 & 0.4 & 0.6 & 1.0 & 0.8 & 1.0 \\
\hline orf19.1901 & $M C M 3$ & DNA replication initiation & 0.3 & 0.4 & 1.1 & 2.1 & 0.2 & 0.1 \\
\hline orf19.2396 & $I F R 2$ & oxidation-reduction process & 0.3 & 0.2 & 0.9 & 0.6 & 0.2 & 0.1 \\
\hline orf19.1253 & PHO4 & cellular response to phosphate starvation & 0.3 & 0.2 & 1.0 & 1.5 & 0.8 & 0.6 \\
\hline orf19.2639 & & mitochondrial genome maintenance & 0.3 & 0.2 & 1.0 & 2.5 & 0.1 & 0.6 \\
\hline orf19.4192 & $C D C 14$ & hyphal growth & 0.3 & 0.2 & 0.6 & 0.8 & 0.3 & 0.5 \\
\hline orf19.6817 & FCR1 & filamentous growth & 0.3 & 0.4 & 0.1 & 0.1 & 0.3 & 0.2 \\
\hline orf19.4733 & $Y M C 2$ & transport & 0.3 & 0.3 & 0.9 & 0.9 & 0.9 & 0.4 \\
\hline orf19.139 & TRA1 & histone acetylation & 0.3 & 0.4 & 2.3 & 1.1 & 0.7 & 0.4 \\
\hline orf19.6580 & & & 0.3 & 0.3 & 0.5 & 0.5 & 0.1 & 0.2 \\
\hline
\end{tabular}


Table A-2. (Continued)

\begin{tabular}{|c|c|c|c|c|c|c|c|c|}
\hline \multirow{3}{*}{$\begin{array}{c}\text { orf19 } \\
\text { Designation }\end{array}$} & \multirow{3}{*}{$\begin{array}{c}\text { CGD } \\
\text { Name }\end{array}$} & \multirow[b]{3}{*}{ Process $^{\mathrm{a}}$} & \multicolumn{6}{|c|}{ Change in Fold Expression } \\
\hline & & & \multicolumn{2}{|c|}{$\begin{array}{c}\text { SC5314+FLC } \\
\text { /SC5314 } \\
\end{array}$} & \multicolumn{2}{|c|}{$\begin{array}{c}\operatorname{cas} 5 \Delta / \Delta+\mathrm{FLC} \\
/ \operatorname{cas} 5 \Delta / \Delta\end{array}$} & \multicolumn{2}{|c|}{$\begin{array}{c}u p c 2 \Delta / \Delta+\mathrm{FLC} / \\
u p c 2 \Delta / \Delta\end{array}$} \\
\hline & & & Exp1 & Exp2 & Exp1 & Exp2 & Exp1 & Exp2 \\
\hline orf19.4816 & & & 0.3 & 0.2 & 0.4 & 0.3 & 0.5 & 0.6 \\
\hline orf19.1517 & ARO3 & aromatic amino acid family biosynthetic process & 0.3 & 0.3 & 0.7 & 1.1 & 0.2 & 0.2 \\
\hline orf19.5316 & FGR29 & filamentous growth & 0.3 & 0.5 & 0.9 & 0.9 & 0.7 & 0.5 \\
\hline orf19.5519 & $G C V 1$ & glycine metabolic process & 0.3 & 0.2 & 1.0 & 1.0 & 1.1 & 0.6 \\
\hline orf19.2452 & & & 0.3 & 0.2 & 0.2 & 0.5 & 0.2 & 0.1 \\
\hline orf19.1116 & & & 0.3 & 0.2 & 0.2 & 0.1 & 0.2 & 0.3 \\
\hline orf19.1390 & PMII & fungal-type cell wall organization & 0.3 & 0.3 & 0.4 & 0.4 & 0.3 & 0.2 \\
\hline orf19.5267 & & & 0.3 & 0.3 & 0.2 & 0.3 & 0.0 & 0.0 \\
\hline orf19.2989 & GORI & glyoxylate catabolic process & 0.3 & 0.3 & 0.5 & 0.4 & 0.2 & 0.2 \\
\hline orf19.1697 & & cytoplasmic translation & 0.3 & 0.4 & 1.0 & 1.9 & 0.5 & 0.9 \\
\hline orf19.2600 & SPC98 & microtubule nucleation & 0.4 & 0.4 & 1.6 & 3.7 & 1.7 & 1.3 \\
\hline orf19.655 & PHO84 & phosphate transport & 0.4 & 0.5 & 0.6 & 0.5 & 0.1 & 0.6 \\
\hline orf19.6001 & $S A P 3$ & adhesion to host & 0.4 & 0.2 & 0.1 & 0.1 & 0.1 & 1.1 \\
\hline orf19.5496 & $A V T 1$ & & 0.4 & 0.4 & 0.3 & 0.5 & 0.5 & 0.7 \\
\hline orf19.335 & & chromosome segregation & 0.4 & 0.3 & 0.6 & 0.6 & 0.7 & 0.8 \\
\hline orf19.1621 & $G P A 2$ & hyphal growth & 0.4 & 0.5 & 0.7 & 0.8 & 0.9 & 1.0 \\
\hline orf19.4718 & TRP5 & tryptophan biosynthetic process & 0.4 & 0.4 & 0.8 & 1.1 & 0.3 & 0.5 \\
\hline orf19.4068 & & & 0.4 & 0.4 & 1.1 & 1.1 & 0.5 & 0.4 \\
\hline orf19.6022 & NRMI & cellular response to hydroxyurea & 0.4 & 0.4 & 1.9 & 2.5 & 0.3 & 0.2 \\
\hline orf19.1258 & & & 0.4 & 0.2 & 0.9 & 1.2 & 1.0 & 0.4 \\
\hline orf19.1756 & GPD1 & glycerol metabolic process & 0.4 & 0.4 & 0.2 & 0.2 & 0.1 & 0.2 \\
\hline orf19.7218 & $R B E 1$ & pathogenesis & 0.4 & 0.3 & 0.2 & 0.4 & 0.0 & 0.0 \\
\hline orf19.3648 & & DNA repair & 0.4 & 0.3 & 1.0 & 1.2 & 0.4 & 0.4 \\
\hline orf19.7166 & & & 0.4 & 0.1 & 0.5 & 0.6 & 0.3 & 0.4 \\
\hline orf19.5051 & & & 0.4 & 0.2 & 1.3 & 2.3 & 0.6 & 0.9 \\
\hline orf19.3230 & BOI2 & budding cell apical bud growth & 0.4 & 0.4 & 1.0 & 1.4 & 1.5 & 0.9 \\
\hline orf19.6951 & & calcium-mediated signaling & 0.4 & 0.5 & 0.7 & 0.9 & 1.1 & 0.5 \\
\hline
\end{tabular}


Table A-2. (Continued)

\begin{tabular}{|c|c|c|c|c|c|c|c|c|}
\hline \multirow{3}{*}{$\begin{array}{c}\text { orf19 } \\
\text { Designation }\end{array}$} & \multirow{3}{*}{$\begin{array}{c}\text { CGD } \\
\text { Name }\end{array}$} & \multirow[b]{3}{*}{ Process $^{\mathrm{a}}$} & \multicolumn{6}{|c|}{ Change in Fold Expression } \\
\hline & & & \multicolumn{2}{|c|}{$\begin{array}{c}\text { SC5314+FLC } \\
\text { /SC5314 } \\
\end{array}$} & \multicolumn{2}{|c|}{$\begin{array}{c}\operatorname{cas} 5 \Delta / \Delta+F L C / \\
\operatorname{cas} 5 \Delta / \Delta\end{array}$} & \multicolumn{2}{|c|}{$\begin{array}{c}u p c 2 \Delta / \Delta+\mathrm{FLC} / \\
u p c 2 \Delta / \Delta\end{array}$} \\
\hline & & & Exp1 & Exp2 & Exp1 & Exp2 & Exp1 & Exp2 \\
\hline orf19.7190 & $O G G 1$ & base-excision repair, AP site formation & 0.4 & 0.4 & 3.5 & 5.1 & 1.6 & 0.6 \\
\hline orf19.2335 & & response to drug & 0.4 & 0.3 & 0.3 & 0.3 & 0.2 & 0.2 \\
\hline orf19.6782 & $B M T 1$ & beta-1,2-oligomannoside metabolic process & 0.4 & 0.4 & 1.0 & 0.8 & 0.1 & 0.1 \\
\hline orf19.5760 & IHDI & & 0.4 & 0.5 & 0.1 & 0.3 & 0.1 & 0.4 \\
\hline orf19.3160 & $H S P 12$ & cell adhesion & 0.4 & 0.3 & 1.7 & 1.4 & 0.5 & 0.5 \\
\hline orf19.3618 & $Y W P 1$ & cell-cell adhesion & 0.4 & 0.3 & 0.7 & 0.7 & 0.1 & 0.0 \\
\hline orf19.6146 & $C L G 1$ & cell cycle & 0.4 & 0.5 & 0.6 & 1.0 & 1.5 & 1.0 \\
\hline orf19.2187 & $A L G 7$ & aerobic respiration & 0.4 & 0.4 & 0.4 & 0.7 & 0.5 & 0.4 \\
\hline orf19.4520 & & D-gluconate metabolic process & 0.4 & 0.2 & 0.6 & 0.6 & 0.5 & 0.6 \\
\hline orf19.4390 & & & 0.4 & 0.3 & 1.1 & 1.8 & 0.3 & 0.2 \\
\hline orf19.3146 & & & 0.4 & 0.4 & 0.7 & 0.7 & 0.2 & 0.2 \\
\hline orf19.568 & SPE2 & pantothenate biosynthetic process & 0.4 & 0.2 & 1.4 & 2.0 & 2.1 & 1.4 \\
\hline orf19.6424 & & $\mathrm{G} 2 / \mathrm{M}$ transition of mitotic cell cycle & 0.4 & 0.2 & 0.3 & 0.5 & 0.3 & 0.9 \\
\hline orf19.5789 & $A D E 8$ & adenine biosynthetic process & 0.4 & 0.2 & 1.3 & 1.9 & 0.6 & 0.6 \\
\hline orf19.6691 & & drug transmembrane transport & 0.4 & 0.4 & 0.6 & 1.3 & 1.8 & 1.4 \\
\hline orf19.7564 & $D P B 2$ & DNA repair & 0.4 & 0.3 & 1.1 & 2.6 & 0.6 & 0.3 \\
\hline orf19.5612 & BMT4 & beta-1,2-oligomannoside metabolic process & 0.4 & 0.3 & 0.7 & 0.6 & 1.0 & 0.6 \\
\hline orf19.6562 & RNH35 & DNA replication, removal of RNA primer & 0.4 & 0.3 & 0.2 & 0.4 & 0.2 & 0.3 \\
\hline orf19.3810 & & folic acid and derivative biosynthetic process & 0.4 & 0.3 & 1.1 & 1.9 & 1.2 & 0.7 \\
\hline orf19.4783 & & & 0.4 & 0.2 & 0.3 & 0.2 & 0.2 & 0.3 \\
\hline orf19.3099 & TRP4 & tryptophan biosynthetic process & 0.4 & 0.4 & 0.8 & 1.9 & 0.2 & 0.3 \\
\hline orf19.2529 & & GPI anchor biosynthetic process & 0.4 & 0.4 & 0.9 & 1.4 & 0.8 & 0.6 \\
\hline orf19.3430 & & & 0.4 & 0.4 & 0.7 & 0.9 & 0.3 & 0.3 \\
\hline orf19.4910 & FGR41 & filamentous growth & 0.4 & 0.4 & 0.1 & 0.2 & 0.0 & 0.0 \\
\hline orf19.3469 & & regulation of transcription involved in $\mathrm{G} 1 / \mathrm{S}$ phase & 0.4 & 0.4 & 0.6 & 0.8 & 0.9 & 0.6 \\
\hline orf19.4241 & & & 0.4 & 0.3 & 0.5 & 0.6 & 0.6 & 0.7 \\
\hline orf19.5369 & & heme biosynthetic process & 0.4 & 0.4 & 0.7 & 0.8 & 0.7 & 0.7 \\
\hline
\end{tabular}


Table A-2. (Continued)

\begin{tabular}{|c|c|c|c|c|c|c|c|c|}
\hline \multirow[b]{3}{*}{$\begin{array}{c}\text { orf19 } \\
\text { Designation }\end{array}$} & \multirow[b]{3}{*}{$\begin{array}{l}\text { CGD } \\
\text { Name }\end{array}$} & \multirow[b]{3}{*}{ Process $^{a}$} & \multicolumn{6}{|c|}{ Change in Fold Expression } \\
\hline & & & \multicolumn{2}{|c|}{$\begin{array}{c}\text { SC5314+FLC } \\
\text { /SC5314 } \\
\end{array}$} & \multicolumn{2}{|c|}{$\begin{array}{c}\operatorname{cas} 5 \Delta / \Delta+\mathrm{FLC} \\
/ \operatorname{cas} 5 \Delta / \Delta \\
\end{array}$} & \multicolumn{2}{|c|}{$\begin{array}{c}u p c 2 \Delta / \Delta+\mathrm{FLC} / \\
u p c 2 \Delta / \Delta\end{array}$} \\
\hline & & & Exp1 & Exp2 & Exp1 & Exp2 & Exp1 & Exp2 \\
\hline orf19.6008 & & & 0.4 & 0.4 & 0.4 & 0.5 & 0.1 & 0.1 \\
\hline orf19.1757 & & regulation of transcription, DNA-dependent & 0.4 & 0.2 & 1.6 & 3.3 & 0.7 & 0.6 \\
\hline orf19.3591 & APE3 & vacuolar protein catabolic process & 0.4 & 0.4 & 0.6 & 0.7 & 0.9 & 0.5 \\
\hline orf19.7637 & $Y H B 4$ & response to stress & 0.4 & 0.5 & 0.3 & 0.2 & 1.0 & 1.5 \\
\hline orf19.3139 & & oxidation-reduction process & 0.4 & 0.3 & 0.3 & 0.3 & 0.1 & 0.3 \\
\hline orf19.1167 & & sulfur compound catabolic process & 0.4 & 0.1 & 1.4 & 1.1 & 0.3 & 0.2 \\
\hline orf19.7106 & VPS70 & protein targeting to vacuole & 0.4 & 0.5 & 1.0 & 1.1 & 3.9 & 1.0 \\
\hline orf19.5455 & & microautophagy & 0.4 & 0.2 & 0.4 & 0.3 & 0.3 & 0.3 \\
\hline orf19.7667 & IAH1 & filamentous growth & 0.4 & 0.4 & 0.5 & 0.5 & 0.2 & 0.4 \\
\hline orf19.4195.1 & FCA1 & cytosine metabolic process & 0.4 & 0.3 & 0.2 & 0.3 & 0.2 & 0.4 \\
\hline orf19.4177 & HIS5 & histidine biosynthetic process & 0.4 & 0.5 & 1.1 & 1.2 & 0.9 & 0.9 \\
\hline orf19.7149 & & endocytosis & 0.4 & 0.4 & 0.9 & 0.9 & 0.4 & 0.3 \\
\hline orf19.6977 & $G P I 1$ & GPI anchor biosynthetic process & 0.4 & 0.5 & 0.4 & 0.3 & 0.2 & 0.4 \\
\hline orf19.4666 & & & 0.4 & 0.5 & 1.0 & 1.8 & 1.1 & 1.1 \\
\hline orf19.5280 & MUP1 & sulfur amino acid transport & 0.4 & 0.4 & 0.5 & 0.6 & 0.3 & 0.4 \\
\hline orf19.4499 & RIM2 & mitochondrial genome maintenance & 0.4 & 0.3 & 0.7 & 1.0 & 0.3 & 0.6 \\
\hline orf19.5495 & & & 0.4 & 0.5 & 1.3 & 1.4 & 1.3 & 0.7 \\
\hline orf19.1049 & & & 0.4 & 0.4 & 0.7 & 1.0 & 0.4 & 0.5 \\
\hline orf19.5057 & & & 0.4 & 0.3 & 5.8 & 1.9 & 1.8 & 0.7 \\
\hline orf19.1332 & SNG4 & & 0.4 & 0.3 & 0.4 & 0.4 & 0.7 & 0.6 \\
\hline orf19.3192 & STI1 & protein complex assembly & 0.4 & 0.5 & 0.6 & 0.8 & 0.5 & 0.5 \\
\hline orf19.2041 & & chromatin remodeling & 0.4 & 0.5 & 1.0 & 1.1 & 0.5 & 0.4 \\
\hline orf19.2936 & & & 0.4 & 0.3 & 1.2 & 1.6 & 1.9 & 0.7 \\
\hline orf19.1559 & HOM2 & methionine metabolic process & 0.4 & 0.3 & 0.5 & 0.4 & 0.3 & 0.5 \\
\hline orf19.5136 & & & 0.4 & 0.5 & 0.1 & 0.1 & 0.1 & 0.3 \\
\hline orf19.1971 & $T R Y 3$ & positive regulation of cell-substrate adhesion & 0.4 & 0.5 & 0.5 & 0.3 & 0.2 & 0.7 \\
\hline orf19.3104 & $Y D C 1$ & ceramide metabolic process & 0.4 & 0.3 & 0.7 & 0.6 & 1.0 & 0.5 \\
\hline
\end{tabular}


Table A-2. (Continued)

\begin{tabular}{|c|c|c|c|c|c|c|c|c|}
\hline \multirow[b]{3}{*}{$\begin{array}{c}\text { orf19 } \\
\text { Designation }\end{array}$} & \multirow[b]{3}{*}{$\begin{array}{c}\text { CGD } \\
\text { Name }\end{array}$} & \multirow[b]{3}{*}{ Process $^{\mathrm{a}}$} & \multicolumn{6}{|c|}{ Change in Fold Expression } \\
\hline & & & \multicolumn{2}{|c|}{$\begin{array}{c}\text { SC5314+FLC } \\
\text { /SC5314 }\end{array}$} & \multicolumn{2}{|c|}{$\begin{array}{c}\operatorname{cas} 5 \Delta / \Delta+\mathrm{FLC} \\
/ \operatorname{cas} 5 \Delta / \Delta \\
\end{array}$} & \multicolumn{2}{|c|}{$\begin{array}{c}u p c 2 \Delta / \Delta+\mathrm{FLC} / \\
u p c 2 \Delta / \Delta\end{array}$} \\
\hline & & & Exp1 & Exp2 & Exp1 & Exp2 & Exp1 & Exp2 \\
\hline orf19.6010 & CDC5 & signal transduction during filamentous growth & 0.4 & 0.3 & 1.0 & 1.3 & 0.2 & 0.2 \\
\hline orf19.7023 & & chromatin remodeling & 0.4 & 0.3 & 0.5 & 0.5 & 0.1 & 0.4 \\
\hline orf19.5071 & $N R P 1$ & & 0.4 & 0.5 & 0.6 & 1.7 & 1.0 & 0.8 \\
\hline orf19.4284 & $B U R 2$ & mitotic sister chromatid segregation & 0.4 & 0.3 & 1.7 & 4.8 & 0.4 & 2.5 \\
\hline orf19.34 & GIT1 & phospholipid transport & 0.4 & 0.1 & 0.7 & 0.1 & 0.2 & 0.0 \\
\hline orf19.3016 & & meiotic chromosome segregation & 0.4 & 0.3 & 0.8 & 0.9 & 0.9 & 1.0 \\
\hline orf19.7324 & THI13 & thiamin biosynthetic process & 0.4 & 0.5 & 1.0 & 0.8 & 0.8 & 0.8 \\
\hline orf19.5839 & PDR17 & sterol biosynthetic process & 0.4 & 0.3 & 0.9 & 1.7 & 0.9 & 0.6 \\
\hline orf19.3394 & & & 0.4 & 0.4 & 0.4 & 0.4 & 0.8 & 0.7 \\
\hline orf19.6489 & FMP45 & cellular cell wall organization & 0.4 & 0.3 & 3.1 & 2.1 & 32.8 & 9.5 \\
\hline orf19.5745 & ALSO & cell adhesion & 0.4 & 0.4 & 0.2 & 0.1 & 0.3 & 0.3 \\
\hline orf19.3688 & & cell wall mannoprotein biosynthetic process & 0.4 & 0.5 & 1.3 & 1.5 & 0.7 & 1.4 \\
\hline orf19.3252 & DAL81 & positive regulation of cell adhesion & 0.4 & 0.3 & 1.3 & 1.6 & 0.8 & 0.9 \\
\hline orf19.4026 & HIS1 & histidine biosynthetic process & 0.4 & 0.4 & 0.7 & 0.7 & 0.4 & 0.7 \\
\hline orf19.103 & KAR5 & karyogamy involved in conjugation with cellular fusion & 0.4 & 0.4 & 1.0 & 1.5 & 0.7 & 2.6 \\
\hline orf19.6948 & $C C C 1$ & cellular calcium ion homeostasis & 0.4 & 0.3 & 0.7 & 0.7 & 1.7 & 0.9 \\
\hline orf19.3446 & & protein import into nucleus & 0.4 & 0.3 & 0.5 & 0.6 & 0.2 & 0.7 \\
\hline orf19.514 & SNP3 & nuclear mRNA splicing, via spliceosome & 0.4 & 0.2 & 0.4 & 0.5 & 0.1 & 0.3 \\
\hline orf19.7479 & NTH1 & trehalose catabolic process & 0.4 & 0.5 & 0.3 & 0.3 & 0.3 & 0.5 \\
\hline orf19.687 & & & 0.4 & 0.2 & 0.8 & 1.3 & 0.7 & 0.5 \\
\hline orf19.908 & FEN12 & fatty acid elongation & 0.4 & 0.3 & 0.5 & 0.8 & 0.3 & 0.3 \\
\hline orf19.3211 & $R C F 3$ & leading strand elongation & 0.4 & 0.4 & 0.8 & 1.2 & 0.3 & 0.4 \\
\hline orf19.708 & & microtubule nucleation & 0.4 & 0.4 & 0.7 & 0.9 & 0.5 & 0.4 \\
\hline orf19.348 & SKN2 & 1,6-beta-glucan biosynthetic process & 0.4 & 0.3 & 1.4 & 1.3 & 3.2 & 2.3 \\
\hline orf19.756 & $S A P 7$ & pathogenesis & 0.4 & 0.4 & 0.9 & 0.6 & 13.6 & 6.6 \\
\hline orf19.3322 & DUT1 & DNA replication & 0.4 & 0.4 & 0.8 & 0.8 & 0.3 & 0.2 \\
\hline orf19.1168 & $Z C F 3$ & filamentous growth & 0.4 & 0.1 & 1.5 & 2.2 & 0.5 & 1.0 \\
\hline
\end{tabular}


Table A-2. (Continued)

\begin{tabular}{|c|c|c|c|c|c|c|c|c|}
\hline \multirow{3}{*}{$\begin{array}{c}\text { orf19 } \\
\text { Designation }\end{array}$} & \multirow{3}{*}{$\begin{array}{c}\text { CGD } \\
\text { Name }\end{array}$} & \multirow[b]{3}{*}{ Process $^{\mathrm{a}}$} & \multicolumn{6}{|c|}{ Change in Fold Expression } \\
\hline & & & \multicolumn{2}{|c|}{$\begin{array}{c}\text { SC5314+FLC } \\
\text { /SC5314 } \\
\end{array}$} & \multicolumn{2}{|c|}{$\begin{array}{c}\operatorname{cas} 5 \Delta / \Delta+\mathrm{FLC} \\
/ \operatorname{cas} 5 \Delta / \Delta\end{array}$} & \multicolumn{2}{|c|}{$\begin{array}{c}\text { upc } 2 \Delta / \Delta+\mathrm{FLC} / \\
u p c 2 \Delta / \Delta\end{array}$} \\
\hline & & & Exp1 & Exp2 & Exp1 & Exp2 & Exp1 & Exp2 \\
\hline orf19.4447 & $Y M C 1$ & mitochondrial transport & 0.4 & 0.4 & 0.4 & 0.6 & 0.4 & 0.5 \\
\hline orf19.7279 & & nitrogen compound metabolic process & 0.4 & 0.2 & 1.0 & 1.3 & 0.4 & 0.3 \\
\hline orf19.927 & & telomere maintenance & 0.4 & 0.2 & 1.3 & 1.8 & 1.0 & 1.6 \\
\hline orf19.2686 & & nitrogen compound metabolic process & 0.4 & 0.5 & 1.1 & 1.1 & 0.7 & 0.4 \\
\hline orf19.6786 & & & 0.4 & 0.0 & 3.0 & 9.2 & 0.7 & 1.5 \\
\hline orf19.5242 & CDC6 & DNA replication initiation & 0.4 & 0.5 & 0.7 & 2.2 & 0.1 & 0.1 \\
\hline orf19.5736 & $A L S 5$ & cell adhesion & 0.4 & 0.3 & 0.7 & 1.1 & 3.0 & 2.2 \\
\hline orf19.7100 & & amino acid transport & 0.4 & 0.4 & 0.6 & 0.8 & 1.0 & 0.5 \\
\hline orf19.1832 & FCY23 & vitamin transport & 0.4 & 0.4 & 1.1 & 2.7 & 0.7 & 0.4 \\
\hline orf19.4301 & & chromatin silencing at rDNA & 0.4 & 0.4 & 1.0 & 2.8 & 1.1 & 0.2 \\
\hline orf19.7293 & MPS1 & mitotic cell cycle spindle assembly checkpoint & 0.4 & 0.5 & 1.6 & 4.5 & 0.8 & 0.7 \\
\hline orf19.321 & & amino acid transport & 0.4 & 0.5 & 0.4 & 0.4 & 0.9 & 0.6 \\
\hline orf19.6416 & & cellular response to oxidative stress & 0.4 & 0.4 & 2.2 & 3.3 & 3.8 & 2.0 \\
\hline orf19.4280 & & & 0.4 & 0.5 & 0.3 & 0.4 & 0.2 & 0.5 \\
\hline orf19.333 & $F C Y 2$ & drug transport & 0.4 & 0.4 & 0.2 & 0.3 & 0.1 & 0.2 \\
\hline orf19.398 & & & 0.4 & 0.4 & 1.3 & 1.2 & 1.2 & 1.3 \\
\hline orf19.4998 & $R O B 1$ & cellular amino acid catabolic process & 0.4 & 0.4 & 0.3 & 0.4 & 0.1 & 0.3 \\
\hline orf19.7344 & & chromosome segregation & 0.4 & 0.4 & 1.8 & 3.5 & 0.6 & 1.2 \\
\hline orf19.7427 & & & 0.4 & 0.3 & 0.7 & 1.1 & 1.9 & 1.3 \\
\hline orf19.1308 & & drug transport & 0.4 & 0.3 & 1.1 & 1.6 & 1.0 & 0.5 \\
\hline orf19.660 & & & 0.4 & 0.4 & 2.0 & 3.5 & 1.8 & 2.1 \\
\hline orf19.1318 & & & 0.4 & 0.2 & 0.8 & 1.3 & 0.6 & 0.6 \\
\hline orf19.4161 & & DNA repair & 0.4 & 0.4 & 2.4 & 4.3 & 0.6 & 0.3 \\
\hline orf19.4665 & & & 0.4 & 0.4 & 0.6 & 0.7 & 0.7 & 0.6 \\
\hline orf19.7554 & & drug transport & 0.5 & 0.3 & 1.1 & 2.0 & 10.9 & 11.4 \\
\hline orf19.1240 & & & 0.5 & 0.5 & 0.6 & 0.5 & 0.7 & 0.5 \\
\hline orf19.6784 & $P G A 32$ & cellular cell wall organization & 0.5 & 0.4 & 0.9 & 1.4 & 0.4 & 0.2 \\
\hline
\end{tabular}


Table A-2. (Continued)

\begin{tabular}{|c|c|c|c|c|c|c|c|c|}
\hline \multirow[b]{3}{*}{$\begin{array}{c}\text { orf19 } \\
\text { Designation }\end{array}$} & \multirow[b]{3}{*}{$\begin{array}{l}\text { CGD } \\
\text { Name }\end{array}$} & \multirow[b]{3}{*}{ Process $^{\mathrm{a}}$} & \multicolumn{6}{|c|}{ Change in Fold Expression } \\
\hline & & & \multicolumn{2}{|c|}{$\begin{array}{c}\text { SC5314+FLC } \\
\text { /SC5314 } \\
\end{array}$} & \multicolumn{2}{|c|}{$\begin{array}{c}\operatorname{cas} 5 \Delta / \Delta+\mathrm{FLC} \\
/ \operatorname{cas} 5 \Delta / \Delta \\
\end{array}$} & \multicolumn{2}{|c|}{$\begin{array}{c}u p c 2 \Delta / \Delta+\mathrm{FLC} / \\
u p c 2 \Delta / \Delta\end{array}$} \\
\hline & & & Exp1 & Exp2 & Exp1 & Exp2 & Exp1 & Exp2 \\
\hline orf19.28 & & thiamin pyrophosphate transport & 0.5 & 0.3 & 0.7 & 0.8 & 0.5 & 1.0 \\
\hline orf19.2951 & HOM6 & methionine metabolic process & 0.5 & 0.4 & 0.6 & 0.6 & 0.5 & 0.6 \\
\hline orf19.1238 & TUB4 & microtubule nucleation & 0.5 & 0.4 & 1.1 & 1.3 & 0.7 & 0.8 \\
\hline orf19.7083 & $D C C 1$ & mitotic sister chromatid cohesion & 0.5 & 0.4 & 0.9 & 1.6 & 0.5 & 0.5 \\
\hline orf19.2336 & & & 0.5 & 0.4 & 0.6 & 0.8 & 0.6 & 0.9 \\
\hline orf19.5074 & & protein sumoylation & 0.5 & 0.4 & 1.0 & 1.4 & 0.7 & 0.5 \\
\hline orf19.1948 & & & 0.5 & 0.4 & 0.8 & 0.8 & 0.4 & 0.4 \\
\hline orf19.4046 & & & 0.5 & 0.5 & 0.5 & 0.4 & 0.7 & 1.0 \\
\hline orf19.3788 & SPC34 & mitotic spindle organization in nucleus & 0.5 & 0.3 & 1.2 & 1.2 & 0.8 & 1.3 \\
\hline orf19.1613 & $I L V 2$ & branched chain family amino acid biosynthetic process & 0.5 & 0.5 & 0.5 & 0.7 & 0.2 & 0.2 \\
\hline orf19.3042 & & & 0.5 & 0.4 & 1.0 & 3.3 & 1.1 & 3.5 \\
\hline orf19.4040 & $I L V 3$ & branched chain family amino acid biosynthetic process & 0.5 & 0.5 & 0.4 & 0.3 & 0.1 & 0.2 \\
\hline orf19.1847 & ARO10 & aromatic amino acid family catabolic process to alcohol & 0.5 & 0.4 & 0.8 & 0.6 & 0.5 & 0.1 \\
\hline orf19.5917.3 & & mRNA export from nucleus & 0.5 & 0.4 & 0.4 & 0.4 & 0.2 & 0.2 \\
\hline orf19.2849 & $A Q Y 1$ & response to osmotic stress & 0.5 & 0.5 & 0.6 & 0.5 & 0.0 & 0.0 \\
\hline orf19.6177 & & & 0.5 & 0.3 & 0.8 & 0.9 & 0.2 & 0.4 \\
\hline orf19.6858 & $E D C 3$ & cytoplasmic mRNA processing body assembly & 0.5 & 0.4 & 1.8 & 2.0 & 0.6 & 1.1 \\
\hline orf19.547 & & DNA replication, removal of RNA primer & 0.5 & 0.3 & 1.8 & 8.1 & 1.3 & 0.5 \\
\hline orf19.1796 & & metabolic process & 0.5 & 0.4 & 0.3 & 0.3 & 0.3 & 0.5 \\
\hline orf19.6671 & $L A P 4$ & vacuolar protein catabolic process & 0.5 & 0.3 & 0.9 & 0.7 & 0.8 & 0.6 \\
\hline orf19.6189 & & cellular modified amino acid biosynthetic process & 0.5 & 0.5 & 0.5 & 0.6 & 1.0 & 1.1 \\
\hline orf19.4233 & THR4 & threonine metabolic process & 0.5 & 0.5 & 0.5 & 0.4 & 0.2 & 0.4 \\
\hline orf19.2547 & & & 0.5 & 0.2 & 1.7 & 2.7 & 0.9 & 1.0 \\
\hline orf19.704 & SOL3 & tRNA processing & 0.5 & 0.3 & 0.7 & 0.5 & 0.4 & 0.4 \\
\hline orf19.6936 & RAD53 & hyphal growth & 0.5 & 0.3 & 0.9 & 1.3 & 0.7 & 0.6 \\
\hline orf19.1626 & & peptidyl-lysine modification to hypusine & 0.5 & 0.4 & 0.3 & 0.4 & 0.1 & 0.3 \\
\hline orf19.6751 & & tRNA methylation & 0.5 & 0.3 & 0.7 & 1.6 & 0.5 & 1.3 \\
\hline
\end{tabular}


Table A-2. (Continued)

\begin{tabular}{|c|c|c|c|c|c|c|c|c|}
\hline \multirow[b]{3}{*}{$\begin{array}{c}\text { orf19 } \\
\text { Designation }\end{array}$} & \multirow[b]{3}{*}{$\begin{array}{l}\text { CGD } \\
\text { Name }\end{array}$} & \multirow[b]{3}{*}{ Process $^{\mathrm{a}}$} & \multicolumn{6}{|c|}{ Change in Fold Expression } \\
\hline & & & \multicolumn{2}{|c|}{$\begin{array}{c}\text { SC5314+FLC } \\
\text { /SC5314 } \\
\end{array}$} & \multicolumn{2}{|c|}{$\begin{array}{c}\operatorname{cas} 5 \Delta / \Delta+\mathrm{FLC} \\
/ \operatorname{cas} 5 \Delta / \Delta \\
\end{array}$} & \multicolumn{2}{|c|}{$\begin{array}{c}u p c 2 \Delta / \Delta+\mathrm{FLC} \\
u p c 2 \Delta / \Delta\end{array}$} \\
\hline & & & Exp1 & Exp2 & Exp1 & Exp2 & Exp1 & Exp2 \\
\hline orf19.7244 & & regulation of transcription, DNA-dependent & 0.5 & 0.5 & 0.8 & 0.9 & 0.3 & 0.6 \\
\hline orf19.6291 & & chromosome organization & 0.5 & 0.4 & 2.0 & 2.5 & 1.0 & 0.7 \\
\hline orf19.802 & $U G A 1$ & gamma-aminobutyric acid catabolic process & 0.5 & 0.5 & 1.5 & 2.0 & 0.5 & 0.6 \\
\hline orf19.105 & $H A L 22$ & methionine biosynthetic process & 0.5 & 0.5 & 0.9 & 0.7 & 0.7 & 0.5 \\
\hline orf19.1802 & & translational termination & 0.5 & 0.5 & 0.2 & 0.2 & 0.1 & 0.2 \\
\hline orf19.7375 & & nuclear mRNA splicing, via spliceosome & 0.5 & 0.3 & 1.3 & 1.8 & 1.1 & 2.1 \\
\hline orf19.7358 & SAM50 & protein complex assembly & 0.5 & 0.1 & 0.7 & 1.2 & 0.5 & 0.4 \\
\hline orf19.5647 & SUB2 & RNA elongation from RNA polymerase II promoter & 0.5 & 0.5 & 0.4 & 0.4 & 0.3 & 0.4 \\
\hline orf19.2637 & & response to unfolded protein & 0.5 & 0.3 & 0.5 & 0.6 & 0.4 & 0.4 \\
\hline orf19.4809 & $E R G 12$ & ergosterol biosynthetic process & 0.5 & 0.4 & 0.5 & 0.9 & 0.5 & 0.5 \\
\hline orf19.6365 & PTP1 & protein amino acid dephosphorylation & 0.5 & 0.4 & 0.4 & 0.5 & 0.9 & 1.1 \\
\hline orf19.2969 & RAD16 & nucleotide-excision repair, DNA damage recognition & 0.5 & 0.5 & 0.4 & 0.4 & 0.3 & 0.7 \\
\hline orf19.5825 & $N C B 2$ & negative regulation of transcription from RNA polymerase II & 0.5 & 0.2 & 0.7 & 0.7 & 0.4 & 0.6 \\
\hline orf19.7152 & & cysteine biosynthetic process & 0.5 & 0.5 & 0.8 & 0.7 & 0.7 & 0.6 \\
\hline orf19.6692 & $M N N 7$ & protein amino acid glycosylation & 0.5 & 0.4 & 0.9 & 1.1 & 0.9 & 0.6 \\
\hline orf19.3972 & & ER to Golgi vesicle-mediated transport & 0.5 & 0.4 & 1.2 & 1.6 & 1.3 & 1.0 \\
\hline orf19.5528 & $M O B 1$ & mitosis & 0.5 & 0.5 & 0.3 & 0.2 & 0.5 & 0.6 \\
\hline orf19.3914 & & translational initiation & 0.5 & 0.3 & 0.9 & 1.0 & 0.3 & 0.4 \\
\hline orf19.3089 & & cardiolipin metabolic process & 0.5 & 0.3 & 1.2 & 1.9 & 0.9 & 1.0 \\
\hline orf19.5841 & & & 0.5 & 0.5 & 0.7 & 0.8 & 0.8 & 1.0 \\
\hline orf19.5919 & $M E A 1$ & actin cortical patch localization & 0.5 & 0.3 & 1.1 & 1.4 & 0.8 & 0.5 \\
\hline orf19.5665 & & dehydro-D-arabinono-1,4-lactone biosynthetic process & 0.5 & 0.5 & 0.8 & 1.0 & 1.0 & 0.7 \\
\hline orf19.6755 & $D L D 2$ & aerobic respiration & 0.5 & 0.5 & 0.6 & 0.4 & 0.4 & 0.5 \\
\hline orf19.1358 & GCN4 & filamentous growth & 0.5 & 0.5 & 0.6 & 0.6 & 0.2 & 0.3 \\
\hline orf19.5034 & $Y B P 1$ & response to oxidative stress & 0.5 & 0.5 & 0.7 & 0.9 & 0.8 & 0.9 \\
\hline orf19.3870 & $A D E 13$ & purine base metabolic process & 0.5 & 0.4 & 1.0 & 0.9 & 0.2 & 0.2 \\
\hline orf19.4148 & & & 0.5 & 0.3 & 0.8 & 0.7 & 0.2 & 0.3 \\
\hline
\end{tabular}


Table A-2. (Continued)

\begin{tabular}{|c|c|c|c|c|c|c|c|c|}
\hline \multirow[b]{3}{*}{$\begin{array}{c}\text { orf19 } \\
\text { Designation }\end{array}$} & \multirow[b]{3}{*}{$\begin{array}{l}\text { CGD } \\
\text { Name }\end{array}$} & \multirow[b]{3}{*}{ Process $^{a}$} & \multicolumn{6}{|c|}{ Change in Fold Expression } \\
\hline & & & \multicolumn{2}{|c|}{$\begin{array}{c}\text { SC5314+FLC } \\
\text { /SC5314 } \\
\end{array}$} & \multicolumn{2}{|c|}{$\begin{array}{c}\operatorname{cas} 5 \Delta / \Delta+\mathrm{FLCC} \\
/ \operatorname{cas} 5 \Delta / \Delta\end{array}$} & \multicolumn{2}{|c|}{$\begin{array}{c}\text { upc } 2 \Delta / \Delta+\mathrm{FLC} / \\
\text { upc } 2 \Delta / \Delta\end{array}$} \\
\hline & & & Exp1 & $\operatorname{Exp2}$ & Exp1 & Exp2 & $\operatorname{Exp} 1$ & Exp2 \\
\hline orf19.4612 & & & 0.5 & 0.2 & 0.2 & 0.3 & 0.8 & 3.7 \\
\hline orf19.3141 & & ER to Golgi vesicle-mediated transport & 0.5 & 0.5 & 1.1 & 1.7 & 0.7 & 0.6 \\
\hline orf19.2763 & & meiotic telomere clustering & 0.5 & 0.4 & 1.7 & 1.7 & 0.8 & 0.9 \\
\hline orf19.1115 & GUK1 & GMP metabolic process & 0.5 & 0.5 & 0.7 & 1.1 & 0.2 & 0.2 \\
\hline orf19.6399 & ATS1 & budding cell bud growth & 0.5 & 0.4 & 0.9 & 0.9 & 1.5 & 1.5 \\
\hline orf19.4774 & $A O X 1$ & aerobic respiration & 0.5 & 0.4 & 1.3 & 1.2 & 1.6 & 1.1 \\
\hline orf19.6237 & $R A C 1$ & filamentous growth & 0.5 & 0.3 & 0.8 & 1.1 & 0.5 & 0.6 \\
\hline orf19.2092 & & methionine biosynthetic process & 0.5 & 0.5 & 0.6 & 0.8 & 0.8 & 0.7 \\
\hline orf19.6737 & & & 0.5 & 0.4 & 1.0 & 1.2 & 0.9 & 0.6 \\
\hline orf19.5572 & & protein amino acid N-linked glycosylation & 0.5 & 0.5 & 0.4 & 0.4 & 1.1 & 0.8 \\
\hline orf19.5648 & & & 0.5 & 0.3 & 0.8 & 1.2 & 0.5 & 0.7 \\
\hline orf19.3471 & & regulation of mitotic metaphase/anaphase transition & 0.5 & 0.4 & 0.7 & 0.5 & 0.3 & 0.7 \\
\hline orf19.1986 & ARO2 & aromatic amino acid family biosynthetic process & 0.5 & 0.5 & 0.5 & 0.6 & 0.2 & 0.3 \\
\hline orf19.939 & NAM7 & intracellular mRNA localization & 0.5 & 0.5 & 1.2 & 1.4 & 0.9 & 0.7 \\
\hline orf19.6628 & & chromosome segregation & 0.5 & 0.4 & 1.2 & 1.1 & 0.3 & 0.9 \\
\hline orf19.3364 & & & 0.5 & 0.4 & 0.7 & 0.7 & 0.4 & 0.5 \\
\hline orf19.3965 & & double-strand break repair via homologous recombination & 0.5 & 0.5 & 0.5 & 0.8 & 0.7 & 0.6 \\
\hline orf19.3449.2 & & cardiolipin biosynthetic process & 0.5 & 0.3 & 0.7 & 0.7 & 0.3 & 0.4 \\
\hline orf19.2693 & GST2 & regulation of nitrogen utilization & 0.5 & 0.3 & 1.1 & 0.7 & 0.5 & 0.3 \\
\hline orf19.3964 & ASH2 & chromatin silencing at telomere & 0.5 & 0.4 & 1.1 & 1.9 & 0.8 & 0.7 \\
\hline orf19.2213 & & & 0.5 & 0.4 & 1.8 & 3.0 & 0.7 & 1.4 \\
\hline orf19.1235 & HOM3 & methionine metabolic process & 0.5 & 0.5 & 0.3 & 0.6 & 0.2 & 0.4 \\
\hline orf19.6952 & & gene silencing by RNA & 0.5 & 0.5 & 0.5 & 0.4 & 0.4 & 0.6 \\
\hline orf19.1852 & & & 0.5 & 0.4 & 0.6 & 1.0 & 0.3 & 0.4 \\
\hline orf19.6167 & $A Y R 1$ & phosphatidic acid biosynthetic process & 0.5 & 0.5 & 0.6 & 0.9 & 0.8 & 0.6 \\
\hline orf19.390 & $C D C 42$ & cell budding & 0.5 & 0.5 & 0.8 & 1.0 & 0.9 & 0.7 \\
\hline orf19.7499 & & nicotinate nucleotide salvage & 0.5 & 0.5 & 0.3 & 0.4 & 0.4 & 0.5 \\
\hline
\end{tabular}


Table A-2. (Continued)

\begin{tabular}{|c|c|c|c|c|c|c|c|c|}
\hline \multirow[b]{3}{*}{$\begin{array}{c}\text { orf19 } \\
\text { Designation }\end{array}$} & \multirow[b]{3}{*}{$\begin{array}{l}\text { CGD } \\
\text { Name }\end{array}$} & \multirow[b]{3}{*}{ Process $^{a}$} & \multicolumn{6}{|c|}{ Change in Fold Expression } \\
\hline & & & \multicolumn{2}{|c|}{$\begin{array}{c}\text { SC5314+FLC } \\
\text { /SC5314 } \\
\end{array}$} & \multicolumn{2}{|c|}{$\begin{array}{c}\operatorname{cas} 5 \Delta / \Delta+\mathrm{FLC} \\
/ \operatorname{cas} 5 \Delta / \Delta \\
\end{array}$} & \multicolumn{2}{|c|}{$\begin{array}{c}\text { upc } 2 \Delta / \Delta+\mathrm{FLC} / \\
u p c 2 \Delta / \Delta \\
\end{array}$} \\
\hline & & & Exp1 & Exp2 & Exp1 & Exp2 & Exp1 & Exp2 \\
\hline orf19.7545 & & & 0.5 & 0.5 & 0.6 & 1.0 & 0.8 & 1.0 \\
\hline orf19.1600 & & & 0.5 & 0.3 & 0.8 & 2.5 & 1.0 & 0.4 \\
\hline orf19.6929 & & & 0.5 & 0.3 & 0.4 & 0.4 & 0.2 & 0.3 \\
\hline orf19.7484 & $A D E 1$ & adenine biosynthetic process & 0.5 & 0.3 & 0.8 & 0.8 & 0.3 & 0.3 \\
\hline orf19.3894 & & response to drug & 0.5 & 0.2 & 2.5 & 5.1 & 2.9 & 3.8 \\
\hline orf19.7615 & & ER to Golgi vesicle-mediated transport & 0.5 & 0.2 & 1.4 & 3.3 & 1.4 & 1.2 \\
\hline orf19.4523 & & folic acid and derivative biosynthetic process & 0.5 & 0.3 & 0.4 & 0.3 & 0.4 & 1.0 \\
\hline orf19.7017 & $Y O X 1$ & regulation of mitotic cell cycle & 0.5 & 0.4 & 2.2 & 3.1 & 2.0 & 1.3 \\
\hline orf19.6400 & & inositol metabolic process & 0.5 & 0.5 & 1.2 & 1.5 & 1.3 & 1.2 \\
\hline orf19.2533.1 & & cotranslational protein targeting to membrane & 0.5 & 0.3 & 0.9 & 1.1 & 0.4 & 0.6 \\
\hline orf19.3459 & & ascospore formation & 0.5 & 0.5 & 0.8 & 0.7 & 1.0 & 0.7 \\
\hline orf19.7097 & & response to drug & 0.5 & 0.4 & 0.9 & 1.3 & 0.7 & 0.8 \\
\hline orf19.4880 & & & 0.5 & 0.5 & 1.3 & 1.2 & 1.0 & 0.5 \\
\hline orf19.6976 & & folic acid transport & 0.5 & 0.3 & 2.1 & 2.5 & 1.0 & 0.4 \\
\hline orf19.3395 & & drug transport & 0.5 & 0.4 & 0.3 & 0.2 & 0.6 & 0.4 \\
\hline orf19.2870 & & & 0.5 & 0.4 & 4.1 & 4.6 & 1.5 & 1.1 \\
\hline orf19.1959 & & protein deubiquitination & 0.5 & 0.3 & 1.3 & 2.3 & 0.3 & 1.1 \\
\hline orf19.4580 & & & 0.5 & 0.3 & 0.3 & 0.2 & 0.1 & 0.5 \\
\hline orf19.2546 & $T R P 2$ & tryptophan biosynthetic process & 0.5 & 0.5 & 0.6 & 0.6 & 0.2 & 0.4 \\
\hline orf19.6875 & VPS35 & protein retention in Golgi apparatus & 0.5 & 0.5 & 0.8 & 0.7 & 0.6 & 0.5 \\
\hline orf19.5211 & $I D P 1$ & glutamate biosynthetic process & 0.5 & 0.5 & 0.9 & 0.9 & 0.3 & 0.3 \\
\hline orf19.6017 & & & 0.5 & 0.4 & 1.4 & 1.2 & 0.4 & 0.6 \\
\hline orf19.6316 & & L-amino acid transport & 0.5 & 0.3 & 0.8 & 0.8 & 0.8 & 0.5 \\
\hline orf19.6885 & SPO7 & meiosis & 0.5 & 0.5 & 1.0 & 1.5 & 1.7 & 1.3 \\
\hline orf19.1782 & & & 0.5 & 0.3 & 0.5 & 0.6 & 0.2 & 0.3 \\
\hline orf19.3872 & & & 0.5 & 0.4 & 0.5 & 0.5 & 1.1 & 1.0 \\
\hline orf19.2267 & $R F A 2$ & DNA strand elongation during DNA replication & 0.5 & 0.5 & 0.9 & 0.8 & 0.4 & 0.3 \\
\hline
\end{tabular}


Table A-2. (Continued)

\begin{tabular}{|c|c|c|c|c|c|c|c|c|}
\hline \multirow{3}{*}{$\begin{array}{c}\text { orf19 } \\
\text { Designation }\end{array}$} & \multirow{3}{*}{$\begin{array}{l}\text { CGD } \\
\text { Name }\end{array}$} & \multirow[b]{3}{*}{ Process $^{\mathrm{a}}$} & \multicolumn{6}{|c|}{ Change in Fold Expression } \\
\hline & & & \multicolumn{2}{|c|}{$\begin{array}{c}\text { SC5314+FLC } \\
\text { /SC5314 } \\
\end{array}$} & \multicolumn{2}{|c|}{$\begin{array}{c}\operatorname{cas} 5 \Delta / \Delta+\mathrm{FLC} \\
/ \operatorname{cas} 5 \Delta / \Delta\end{array}$} & \multicolumn{2}{|c|}{$\begin{array}{c}u p c 2 \Delta / \Delta+\mathrm{FLC} / \\
u p c 2 \Delta / \Delta\end{array}$} \\
\hline & & & Exp1 & Exp2 & Exp1 & Exp2 & Exp1 & Exp2 \\
\hline orf19.7454 & TAF60 & chromatin organization & 0.5 & 0.4 & 1.1 & 1.3 & 0.7 & 0.7 \\
\hline orf19.4036 & APM1 & filamentous growth & 0.5 & 0.5 & 1.2 & 1.8 & 1.2 & 0.9 \\
\hline orf19.2581 & & cellular metabolic process & 0.5 & 0.5 & 0.7 & 1.3 & 0.4 & 0.6 \\
\hline orf19.4749 & & & 0.5 & 0.5 & 1.0 & 1.1 & 0.8 & 0.3 \\
\hline orf19.4616 & POL30 & base-excision repair & 0.5 & 0.5 & 0.6 & 0.6 & 0.2 & 0.2 \\
\hline orf19.3442 & & oxidation-reduction process & 0.5 & 0.5 & 0.5 & 0.4 & 0.5 & 0.8 \\
\hline orf19.985 & & & 0.5 & 0.4 & 1.4 & 1.9 & 0.6 & 0.7 \\
\hline orf19.2040 & & hydrogen peroxide-mediated programmed cell death & 0.5 & 0.3 & 1.2 & 1.4 & 1.1 & 1.4 \\
\hline orf19.7638 & PRO1 & proline biosynthetic process & 0.5 & 0.5 & 0.3 & 0.3 & 0.5 & 1.0 \\
\hline orf19.4205.1 & & nuclear mRNA splicing, via spliceosome & 0.5 & 0.4 & 0.4 & 0.6 & 0.4 & 0.6 \\
\hline orf19.2461 & PRN4 & & 0.5 & 0.3 & 1.4 & 1.2 & 1.6 & 2.0 \\
\hline orf19.2949 & & intracellular signal transduction & 0.5 & 0.5 & 1.6 & 2.3 & 1.0 & 0.6 \\
\hline orf19.6650 & & & 0.5 & 0.4 & 1.1 & 1.5 & 1.8 & 2.1 \\
\hline orf19.7095 & & & 0.5 & 0.5 & 0.3 & 0.4 & 0.5 & 0.5 \\
\hline orf19.6635 & & & 0.5 & 0.4 & 1.1 & 1.0 & 0.9 & 1.7 \\
\hline orf19.1913 & & & 0.5 & 0.4 & 0.5 & 0.7 & 0.4 & 0.7 \\
\hline orf19.5628 & & dicarboxylic acid transport & 0.5 & 0.3 & 0.2 & 0.2 & 0.3 & 0.5 \\
\hline orf19.4022 & SDH4 & succinate metabolic process & 0.5 & 0.5 & 0.5 & 1.0 & 0.3 & 0.6 \\
\hline orf19.3761 & CDC54 & DNA replication initiation & 0.5 & 0.5 & 2.1 & 3.6 & 0.3 & 0.1 \\
\hline orf19.3679 & & nicotinamide nucleotide metabolic process & 0.5 & 0.4 & 0.6 & 0.5 & 0.6 & 0.6 \\
\hline orf19.4553 & & & 0.5 & 0.5 & 1.0 & 1.3 & 1.2 & 0.7 \\
\hline orf19.511 & & nicotinamide riboside metabolic process & 0.5 & 0.4 & 0.6 & 0.8 & 0.4 & 0.6 \\
\hline orf19.7175 & HLJ1 & ER-associated protein catabolic process & 0.5 & 0.5 & 0.7 & 1.0 & 0.8 & 0.8 \\
\hline orf19.95 & & & 0.5 & 0.5 & 1.6 & 3.2 & 0.9 & 0.4 \\
\hline orf19.3040 & EHT1 & medium-chain fatty acid biosynthetic process & 0.5 & 0.5 & 0.3 & 0.2 & 0.2 & 0.3 \\
\hline orf19.5505 & HIS7 & histidine biosynthetic process & 0.5 & 0.4 & 1.0 & 1.1 & 0.4 & 0.5 \\
\hline orf19.4748 & & nuclear mRNA splicing, via spliceosome & 0.5 & 0.4 & 0.8 & 0.9 & 0.4 & 0.9 \\
\hline
\end{tabular}


Table A-2. (Continued)

\begin{tabular}{|c|c|c|c|c|c|c|c|c|}
\hline \multirow{3}{*}{$\begin{array}{c}\text { orf19 } \\
\text { Designation }\end{array}$} & \multirow{3}{*}{$\begin{array}{c}\text { CGD } \\
\text { Name }\end{array}$} & \multirow[b]{3}{*}{ Process $^{\mathrm{a}}$} & \multicolumn{6}{|c|}{ Change in Fold Expression } \\
\hline & & & \multicolumn{2}{|c|}{$\begin{array}{c}\text { SC5314+FLC } \\
\text { /SC5314 }\end{array}$} & \multicolumn{2}{|c|}{$\begin{array}{c}\operatorname{cas} 5 \Delta / \Delta+\mathrm{FLC} \\
/ \operatorname{cas} 5 \Delta / \Delta\end{array}$} & \multicolumn{2}{|c|}{$\begin{array}{c}u p c 2 \Delta / \Delta+\mathrm{FLC} / \\
u p c 2 \Delta / \Delta\end{array}$} \\
\hline & & & Exp1 & Exp2 & Exp1 & Exp2 & Exp1 & Exp2 \\
\hline orf19.434 & PRD1 & proteolysis & 0.5 & 0.5 & 0.6 & 0.4 & 0.4 & 0.4 \\
\hline orf19.7631 & SLD5 & DNA-dependent DNA replication & 0.5 & 0.4 & 1.6 & 1.4 & 1.0 & 0.5 \\
\hline orf19.6779 & PRO2 & proline biosynthetic process & 0.5 & 0.4 & 1.5 & 1.8 & 0.9 & 0.8 \\
\hline orf19.1973 & HAP5 & filamentous growth & 0.5 & 0.3 & 1.2 & 1.4 & 0.4 & 0.6 \\
\hline orf19.1681 & & & 0.5 & 0.5 & 0.9 & 0.8 & 3.0 & 3.6 \\
\hline orf19.3844 & $M R P 8$ & translation & 0.5 & 0.5 & 0.6 & 0.5 & 0.7 & 1.3 \\
\hline orf19.306 & USO5 & ER to Golgi vesicle-mediated transport & 0.5 & 0.5 & 1.8 & 2.0 & 1.3 & 1.7 \\
\hline orf19.4270 & & protein amino acid O-linked glycosylation & 0.5 & 0.5 & 1.4 & 1.2 & 1.1 & 0.9 \\
\hline orf19.993 & & & 0.5 & 0.2 & 0.9 & 0.9 & 0.4 & 0.3 \\
\hline orf19.2898 & & protein targeting to vacuole & 0.5 & 0.4 & 1.5 & 1.8 & 6.3 & 3.1 \\
\hline orf19.3534 & RHO3 & hyphal growth & 0.5 & 0.4 & 0.9 & 1.2 & 1.3 & 0.9 \\
\hline orf19.4817 & $R A M 2$ & protein geranylgeranylation & 0.5 & 0.3 & 0.5 & 0.5 & 0.3 & 0.6 \\
\hline orf19.244 & $D C G 1$ & nitrogen compound metabolic process & 0.5 & 0.3 & 1.0 & 0.9 & 0.6 & 0.4 \\
\hline orf19.7370 & & & 0.5 & 0.4 & 1.5 & 2.4 & 2.5 & 1.6 \\
\hline orf19.4575 & & & 0.5 & 0.3 & 0.5 & 0.8 & 0.2 & 0.2 \\
\hline orf19.7304 & & & 0.5 & 0.5 & 0.9 & 0.9 & 0.8 & 0.4 \\
\hline orf19.3715 & $A S F 1$ & DNA replication-dependent nucleosome assembly & 0.5 & 0.5 & 1.0 & 2.1 & 0.3 & 0.2 \\
\hline orf19.424 & TRP99 & response to oxidative stress & 0.5 & 0.4 & 1.0 & 1.1 & 0.6 & 0.7 \\
\hline orf19.2923 & & drug transport & 0.5 & 0.5 & 0.5 & 0.4 & 0.9 & 0.7 \\
\hline orf19.6813 & & & 0.5 & 0.3 & 1.0 & 1.0 & 0.4 & 0.2 \\
\hline orf19.2087 & SAS2 & chromatin silencing at telomere & 0.5 & 0.4 & 1.3 & 2.2 & 0.7 & 0.7 \\
\hline orf19.6563 & & & 0.5 & 0.5 & 1.0 & 1.3 & 1.7 & 1.1 \\
\hline orf19.3043 & & lipid metabolic process & 0.5 & 0.4 & 0.9 & 1.0 & 0.9 & 0.8 \\
\hline orf19.4796 & & negative regulation of translation & 0.5 & 0.4 & 1.0 & 1.5 & 0.3 & 0.5 \\
\hline orf19.1999 & & & 0.5 & 0.4 & 0.5 & 0.3 & 0.6 & 1.1 \\
\hline orf19.1828 & BUD16 & cellular bud site selection & 0.5 & 0.3 & 1.3 & 3.1 & 1.0 & 0.9 \\
\hline orf19.5487 & $C D C 46$ & DNA replication initiation & 0.5 & 0.4 & 0.9 & 2.3 & 0.2 & 0.1 \\
\hline
\end{tabular}


Table A-2. (Continued)

\begin{tabular}{|c|c|c|c|c|c|c|c|c|}
\hline \multirow{3}{*}{$\begin{array}{c}\text { orf19 } \\
\text { Designation }\end{array}$} & \multirow{3}{*}{$\begin{array}{c}\text { CGD } \\
\text { Name }\end{array}$} & \multirow[b]{3}{*}{ Process $^{\mathrm{a}}$} & \multicolumn{6}{|c|}{ Change in Fold Expression } \\
\hline & & & \multicolumn{2}{|c|}{$\begin{array}{c}\text { SC5314+FLC } \\
\text { /SC5314 }\end{array}$} & \multicolumn{2}{|c|}{$\begin{array}{c}\operatorname{cas} 5 \Delta / \Delta+\mathrm{FLC} \\
/ \operatorname{cas} 5 \Delta / \Delta\end{array}$} & \multicolumn{2}{|c|}{$\begin{array}{c}u p c 2 \Delta / \Delta+\mathrm{FLC} / \\
u p c 2 \Delta / \Delta\end{array}$} \\
\hline & & & Exp1 & Exp2 & Exp1 & Exp2 & Exp1 & Exp2 \\
\hline orf19.4024 & RIB5 & riboflavin biosynthetic process & 0.5 & 0.4 & 0.8 & 1.0 & 0.5 & 0.7 \\
\hline orf19.5363 & & dolichol-linked oligosaccharide biosynthetic process & 0.5 & 0.5 & 0.5 & 0.6 & 0.5 & 0.6 \\
\hline orf19.7414 & ALS6 & cell adhesion & 0.5 & 0.4 & 0.7 & 1.1 & 0.7 & 0.6 \\
\hline orf19.1795.1 & & protein targeting to ER & 0.5 & 0.5 & 0.2 & 0.3 & 0.3 & 0.5 \\
\hline
\end{tabular}

${ }^{a}$ Descriptions are from the Candida Genome Database (http://www.candidagenome.org).

Red indicates those identified to be fluconazole repressed and dependent on Cas5 or Upc2. 


\section{APPENDIX B. CAS5 SUPPLEMENTAL DATA}

Table B-1. TF disruptant clones displaying a reduction in FLC MIC in RPMI, rescreened in YPD

\begin{tabular}{|c|c|c|c|c|c|c|}
\hline \multirow[b]{2}{*}{ Clone ID } & \multirow[b]{2}{*}{ CGD } & \multirow{2}{*}{$\begin{array}{c}\text { orf19 } \\
\text { Designation }\end{array}$} & \multicolumn{2}{|c|}{$\operatorname{MIC}(\mu \mathrm{g} / \mathrm{ml})$} & \multicolumn{2}{|c|}{$\operatorname{MFC}(\mu \mathrm{g} / \mathrm{ml})$} \\
\hline & & & RPMI & YPD & RPMI & YPD \\
\hline WT & -- & -- & 0.25 & 0.5 & $>4$ & $>64$ \\
\hline TF3 & RPN4 & orf19.1069 & 0.0625 & 0.25 & 0.125 & 32 \\
\hline TF4 & -- & orf19.1253 & $\mathrm{SG}^{\mathrm{a}}$ & 0.5 & SG & $>64$ \\
\hline TF33 & $C A S 5$ & orf19.4670 & 0.125 & 0.25 & 4 & 2 \\
\hline TF45 & $G A L 4$ & orf19.5338 & 0.125 & 0.5 & $>4$ & $>64$ \\
\hline TF59 & $A C E 2$ & orf19.6124 & 0.03125 & 0.5 & 2 & $>64$ \\
\hline TF77 & $U P C 2$ & orf19.391 & 0.03125 & 0.125 & 1 & 1 \\
\hline TF82 & STP4 & orf19.909 & 0.125 & 0.5 & $>4$ & $>64$ \\
\hline TF95 & NDT80 & orf19.2119 & 0.03125 & 0.5 & $>4$ & $>64$ \\
\hline TF103 & HFL1 & orf19.3063 & $\mathrm{NG}^{\mathrm{a}}$ & NG & NG & NG \\
\hline TF104 & CZF1 & orf19.3127 & 0.0625 & 0.5 & 0.25 & 8 \\
\hline TF126 & RIM101 & orf19.7247 & 0.0625 & 0.5 & $>4$ & 4 \\
\hline TF127 & $I S W 2$ & orf19.7401 & 0.0625 & 0.25 & $>4$ & 16 \\
\hline TF155 & $D A L 81$ & orf19.3252 & 0.0625 & 0.5 & 4 & $>64$ \\
\hline TF158 & $R A P 1$ & orf19.1773 & 0.03125 & 0.5 & $>4$ & $>64$ \\
\hline TF159 & -- & orf19.1757 & 0.03125 & 0.5 & $>4$ & $>64$ \\
\hline TF160 & -- & orf19.2743 & 0.03125 & 0.5 & $>4$ & $>64$ \\
\hline TF163 & $R F X 1$ & orf19.3865 & 0.0078125 & 0.25 & $>4$ & $>64$ \\
\hline TF164 & $R G T 1$ & orf19.2747 & 0.0625 & 0.5 & $>4$ & $>64$ \\
\hline TF165 & PPRI & orf19.3986 & 0.03125 & 0.25 & $>4$ & $>64$ \\
\hline
\end{tabular}

${ }^{a}$ Abbreviations: Slow growth (SG), No growth (NG) 
Table B-2. Genes downregulated by at least 0.5-fold by fluconazole which are dependent upon Cas5 and Upc2

\begin{tabular}{|c|c|c|c|c|c|c|c|c|c|c|c|c|}
\hline \multirow[b]{3}{*}{ Process $^{a}$} & \multirow[b]{3}{*}{$\begin{array}{c}\text { orf19 } \\
\text { Designation } \\
\end{array}$} & \multirow[b]{3}{*}{$\begin{array}{l}\text { CGD } \\
\text { Name } \\
\end{array}$} & \multicolumn{6}{|c|}{ Change in Fold Expression } & & & & \\
\hline & & & \multicolumn{2}{|c|}{$\begin{array}{c}\text { SC5314+FLC/ } \\
\text { SC5314 (A) }\end{array}$} & \multicolumn{2}{|c|}{$\begin{array}{c}\operatorname{cas} 5 \Delta / \Delta+\mathrm{FLC} / \\
\operatorname{cas} 5 \Delta / \Delta(\mathrm{B}) \\
\end{array}$} & \multicolumn{2}{|c|}{$\begin{array}{c}u p c 2 \Delta / \Delta+\mathrm{FLC} / \\
u p c 2 \Delta / \Delta(\mathrm{C})\end{array}$} & \multicolumn{2}{|c|}{$\begin{array}{c}C A S 5 \text { Ratio } \\
(\mathrm{B} / \mathrm{A}) \\
\end{array}$} & \multicolumn{2}{|c|}{$\begin{array}{c}\text { UPC2 Ratio } \\
\text { (C/A) }\end{array}$} \\
\hline & & & Exp1 & Exp2 & Exp1 & Exp2 & Exp1 & Exp2 & Exp1 & Exp2 & Exp1 & Exp2 \\
\hline \multirow{4}{*}{$\begin{array}{l}\text { Lipid metabolic } \\
\text { process }\end{array}$} & orf19.1887 & & 0.1 & 0.1 & 0.4 & 0.5 & 0.6 & 0.5 & 2.8 & 3.2 & 4.4 & 3.3 \\
\hline & orf19.3043 & & 0.5 & 0.4 & 0.9 & 1.0 & 0.9 & 0.8 & 1.6 & 2.6 & 1.6 & 2.0 \\
\hline & orf19.3089 & & 0.5 & 0.3 & 1.2 & 1.9 & 0.9 & 1.0 & 2.5 & 5.7 & 1.9 & 2.9 \\
\hline & orf19.3104 & $Y D C 1$ & 0.4 & 0.3 & 0.7 & 0.6 & 1.0 & 0.5 & 1.8 & 2.0 & 2.3 & 1.6 \\
\hline \multirow{2}{*}{$\begin{array}{c}\text { Cell wall } \\
\text { organization }\end{array}$} & orf19.5032 & SIM1 & 0.3 & 0.4 & 0.6 & 1.0 & 0.8 & 1.0 & 1.8 & 2.7 & 2.5 & 2.6 \\
\hline & orf19.6489 & $F M P 45$ & 0.4 & 0.3 & 3.1 & 2.1 & 32.8 & 9.5 & 7.3 & 6.9 & 78.0 & 31.0 \\
\hline Iron ion transport & orf19.6948 & $C C C 1$ & 0.4 & 0.3 & 0.7 & 0.7 & 1.7 & 0.9 & 1.7 & 2.1 & 4.0 & 2.7 \\
\hline \multirow[t]{4}{*}{ Drug transport } & orf19.1308 & & 0.4 & 0.3 & 1.1 & 1.6 & 1.0 & 0.5 & 2.4 & 5.8 & 2.3 & 1.6 \\
\hline & orf19.4889 & & 0.3 & 0.2 & 0.7 & 1.0 & 0.6 & 0.5 & 2.6 & 4.3 & 2.3 & 2.2 \\
\hline & orf19.6691 & & 0.4 & 0.4 & 0.6 & 1.3 & 1.8 & 1.4 & 1.6 & 3.7 & 4.7 & 4.0 \\
\hline & orf19.7554 & & 0.5 & 0.3 & 1.1 & 2.0 & 10.9 & 11.4 & 2.5 & 6.1 & 24.1 & 34.1 \\
\hline \multirow[t]{11}{*}{ Transport } & orf19.1182 & & 0.3 & 0.3 & 0.7 & 1.0 & 0.8 & 0.5 & 2.1 & 3.6 & 2.5 & 1.9 \\
\hline & orf19.2337 & $A L P 1$ & 0.1 & 0.1 & 0.3 & 0.4 & 0.4 & 0.3 & 3.2 & 7.5 & 5.0 & 6.1 \\
\hline & orf19.2738 & SUL2 & 0.1 & 0.3 & 1.7 & 1.2 & 5.4 & 0.8 & 12.8 & 4.7 & 40.5 & 3.1 \\
\hline & orf19.2898 & & 0.5 & 0.4 & 1.5 & 1.8 & 6.3 & 3.1 & 2.8 & 4.8 & 11.7 & 8.1 \\
\hline & orf19.3972 & & 0.5 & 0.4 & 1.2 & 1.6 & 1.3 & 1.0 & 2.5 & 3.7 & 2.7 & 2.4 \\
\hline & orf19.4456 & GAP4 & 0.2 & 0.3 & 0.5 & 0.7 & 0.9 & 0.6 & 1.9 & 2.2 & 3.6 & 1.8 \\
\hline & orf19.4733 & $Y M C 2$ & 0.3 & 0.3 & 0.9 & 0.9 & 0.9 & 0.4 & 2.9 & 3.3 & 2.7 & 1.7 \\
\hline & orf19.5017 & DUR32 & 0.1 & 0.1 & 0.7 & 0.6 & 0.9 & 0.2 & 6.8 & 4.6 & 8.7 & 1.7 \\
\hline & orf19.5663 & RCH1 & 0.3 & 0.2 & 0.9 & 0.8 & 1.3 & 1.6 & 3.1 & 3.9 & 4.5 & 8.2 \\
\hline & orf19.6316 & & 0.5 & 0.3 & 0.8 & 0.8 & 0.8 & 0.5 & 1.5 & 3.3 & 1.6 & 1.9 \\
\hline & orf19.6563 & & 0.5 & 0.5 & 1.0 & 1.3 & 1.7 & 1.1 & 1.9 & 2.4 & 3.0 & 2.1 \\
\hline
\end{tabular}


Table B-2. (Continued)

\begin{tabular}{|c|c|c|c|c|c|c|c|c|c|c|c|c|}
\hline \multirow[b]{3}{*}{ Process $^{\mathrm{a}}$} & \multirow{3}{*}{$\begin{array}{c}\text { orf19 } \\
\text { Designation } \\
\end{array}$} & \multirow{3}{*}{$\begin{array}{c}\text { CGD } \\
\text { Name }\end{array}$} & \multicolumn{6}{|c|}{ Change in Fold Expression } & \multirow{2}{*}{\multicolumn{2}{|c|}{$\begin{array}{c}\text { CAS5 Ratio } \\
\text { (B/A) }\end{array}$}} & \multirow{2}{*}{\multicolumn{2}{|c|}{$\begin{array}{c}\text { UPC2 Ratio } \\
(\mathrm{C} / \mathrm{A})\end{array}$}} \\
\hline & & & \multicolumn{2}{|c|}{$\begin{array}{c}\text { SC5314+FLC/ } \\
\text { SC5314 (A) }\end{array}$} & \multicolumn{2}{|c|}{$\begin{array}{c}\operatorname{cas} 5 \Delta / \Delta+\mathrm{FLC} / \\
\operatorname{cas} 5 \Delta / \Delta(\mathrm{B}) \\
\end{array}$} & \multicolumn{2}{|c|}{$\begin{array}{c}u p c 2 \Delta / \Delta+\mathrm{FLC} / \\
u p c 2 \Delta / \Delta(\mathrm{C})\end{array}$} & & & & \\
\hline & & & Exp1 & Exp2 & Exp1 & Exp2 & Exp1 & Exp2 & Exp1 & $\operatorname{Exp2}$ & Exp1 & $\operatorname{Exp2}$ \\
\hline & orf19.7106 & VPS70 & 0.4 & 0.5 & 1.0 & 1.1 & 3.9 & 1.0 & 2.4 & 2.4 & 9.7 & 2.2 \\
\hline & orf19.7370 & & 0.5 & 0.4 & 1.5 & 2.4 & 2.5 & 1.6 & 2.8 & 6.9 & 4.6 & 4.5 \\
\hline & orf19.7615 & & 0.5 & 0.2 & 1.4 & 3.3 & 1.4 & 1.2 & 2.8 & 13.2 & 2.7 & 4.9 \\
\hline \multirow[t]{4}{*}{ Response to stress } & orf19.4526 & HSP30 & 0.2 & 0.1 & 2.6 & 1.2 & 0.8 & 1.5 & 15.6 & 12.6 & 5.2 & 15.9 \\
\hline & orf19.5034 & $Y B P 1$ & 0.5 & 0.5 & 0.7 & 0.9 & 0.8 & 0.9 & 1.5 & 1.9 & 1.7 & 1.9 \\
\hline & orf19.6779 & PRO2 & 0.5 & 0.4 & 1.5 & 1.8 & 0.9 & 0.8 & 2.7 & 4.1 & 1.7 & 1.8 \\
\hline & orf19.946 & MET14 & 0.1 & 0.1 & 1.1 & 2.5 & 0.4 & 0.2 & 14.5 & 28.5 & 5.4 & 2.6 \\
\hline \multirow{6}{*}{$\begin{array}{l}\text { Response to } \\
\text { chemical stimulus }\end{array}$} & orf19.3106 & MET16 & 0.2 & 0.3 & 0.8 & 1.4 & 1.2 & 0.6 & 3.5 & 5.2 & 4.8 & 2.3 \\
\hline & orf19.3252 & $D A L 81$ & 0.4 & 0.3 & 1.3 & 1.6 & 0.8 & 0.9 & 3.1 & 4.6 & 1.8 & 2.6 \\
\hline & orf19.3894 & & 0.5 & 0.2 & 2.5 & 5.1 & 2.9 & 3.8 & 5.0 & 24.2 & 5.7 & 17.7 \\
\hline & orf19.4177 & HIS5 & 0.4 & 0.5 & 1.1 & 1.2 & 0.9 & 0.9 & 2.7 & 2.6 & 2.3 & 1.9 \\
\hline & orf 19.5839 & PDR17 & 0.4 & 0.3 & 0.9 & 1.7 & 0.9 & 0.6 & 2.2 & 5.3 & 2.1 & 2.0 \\
\hline & orf19.6671 & $L A P 4$ & 0.5 & 0.3 & 0.9 & 0.7 & 0.8 & 0.6 & 2.0 & 2.2 & 1.8 & 1.7 \\
\hline \multirow{4}{*}{$\begin{array}{l}\text { Oxidation- } \\
\text { reduction process }\end{array}$} & orf19.2040 & & 0.5 & 0.3 & 1.2 & 1.4 & 1.1 & 1.4 & 2.3 & 4.0 & 2.1 & 4.2 \\
\hline & orf19.3810 & & 0.4 & 0.3 & 1.1 & 1.9 & 1.2 & 0.7 & 2.9 & 5.6 & 3.0 & 2.1 \\
\hline & orf19.5665 & & 0.5 & 0.5 & 0.8 & 1.0 & 1.0 & 0.7 & 1.6 & 1.9 & 2.1 & 1.5 \\
\hline & orf19.7098 & & 0.2 & 0.1 & 0.9 & 0.8 & 0.6 & 0.9 & 4.4 & 5.7 & 2.7 & 7.0 \\
\hline \multirow{5}{*}{$\begin{array}{l}\text { Filamentous } \\
\text { growth }\end{array}$} & orf19.1253 & PHO4 & 0.3 & 0.2 & 1.0 & 1.5 & 0.8 & 0.6 & 3.1 & 6.5 & 2.5 & 2.8 \\
\hline & orf19.1621 & GPA2 & 0.4 & 0.5 & 0.7 & 0.8 & 0.9 & 1.0 & 2.0 & 1.6 & 2.6 & 1.8 \\
\hline & orf19.4036 & $A P M 1$ & 0.5 & 0.5 & 1.2 & 1.8 & 1.2 & 0.9 & 2.3 & 3.5 & 2.3 & 1.7 \\
\hline & orf19.5992 & WOR2 & 0.3 & 0.2 & 0.5 & 0.4 & 0.9 & 0.6 & 1.9 & 1.8 & 3.2 & 2.4 \\
\hline & orf19.7025 & $M C M 1$ & 0.3 & 0.4 & 0.5 & 0.6 & 0.5 & 0.7 & 1.9 & 1.7 & 1.8 & 1.8 \\
\hline
\end{tabular}




\section{Table B-2. (Continued)}

\begin{tabular}{|c|c|c|c|c|c|c|c|c|c|c|c|c|}
\hline \multirow[b]{3}{*}{ Process $^{\mathrm{a}}$} & \multirow[b]{3}{*}{$\begin{array}{c}\text { orf19 } \\
\text { Designation } \\
\end{array}$} & \multirow[b]{3}{*}{$\begin{array}{l}\text { CGD } \\
\text { Name } \\
\end{array}$} & \multicolumn{6}{|c|}{ Change in Fold Expression } & \multirow{2}{*}{\multicolumn{2}{|c|}{$\begin{array}{c}C A S 5 \text { Ratio } \\
\text { (B/A) }\end{array}$}} & \multirow{2}{*}{\multicolumn{2}{|c|}{$\begin{array}{c}\text { UPC2 Ratio } \\
\text { (C/A) }\end{array}$}} \\
\hline & & & \multicolumn{2}{|c|}{$\begin{array}{c}\text { SC5314+FLC/ } \\
\text { SC5314 (A) }\end{array}$} & \multicolumn{2}{|c|}{$\begin{array}{c}\operatorname{cas} 5 \Delta / \Delta+\mathrm{FLC} / \\
\operatorname{cas} 5 \Delta / \Delta(\mathrm{B})\end{array}$} & \multicolumn{2}{|c|}{$\begin{array}{c}\text { upc } 2 \Delta / \Delta+\mathrm{FLC} / \\
\operatorname{upc} 2 \Delta / \Delta(\mathrm{C})\end{array}$} & & & & \\
\hline & & & Exp1 & Exp2 & Exp1 & Exp2 & Exp1 & Exp2 & Exp1 & Exp2 & Exp1 & Exp2 \\
\hline & orf19.7324 & THI13 & 0.4 & 0.5 & 1.0 & 0.8 & 0.8 & 0.8 & 2.3 & 1.6 & 2.0 & 1.7 \\
\hline \multirow[t]{10}{*}{$\begin{array}{c}\text { Organelle } \\
\text { organization }\end{array}$} & orf19.103 & KAR5 & 0.4 & 0.4 & 1.0 & 1.5 & 0.7 & 2.6 & 2.4 & 4.2 & 1.5 & 7.2 \\
\hline & orf19.1238 & TUB4 & 0.5 & 0.4 & 1.1 & 1.3 & 0.7 & 0.8 & 2.4 & 3.0 & 1.6 & 1.9 \\
\hline & orf19.2600 & SPC98 & 0.4 & 0.4 & 1.6 & 3.7 & 1.7 & 1.3 & 4.5 & 9.8 & 4.8 & 3.5 \\
\hline & orf19.2763 & & 0.5 & 0.4 & 1.7 & 1.7 & 0.8 & 0.9 & 3.5 & 3.8 & 1.7 & 1.9 \\
\hline & orf19.3534 & RHO3 & 0.5 & 0.4 & 0.9 & 1.2 & 1.3 & 0.9 & 1.7 & 3.0 & 2.4 & 2.3 \\
\hline & orf19.3688 & & 0.4 & 0.5 & 1.3 & 1.5 & 0.7 & 1.4 & 3.0 & 3.3 & 1.6 & 3.0 \\
\hline & orf19.3788 & SPC34 & 0.5 & 0.3 & 1.2 & 1.2 & 0.8 & 1.3 & 2.6 & 3.6 & 1.8 & 3.7 \\
\hline & orf19.6291 & & 0.5 & 0.4 & 2.0 & 2.5 & 1.0 & 0.7 & 4.3 & 6.3 & 2.1 & 1.7 \\
\hline & orf19.6399 & ATS1 & 0.5 & 0.4 & 0.9 & 0.9 & 1.5 & 1.5 & 1.9 & 2.6 & 3.0 & 4.2 \\
\hline & orf19.6885 & SPO7 & 0.5 & 0.5 & 1.0 & 1.5 & 1.7 & 1.3 & 1.8 & 3.1 & 3.3 & 2.8 \\
\hline \multirow[t]{5}{*}{ Cell cycle } & orf19.1828 & BUD16 & 0.5 & 0.3 & 1.3 & 3.1 & 1.0 & 0.9 & 2.4 & 10.9 & 1.8 & 3.3 \\
\hline & orf19.3016 & & 0.4 & 0.3 & 0.8 & 0.9 & 0.9 & 1.0 & 1.9 & 3.0 & 2.1 & 3.6 \\
\hline & orf19.3230 & BOI2 & 0.4 & 0.4 & 1.0 & 1.4 & 1.5 & 0.9 & 2.7 & 3.2 & 4.1 & 2.1 \\
\hline & orf19.5919 & $M E A 1$ & 0.3 & 1.1 & 1.4 & 0.8 & 0.5 & 2.3 & 4.4 & 1.6 & 1.6 & \\
\hline & orf19.7017 & $Y O X 1$ & 0.5 & 0.4 & 2.2 & 3.1 & 2.0 & 1.3 & 4.4 & 8.2 & 4.0 & 3.5 \\
\hline \multirow{5}{*}{$\begin{array}{c}\text { DNA metabolic } \\
\text { process }\end{array}$} & orf19.3964 & ASH2 & 0.5 & 0.4 & 1.1 & 1.9 & 0.8 & 0.7 & 2.1 & 4.9 & 1.6 & 1.8 \\
\hline & orf19.547 & & 0.5 & 0.3 & 1.8 & 8.1 & 1.3 & 0.5 & 3.9 & 25.0 & 2.9 & 1.6 \\
\hline & orf19.7190 & $O G G 1$ & 0.4 & 0.4 & 3.5 & 5.1 & 1.6 & 0.6 & 9.4 & 13.9 & 4.3 & 1.6 \\
\hline & orf19.7310 & & 0.3 & 0.3 & 2.5 & 2.6 & 1.1 & 0.5 & 9.5 & 8.4 & 4.2 & 1.6 \\
\hline & orf19.927 & & 0.4 & 0.2 & 1.3 & 1.8 & 1.0 & 1.6 & 3.1 & 8.4 & 2.3 & 7.1 \\
\hline
\end{tabular}




\section{Table B-2. (Continued)}

\begin{tabular}{|c|c|c|c|c|c|c|c|c|c|c|c|c|}
\hline \multirow[b]{3}{*}{ Process $^{a}$} & \multirow[b]{3}{*}{$\begin{array}{c}\text { orf19 } \\
\text { Designation }\end{array}$} & \multirow[b]{3}{*}{$\begin{array}{c}\text { CGD } \\
\text { Name }\end{array}$} & \multicolumn{6}{|c|}{ Change in Fold Expression } & \multirow{2}{*}{\multicolumn{2}{|c|}{$\begin{array}{c}C A S 5 \text { Ratio } \\
\text { (B/A) }\end{array}$}} & \multirow{2}{*}{\multicolumn{2}{|c|}{$\begin{array}{c}\text { UPC2 Ratio } \\
\text { (C/A) }\end{array}$}} \\
\hline & & & \multicolumn{2}{|c|}{$\begin{array}{c}\text { SC5314+FLC/ } \\
\text { SC5314 (A) } \\
\end{array}$} & \multicolumn{2}{|c|}{$\begin{array}{c}\operatorname{cas} 5 \Delta / \Delta+\mathrm{FLC} / \\
\operatorname{cas} 5 \Delta / \Delta(\mathrm{B}) \\
\end{array}$} & \multicolumn{2}{|c|}{$\begin{array}{c}u p c 2 \Delta / \Delta+\mathrm{FLC} / \\
u p c 2 \Delta / \Delta(\mathrm{C})\end{array}$} & & & & \\
\hline & & & Exp1 & Exp2 & Exp1 & Exp2 & Exp1 & $\operatorname{Exp2}$ & Exp1 & Exp2 & Exp1 & Exp2 \\
\hline \multirow{5}{*}{$\begin{array}{c}\text { Carbohydrate } \\
\text { metabolic process }\end{array}$} & orf19.4270 & MNN13 & 0.5 & 0.5 & 1.4 & 1.2 & 1.1 & 0.9 & 2.6 & 2.3 & 2.0 & 1.7 \\
\hline & orf19.5612 & BMT4 & 0.4 & 0.3 & 0.7 & 0.6 & 1.0 & 0.6 & 1.7 & 2.1 & 2.5 & 2.1 \\
\hline & orf19.6400 & & 0.5 & 0.5 & 1.2 & 1.5 & 1.3 & 1.2 & 2.4 & 3.0 & 2.5 & 2.3 \\
\hline & orf19.6416 & & 0.4 & 0.4 & 2.2 & 3.3 & 3.8 & 2.0 & 5.0 & 7.4 & 8.5 & 4.4 \\
\hline & orf19.6692 & $M N N 26$ & 0.5 & 0.4 & 0.9 & 1.1 & 0.9 & 0.6 & 1.8 & 3.1 & 1.8 & 1.7 \\
\hline \multirow[t]{13}{*}{ Other } & orf19.1159 & & 0.3 & 0.5 & 1.6 & 2.9 & 4.0 & 1.1 & 5.4 & 5.9 & 13.2 & 2.1 \\
\hline & orf19.1697 & & 0.3 & 0.4 & 1.0 & 1.9 & 0.5 & 0.9 & 2.9 & 5.0 & 1.5 & 2.3 \\
\hline & orf19.1757 & & 0.4 & 0.2 & 1.6 & 3.3 & 0.7 & 0.6 & 4.1 & 14.1 & 1.9 & 2.7 \\
\hline & orf19.2018 & & 0.3 & 0.3 & 2.1 & 4.1 & 2.0 & 2.3 & 7.2 & 12.4 & 6.9 & 6.9 \\
\hline & orf19.4774 & $A O X 1$ & 0.5 & 0.4 & 1.3 & 1.2 & 1.6 & 1.1 & 2.7 & 3.0 & 3.3 & 2.7 \\
\hline & orf19.5369 & & 0.4 & 0.4 & 0.7 & 0.8 & 0.7 & 0.7 & 1.8 & 1.9 & 1.9 & 1.7 \\
\hline & orf19.5519 & $G C V 1$ & 0.3 & 0.2 & 1.0 & 1.0 & 1.1 & 0.6 & 2.9 & 5.7 & 3.2 & 3.2 \\
\hline & orf19.568 & SPE2 & 0.4 & 0.2 & 1.4 & 2.0 & 2.1 & 1.4 & 3.8 & 9.3 & 5.5 & 6.8 \\
\hline & orf19.5736 & ALS5 & 0.4 & 0.3 & 0.7 & 1.1 & 3.0 & 2.2 & 1.5 & 3.2 & 6.8 & 6.3 \\
\hline & orf19.5789 & $A D E 8$ & 0.4 & 0.2 & 1.3 & 1.9 & 0.6 & 0.6 & 3.3 & 7.6 & 1.6 & 2.5 \\
\hline & orf19.6146 & $C L G 1$ & 0.4 & 0.5 & 0.6 & 1.0 & 1.5 & 1.0 & 1.6 & 2.3 & 4.0 & 2.2 \\
\hline & orf19.7375 & & 0.5 & 0.3 & 1.3 & 1.8 & 1.1 & 2.1 & 2.7 & 6.7 & 2.2 & 7.5 \\
\hline & orf19.756 & $S A P 7$ & 0.4 & 0.4 & 0.9 & 0.6 & 13.6 & 6.6 & 2.2 & 1.5 & 31.7 & 15.7 \\
\hline \multirow[t]{6}{*}{ Unknown } & orf19.1075 & & 0.3 & 0.5 & 1.5 & 1.4 & 2.5 & 1.4 & 5.1 & 2.7 & 8.9 & 2.8 \\
\hline & orf19.1258 & & 0.4 & 0.2 & 0.9 & 1.2 & 1.0 & 0.4 & 2.5 & 5.2 & 2.7 & 1.6 \\
\hline & orf19.1510 & SRD1 & 0.3 & 0.4 & 0.8 & 1.4 & 1.8 & 0.8 & 2.7 & 3.6 & 6.1 & 2.0 \\
\hline & orf19.1681 & & 0.5 & 0.5 & 0.9 & 0.8 & 3.0 & 3.6 & 1.7 & 1.7 & 5.5 & 7.2 \\
\hline & orf19.2246 & & 0.3 & 0.1 & 0.6 & 0.8 & 0.7 & 2.1 & 2.2 & 5.7 & 2.6 & 14.1 \\
\hline & orf19.2461 & PRN4 & 0.5 & 0.3 & 1.4 & 1.2 & 1.6 & 2.0 & 2.7 & 4.5 & 3.1 & 7.6 \\
\hline
\end{tabular}


Table B-2. (Continued)

\begin{tabular}{|c|c|c|c|c|c|c|c|c|c|c|c|c|}
\hline \multirow[b]{3}{*}{$\operatorname{Process}^{\mathrm{a}}$} & \multirow{3}{*}{$\begin{array}{c}\text { orf19 } \\
\text { Designation }\end{array}$} & \multirow{3}{*}{$\begin{array}{c}\text { CGD } \\
\text { Name } \\
\end{array}$} & \multicolumn{6}{|c|}{ Change in Fold Expression } & & & & \\
\hline & & & \multicolumn{2}{|c|}{$\begin{array}{c}\text { SC5314+FLC/ } \\
\text { SC5314 (A) }\end{array}$} & \multicolumn{2}{|c|}{$\begin{array}{c}\operatorname{cas} 5 \Delta / \Delta+\mathrm{FLC} / \\
\operatorname{cas} 5 \Delta / \Delta(\mathrm{B}) \\
\end{array}$} & \multicolumn{2}{|c|}{$\begin{array}{c}u p c 2 \Delta / \Delta+F L C / \\
u p c 2 \Delta / \Delta(C)\end{array}$} & \multicolumn{2}{|c|}{$\begin{array}{c}\text { CAS5 Ratio } \\
\text { (B/A) }\end{array}$} & \multicolumn{2}{|c|}{$\begin{array}{c}\text { UPC2 Ratio } \\
\text { (C/A) }\end{array}$} \\
\hline & & & Exp1 & Exp2 & Exp1 & Exp2 & Exp1 & Exp2 & Exp1 & Exp2 & Exp1 & Exp2 \\
\hline & orf19.2547 & & 0.5 & 0.2 & 1.7 & 2.7 & 0.9 & 1.0 & 3.5 & 12.1 & 1.9 & 4.7 \\
\hline & orf19.2870 & & 0.5 & 0.4 & 4.1 & 4.6 & 1.5 & 1.1 & 8.0 & 11.2 & 3.0 & 2.8 \\
\hline & orf19.2936 & & 0.4 & 0.3 & 1.2 & 1.6 & 1.9 & 0.7 & 2.8 & 4.9 & 4.6 & 2.2 \\
\hline & orf19.3042 & & 0.5 & 0.4 & 1.0 & 3.3 & 1.1 & 3.5 & 2.1 & 7.6 & 2.4 & 8.0 \\
\hline & orf19.306 & USO5 & 0.5 & 0.5 & 1.8 & 2.0 & 1.3 & 1.7 & 3.4 & 4.1 & 2.4 & 3.5 \\
\hline & orf19.335 & & 0.4 & 0.3 & 0.6 & 0.6 & 0.7 & 0.8 & 1.7 & 2.3 & 2.1 & 3.1 \\
\hline & orf19.3475 & & 0.1 & 0.1 & 0.4 & 0.4 & 2.0 & 2.1 & 3.0 & 3.9 & 17.2 & 20.3 \\
\hline & orf19.348 & SKN2 & 0.4 & 0.3 & 1.4 & 1.3 & 3.2 & 2.3 & 3.3 & 4.9 & 7.5 & 9.0 \\
\hline & orf19.3916 & & 0.2 & 0.2 & 0.9 & 0.6 & 0.4 & 0.5 & 4.0 & 2.9 & 1.8 & 2.1 \\
\hline & orf19.398 & & 0.4 & 0.4 & 1.3 & 1.2 & 1.2 & 1.3 & 2.9 & 3.3 & 2.6 & 3.7 \\
\hline & orf19.4142 & & 0.3 & 0.3 & 1.5 & 6.2 & 2.7 & 1.1 & 5.6 & 19.6 & 9.7 & 3.6 \\
\hline & orf19.4666 & & 0.4 & 0.5 & 1.0 & 1.8 & 1.1 & 1.1 & 2.5 & 3.5 & 2.7 & 2.1 \\
\hline & orf19.5051 & & 0.4 & 0.2 & 1.3 & 2.3 & 0.6 & 0.9 & 3.6 & 9.9 & 1.7 & 4.0 \\
\hline & orf19.5057 & & 0.4 & 0.3 & 5.8 & 1.9 & 1.8 & 0.7 & 14.2 & 6.0 & 4.5 & 2.4 \\
\hline & orf19.5071 & $N R P 1$ & 0.4 & 0.5 & 0.6 & 1.7 & 1.0 & 0.8 & 1.5 & 3.7 & 2.5 & 1.8 \\
\hline & orf19.5841 & & 0.5 & 0.5 & 0.7 & 0.8 & 0.8 & 1.0 & 1.5 & 1.5 & 1.8 & 1.9 \\
\hline & orf19.660 & & 0.4 & 0.4 & 2.0 & 3.5 & 1.8 & 2.1 & 4.4 & 7.8 & 4.0 & 4.8 \\
\hline & orf19.6635 & & 0.5 & 0.4 & 1.1 & 1.0 & 0.9 & 1.7 & 2.1 & 2.9 & 1.6 & 4.8 \\
\hline & orf19.6650 & & 0.5 & 0.4 & 1.1 & 1.5 & 1.8 & 2.1 & 2.1 & 3.4 & 3.5 & 5.0 \\
\hline & orf19.6679 & & 0.1 & 0.3 & 1.0 & 2.8 & 1.0 & 1.5 & 6.5 & 8.7 & 6.8 & 4.5 \\
\hline & orf19.6737 & & 0.5 & 0.4 & 1.0 & 1.2 & 0.9 & 0.6 & 2.0 & 3.3 & 1.8 & 1.7 \\
\hline & orf19.687 & & 0.4 & 0.2 & 0.8 & 1.3 & 0.7 & 0.5 & 1.8 & 5.4 & 1.7 & 1.9 \\
\hline & orf19.7284 & $A R S 2$ & 0.2 & 0.1 & 1.7 & 1.7 & 0.3 & 0.3 & 8.2 & 14.7 & 1.6 & 2.8 \\
\hline & orf19.7427 & & 0.4 & 0.3 & 0.7 & 1.1 & 1.9 & 1.3 & 1.5 & 3.8 & 4.2 & 4.6 \\
\hline & orf19.7553 & & 0.2 & 0.5 & 3.6 & 3.2 & 0.7 & 1.2 & 15.9 & 6.7 & 3.2 & 2.5 \\
\hline
\end{tabular}

${ }^{a}$ Descriptions are from the Candida Genome Database (http://www.candidagenome.org). 


\section{VITA}

Erin M. Vasicek, daughter of Ronald and Sherrill Vasicek and David and Anna Buckler, was born in Plano, TX. In 1988, she and her family moved to Rochester, New York where she enrolled in the Greece Public School District. After graduating from Greece Athena High School in 2003, she attended the Ohio State University in Columbus, Ohio, earning her Bachelor of Science in Molecular Genetics in 2007. Later that year she then matriculated into the Integrated Program in Biomedical Science at the University of Tennessee Health Science Center in Memphis, Tennessee and expects to complete her Doctor of Philosophy in December 2013. 